\title{
The good, the bad, and the intoxicated
}

Citation for published version (APA):

Mason, N. L. (2021). The good, the bad, and the intoxicated: Neuroadaptations underlying effects of cannabis and psilocybin (ab)use. [Doctoral Thesis, Maastricht University]. Maastricht University. https://doi.org/10.26481/dis.20210608nm

Document status and date:

Published: 01/01/2021

DOI:

10.26481/dis.20210608nm

Document Version:

Publisher's PDF, also known as Version of record

\section{Please check the document version of this publication:}

- A submitted manuscript is the version of the article upon submission and before peer-review. There can be important differences between the submitted version and the official published version of record.

People interested in the research are advised to contact the author for the final version of the publication, or visit the DOI to the publisher's website.

- The final author version and the galley proof are versions of the publication after peer review.

- The final published version features the final layout of the paper including the volume, issue and page numbers.

Link to publication

\footnotetext{
General rights rights.

- You may freely distribute the URL identifying the publication in the public portal. please follow below link for the End User Agreement:

www.umlib.nl/taverne-license

Take down policy

If you believe that this document breaches copyright please contact us at:

repository@maastrichtuniversity.nl

providing details and we will investigate your claim.
}

Copyright and moral rights for the publications made accessible in the public portal are retained by the authors and/or other copyright owners and it is a condition of accessing publications that users recognise and abide by the legal requirements associated with these

- Users may download and print one copy of any publication from the public portal for the purpose of private study or research.

- You may not further distribute the material or use it for any profit-making activity or commercial gain

If the publication is distributed under the terms of Article $25 \mathrm{fa}$ of the Dutch Copyright Act, indicated by the "Taverne" license above, 


\section{THE GOOD, THE BAD, AND THE INTOXICATED}

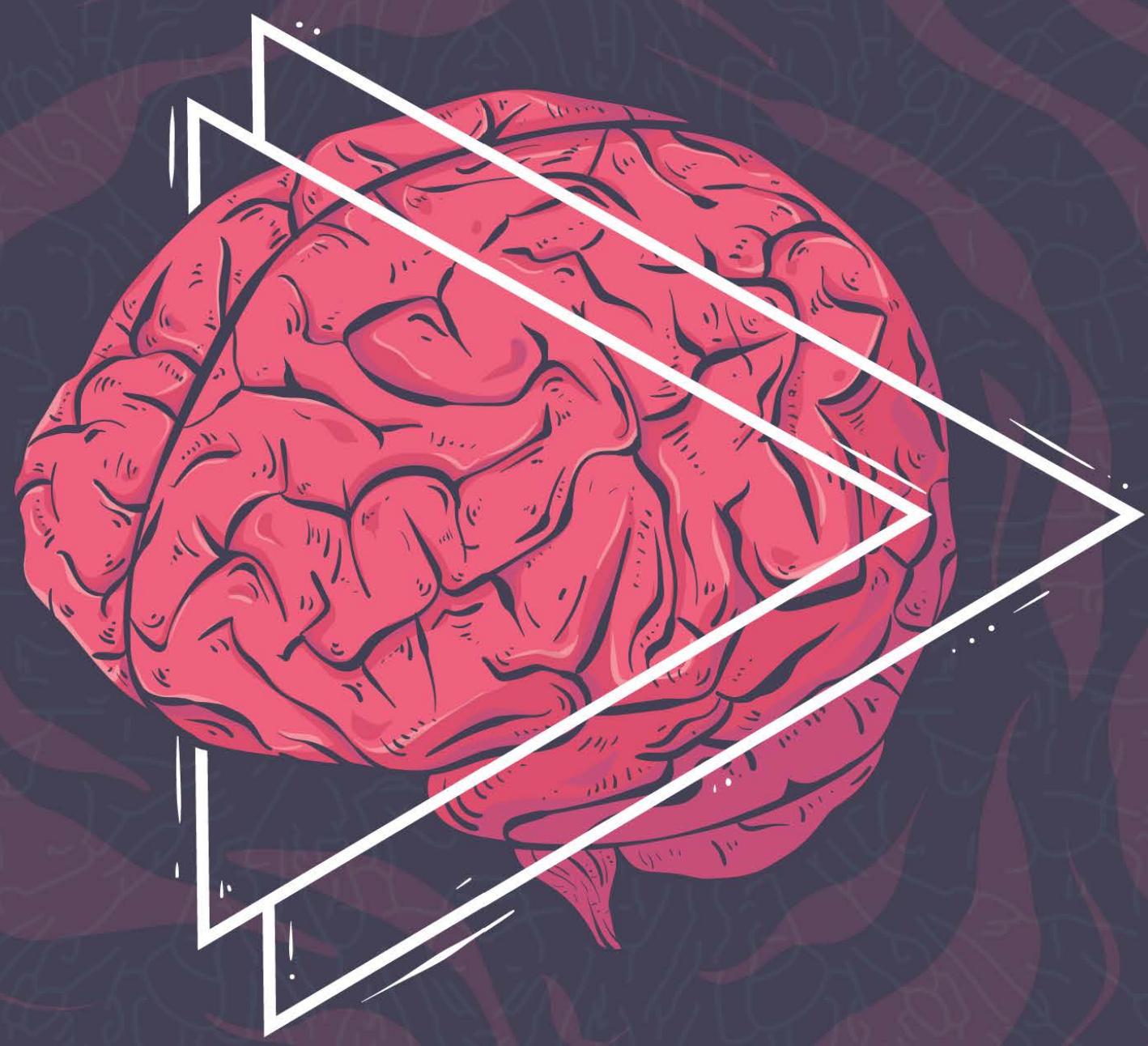

NEUROADAPTATIONS UNDERLYING EFFECTS OF CANNABIS AND PSILOCYBIN (AB) USE 
The good, the bad, and the intoxicated:

Neuroadaptations underlying effects of cannabis and

psilocybin (ab)use

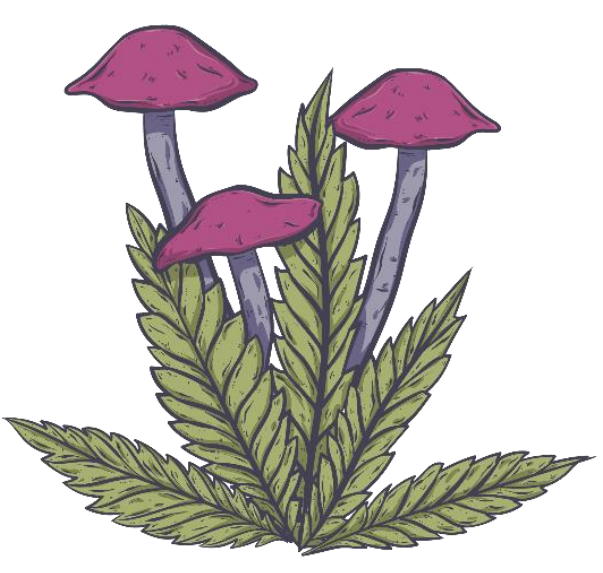

Natasha Leigh Mason 
Cover design: Qubensis

Production: Gildeprint - The Netherlands

ISBN: 978-94-6419-207-0

(C) Natasha L. Mason, 2021 
The good, the bad, and the intoxicated: Neuroadaptations underlying effects of cannabis and psilocybin (ab)use

\section{DISSERTATION}

To obtain the degree of doctor at Maastricht University, on the authority of Rector Magnificus, Prof dr. Rianne M. Letschert, in accordance with the decision of the Board of Deans, to be defended in public on Tuesday, June $8^{\text {th }} 2021$ at 16:00 CET

By

Natasha Leigh Mason 


\section{Supervisors}

Prof. Dr. J.G. Ramaekers

Dr. K.P.C. Kuypers

\section{Co-supervisors}

Dr. E.L. Theunissen

Dr. P. Stiers

\section{Assessment committee}

Prof. Dr. A. Blokland (Chair)

Prof. Dr. D.B. de Araujo

Dr. K.H. Preller

Prof. Dr. W.J. Riedel

Prof. Dr. T.A.M.J. van Amelsvoort 


\section{Contents}

CHAPTER 1 GENERAL INTRODUCTION

CHAPTER 2 CANNABIS INDUCED INCREASE IN STRIATAL GLUTAMATE ASSOCIATED WITH LOSS OF FUNCTIONAL CORTICOSTRIATAL CONNECTIVITY 15

CHAPTER 3 REDUCED RESPONSIVENESS OF THE REWARD SYSTEM IS ASSOCIATED WITH TOLERANCE TO CANNABIS IMPAIRMENT IN CHRONIC USERS

CHAPTER 4 ME, MYSELF, BYE: REGIONAL ALTERATIONS IN GLUTAMATE AND THE EXPERIENCE OF EGO DISSOLUTION WITH PSILOCYBIN 77

CHAPTER 5 SUB-ACUTE EFFECTS OF PSILOCYBIN ON EMPATHY, CREATIVE THINKING, AND SUBJECTIVE WELL-BEING

CHAPTER 6 BRAINWAVES! SPONTANEOUS AND DELIBERATE CREATIVE COGNITION DURING AND AFTER PSILOCYBIN EXPOSURE

CHAPTER 7 DISCUSSION 165

SUMMARY 181

SAMENVATTING 185

IMPACT PARAGRAPH 189

ACKNOWLEDGEMENTS 193

ABOUT THE AUTHOR 199

OUTPUT 201 


\section{Chapter 1}

\section{General Introduction}

Humans have been looking for ways to alter their consciousness by taking substances that are found in nature since time immemorial ${ }^{1}$. Substances like cannabis and psychedelics are used by many people for a myriad of recreational and therapeutic reasons. Namely, individuals report using cannabis for its euphoric and relaxing effects ${ }^{2,3}$, whereas individuals report using a range of psychedelic substances, such as psilocybin, to facilitate self-exploration, enhance creativity, and increase general well-being ${ }^{3-6}$. Importantly, as both substances are currently being investigated for a wide range of clinical implications ${ }^{7,8}$, both recreational and therapeutic use of cannabis and psilocybin will grow substantially. Thus a pertinent question is how these substances work to elicit such effects, and what the long-term consequences of use are. The latter is of particular importance, as single doses of these substances have been suggested to produce adaptations in the brain (neuroadaptations) that underlie acute and long-term behavioral changes. Such behavioral consequences can be seen as maladaptive, like increased tolerance to the rewarding effects of the drug, resulting in the user needing to increase the dose and frequency of use; or adaptive, with users experiencing sustained increases in well-being, long after acute drug effects have worn off. Although research has been investigating the long-term behavioral consequences of cannabis and psilocybin use ${ }^{9,10}$, the underlying neuroadaptations giving rise to such effects, have not been systematically assessed in humans.

\section{Acute administration of cannabis and psilocybin alters neuronal activity levels.}

When a psychoactive substance enters circulation, it crosses the blood-brain barrier, entering the brain and interacting with different chemical pathways. By mimicking natural chemical messengers, the substance binds to receptors in the brain, altering their function and eliciting a drug-distinct combination of behavioral and physiological effects ${ }^{11}$. Acutely, behavioral effects are induced by relatively short-lived changes in brain chemistry and physiology, mainly based on the molecular pharmacology of the specific drug itself ${ }^{12}$. Namely, a person's experiences when using the substance reflects the functional roles of the particular neurotransmitter(s) it disrupts, and where its receptor is distributed among the brain's various functional areas.

Traditionally, research into the acute effects of cannabis and psilocybin has focused on the role of neuromodulatory neurotransmitters in this. In the case of cannabis, the rewarding and behavioral effects produced by $\Delta$-9-tetrahydrocannabinol (THC), its main psychoactive component, have primarily been attributed to increases in dopaminergic neurotransmission throughout fronto-subcortical brain circuits ${ }^{13}$. Whereas the suggested primary mechanism of 
action for the hallucinogenic effects of psilocybin is attributed to the stimulation of serotonin (5HT) $2 \mathrm{~A}$ receptors distributed throughout higher-order cortical areas ${ }^{14}$. That said, as neuromodulators, both dopamine and serotonin induce such a wide range of effects by altering the strength of signal transmissions in neural circuits ${ }^{15-18}$ via regulating drivers of neural activity, such as the excitatory neurotransmitter, glutamate. Accordingly, preclinical studies have indicated a significant role of glutamate in cannabis- and psychedelic-induced effects on brain and behavior ${ }^{14,19-21}$.

Glutamate is the most abundant neurotransmitter in the brain, accounting for $70 \%$ of synaptic neurotransmission ${ }^{22}$. As the primary excitatory neurotransmitter, glutamate release increases the likelihood that the neuron it acts upon will transmit a signal, allowing messages to be spread throughout neural circuits. Through activation of a range of receptors (including AMPA, NMDA, and metabotropic glutamate receptors), the glutamatergic system is essential for fast signaling and information processing in neuronal networks, making glutamate a principal mediator of a myriad of processes, including cognition, emotion, sensory processing, and motor coordination. Thus due to the modulatory influence of serotonin and dopamine on glutamate and the ubiquitous nature of glutamate in driving normal brain function and behavior, its involvement in the acute effects of cannabis and psilocybin on neural activity and associated behavioral outcomes can be expected.

\section{Acute administration can have lasting consequences on brain structure and function}

As well as mediating acute and temporary modulation of brain function, glutamate release and glutamate-coded synaptic modification underlie long-term plasticity in the nervous system. Neuroplasticity refers to the brain's ability to modify, change, and adapt both structure and function in response to intrinsic or extrinsic stimuli23. Structural plasticity includes morphological changes such as alterations in the number of dendrites and spines and the creation of new neurons (neurogenesis) or selective disposal of old neurons (apoptosis). Functional plasticity includes changes in neurotransmitter release and synaptic strength, examples being long-term potentiation (LTP) and long-term depression (LTD), which are considered the neurophysiological substrates of learning and memory ${ }^{24}$. Here, synapses respond to patterns of activity by either strengthening, in response to increased activity, or weakening, in response to decreased activity. Together, these activity-dependent alterations in the number and strength of synaptic connections allow the properties of neuronal circuits to be refined by experience. In the case of adaptation due to a drug experience, they are commonly referred to as drug-induced neuroadaptations ${ }^{25}$. Importantly, evidence from preclinical studies suggests that stimulation of glutamate during the cannabis- and psilocybin-intoxicated state can induce neuroadaptations that 
facilitate enduring functional and behavioral changes that persist even after the substance has been eliminated from the system ${ }^{20,21}$.

\section{Neuroadaptations can be both adaptive and maladaptive.}

Although neuroplasticity is a ubiquitous phenomenon in that our brains are continually changing, a pertinent question is how functionally relevant such alterations are, i.e., what type of behavioral changes do these brain adaptations give rise to. Importantly, behavioral consequences can be either beneficial (mediated by "adaptive" plasticity) or detrimental, causing unwanted symptoms or behavioral patterns (mediated by "maladaptive" plasticity) ${ }^{26}$.

Adaptive drug use

As previously mentioned, individuals report using psychedelics to facilitate self-exploration, broaden consciousness, enhance creativity, and increase general well-being ${ }^{3-6}$. Additionally, recent clinical studies support anecdotal claims that these substances can induce long-term enhancements in feelings of subjective well-being ${ }^{27-32}$ and can provide persisting therapeutic relief for those suffering from anxiety, depression, and addiction27,30,33-36, after just one or two administrations of the drug. Preclinical studies have suggested that these long-term behavioral changes are due to acute increases in extracellular glutamate, resulting in downstream enhancements of brain-derived neurotrophic factor (BDNF), which supports the survival and development of neurons and neurogenesis $20,37-39$.

\section{Maladaptive drug use}

Individuals report using cannabis for its euphoric and relaxing effects ${ }^{3}$. Notably, several studies have suggested that tolerance may develop to the subjective and behavioral effects of cannabis, in that users who frequently use the drug report lower levels of euphoria and cognitive impairment compared to occasional users $9{ }^{90,41}$. Although reduced cognitive impairment could be seen as adaptive, the blunting of the drug's rewarding effects can be seen as unwanted for users, as the high that they search for becomes harder to attain, requiring them to use more of the substance. It has been suggested that tolerance is a form of glutamate-induced neuroplasticity ${ }^{42}$. Accordingly, preclinical studies and (limited) research in chronic cannabis users suggests that repeated exposure to cannabis alters levels of glutamate and glutamate-derived metabolites in brain areas implicated in the rewarding and impairing effects of the drug ${ }^{21}$; potentially mitigating the impact of acute cannabis intoxication on neural activity and associated behavioral outcomes.

\section{Aims of this dissertation}

Although glutamate is implicated in both short- and long-term activity-dependent alterations in the function and formation of neurons, which may mediate acute and persisting behavioral 
effects, the role of glutamate in the psychedelic-(adaptive) and cannabis-induced (maladaptive) consequences in humans has been widely overlooked in research. This represents a large knowledge gap, as understanding the underlying mechanisms of such changes and how they can be influenced is pivotal in understanding both the therapeutic effects of the drugs and long-term consequences of use.

Several experimental studies were conducted to gain more insight into acute neuroadaptations induced by single and repeated drug exposure, which may underlie previously established behavioral effects of cannabis and psilocybin. Although it is methodologically impossible to assess neuronal function and formation in vivo in humans directly, magnetic resonance spectroscopy (MRS) is a non-invasive imaging technique that allows quantification of brain markers that reflect neuroadaptations (i.e., glutamate levels). Additionally, complementary insight can also be obtained by considering alterations in circuit-level interactions between brain regions ${ }^{43,44}$ via resting-state network functional connectivity. Thus utilizing this multimodal methodology, the main goals of the present dissertation were twofold. First, it was assessed whether cannabis and psilocybin acutely alter brain markers that reflect neuroadaptations (i.e., increased glutamate levels), in key brain areas implicated in the action of the drug. Then, it was investigated whether such an increase in glutamate predicts established acute and persisting drugspecific effects.

Regarding cannabis, the focus was on domains that have been found to be blunted after repeated use of the drug (i.e., demonstrating tolerance). These include the rewarding and cognition impairing effects of THC and the effect on dopaminergic neurotransmission. Regarding psilocybin, the focus was on established acute effects implicated in long-term subjective and behavioral changes (e.g., ego dissolution and disrupted resting-state functional connectivity). Additionally, as multiple reports have suggested increased creativity after psychedelic use, the (sub)acute effects and potential underlying mechanisms of psilocybin on creative thinking were assessed.

\section{Outline of the dissertation}

In Chapter 2, the potential of cannabis to acutely alter brain markers that reflect neuroadaptations was assessed. Utilizing a multimodal imaging study e.g., employing both MRS and fMRI, different doses of cannabis were given to occasional users to investigate whether, and at what dose, cannabis increased glutamate levels. It was further assessed whether this glutamate change predicted previously established drug effects, including increases in subjective high, decreases in sustained attention, and alterations in striatal functional connectivity that have been associated with increased dopamine release during exposure to cannabis, cocaine, and methylphenidate. In light of findings from Chapter 2, Chapter 3 investigated the consequence of repeated drug use by 
comparing the influence of cannabis on glutamate levels in occasional vs. chronic users. To investigate the role of glutamatergic neuroadaptations in the experience of tolerance, the relationship between changes in glutamate levels and variables found to be affected by tolerance (subjective high, sustained attention performance, and dopaminergic neurotransmission) was assessed. In Chapter 4, the potential of psilocybin to acutely alter brain markers that reflect neuroadaptations was assessed. Utilizing a multimodal brain imaging approach, the effect of psilocybin was investigated on glutamate levels in key brain regions implicated in the psychedelic experience. Furthermore, the association between glutamate levels and key features of the psychedelic state, e.g., the experience of ego dissolution and disrupted resting-state connectivity, was assessed. The latter were of particular interest, as they have been found to correlate with persisting changes in behavior in previous studies. In Chapter 5, utilizing a naturalistic study design, it was assessed whether psilocybin induced changes in creativity and empathy that outlasted the acute state, as has been reported anecdotally. In view of findings from Chapter 5, Chapter 6 extended the investigation utilizing an experimental design in order to assess whether psilocybin increased creative thinking relatively to placebo and whether acute changes in glutamate and resting-state network connectivity predicted performance on the measures of creativity acutely and after one week. 


\section{Chapter 1}

\section{References}

1 Merlin, M. D. Archaeological evidence for the tradition of psychoactive plant use In the old world. Economic Botany 57, 295-323, doi:10.1663/0013-0001(2003)057[0295:AEFTTO]2.0.CO;2 (2003).

2 Osborne, G. B. \& Fogel, C. Understanding the motivations for recreational marijuana use among adult Canadians. Substance use \& misuse 43, 539-572; discussion 573-539, 585-537, doi:10.1080/10826080701884911 (2008).

3 Kettner, H., Mason, N. L. \& Kuypers, K. P. C. Motives for classical and novel psychoactive substances use of psychedelic polydrug-users Contemporary Drug Problems (2019). Mason, N. L. \& Kuypers, K. P. Mental health of a self-selected sample of psychedelic users and self-medication practices with psychedelics. Journal of Psychedelic Studies 2, 45-52 (2018).

5 Hutten, N. R. P. W., Mason, N. L., Dolder, P. C. \& Kuypers, K. P. C. Motives and Side-Effects of Microdosing With Psychedelics Among Users. The international journal of neuropsychopharmacology 22, 426-434, doi:10.1093/ijnp/pyz029 (2019).

Baggott, M. J. Psychedelics and creativity: a review of the quantitative literature. PeerJ PrePrints 3, e1202v1201, doi:10.7287/peerj.preprints.1202v1 (2015).

$7 \quad$ Nutt, D., Erritzoe, D. \& Carhart-Harris, R. Psychedelic Psychiatry's Brave New World. Cell 181, 24-28 (2020).

8 Montero-Oleas, N., Arevalo-Rodriguez, I., Nuñez-González, S., Viteri-García, A. \& Simancas-Racines, D. Therapeutic use of cannabis and cannabinoids: an evidence mapping and appraisal of systematic reviews. BMC complementary medicine and therapies 20, 12 (2020).

9 Colizzi, M. \& Bhattacharyya, S. Cannabis use and the development of tolerance: a systematic review of human evidence. Neurosci Biobehav Rev 93, 1-25, doi:10.1016/j.neubiorev.2018.07.014 (2018).

Aday, J. S., Mitzkovitz, C. M., Bloesch, E. K., Davoli, C. C. \& Davis, A. K. Long-term effects of psychedelic drugs: A systematic review. Neuroscience \& Biobehavioral Reviews 113, 179-189, doi:https://doi.org/10.1016/j.neubiorev.2020.03.017 (2020).

Kalivas, P. W. \& O'Brien, C. Drug Addiction as a Pathology of Staged Neuroplasticity. Neuropsychopharmacology 33, 166180, doi:10.1038/sj.npp.1301564 (2008).

Bloomfield, M. A., Ashok, A. H., Volkow, N. D. \& Howes, O. D. The effects of Delta9-tetrahydrocannabinol on the dopamine system. Nature 539, 369-377, doi:10.1038/nature20153 (2016).

Nichols, D. E. Psychedelics. Pharmacological reviews 68, 264-355, doi:10.1124/pr.115.011478 (2016).

Gardoni, F. \& Bellone, C. Modulation of the glutamatergic transmission by Dopamine: a focus on Parkinson, Huntington and Addiction diseases. Frontiers in cellular neuroscience 9, 25, doi:10.3389/fncel.2015.00025 (2015). Ciranna, L. Serotonin as a modulator of glutamate- and GABA-mediated neurotransmission: implications in physiological functions and in pathology. Curr Neuropharmacol 4, 101-114, doi:10.2174/157015906776359540 (2006).

Pehrson, A. L. \& Sanchez, C. Serotonergic modulation of glutamate neurotransmission as a strategy for treating depression and cognitive dysfunction. CNS spectrums 19, 121-133, doi:10.1017/s1092852913000540 (2014).

Boto, T. \& Tomchik, S. M. The Excitatory, the Inhibitory, and the Modulatory: Mapping Chemical Neurotransmission in the Brain. Neuron 101, 763-765 (2019).

Vollenweider, F. X. \& Kometer, M. The neurobiology of psychedelic drugs: implications for the treatment of mood disorders. Nature reviews. Neuroscience 11, 642-651, doi:10.1038/nrn2884 (2010). Vollenweider, F. X. \& Preller, K. H. Psychedelic drugs: neurobiology and potential for treatment of psychiatric disorders. Nature Reviews Neuroscience, doi:10.1038/s41583-020-0367-2 (2020).

Colizzi, M., McGuire, P., Pertwee, R. G. \& Bhattacharyya, S. Effect of cannabis on glutamate signalling in the brain: A systematic review of human and animal evidence. Neuroscience and Biobehavioral Reviews 64, 359-381, doi:10.1016/j.neubiorev.2016.03.010 (2016). doi:10.1016/0301-0082(90)90013-7 (1990). Cramer, S. C. et al. Harnessing neuroplasticity for clinical applications. Brain : a journal of neurology 134, 1591-1609, doi:10.1093/brain/awr039 (2011). Bliss, T. V. P. \& Collingridge, G. L. A synaptic model of memory: long-term potentiation in the hippocampus. Nature 361, 31-39, doi:10.1038/361031a0 (1993). (2015). 3-12, doi:10.1016/B978-0-444-52901-5.00001-0 (2013).

Carhart-Harris, R. L. et al. Psilocybin with psychological support for treatment-resistant depression: an open-label feasibility study. The Lancet Psychiatry 3, 619-627 (2016). 
Griffiths, R. R., Richards, W. A., McCann, U. \& Jesse, R. Psilocybin can occasion mystical-type experiences having substantial and sustained personal meaning and spiritual significance. Psychopharmacology (Berl) 187, 268-283; discussion 284-292, doi:10.1007/s00213-006-0457-5 (2006).

Griffiths, R. R., Richards, W. A., Johnson, M. W., McCann, U. D. \& Jesse, R. Mystical-type experiences occasioned by psilocybin mediate the attribution of personal meaning and spiritual significance 14 months later. Journal of psychopharmacology 22, 621-632 (2008). Johnson, M. W., Garcia-Romeu, A. \& Griffiths, R. R. Long-term follow-up of psilocybin-facilitated smoking cessation. The American journal of drug and alcohol abuse 43, 55-60, doi:10.3109/00952990.2016.1170135 (2017). Uthaug, M. V. et al. Sub-acute and long-term effects of ayahuasca on affect and cognitive thinking style and their association with ego dissolution. Psychopharmacology, 1-11 (2018). Griffiths, R. R. et al. Psilocybin produces substantial and sustained decreases in depression and anxiety in patients with life-threatening cancer: A randomized double-blind trial. Journal of Psychopharmacology 30, 1181-1197, doi:10.1177/0269881116675513 (2016). Gasser, P. et al. Safety and efficacy of lysergic acid diethylamide-assisted psychotherapy for anxiety associated with lifethreatening diseases. The Journal of nervous and mental disease 202, 513-520, doi:10.1097/NMD.0000000000000113 (2014).

Palhano-Fontes, F. et al. Rapid antidepressant effects of the psychedelic ayahuasca in treatment-resistant depression: a randomized placebo-controlled trial. Psychological medicine 49, 655-663, doi:10.1017/s0033291718001356 (2019). Grob, C. S. et al. Pilot study of psilocybin treatment for anxiety in patients with advanced-stage cancer. Archives of general psychiatry 68, 71-78, doi:10.1001/archgenpsychiatry.2010.116 (2011). Sanches, R. F. et al. Antidepressant Effects of a Single Dose of Ayahuasca in Patients With Recurrent Depression: A SPECT Study. Journal of clinical psychopharmacology 36, 77-81, doi:10.1097/jcp.0000000000000436 (2016). Vollenweider, F. X. \& Kometer, M. The neurobiology of psychedelic drugs: Implications for the treatment of mood disorders. Nature Reviews Neuroscience 11, 642-651, doi:http://dx.doi.org/10.1038/nrn2884 (2010).

Vaidya, V. A., Marek, G. J., Aghajanian, G. K. \& Duman, R. S. 5-HT2A receptor-mediated regulation of brain-derived neurotrophic factor mRNA in the hippocampus and the neocortex. The Journal of neuroscience : the official journal of the Society for Neuroscience 17, 2785-2795 (1997). Cavus, I. \& Duman, R. S. Influence of estradiol, stress, and 5-HT2A agonist treatment on brain-derived neurotrophic factor expression in female rats. Biological psychiatry 54, 59-69 (2003). Ramaekers, J. G., Kauert, G., Theunissen, E., Toennes, S. W. \& Moeller, M. Neurocognitive performance during acute THC intoxication in heavy and occasional cannabis users. Journal of psychopharmacology 23, 266-277 (2009). Ramaekers, J. G. et al. Tolerance and cross-tolerance to neurocognitive effects of THC and alcohol in heavy cannabis users. Psychopharmacology (Berl) 214, 391-401 (2011). doi:10.1016/j.bcp.2007.06.039 (2008). Koob, G. F. \& Volkow, N. D. Neurocircuitry of addiction. Neuropsychopharmacology 35, 217-238, doi:10.1038/npp.2009.110 (2010).

Sutherland, M. T., McHugh, M. J., Pariyadath, V. \& Stein, E. A. Resting state functional connectivity in addiction: Lessons learned and a road ahead. Neurolmage 62, 2281-2295, doi:10.1016/j.neuroimage.2012.01.117 (2012). 


\title{
Chapter 2
}

\section{Cannabis induced increase in striatal glutamate associated with loss of functional corticostriatal connectivity}

\author{
Mason, N.L., Theunissen, E.L., Hutten, N.R.P.W., Tse, D.H.Y., Toennes, Jansen, J.F.A., S.W., Stiers, \\ P., and Ramaekers, J.G.
}

Cannabis is the most commonly used illicit drug and is known to alter state of consciousness and impair neurocognitive function. However, the mechanisms underlying these effects have yet to be fully elucidated. Rodent studies suggest that $\Delta 9$-tetrahydrocannabinol (THC) activates dopaminergic neurons in the limbic system, subsequently enhancing dopamine, which is implicated in the rewarding effects of cannabis. Additional evidence suggests that THC may act indirectly on dopamine firing by modulating GABA and glutamate release. This double-blind, placebo-controlled study assessed the acute influence of two doses of THC on brain kinetics of glutamate, GABA, and dopamine, in relation to behavioral outcomes, by using magnetic resonance spectroscopy and functional magnetic resonance imaging. Twenty occasional cannabis users received acute doses of cannabis ( $300 \mu \mathrm{g} / \mathrm{kg} \mathrm{THC}$ ) and placebo, in one of two dose regimes (full dose and divided dose), during two separate testing days. Administration of THC increased striatal glutamate concentrations, and dopamine as indicated by a reduction in functional connectivity (FC) between the nucleus accumbens (NAc) and cortical areas. Alterations in glutamate and FC were dose dependent and evident in the full dose group where THC serum concentrations exceeded $2 \mathrm{ng} / \mathrm{ml}$ at T-max. Average glutamate changes correlated strongly with FC alterations. Additionally, THC induced changes in FC correlated with feelings of subjective high and decreased performance on an attention task. Taken together, this suggests that THC elicits subjective and cognitive alterations via increased striatal dopaminergic activity and loss of corticostriatal connectivity, which is associated with an increase in striatal glutamate.

European Neuropsychopharmacology, 2019; 29(2), 247-256 


\section{Chapter 2}

\section{Introduction}

Cannabis is the most commonly used illicit drug in the world. About $4 \%$ of the global population have used cannabis and $10 \%$ of those develop daily use patterns ${ }^{1}$. However, with changing legalization and a growing interest in therapeutic utility, there is an increasing trend of use $\mathrm{e}^{2,3}$.

The acute effects of cannabis on subjective and behavioral state are well known. Studies have shown that cannabis induces an increase in feelings of euphoria, altered perception, and relaxation ${ }^{4-6}$, and a dose-related decrease in performance on motor, memory, learning, and attentional tasks ${ }^{7-9}$ when serum delta-9-tetrahydrocannabinol (THC) concentration levels surpass $2 \mathrm{ng} / \mathrm{ml}^{10}$. Nonetheless, the mechanisms underlying these acute effects have yet to be fully elucidated. THC, the main psychoactive component of cannabis, acts on cannabinoid (CB1) receptors distributed in motor and cognitive regions ${ }^{11,12}$. These receptors can be found in moderate to high densities in the cerebellum, hippocampus, frontal cortex, posterior cingulate, and the striatum ${ }^{11,13}$. Previous pharmacological neuroimaging studies investigating acute effects of $\triangle 9$-THC on brain activation have found increased regional cerebral blood flow (rCBF) in frontal, limbic, paralimbic, and cerebellar regions ${ }^{14}$. These alterations have been found to correlate with subjective levels of intoxication ${ }^{15,16}$ and with task performance ${ }^{17,18}$. However, it is likely that these acute functional and behavioral changes induced by cannabis are accompanied by changes in neurochemistry ${ }^{19}$. As the regions of functional change under the influence of cannabis involve those implicated with other substance use disorders, like dopamine reward pathways ${ }^{20}$, it is suggested that alterations in dopaminergic function may be associated with some of the behavioral effects of $\mathrm{THC}^{21}$.

Rodent studies assessing the acute actions of THC suggest that THC leads to a selective increase of activity in dopaminergic neurons in the ventral tegmental area (VTA), dosedependently increasing dopamine baseline neuron firing rates ${ }^{22,23}$. These dopamine neurons in the VTA target both neurons located in the nucleus accumbens (NAc), forming the mesolimbic dopamine pathway, as well as in the frontal cortex ${ }^{24}$. Accordingly, THC has been shown to increase dopamine in striatal areas, the NAc, and pre-frontal cortex ${ }^{25-28}$. Dopaminergic modulation in these brain areas is implicated in the euphoric and rewarding effects of cannabis and other drugs of abuse $^{28,29}$, as well as the disruption on cognitive ${ }^{25}$ and motor ${ }^{30}$ function seen when under the influence of cannabis.

However, it has been suggested that THC may not act directly on dopamine firing, but instead acts indirectly by inhibiting glutamate release onto gamma-aminobutyric acid (GABA) neurons in the VTA and NAc ${ }^{31-33}$. Accordingly, consistent evidence from animal models suggests that THC disrupts glutamate signaling by depressing glutamate synaptic transmission ${ }^{31}$ in a doseand brain region- dependent manner ${ }^{34}$. In humans, only a few studies have investigated the effects 
of cannabis use on glutamate signaling in the brain. These studies have found reduced levels of glutamate-related metabolites in the basal ganglia ${ }^{35,36}$ and anterior cingulate cortex ${ }^{19,37}$ in chronic cannabis users during abstinence. As these brain areas have been implicated in contributing to the behavioral effects of cannabis, these studies suggest that cannabis use affects cortical glutamate levels, and that these neurotransmitter changes mediate the behavioral effects. However, the acute effects of THC on glutamate signaling in the human brain and its interplay with GABA and dopamine transmission have yet to be assessed.

The present study was therefore designed to assess acute influences of two different dose regimens of cannabis on brain kinetics of glutamate, GABA, and dopamine in the limbic system during the absorption and elimination phase of THC. One group ( $N=10)$ received a full dose of 300 $\mu \mathrm{g} / \mathrm{kg}$ THC that was expected to induce subjective high and affect cognitive performance. A second $(\mathrm{N}=10)$ group received 3 successive doses of $100 \mu \mathrm{g} / \mathrm{kg}$, separated in time, that were expected to produce low THC serum concentrations (i.e. around $2 \mathrm{ng} / \mathrm{ml}$ ) with little behavioral interference ${ }^{10}$. Participants in both groups also received placebo during a separate testing day. Ultra-High Field (7T) proton magnetic resonance imaging $\left({ }^{1} \mathrm{H}\right.$ MRS), a non-invasive imaging technique that allows in vivo measurement of neurometabolites and neurotransmitters, was used to assess glutamate and GABA levels in the striatum and anterior cingulate cortex. Resting state functional magnetic resonance imaging ( $\mathrm{fMRI}$ ) was measured to determine functional connectivity between the regions of interest (ROI) in the NAc and remote cortical areas, as an indirect measure of dopaminergic stimulation ${ }^{38,39}$. It was expected that when serum THC concentration levels at Tmax were above $2 \mathrm{ng} / \mathrm{ml}$, cannabis would alter glutamate and GABA levels, and would lead to dopaminergic stimulation, reducing functional corticostriatal connectivity ${ }^{38,39}$. It was further expected that these functional changes would correlate with changes in subjective high and task performance in a dose-dependent manner.

\section{Experimental Procedures}

\section{Participants}

In total, 20 healthy, occasional cannabis users (male $\mathrm{N}=12$, female $\mathrm{N}=8$ ) completed both treatment conditions. On average, participants had been occasional users of cannabis for 4.85 years (min-max: 2-11), using a mean frequency (SE) of 5.33 (.758) times a month. For further details, see Supplement and table S1.

This study was conducted according to the code of ethics on human experimentation

established by the declaration of Helsinki (1964) and amended in Fortaleza (Brazil, October 2013) and in accordance with the Medical Research Involving Human Subjects Act (WMO) and was approved by the Academic Hospital and University's Medical Ethics committee. All participants 
were fully informed of all procedures, possible adverse reactions, legal rights and responsibilities, expected benefits, and their right for voluntary termination without consequences.

\section{Design, doses, and administration}

This study was conducted according to a double-blind, placebo-controlled, mixed cross-over design. Occasional cannabis users were randomized to one of two groups. In the full dose group $(\mathrm{N}=10)$ participants received $300 \mu \mathrm{g} / \mathrm{kg}$ THC (Bedrobinol; $13.5 \% \mathrm{THC}$ ) vapor in one full dose. In the divided dose group ( $\mathrm{N}=10)$ participants received $300 \mu \mathrm{g} / \mathrm{kg}$ THC vapor divided over 3 successive doses of $100 \mu \mathrm{g} / \mathrm{kg}$, separated by 30 minutes. Participants also received the relative placebo $(0 \% \mathrm{THC})$ on a separate day, separated by a minimum wash-out period of 7 days to avoid cross-condition contamination. Treatment orders and doses were randomly assigned to subjects according to a balanced, block design. The dosage of cannabis was tailored to individual participants in order to reach $300 \mu \mathrm{g} / \mathrm{kg}$ bodyweight THC, which has previously been found to be well tolerated by subjects with an average experience of cannabis use ${ }^{40,41}$. For an overview of the testing day schedule, see Table 2 .

\section{Procedures}

Upon arrival, a baseline blood sample was taken and baseline vital signs (blood pressure and heart rate) were measured. After measurements, the participant was placed in the MRI scanner, where after localizer scans and a T1 structural scan were taken, they inhaled the placebo or cannabis vapor. Throughout the intoxication and elimination phase (approximately a 1.5 hour time window) functional connectivity and metabolic changes were assessed three separate times at regular intervals using resting state $\mathrm{fMRI}$ and magnetic resonance spectroscopy (MRS) respectively. Participants were also required to perform an attention task, complete a subjective high questionnaire, and give 5 blood samples at fixed intervals during and after scanning. Participants stayed under supervision until the researcher deemed they were fit to go home. For details, please see Supplement.

\section{Proton Spectra}

Single-voxel proton magnetic resonance spectroscopy (MRS) measurements were performed on a MAGNETOM 7T MR scanner (Siemens Healthineers, Erlangen, Germany) with a whole-body gradient set (SC72; maximum amplitude, $70 \mathrm{mT} / \mathrm{m}$; maximum slew rate, $200 \mathrm{~T} / \mathrm{m} / \mathrm{s}$ ) and using an single-channel transmit/32-channel receive head coil (Nova Medical, Wilmington, MA, USA). Spectroscopic voxels of interest were placed by a trained operator at the Anterior Cingulate Cortex $(A C C)$ (voxel size $=25 \times 20 \times 17 \mathrm{~mm}^{3}$ ) and the right striatum (voxel size $=20 \times 20 \times 20 \mathrm{~mm}^{3}$ ). Spectra were acquired with stimulated echo acquisition mode (STEAM) ${ }^{42}$ sequence using the following parameters: $\mathrm{TE}=6.0 \mathrm{~ms}, \mathrm{TM}=10.0 \mathrm{~ms}, \mathrm{TR}=5.0 \mathrm{~s}, \mathrm{NA}=64$, flip angle $=90^{\circ}, \mathrm{RF}$ bandwidth $=4.69$ 
$\mathrm{kHz}$, RF centred at $2.4 \mathrm{ppm}$, receive bandwidth $=4.0 \mathrm{kHz}$, vector size $=2048,16$-step phase cycling, acquisition time $=5: 20 \mathrm{~min}$. Outcome measures for MRS were concentration ratios of glutamate and GABA to total Creatine (Creatine + Phospho-Creatine). For further information and fit quality measures see Supplement.

\section{Resting state $f M R I$}

During resting state, 258 whole-brain EPI volumes were acquired (TR=1400 ms; TE= 21 ms; field of view $($ FOV $)=198 \mathrm{~mm}$; flip angle $=60^{\circ}$; oblique acquisition orientation; interleaved slice acquisition; 72 slices; slice thickness $=1.5 \mathrm{~mm}$; voxel size $=1.5 \times 1.5 \times 1.5 \mathrm{~mm}$ ). During resting state scans, participants were shown a black cross on a white background, and were instructed to focus on the cross while attempting to clear their mind, and lay as still as possible.

Resting state image preprocessing were conducted using SPM8 (Statistical Parametric Mapping, Wellcome Trust Centre for Neuroimaging, Institute of Neurology, University College London) Preprocessing steps included: motion correction (registered to the first image with second degree B-spline interpolation), coregistration (linking of functional to anatomical scans), and spatial normalization (the mean EPI image of each session was matched to SPM8's EPI template in Montreal Neurological Institute [MNI] space) where after the parameters were applied to all images of that session. During normalization voxel size was $1.5 \times 1.5 \times 1.5 \mathrm{~mm}$. Finally, the data were smoothed at $3 \mathrm{~mm}$ Gaussian kernel.

\section{Functional Connectivity}

Functional connectivity data were produced with the MATLAB toolbox DPARSF ${ }^{43}$. Linear trends of time courses were removed followed by low band-pass filtered (0.01-0.08 HZ) of the preprocessed data to remove 'noise' attributable to physiological parameters. Nuisance covariates (motion paramaeters, white matter signal, CSF signal) were also removed. Two spheres (4 mm radius) were created that were located (in MNI space) in the left $(-9,9,-9)$ and right $(9,9,-9)$ NAc. Seed locations were in agreement with previous work validating structural and functional subdivisions of the $\mathrm{NAc}^{44,45}$. Average time courses were obtained for each sphere separately and correlational analysis was performed voxel wise to generate functional connectivity maps for each sphere. The correlation coefficient map was converted into z maps by Fisher's r-to-z transform to improve normality ${ }^{43,46}$. This is in accordance with previous studies investigating drug induced changes in functional connectivity, utilizing such changes as an indirect measure of dopaminergic stimulation, as dopamine levels cannot be directly assessed by MR methods ${ }^{38,39}$.

\section{Psychomotor Vigilance Task}


The psychomotor vigilance task is a sustained-attention, reaction-time task that measures the speed with which participants respond to a visual stimulus ${ }^{47}$. The participant is instructed to press a button as soon as the stimulus appears (red circle). The outcome measure of this task was number of attentional lapses (reaction time $>500$ ms). For more information, see Supplement.

\section{Subjective High}

Participants rated their subjective high on visual analogue scales $(10 \mathrm{~cm})$ on four consecutive time points after treatment administration. Participants had to indicate how high they felt at that moment, compared with the most high they have ever felt ( $0=$ not high at all; $10=e x t r e m e l y ~ h i g h)$.

\section{Pharmacokinetic Measures}

Blood samples $(8 \mathrm{~mL}$ ) to determine cannabinoid concentrations (THC and metabolites OH-THC and $\mathrm{THC}-\mathrm{COOH}$ ) were taken at baseline, 10, 30, 50, and 70 minutes post administration. Blood samples were centrifuged and serum was frozen at $-20{ }^{\circ} \mathrm{C}$ until analyses for pharmacokinetic assessments. Cannabinoid concentrations were determined using a validated and proficiency test approved forensic routine method consisting of an automated solid-phase extraction and gas chromotrography with tandem mass spectrometric detection with a limit of quantification of $0.3 \mathrm{ng} / \mathrm{ml}$ or less.

\section{Statistical Analysis}

Subjective and cognitive effects. Statistical analysis of subjective high and task performance were conducted in IBM SPSS Statistics 24 using a mixed model analysis consisting of the within-subject factors Treatment (THC and placebo) and Time after smoking (3 levels), and the between-subject factor of Dose (full and divided dose). Due to main effect of Treatment or Treatment x Dose, a second analysis was performed for each dose, with treatment and time as within-subject factors. The alpha criterion level of significance was set at $p=0.05$. Due to a violation of the assumption of normality, the data for the number of lapses were log transformed.

Metabolite concentrations. Due to violations of the assumption of normality, separate Friedman's non-parametric tests were used to assess metabolite concentration per time point for each dose.

fMRI data. Functional connectivity data (i.e. correlation coefficient maps for each individual in each treatment condition at each time point) were analyzed in 2 GLM models in SPM 12. In the first GLM, data entered a full factorial model with treatment (THC and placebo) and time point (3 levels) as within-subject factors and dose (full and divided dose) as a between-subject factor. Due to main effect of dose, a second GLM was performed for each dose, with treatment and time point as within-subject factors. From this model main effects of treatment were identified 
for the full dose group, but not the divided dose group.

Correlation analysis. Voxel wise correlation analysis between FC data and absolute average change scores of striatal glutamate, subjective high, and number of attention lapses were conducted. Individual treatment maps (placebo > THC) were entered into one-sample t-tests in SPM, with the absolute average change scores of striatal glutamate, subjective high, and number of attention lapses. Maps were corrected for multiple comparisons at the cluster level using the family wise error correction (FWE). Average mean voxel activation of SPM identified clusters were put into SPSS and Pearson's correlations were performed to get correlation strengths.

\section{Results}

\section{THC Concentrations in Serum}

Mean (SE) concentrations of $\mathrm{THC}, \mathrm{THCOH}$, and $\mathrm{THCCOH}$ in serum are given in Table 1.

Table 1. Time course for mean (S.E.) concentrations of THC and its metabolites in serum $(\mathrm{ng} / \mathrm{ml}$ ) following smoking two doses of THC (group 1 who received one full dose of $300 \mu \mathrm{g} / \mathrm{kg}$ THC and group 2 who received 3 successive doses of $100 \mu \mathrm{g} / \mathrm{kg}$ ), as assessed by gas chromatography-mass spectrometry (GC-MS)

\begin{tabular}{|c|c|c|c|c|c|c|}
\hline \multirow{3}{*}{$\begin{array}{l}\text { Time } \\
\text { Relative to } \\
\text { Smoking }\end{array}$} & \multicolumn{6}{|c|}{ Serum (GC-MS) } \\
\hline & \multicolumn{3}{|l|}{ Group 1} & \multicolumn{3}{|l|}{ Group 2} \\
\hline & $\mathrm{THC}$ & $\mathrm{THC}-\mathrm{OH}$ & $\mathrm{THC}-\mathrm{COOH}$ & $\mathrm{THC}$ & $\mathrm{THC}-\mathrm{OH}$ & $\mathrm{THC}-\mathrm{COOH}$ \\
\hline 0 & $0.05(.05)$ & $0.03(.03)$ & $0.80(.33)$ & $\begin{array}{l}.019 \\
(0.02)\end{array}$ & $.07(.05)$ & $1.85(.70)$ \\
\hline 6 & $7.03(1.83)$ & $1.97(.43)$ & $9.43(2.30)$ & $1.66(.49)$ & $.63(.18)$ & $3.14(.96)$ \\
\hline 28 & $2.26(.63)$ & $0.91(.29)$ & $7.38(2.00)$ & $2.82(.44)$ & $.97(.10)$ & $4.72(1.06)$ \\
\hline 50 & $1.87(.52)$ & $0.89(.27)$ & $7.52(2.01)$ & $2.95(.62)$ & $.89(.21)$ & $4.86(.10)$ \\
\hline 67 & $2.52(.71)$ & $1.07(.26)$ & $9.64(2.16)$ & $2.18(.35)$ & $.80(.16)$ & $6.82(1.50)$ \\
\hline
\end{tabular}

\section{Subjective and Cognitive Effects}

Mixed model analysis showed a significant main effect of Treatment $[F(1,17)=30.491, P<.0001]$ and Treatment $\times$ Dose $[F(1,17)=9.169, P=.008]$ on subjective high and a main effect of Treatment $[F(1,16)=7.278, P=.016]$ on number of attentional lapses. Further analysis revealed that ratings of subjective high were significantly increased by THC in the full dose group $[F(1,8)=50.330, P=$ $.000]$, but not in the divided dose group $(P>$.1). THC administration also increased the number of 
attentional lapses during the psychomotor vigilance test in the full dose group $[F(1,7)=10.061, P$ $=.016]$, but not in the divided dose group $(P>.1)$. There were no effects of THC on the other outcomes of the psychomotor vigilance test. Mean scores of variables significantly affected by THC are shown in Figure 1.

A.

Full Dose

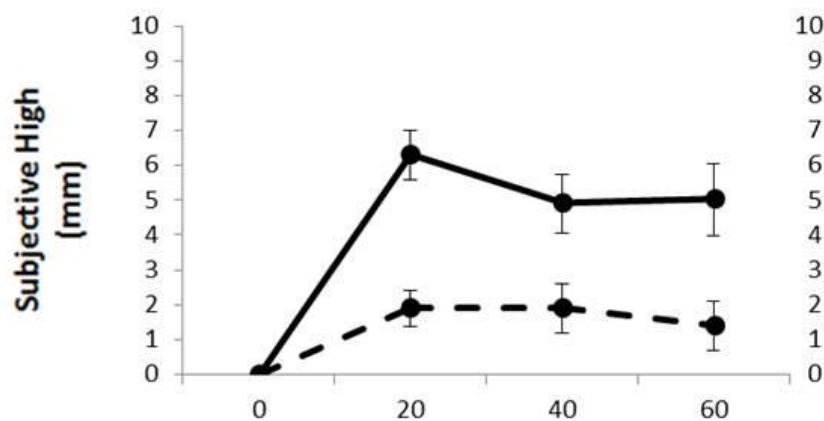

B.

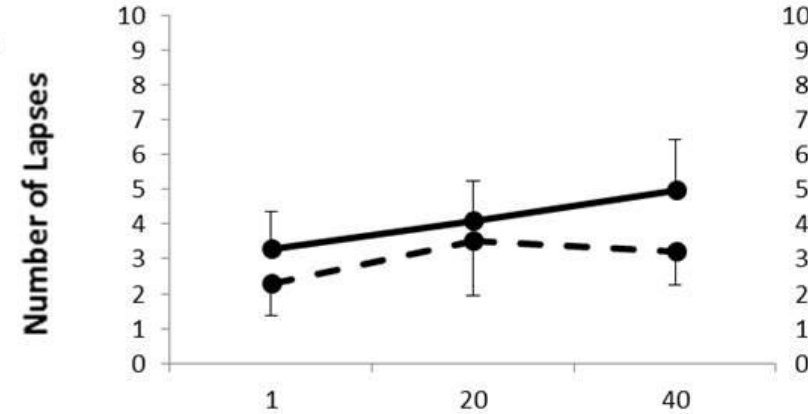

Time relative to smoking (minutes)
Divided Dose
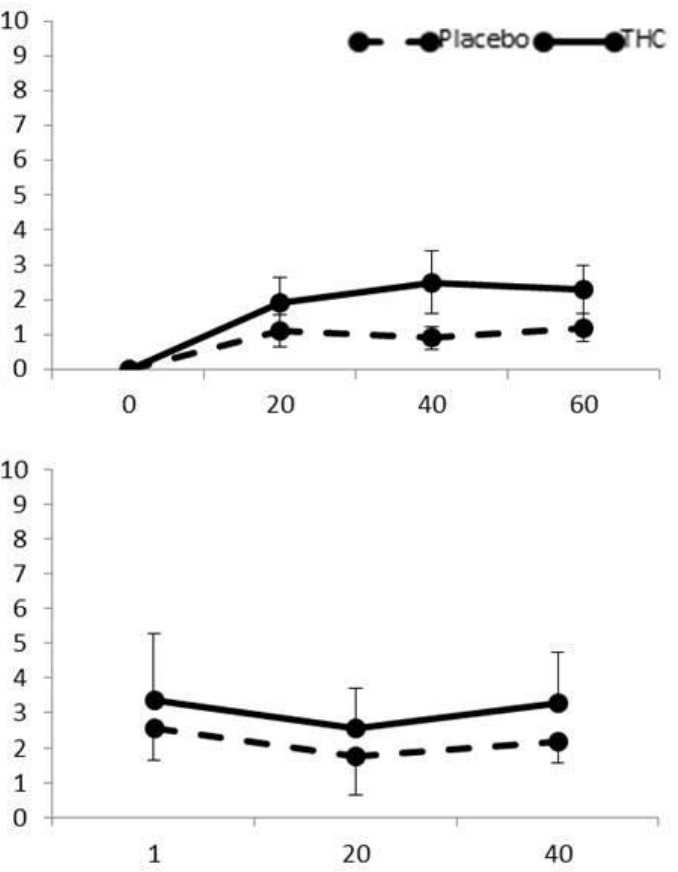

Time relative to smoking (minutes)

Figure 1. Subjective and cognitive effects. A. Group 1 and Group 2 mean (SE) subjective high for both treatments (THC vs placebo) as a function of time relative to smoking (0,20,30, and 60 minutes). B. Group 1 and Group 2 mean (SE) number of lapses (RT>500 ms) for both treatments (THC vs placebo) as a function of time relative to smoking (1, 20, and 40 minutes).

\section{Metabolite Concentrations}

Separate Friedman's non-parametric tests showed that, relative to placebo, THC significantly increased glutamate/PCr $+\mathrm{Cr}$ (Glutamate) concentrations in the right striatum at both 30 minutes $\left[\chi^{2}(1)=9.800, P=.002\right]$ and 50 minutes $\left[\chi^{2}(1)=8.000, P=.005\right]$ post administration. Separate analysis per group showed that this increase was found in the full dose group at both 30 minutes $\left[\chi^{2}(1)=6.400, P=.011\right]$ and 50 minutes $\left[\chi^{2}(1)=5.444, P=.020\right]$, but not the divided dose group (Figure 2). There were no effects of THC on GABA/PCr + $\mathrm{Cr}(\mathrm{GABA})$ in the striatum, or on GABA or 
Glutamate in the ACC, after either dose. Mean scores of metabolites unaffected by THC are shown in Table S3.

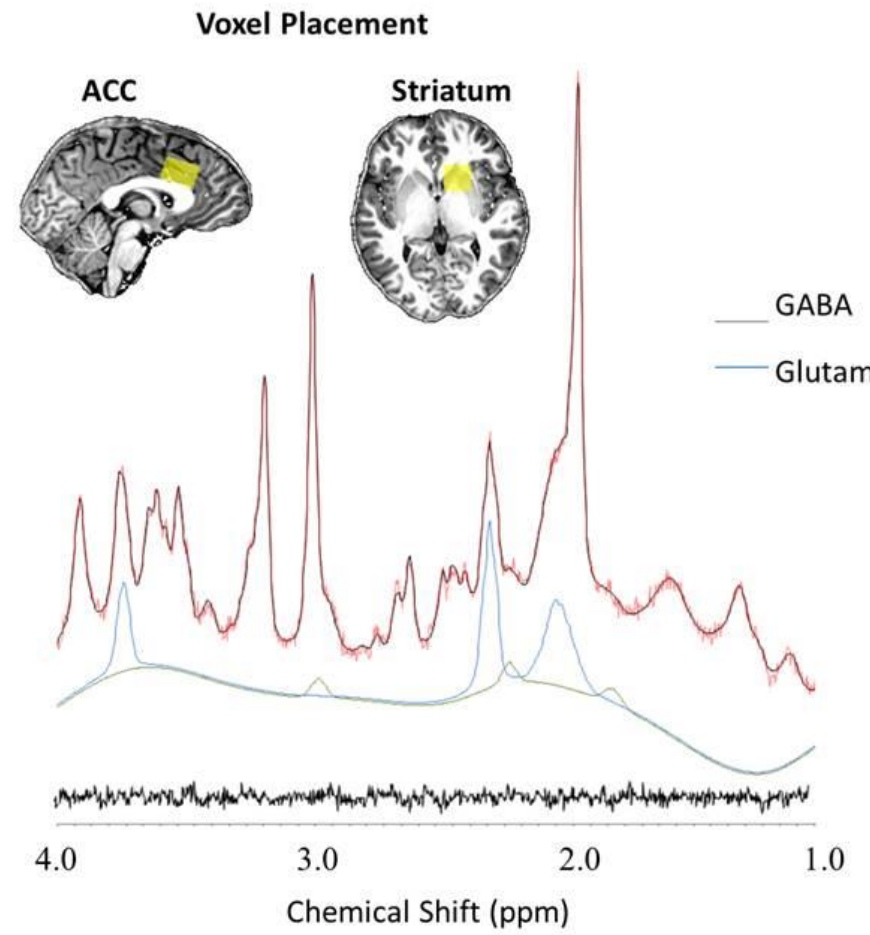

Chemical Shift (ppm)
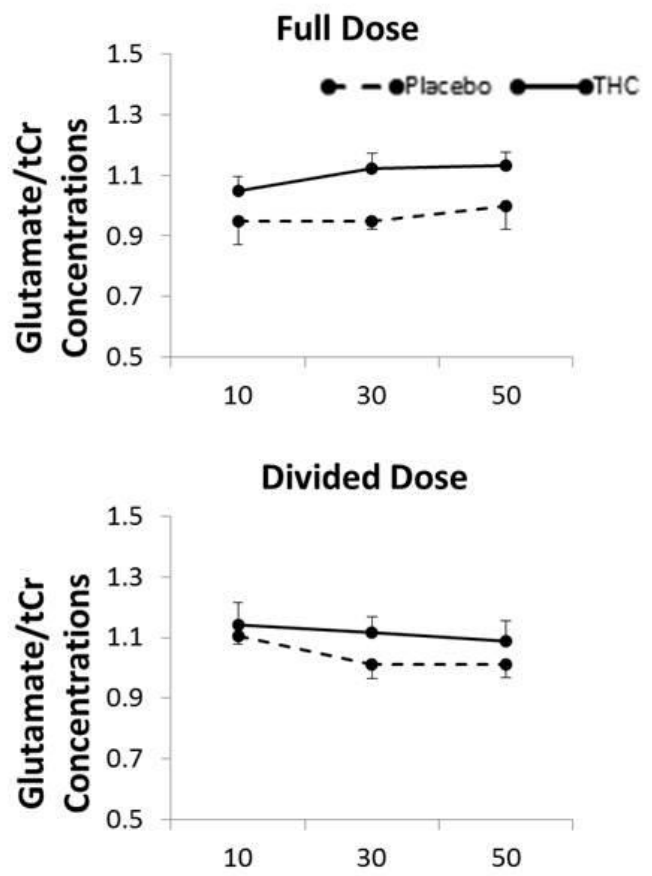

Time relative to smoking (minutes)

Figure 2. A. Example LC-Model fitted ${ }^{1} H$-MRS data recorded from one participant. The black line spectra corresponds to the phased ${ }^{1} \mathrm{H}$-MRS data with the LC-Model fits overlaid (red). The residual spectra (raw data minus the LC-Model fit) are displayed below the spectrum. B. Group 1 and Group 2 Mean (SE) Glu/tcr concentration levels in the striatum for both treatments (THC vs placebo) as a function of time relative to smoking $(10,30$, and 50 minutes).

\section{Functional Connectivity}

Figure 3 shows significant FC data in each treatment condition for the left NAc seed averaged over time. GLM revealed a main effect of Dose and a Dose x Treatment interaction (Table S4). Further GLM contrasts showed that relative to placebo, cannabis reduced functional connectivity with NAc seeds in both hemispheres in the full dose group, whereas no change was found in the divided dose group. Reductions in functional connectivity were prominent in broad areas of the frontal, temporal, parietal, and occipital lobes, a pattern typical of dopamine increase. However, GLM contrasts did not reveal any positive changes (THC > placebo) in functional connectivity. Table S5 shows the decrements in functional connectivity for the THC-placebo contrast in the full dose 
group. No significant differences were seen between the left and right NAc seed, so only left NAc seed results are shown. There were no main effects of Time or Time $x$ Treatment.
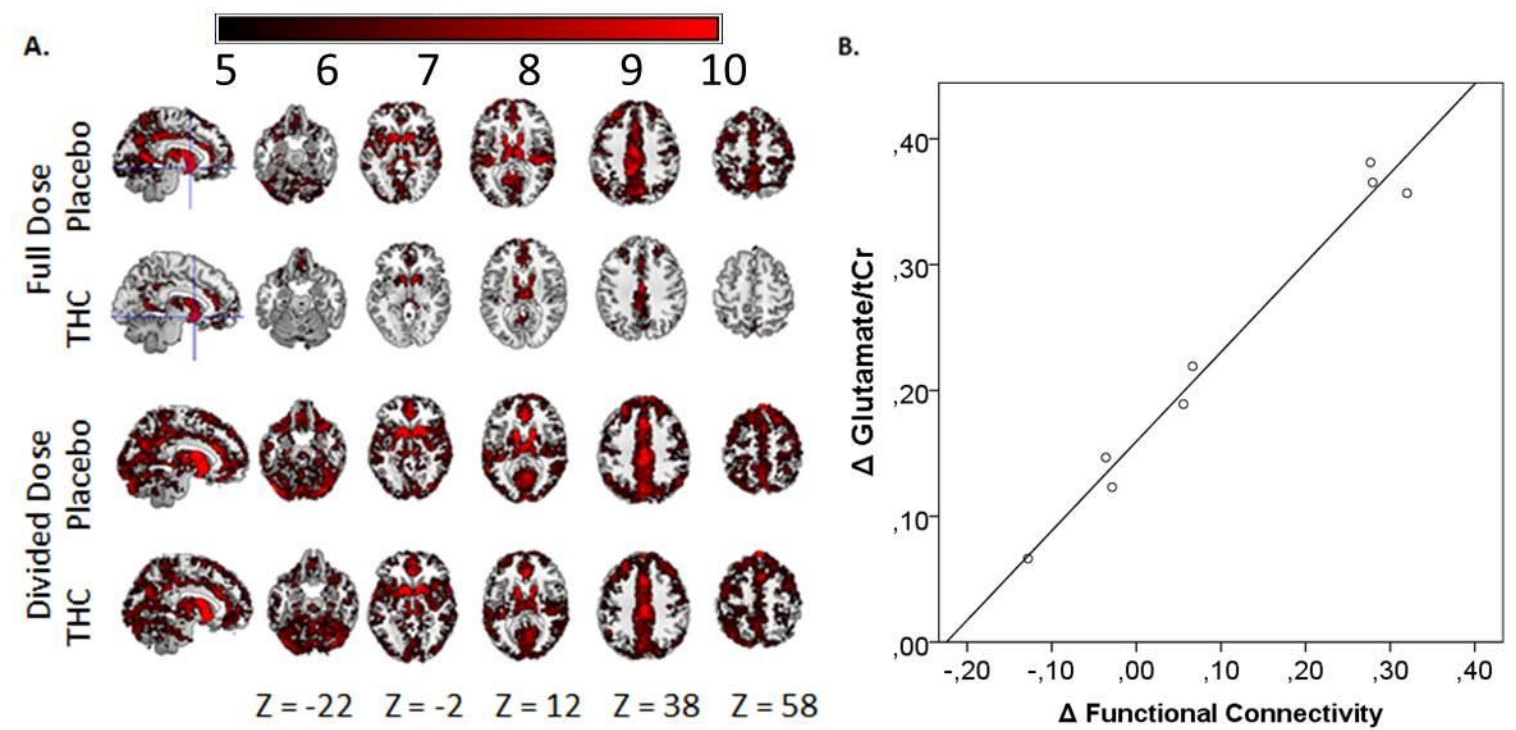

Figure 3. A. NAcc-related functional connectivity in the left hemispheres. Shown are thresholded Z-score maps of functional connectivity for each condition. The cross-hair indicates the seed ROI position. B. Example of correlation strength between absolute averaged striatal glutamate change concentration and change in functional connectivity between the left nucleus accumbens and the precentral gyrus ( $r=.989)$. Other areas showed correlations of similar strength.

Correlation of Subjective and Cognitive Effects, Metabolite and THC Concentrations, and Functional Connectivity

Voxel wise correlation analysis was conducted to evaluate the association between Pla > THC functional connectivity contrasts and changes in subjective high, attentional lapses, and glutamate changes in the striatum (Table S6). Correlation analysis revealed strong, positive correlations between glutamate changes in the striatum and functional connectivity between the left NAc and 6 cortical brain areas $(r>.975 ; P<.0001)$ (Figure 3 ; Table S6). Further analysis revealed a negative correlation between subjective high change scores $(r=-.987, P<.0001)$ and functional connectivity in the left NAc, and a positive correlation ( $r=.857, P=.006)$ between attentional lapse change scores and functional connectivity in the left NAc. Glutamate changes were not significantly correlated with changes in subjective high and attentional lapses. 


\section{Discussion}

The present study demonstrates the first attempt to assess acute influences of two different doses of cannabis on brain kinetics of glutamate, GABA, and dopamine, in relation to behavioral outcomes. Using a multimodal brain imaging approach, we showed significant neurometabolic and functional connectivity alterations during the absorption and elimination phase of THC in occasional cannabis users. These alterations were associated with changes in cognitive performance. Furthermore, these alterations were only evidenced when peak THC concentration levels were above $2 \mathrm{ng} / \mathrm{ml}$, suggesting a dose and concentration dependency.

MRS showed that, compared to placebo, THC increased glutamate concentrations in the striatum in the full dose group. These findings are compatible with previous human studies which have found reductions of glutamate-related metabolite concentrations in chronic cannabis users in the basal ganglia and dorsal striatum ${ }^{35}$. Specifically, the decrease found during post-acute studies could be postulated to be a result of increased glutamate activity during the acute phase

48 or an excessive down-regulation of glutamate signaling in chronic users ${ }^{31}$. Further support stems from rodent studies, which have found that use or exposure to THC disrupts glutamate signaling in the striatum, although the direction of the effect is mixed $34,49,50$.

MRS also showed that, compared to placebo, THC did not alter glutamate concentrations in the ACC. This is in accordance with a previous study by Sung, et al. ${ }^{51}$ who did not find glutamate + glutamine alterations in cannabis users compared to healthy controls. However, previous research has also found reductions of glutamate-related metabolite concentrations in adolescent cannabis users in the ACC $^{19,37}$. In addition, Prescot, et al. ${ }^{19}$ found significantly lower ACC GABA levels in adolescent cannabis users compared to non-using controls. This is in contrast to our study, which did not find any effects of THC on GABA in either the ACC or striatum. The absence of THC induced GABA changes could be due to inherent quantification challenges when assessing GABA concentration levels, arising from low brain concentration levels and metabolite signal overlap ${ }^{52,53}$. However in the present study, quantification precision of $\mathrm{GABA} / \mathrm{tCr}$, as estimated by Cramér-Rao Lower Bounds, overall surpassed the estimated signal change requirement, suggesting that null findings are not due to an inability to detect changes. Instead, the disagreement with studies could be due to the population under examination. Specifically, Prescot, et al. ${ }^{19}$ assessed GABA levels in adolescents with a mean age of 17.9, whereas our study assessed individuals with a mean age of 22.5. Although animal studies have suggested that acute THC administration increases GABA levels in the prefrontal cortex ${ }^{54}$ it has been suggested that these effects may be more pronounced in adolescence due to ongoing development ${ }^{55,56}$. Finally, the finding that THC did not alter GABA concentrations in the striatum is in accordance with a suggestion that GABAergic neurons in the $\mathrm{NAC}$ are less sensitive to THC than glutamatergic neurons ${ }^{50}$. 
In the full-dose group, THC decreased synchronicity between BOLD responses in the NAC and other areas of the brain. In particular, THC decreased functional connectivity between the NAC and areas of the frontal cortex (e.g., Brodmann area 6) and temporal cortex (e.g., Brodmann area 21 and 48), suggesting that THC directly affects the limbic reward circuit and decreases FC of brain areas in the circuit ${ }^{38,39}$, a marker of elevated dopamine. In accordance with this, previous studies have shown that acute THC induces dopamine release in the striatum ${ }^{57,58}$. The NAc receives it dopaminergic input from the VTA that is under inhibitory control of GABA interneurons on which presynaptic CB1 receptors are located. Stimulation of CB1 receptors by THC disinhibits the VTA which in turn increases dopamine levels in the $\mathrm{NAc}^{24}$.

THC induced increments in striatal dopamine correlated strongly with THC induced increments in striatal glutamate. Specifically, as treatment changes in FC between the NAc and areas in the frontal and parietal lobe increased, changes in striatal glutamate concentration increased. Previous evidence suggests that dopaminergic innervation of the NAc increases inhibitory GABAergic neurotransmission to the ventral pallidum, which leads to a reduction of GABAergic, inhibitory tone to the thalamus ${ }^{59}$. This process of disinhibition increases glutamatergic signaling from the thalamus to frontal cortex areas and subsequently to the $\mathrm{NAc}^{59}$, leading to increased striatal levels of glutamate as observed in the present study. In theory, increased stimulatory glutamatergic input to the NAc will add to the dopaminergic input from the VTA to the NAc and further strengthen the disinhibition of thalamic signaling in the corticostriatal circuitry. The strong correlation between changes in striatal FC and striatal glutamate is likely to reflect their interrelatedness as well as the similarity of their impact in the circuitry.

Significant correlations were also found between FC from the NAc and changes in subjective experience and cognitive function. A negative correlation was found between changes in FC of the NAc and the left (pre)cuneus and ratings of significant high on the VAS. Specifically, as treatment induced changes in FC between the NAc and the (pre)cuneus increased, changes in subjective high decreased. It has been suggested that the precuneus is involved in various processes including self-consciousness and self-related mental representations during rest ${ }^{60}$. THC is known to lower levels of consciousness during a cannabis induced subjective high ${ }^{61,62}$. Level of consciousness and feeling of subjective high are therefore anti-correlated during cannabis intoxication. The negative correlation between changes in FC and subjective high supports the notion that decrements in consciousness are associated with increments in subjective high. The (pre)cuneus has also been implicated in the default mode network ${ }^{63,64}$, a network of interacting brain areas that are correlated with each other during rest. Similarly, the default mode network has been found to be altered in cannabis users ${ }^{36,65}$, as well as during acute THC intoxication ${ }^{66,67}$. Furthermore, altered precuneus activity has been found in users of other dopaminergic drugs of 
abuse $^{68}$. Together this suggests that THC alters default mode network integrity via striatal dopaminergic activation, and that these changes may underlie subjective feelings of high.

A positive correlation was found between FC of the NAc and the left superior frontal gyrus (SFG) and the number of attentional lapses. Specifically, as treatment changes in FC between the NAc and the SFG increased, attentional performance decreased, as evidenced by an increase in number of attentional lapses. The SFG has been implicated in motor activity and higher cognitive functions, including working memory and attention ${ }^{69,70}$. Previous studies suggest that altered levels of dopamine impair cognitive performance ${ }^{71,72}$, and studies assessing cannabis users have found altered activity in this region $65,73,74$, as well as during acute THC intoxication 39,75 . Taken together this suggests that THC alters activity in the SFG via striatal dopaminergic as well as glutamatergic activation, and that these changes can impair cognitive performance.

Significant subjective, cognitive, and biological changes were only found in the full dose group, who reached average serum THC concentrations of $7 \mathrm{ng} / \mathrm{ml}$ at T-max after administration of the full THC dose. This is in contrast to the divided dose group, who reached average serum THC concentrations of $1.6 \mathrm{ng} / \mathrm{ml}$ at T-max. Previous research has suggested serum THC concentrations of less than $2 \mathrm{ng} / \mathrm{ml}$ do not affect cognitive performance ${ }^{10}$. This study extends previous research in demonstrating that at serum THC concentrations of less than $2 \mathrm{ng} / \mathrm{ml}$, brain functioning and metabolism are also not effected. As cannabis use becomes more popular, and access to medical cannabis grows, these findings are important in forensic settings when inferring impairment levels from THC levels in blood ${ }^{76}$.

In conclusion, administration of $300 \mu \mathrm{g} / \mathrm{kg}$ THC increased striatal glutamate concentrations, and increased dopamine as measured by functional connectivity. Average glutamate changes correlated strongly with functional connectivity alterations between the NAc and cortical areas. Additionally, THC induced changes in FC correlated with feelings of subjective high and decreased performance on an attention task. Taken together, these findings suggest that THC elicits subjective and cognitive alterations via increased striatal dopaminergic activity and loss of corticostriatal connectivity, which is associated with an increase in striatal glutamate. Finally, alterations are dose dependent and only evident after serum THC concentrations exceed $2 \mathrm{ng} / \mathrm{ml}$ at T-max. Future studies should address long-term neuroadaptions in cannabis users, which may play a role in substance addiction. 


\section{Chapter 2}

\section{References}

$1 \quad$ WHO. The Health and Social Effects of Nonmedical Cannabis Use. (2016).

2 EMCDDA. European Drug Report 2016: Trends and Developments. Publications Office of the European Union, Luxemborg (2016).

3 UN. World Drug Report 2016. United Nations publication (2016).

4 Johns, A. Psychiatric effects of cannabis. The British journal of psychiatry : the journal of mental science 178, 116-122 (2001).

5 Iversen, L. Cannabis and the brain. Brain : a journal of neurology 126, 1252-1270 (2003).

6 Green, B., Kavanagh, D. \& Young, R. Being stoned: a review of self-reported cannabis effects. Drug and alcohol review 22 , 453-460, doi:10.1080/09595230310001613976 (2003).

$7 \quad$ Hall, W. \& Solowij, N. Adverse effects of cannabis. Lancet (London, England) 352, 1611-1616, doi:10.1016/s01406736(98)05021-1 (1998).

8 Ramaekers, J. G., Berghaus, G., van Laar, M. \& Drummer, O. H. Dose related risk of motor vehicle crashes after cannabis use. Drug and alcohol dependence 73, 109-119 (2004).

9 Lundqvist, T. Cognitive consequences of cannabis use: comparison with abuse of stimulants and heroin with regard to attention, memory and executive functions. Pharmacology, biochemistry, and behavior 81, 319-330, doi:10.1016/j.pbb.2005.02.017 (2005).

Ramaekers, J. G. et al. Cognition and motor control as a function of Delta9-THC concentration in serum and oral fluid: limits of impairment. Drug and alcohol dependence 85, 114-122, doi:10.1016/j.drugalcdep.2006.03.015 (2006).

Glass, M., Dragunow, M. \& Faull, R. L. Cannabinoid receptors in the human brain: a detailed anatomical and quantitative autoradiographic study in the fetal, neonatal and adult human brain. Neuroscience 77, 299-318 (1997). Parsons, L. H. \& Hurd, Y. L. Endocannabinoid signalling in reward and addiction. Nature reviews. Neuroscience 16, 579594, doi:10.1038/nrn4004 (2015).

13 Burns, H. D. et al. [18F]MK-9470, a positron emission tomography (PET) tracer for in vivo human PET brain imaging of the cannabinoid-1 receptor. Proc Natl Acad Sci U S A 104, 9800-9805, doi:10.1073/pnas.0703472104 (2007). Gonzalez, R. Acute and non-acute effects of cannabis on brain functioning and neuropsychological performance. Neuropsychology review 17, 347-361, doi:10.1007/s11065-007-9036-8 (2007). Mathew, R. J., Wilson, W. H., Coleman, R. E., Turkington, T. G. \& DeGrado, T. R. Marijuana intoxication and brain activation in marijuana smokers. Life sciences 60, 2075-2089 (1997). Mathew, R. J., Wilson, W. H., Humphreys, D. F., Lowe, J. V. \& Wiethe, K. E. Regional cerebral blood flow after marijuana smoking. Journal of cerebral blood flow and metabolism : official journal of the International Society of Cerebral Blood Flow and Metabolism 12, 750-758, doi:10.1038/jcbfm.1992.106 (1992). doi:10.1097/01.wnr.0000075420.59944.20 (2003). Fusar-Poli, P. et al. Distinct effects of \{delta\}9-tetrahydrocannabinol and cannabidiol on neural activation during emotional processing. Archives of general psychiatry 66, 95-105, doi:10.1001/archgenpsychiatry.2008.519 (2009). Prescot, A. P., Renshaw, P. F. \& Yurgelun-Todd, D. A. gamma-Amino butyric acid and glutamate abnormalities in adolescent chronic marijuana smokers. Drug and alcohol dependence 129, 232-239, doi:10.1016/j.drugalcdep.2013.02.028 (2013).

Quickfall, J. \& Crockford, D. Brain Neuroimaging in Cannabis Use: A Review. The Journal of Neuropsychiatry and Clinical Neurosciences 18, 318-332, doi:10.1176/jnp.2006.18.3.318 (2006). Bloomfield, M. A., Ashok, A. H., Volkow, N. D. \& Howes, O. D. The effects of Delta9-tetrahydrocannabinol on the dopamine system. Nature 539, 369-377, doi:10.1038/nature20153 (2016). French, E. D. delta9-Tetrahydrocannabinol excites rat VTA dopamine neurons through activation of cannabinoid CB1 but not opioid receptors. Neuroscience letters 226, 159-162 (1997). Neuroreport 8, 649-652 (1997).

Lupica, C. R., Riegel, A. C. \& Hoffman, A. F. Marijuana and cannabinoid regulation of brain reward circuits. British journal of pharmacology 143, 227-234, doi:10.1038/sj.bjp.0705931 (2004).

Diana, M., Melis, M. \& Gessa, G. L. Increase in meso-prefrontal dopaminergic activity after stimulation of CB1 receptors by cannabinoids. Eur J Neurosci 10, 2825-2830 (1998). Fadda, P. et al. Cannabinoid self-administration increases dopamine release in the nucleus accumbens. Neuroreport 17, 1629-1632, doi:10.1097/01.wnr.0000236853.40221.8e (2006). Pistis, M. et al. $\Delta 9$-Tetrahydrocannabinol decreases extracellular GABA and increases extracellular glutamate and dopamine levels in the rat prefrontal cortex: an in vivo microdialysis study. Brain Research 948, 155-158 (2002).

$\mathrm{Ng}$ Cheong Ton, J. M. et al. The effects of delta 9-tetrahydrocannabinol on potassium-evoked release of dopamine in the rat caudate nucleus: an in vivo electrochemical and in vivo microdialysis study. Brain Res 451, 59-68 (1988). 


\section{Effects of cannabis on striatal glutamate and corticostriatal connectivity}

Gessa, G. L., Melis, M., Muntoni, A. L. \& Diana, M. Cannabinoids activate mesolimbic dopamine neurons by an action on cannabinoid CB1 receptors. European journal of pharmacology 341, 39-44 (1998).

Navarro, M. et al. An acute dose of delta 9-tetrahydrocannabinol affects behavioral and neurochemical indices of mesolimbic dopaminergic activity. Behav Brain Res 57, 37-46 (1993).

Colizzi, M., McGuire, P., Pertwee, R. G. \& Bhattacharyya, S. Effect of cannabis on glutamate signalling in the brain: A systematic review of human and animal evidence. Neuroscience and Biobehavioral Reviews 64, 359-381, doi:10.1016/j.neubiorev.2016.03.010 (2016).

Schlicker, E. \& Kathmann, M. Modulation of transmitter release via presynaptic cannabinoid receptors. Trends in pharmacological sciences 22, 565-572 (2001).

Pertwee, R. G. The diverse CB1 and CB2 receptor pharmacology of three plant cannabinoids: delta9tetrahydrocannabinol, cannabidiol and delta9-tetrahydrocannabivarin. British journal of pharmacology 153, 199-215, doi:10.1038/sj.bjp.0707442 (2008).

Galanopoulos, A., Polissidis, A., Papadopoulou-Daifoti, Z., Nomikos, G. G. \& Antoniou, K. Delta(9)-THC and WIN55,212-2 affect brain tissue levels of excitatory amino acids in a phenotype-, compound-, dose-, and region-specific manner. Behav Brain Res 224, 65-72, doi:10.1016/j.bbr.2011.05.018 (2011).

Chang, L., Cloak, C., Yakupov, R. \& Ernst, T. Combined and independent effects of chronic marijuana use and HIV on brain metabolites. Journal of neuroimmune pharmacology : the official journal of the Society on Neurolmmune Pharmacology 1, 65-76, doi:10.1007/s11481-005-9005-z (2006).

Muetzel, R. L. et al. In vivo (1)H magnetic resonance spectroscopy in young-adult daily marijuana users(). Neurolmage : Clinical 2, 581-589 (2013).

Prescot, A. P., Locatelli, A. E., Renshaw, P. F. \& Yurgelun-Todd, D. A. Neurochemical alterations in adolescent chronic marijuana smokers: a proton MRS study. Neurolmage 57, 69-75, doi:10.1016/j.neuroimage.2011.02.044 (2011).

Ramaekers, J. et al. Methylphenidate reduces functional connectivity of nucleus accumbens in brain reward circuit. Psychopharmacology (Berl) 229, 219-226, doi:10.1007/s00213-013-3105-x (2013).

Ramaekers, J. G. et al. Cannabis and cocaine decrease cognitive impulse control and functional corticostriatal connectivity in drug users with low activity DBH genotypes. Brain Imaging and Behavior 10, 1254-1263 (2016).

Theunissen, E. L. et al. Neurophysiological functioning of occasional and heavy cannabis users during THC intoxication. Psychopharmacology (Berl) 220, 341-350 (2012).

Ramaekers, J. G. et al. Cannabis and tolerance: acute drug impairment as a function of cannabis use history. Scientific Reports 6 (2016).

Frahm, J. et al. Localized high-resolution proton NMR spectroscopy using stimulated echoes: initial applications to human brain in vivo. Magnetic resonance in medicine 9, 79-93 (1989).

Chao-Gan, Y. \& Yu-Feng, Z. DPARSF: A MATLAB Toolbox for "Pipeline" Data Analysis of Resting-State fMRI. Frontiers in systems neuroscience 4, 13, doi:10.3389/fnsys.2010.00013 (2010).

Di Martino, A. et al. Functional connectivity of human striatum: a resting state FMRI study. Cerebral cortex (New York, N.Y. : 1991) 18, 2735-2747, doi:10.1093/cercor/bhn041 (2008).

Kelly, C. et al. L-dopa modulates functional connectivity in striatal cognitive and motor networks: a double-blind placebocontrolled study. The Journal of neuroscience : the official journal of the Society for Neuroscience 29, 7364-7378, doi:10.1523/jneurosci.0810-09.2009 (2009).

Rosner, B. Fundamentals of Biostatistics. 6 edn, (Thomson-Brooks/Cole, 2006).

Dinges, D. F. \& Powell, J. W. Microcomputer analyses of performance on a portable, simple visual RT task during sustained operations. Behavior Research Methods, Instruments, \& Computers 17, 652-655, doi:10.3758/bf03200977 (1985).

Sampedro, F. et al. Assessing the Psychedelic "After-Glow" in Ayahuasca Users: Post-Acute Neurometabolic and Functional Connectivity Changes Are Associated with Enhanced Mindfulness Capacities. The international journal of neuropsychopharmacology 20, 698-711, doi:10.1093/ijnp/pyx036 (2017).

Brown, T. M., Brotchie, J. M. \& Fitzjohn, S. M. Cannabinoids decrease corticostriatal synaptic transmission via an effect on glutamate uptake. The Journal of neuroscience : the official journal of the Society for Neuroscience 23, 11073-11077 (2003).

Sano, K. et al. Delta 9-tetrahydrocannabinol-induced catalepsy-like immobilization is mediated by decreased 5-HT neurotransmission in the nucleus accumbens due to the action of glutamate-containing neurons. Neuroscience 151, 320328, doi:10.1016/j.neuroscience.2007.10.026 (2008).

Sung, Y.-H. et al. Decreased frontal N-acetylaspartate levels in adolescents concurrently using both methamphetamine and marijuana. Behavioural brain research 246, 154-161, doi:https://doi.org/10.1016/j.bbr.2013.02.028 (2013).

Puts, N. A. J. \& Edden, R. A. E. In vivo magnetic resonance spectroscopy of GABA: a methodological review. Progress in nuclear magnetic resonance spectroscopy 60, 29-41, doi:10.1016/j.pnmrs.2011.06.001 (2012).

Shungu, D. C. et al. Brain $\gamma$-aminobutyric acid (GABA) detection in vivo with the J-editing (1) H MRS technique: a comprehensive methodological evaluation of sensitivity enhancement, macromolecule contamination and test-retest reliability. NMR in biomedicine 29, 932-942, doi:10.1002/nbm.3539 (2016). 


\section{Chapter 2}

Pistis, M. et al. Delta(9)-tetrahydrocannabinol decreases extracellular GABA and increases extracellular glutamate and dopamine levels in the rat prefrontal cortex: an in vivo microdialysis study. Brain research 948, 155-158 (2002).

Sneider, J. T., Mashhoon, Y. \& Silveri, M. M. A Review of Magnetic Resonance Spectroscopy Studies in Marijuana using Adolescents and Adults. Journal of addiction research \& therapy Suppl 4, 010, doi:10.4172/2155-6105.S4-010 (2013).

Silveri, M. M. et al. Frontal Lobe GABA Levels during Adolescence: Associations with Impulsivity and Response Inhibition. Biological psychiatry 74, 296-304, doi:10.1016/j.biopsych.2013.01.033 (2013).

Bossong, M. G. et al. Further human evidence for striatal dopamine release induced by administration of $\Delta 9$ tetrahydrocannabinol (THC): selectivity to limbic striatum. Psychopharmacology 232, 2723-2729, doi:10.1007/s00213015-3915-0 (2015).

Bossong, M. G. et al. Delta 9-tetrahydrocannabinol induces dopamine release in the human striatum. Neuropsychopharmacology 34, 759-766, doi:10.1038/npp.2008.138 (2009).

Pierce, R. C. \& Kumaresan, V. The mesolimbic dopamine system: the final common pathway for the reinforcing effect of drugs of abuse? Neurosci Biobehav Rev 30, 215-238, doi:10.1016/j.neubiorev.2005.04.016 (2006).

Cavanna, A. E. \& Trimble, M. R. The precuneus: a review of its functional anatomy and behavioural correlates. Brain 129, 564-583 (2006).

Koethe, D. et al. Disturbances of visual information processing in early states of psychosis and experimental delta-9tetrahydrocannabinol altered states of consciousness. Schizophrenia research 88, 142-150, doi:10.1016/j.schres.2006.07.023 (2006).

Vadhan, N. P., Corcoran, C. M., Bedi, G., Keilp, J. G. \& Haney, M. Acute effects of smoked marijuana in marijuana smokers at clinical high-risk for psychosis: A preliminary study. Psychiatry research 257, 372-374, doi:10.1016/j.psychres.2017.07.070 (2017).

Greicius, M. D., Krasnow, B., Reiss, A. L. \& Menon, V. Functional connectivity in the resting brain: a network analysis of the default mode hypothesis. Proceedings of the National Academy of Sciences of the United States of America 100, 253258, doi:10.1073/pnas.0135058100 (2003).

Utevsky, A. V., Smith, D. V. \& Huettel, S. A. Precuneus Is a Functional Core of the Default-Mode Network. The Journal of Neuroscience 34, 932-940, doi:10.1523/JNEUROSCI.4227-13.2014 (2014).

Cheng, H. et al. Resting state functional magnetic resonance imaging reveals distinct brain activity in heavy cannabis users - a multi-voxel pattern analysis. Journal of psychopharmacology (Oxford, England) 28, 1030-1040, doi:10.1177/0269881114550354 (2014).

Bossong, M. G. et al. Default mode network in the effects of Delta9-Tetrahydrocannabinol (THC) on human executive function. PloS one 8, e70074, doi:10.1371/journal.pone.0070074 (2013).

Klumpers, L. E. et al. Manipulating brain connectivity with delta(9)-tetrahydrocannabinol: a pharmacological resting state FMRI study. Neurolmage 63, 1701-1711, doi:10.1016/j.neuroimage.2012.07.051 (2012).

Moeller, S. J., London, E. D. \& Northoff, G. Neuroimaging markers of glutamatergic and GABAergic systems in drug addiction: Relationships to resting-state functional connectivity. Neuroscience \& Biobehavioral Reviews 61, 35-52, doi:https://doi.org/10.1016/j.neubiorev.2015.11.010 (2016).

Boisgueheneuc, F. d. et al. Functions of the left superior frontal gyrus in humans: a lesion study. Brain 129, 3315-3328 (2006).

Nagahama, Y. et al. Transient neural activity in the medial superior frontal gyrus and precuneus time locked with attention shift between object features. Neurolmage 10, 193-199, doi:10.1006/nimg.1999.0451 (1999).

Cools, R. \& D'Esposito, M. Inverted-U-shaped dopamine actions on human working memory and cognitive control. Biological psychiatry 69, e113-e125 (2011).

Prashad, S. \& Filbey, F. M. Cognitive motor deficits in cannabis users. Current opinion in behavioral sciences 13, 1-7 (2017). Orr, C. et al. Altered resting-state connectivity in adolescent cannabis users. The American journal of drug and alcohol abuse 39, 372-381, doi:10.3109/00952990.2013.848213 (2013).

van Hell, H. H. et al. Chronic effects of cannabis use on the human reward system: an fMRI study. European neuropsychopharmacology : the journal of the European College of Neuropsychopharmacology 20, 153-163, doi:10.1016/j.euroneuro.2009.11.010 (2010).

Weinstein, A. et al. Brain imaging study of the acute effects of Delta9-tetrahydrocannabinol (THC) on attention and motor coordination in regular users of marijuana. Psychopharmacology (Berl) 196, 119-131, doi:10.1007/s00213-007-0940-7 (2008).

Khiabani, H. Z., Bramness, J. G., Bjorneboe, A. \& Morland, J. Relationship between THC concentration in blood and impairment in apprehended drivers. Traffic injury prevention 7, 111-116, doi:10.1080/15389580600550172 (2006). 


\section{Supplemental Information}

\section{Material and Methods}

Participants. In total, 25 participants entered the study. Five participants dropped out for various non-study related reasons. Participants were recruited through advertisements around Maastricht University. Inclusion criteria were: age, 18-40 years; occasional cannabis use, ranging between 1 time a month and 3 times a week for the past year; normal weight, body mass index between 18 and $28 \mathrm{~kg} / \mathrm{m} 2$; free from psychotropic medication; good physical health, including absence of major medical, endocrine, and neurological conditions; and written informed consent. Exclusion criteria were: history of drug abuse (other than the use of cannabis) or addiction, which were determined by medical questionnaires and examination; pregnancy or lactation; health issues including hypertension (diastolic >90 and systolic $>140$ ), cardiac dysfunction, and liver dysfunction; current or history of psychiatric disorders; previous experience of serious side effects to cannabis; and MRI contraindications. Before inclusion, subjects were screened and examined by a study physician, who checked for general health, conducted a resting ECG, and took blood and urine samples in which hematology, clinical chemistry, urine, and virology analyses were conducted. Participant demographic data can be found in Table S5.

A permit for obtaining, storing, and administering cannabis was obtained from the Dutch Drug Enforcement Administration. Participants were financially compensated for their participation in the study.

Administration. Both treatments were administered through a Volcano vaporizer (Storz \& Bickel Volcano ${ }^{\circledR}$ ), with participants inhaling equal amounts of each while lying in the MRI machine. The treatments were vaporized at $225^{\circ} \mathrm{C}$ and the vapor was stored in a polythene bag equipped with a mouth piece. Participants were instructed to place the mouth piece to their lips and inhale deeply, holding their breath for 10 seconds, and then exhaling. Participants repeated this procedure until balloon was empty. Participants were instructed to inhale the entire volume of the balloon within 5 minutes, according to a standardized procedure.

Procedures. Participants were familiarized with the test day procedures on a separate training day prior to the treatment conditions. Participants were instructed to refrain from drug use ( $\geq 7$ days) and alcohol use ( $\geq 24$ hours) prior to their testing day. On arrival on a test day, absence of drug and alcohol were assessed via a urine drug screen and a breath alcohol screen. An additional pregnancy test was given if participants were female. If all tests were found to be negative, participants were allowed to proceed, and a venal catheter was placed.

MRS Data Acquisition and Quality. Single-voxel proton magnetic resonance spectroscopy (MRS) measurements were performed on a MAGNETOM 7T MR scanner (Siemens Healthineers, 
Erlangen, Germany) with a whole-body gradient set (SC72; maximum amplitude, $70 \mathrm{mT} / \mathrm{m}$; maximum slew rate, $200 \mathrm{~T} / \mathrm{m} / \mathrm{s}$ ) and using an single-channel transmit/32-channel receive head coil (Nova Medical, Wilmington, MA, USA). Spectroscopic voxels of interest were placed by a trained operator at the Anterior Cingulate Cortex (ACC) (voxel size $=25 \times 20 \times 17 \mathrm{~mm}^{3}$ ) and the right striatum (voxel size $=20 \times 20 \times 20 \mathrm{~mm}^{3}$ ). Spectra were acquired with stimulated echo acquisition mode $(S T E A M){ }^{1}$ sequence using the following parameters: $T E=6.0 \mathrm{~ms}, \mathrm{TM}=10.0 \mathrm{~ms}, \mathrm{TR}=5.0 \mathrm{~s}$, $\mathrm{NA}=64$, flip angle $=90^{\circ}, \mathrm{RF}$ bandwidth $=4.69 \mathrm{kHz}$, RF centred at $2.4 \mathrm{ppm}$, receive bandwidth $=$ $4.0 \mathrm{kHz}$, vector size $=2048,16$-step phase cycling, acquisition time $=5: 20 \mathrm{~min}$. Water suppression was achieved by variable power RF pulses with optimised relaxation delays (VAPOR) ${ }^{2}$. In addition, a complete phase cycle of measurements was acquired without the water suppression RF pulses to record a water peak reference for eddy current correction ${ }^{3}$ and absolute metabolite concentration calibration ${ }^{4,5}$. Before the spectroscopy measurements, a 3D-GRE dual-echo fieldmap $\left(\mathrm{TE}_{1}=1.00 \mathrm{~ms}, \mathrm{TE}_{2}=2.98 \mathrm{~ms}\right.$, $\mathrm{TR}=20.0 \mathrm{~ms}$, flip angle $=8^{\circ}$, voxel size $=3 \mathrm{~mm}$ isotropic, matrix size $=84 \times 84 \times 56$, bandwidth $=1450 \mathrm{~Hz} /$ pixel, acquisition time $=2: 24 \mathrm{~min}$ ) was acquired and used calculate the shim currents required to homogenise the static magnetic field in the spectroscopic voxels of interest.

The spectra were analysed with LCModel version 6.3-1H using a GAMMA ${ }^{6}$ simulated basis set which includes Alanine (Ala), Ascorbic Acid (Asc), Aspartate (Asp), Creatine (Cr), y-Aminobutyric Acid (GABA), Glucose (Glc), Glutamate (Glu), Glutamine (Gln), Glycerophosphocholine (GPC), Glutathione (GSH), Glycine (Glyc), Lactate (Lac), Myo-Inositol (ml), N-Acetyl Aspartate (NAA), NAcetyl Aspartyl Glutamate (NAAG), Phosphocreatine (PCr), Phosphorylcholine (PCh), Phosphorylethanolamine (PE), Scyllo-Inositol (Scyllo), and Taurine (Tau) ${ }^{7}$. The metabolite basis set also includes an in vivo Macromolecules (MMol) spectrum which was collected using a metabolites suppressed double inversion recovery (DIR) STEAM with the same parameters as above and $\mathrm{Tl}_{1}=2.09 \mathrm{~s}$ and $\mathrm{TI}_{2}=0.52 \mathrm{~s}^{8}$.

Anatomical ( $T_{1}$-weighted) images were acquired using magnetisation-prepared 2 rapid acquisition gradient-echo (MP2RAGE) ${ }^{9}$ sequence $\left(\mathrm{TR}=4.5 \mathrm{~s}, \mathrm{TE}=2.39 \mathrm{~ms}, \mathrm{Tl}_{1}=0.90 \mathrm{~s}, \mathrm{Tl}_{2}=2.75\right.$ s, flip angle ${ }_{1}=5^{\circ}$, flip angle $e_{2}=3^{\circ}$, voxel size $=0.9 \mathrm{~mm}$ isotropic, matrix size $=256 \times 256 \times 192$, phase partial Fourier $=6 / 8$, GRAPPA factor $=3$ with 24 reference lines, bandwidth $=250 \mathrm{~Hz} /$ pixel, acquisition time $=6: 00 \mathrm{~min})$. Tissue probability maps for grey matter $(G M)$, white matter (WM) and cerebrospinal fluid (CSF) were generated from the $\mathrm{T}_{1}$-weighted anatomical images using FSLFAST ${ }^{10}$. To ensure data quality and reliable metabolite estimation, only absolute metabolite values with a Cramer-Rao lower bound (CRLB) below 20\% and a signal-to-noise ratio greater than 10 were considered (Provencher, 2001). Furthermore, due to inherent quantification challenges when assessing GABA concentration levels ${ }^{11,12}$, it was assessed as to what magnitude a change in GABA concentration levels would have to be in order to be reliably detectable. Mean percentage 
signal change was calculated per time point and compared to required signal change (estimated by CRLBs). Over $80 \%$ of GABA measurements surpassed this threshold, suggesting reliable GABA quantification precision. An example spectra can be found in Figure 3.

Psychomotor vigilance task. This study used a shortened, 5-minute version of the task on three consecutive time points after treatment administration. The outcome variables of the PVT are: mean RT, the mean RT for all trials; optimum response domain, the fastest $10 \%$ of response times for all trials (i.e. average of the fastest 10\% RT); number of lapses, the number of response times greater than or equal to 500 ms for all trials (i.e. lapse); and lapse domain, the slowest 10\% of reciprocal response times for all trials (i.e. average of the slowest $10 \% 1 / \mathrm{RT}$ ) ${ }^{13}$. For this study, only number of lapses was considered.

Table S1. Mean subject characteristics (SE; mix-max) and history of drug use for occasional cannabis users that completed the study $(N=20)$.

\begin{tabular}{|c|l|l|l|l|l|l|l|}
\hline Group & Age & $\begin{array}{l}\text { History of } \\
\text { cannabis } \\
\text { use } \\
\text { (years) }\end{array}$ & $\begin{array}{l}\text { Frequency } \\
\text { of cannabis } \\
\text { use }(\text { per } \\
\text { month) }\end{array}$ & $\begin{array}{l}\text { Alcohol } \\
\text { consumption } \\
\text { (per week) }\end{array}$ & $\begin{array}{l}\text { Caffeine } \\
\text { consumption } \\
\text { (per week) }\end{array}$ & $\begin{array}{l}\text { Nicotine } \\
\text { consumption } \\
\text { (per week) }\end{array}$ & $\begin{array}{l}\text { Occasional use } \\
\text { of other drugs* } \\
\text { (percentage of } \\
\text { subjects) }\end{array}$ \\
\hline 1 & $\begin{array}{l}22.5 \\
(.86 ; \\
19- \\
28)\end{array}$ & $\begin{array}{l}5.2(.90 ; \\
2-11)\end{array}$ & $4.5(.82 ; 1-8)$ & $\begin{array}{l}8.4(2.29 ; 1- \\
20)\end{array}$ & $\begin{array}{l}8.5(2.48 ; 0- \\
21)\end{array}$ & $\begin{array}{l}18.9(7.41 ; 0- \\
70)\end{array}$ & $80 \%$ \\
\hline 2 & $\begin{array}{l}21.2 \\
(.85 ; \\
18-\end{array}$ & $\begin{array}{l}4.5(.64 ; \\
2-9)\end{array}$ & $6.2(1.26 ; 2-$ & $5.1(1.43 ; 0-$ & $8.4(2.72 ; 0-$ & $7(4.17 ; 0-$ & $35)$ \\
$12)$ & $28)$ & & & $60 \%$ \\
\hline
\end{tabular}

*including MDMA, psychedelic mushrooms, amphetamine, cocaine, LSD, and ketamine 


\section{Chapter 2}

Table S2. Testing day schedule

\begin{tabular}{|c|c|c|c|}
\hline $\begin{array}{l}\text { Time after first treatment } \\
\text { (minutes) }\end{array}$ & & Procedure & \\
\hline \multirow[t]{5}{*}{ Baseline } & 0 & $\begin{array}{l}\text { Urine sample (drug screen; } \\
\text { pregnancy screen) }\end{array}$ & \\
\hline & 0 & Alcohol breath test & \\
\hline & 0 & Vital signs & \\
\hline & 0 & Questionnaires & \\
\hline & 0 & Blood sample (S1) & \\
\hline \multicolumn{4}{|l|}{ In Scanner } \\
\hline & 0 & Anatomical scan & \\
\hline & & Full Dose & Divided Dose \\
\hline & 0 & Administration $(300 \mu \mathrm{g} / \mathrm{kg})$ & Administration $(100 \mu \mathrm{g} / \mathrm{kg})$ \\
\hline & 1 & MRS (ACC); PVT & \\
\hline & 6 & Blood sample (S2) & \\
\hline & 8 & MRS (striatum) & \\
\hline & 15 & Resting state scan & \\
\hline & 20 & Questionnaires & \\
\hline & & & Administration $(100 \mu \mathrm{g} / \mathrm{kg})$ \\
\hline & 22 & MRS (ACC); PVT & \\
\hline & 28 & Blood sample (S3) & \\
\hline & 30 & MRS (striatum) & \\
\hline & 36 & Resting state scan & \\
\hline & 42 & Questionnaires & \\
\hline & & & Administration $(100 \mu \mathrm{g} / \mathrm{kg})$ \\
\hline & 44 & MRS (ACC); PVT & \\
\hline & 50 & Blood sample (S4) & \\
\hline & 52 & MRS (striatum) & \\
\hline & 58 & Resting state scan & \\
\hline \multicolumn{4}{|l|}{ Outside of scanner } \\
\hline & 68 & Questionnaires & \\
\hline & 72 & Blood sample (S5) & \\
\hline & 75 & Sober up & \\
\hline
\end{tabular}


Effects of cannabis on striatal glutamate and corticostriatal connectivity

Table S3. Group 1 and Group 2 mean (SE) Glu/tcr and GABA/tcr concentrations. Values are not shown for group 2, timepoint 3, striatal GABA/tcr due to data not meeting acception criteria. ${ }^{*} \mathrm{P}<.05$

\begin{tabular}{|c|c|c|c|c|c|c|c|c|c|}
\hline & \multirow[b]{3}{*}{ Time } & \multicolumn{4}{|l|}{ Full Dose } & \multicolumn{4}{|c|}{ Divided Dose } \\
\hline & & \multicolumn{2}{|l|}{ Glu/tcr } & \multicolumn{2}{|l|}{ GABA/tcr } & \multicolumn{2}{|l|}{ Glu/tcr } & \multicolumn{2}{|l|}{ GABA/tcr } \\
\hline & & THC & Placebo & THC & Placebo & THC & Placebo & THC & Placebo \\
\hline \multirow{3}{*}{ 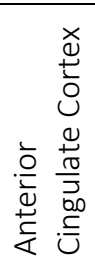 } & 1 & $1.09(.06)$ & $\begin{array}{l}1.03 \\
(.05)\end{array}$ & $.19(.01)$ & $.15(.01)$ & $1.11(.04)$ & $\begin{array}{l}1.09 \\
(.03)\end{array}$ & $.14(.01)$ & $.15(.02)$ \\
\hline & 2 & $1.09(.04)$ & $\begin{array}{l}1.07 \\
(.03)\end{array}$ & $.16(.01)$ & $.14(.01)$ & $1.08(.03)$ & $\begin{array}{l}1.09 \\
(.03)\end{array}$ & $.14(.01)$ & $.16(.01)$ \\
\hline & 3 & $1.08(.06)$ & $\begin{array}{l}1.08 \\
(.06) \\
\end{array}$ & $.18(.01)$ & $.15(.02)$ & $1.08(.03)$ & $\begin{array}{l}1.08 \\
(.04) \\
\end{array}$ & $.15(.01)$ & $.16(.02)$ \\
\hline \multirow{3}{*}{ 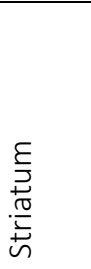 } & 1 & $1.05(.04)$ & $.95(.08)$ & $.22(.01)$ & $.22(.05)$ & $1.10(.07)$ & $\begin{array}{l}1.09 \\
(.03)\end{array}$ & $.21(.04)$ & $.24(.03)$ \\
\hline & 2 & $1.12(.05)^{*}$ & $.95(.03)$ & $.25(.04)$ & $.21(.03)$ & $1.13(.05)$ & $\begin{array}{l}1.00 \\
(.04) \\
\end{array}$ & $.19(.04)$ & $.19(.03)$ \\
\hline & 3 & $1.13(.04)^{*}$ & $.99(.08)$ & $.21(.03)$ & $.19(.03)$ & $1.06(.07)$ & $\begin{array}{l}1.00 \\
(.05)\end{array}$ & - & - \\
\hline
\end{tabular}




\section{Chapter 2}

Table S4. Areas displaying significant main effect of Dose, and significant effect of Treatment X Dose

\section{Main effect of Dose}

\begin{tabular}{|c|c|c|c|c|c|c|c|}
\hline Cluster & $\mathrm{R}$ or $\mathrm{L}$ & BA & & $\mathrm{x}$ & $\mathrm{y}$ & $\mathrm{z}$ & $P$ value $F_{34.2} F W E$ corrected \\
\hline Medial frontal gyrus & $\mathrm{L}$ & 32 & 9 & -10 & 32 & 32 & .000 \\
\hline Superior frontal gyrus & L & 9 & 33 & -14 & 50 & 46 & .000 \\
\hline Medial frontal gyrus & $\mathrm{L}$ & 9 & 5 & 0 & 52 & 38 & .000 \\
\hline Medial frontal gyrus & $\mathrm{R}$ & 9 & 12 & 6 & 56 & 34 & .000 \\
\hline Medial frontal gyrus & L & 8 & 11 & -4 & 34 & 62 & .000 \\
\hline Medial frontal gyrus & $\mathrm{R}$ & 8 & 9 & 8 & 28 & 64 & .000 \\
\hline Medial frontal gyrus & $\mathrm{R}$ & & 32 & 12 & 48 & 48 & .000 \\
\hline Superior frontal gyrus & $\mathrm{R}$ & 8 & 13 & 18 & 20 & 62 & .000 \\
\hline Supplementary motor area & $\mathrm{L}$ & 8 & 4 & -14 & 20 & 64 & .000 \\
\hline Supplementary motor area & $\mathrm{R}$ & 6 & 19 & 4 & 6 & 66 & .000 \\
\hline Supplementary motor area & $\mathrm{R}$ & 6 & 6 & 12 & 14 & 72 & .000 \\
\hline Supplementary motor area & $\mathrm{R}$ & 6 & 7 & 4 & 14 & 72 & .000 \\
\hline Supplementary motor area & $\mathrm{R}$ & 6 & 6 & 12 & 6 & 70 & .000 \\
\hline Superior temporal gyrus & $\mathrm{R}$ & 21 & 10 & 56 & 2 & -10 & .000 \\
\hline Middle temporal gyrus & $\mathrm{R}$ & 21 & 4 & 48 & -36 & 6 & .000 \\
\hline Middle temporal gyrus & $\mathrm{R}$ & 37 & 6 & 44 & -60 & 4 & .000 \\
\hline Fusiform gyrus & $\mathrm{R}$ & 37 & 12 & 38 & -56 & -20 & .000 \\
\hline Cerebellum & $\mathrm{L}$ & 37 & 4 & -28 & -32 & -26 & .000 \\
\hline Middle temporal gyrus & L & 48 & 4 & -48 & -26 & 2 & .000 \\
\hline Supramarginal gyrus & $\mathrm{R}$ & 48 & 7 & 50 & -26 & 26 & .000 \\
\hline Putamen & $\mathrm{R}$ & 48 & 3 & 24 & 8 & 4 & .000 \\
\hline Inferior temporal gyrus & $\mathrm{L}$ & 20 & 12 & -52 & -14 & -24 & .000 \\
\hline Hippocampus & L & 20 & 5 & -32 & -16 & -16 & .000 \\
\hline Cerebellum & $\mathrm{R}$ & 20 & 15 & 28 & -30 & -28 & .000 \\
\hline
\end{tabular}




\begin{tabular}{|c|c|c|c|c|c|}
\hline Precuneus & L & 5 & 19 & -4 & -4866 \\
\hline Fusiform gyrus & L & 30 & 13 & -22 & $-32-20$ \\
\hline Fusiform gyrus & $\mathrm{R}$ & 19 & 13 & 32 & $-78-16$ \\
\hline Inferior occipital cortex & $\mathrm{R}$ & 19 & 17 & 32 & $-82-2$ \\
\hline Inferior occipital cortex & $R$ & 19 & 3 & 32 & $-84-14$ \\
\hline Cerebellum & L & 19 & 3 & -32 & $-62-24$ \\
\hline Cerebellum & L & 19 & 3 & -44 & $-66-26$ \\
\hline Cerebellum & $\mathrm{R}$ & 18 & 5 & 14 & $-76-18$ \\
\hline Inferior occipital cortex & $\mathrm{R}$ & 18 & 3 & 28 & $-86-6$ \\
\hline Midcingulate area & $\mathrm{R}$ & & 4 & 8 & -1046 \\
\hline Cerebellum & L & & 10 & -48 & $-58-42$ \\
\hline Vermis & & & 6 & -2 & $-52-38$ \\
\hline
\end{tabular}

Dose X Treatment

Cluster $\quad$ R or L BA $\quad x \quad y \quad z \quad P$ value F $F_{11.52}$ FWE cluster corrected

\begin{tabular}{llllllll}
\hline Inferior frontal gyrus, pars triangularis $\quad$ L & 45 & 37 & -42 & 22 & 18 & .000
\end{tabular}

$\begin{array}{llllllll}\text { Insula } & \mathrm{L} & 47 & 21 & -24 & 26 & 2 & .013\end{array}$

$\mathrm{MNI}$ coordinates of peak voxels for each cluster are given. 


\section{Chapter 2}

Table S5. Significant decrements in functional connectivity of left nucleus accumbens, relative to placebo in the group receiving the full THC dose

\begin{tabular}{|c|c|c|c|c|c|c|c|}
\hline Cluster & $\mathrm{R}$ or $\mathrm{L}$ & BA & k & $x$ & y & $z$ & $\begin{array}{l}P \text { value } \\
t_{3.28} F W E \\
\text { cluster } \\
\text { corrected }\end{array}$ \\
\hline Angular gyrus & $\mathrm{R}$ & 7 & 33 & 32 & -58 & 54 & 0.001 \\
\hline Precuneus & L & 7 & 62 & -4 & -76 & 44 & 0.000 \\
\hline Precuneus & L & & 95 & -4 & -46 & 50 & 0.000 \\
\hline Precuneus & L & 5 & 27 & -6 & -48 & 72 & 0.005 \\
\hline Precuneus & L & 5 & 24 & -10 & -58 & 68 & 0.011 \\
\hline Middle temporal gyrus & L & 21 & 39 & -66 & -30 & 4 & 0.000 \\
\hline Middle temporal gyrus & L & 21 & 30 & -54 & -54 & 8 & 0.002 \\
\hline Superior temporal gyrus & R & 21 & 60 & 62 & -22 & 2 & 0.000 \\
\hline Inferior frontal gyrus, pars opercularis & $\mathrm{L}$ & 48 & 177 & -36 & 18 & 18 & 0.000 \\
\hline Insula & L & 48 & 40 & -34 & 10 & 2 & 0.000 \\
\hline Superior temporal gyrus & $\mathrm{R}$ & 48 & 71 & 50 & -28 & 12 & 0.000 \\
\hline Rolandic operculum & $\mathrm{R}$ & 48 & 145 & 62 & 0 & 12 & 0.000 \\
\hline Inferior frontal gyrus, pars opercularis & $\mathrm{R}$ & 48 & 30 & 50 & 18 & 18 & 0.002 \\
\hline Postcentral gyrus & L & 48 & 58 & -60 & 2 & 16 & 0.000 \\
\hline \multirow[t]{2}{*}{ Transverse temporal gyrus } & $\mathrm{R}$ & 48 & 21 & 38 & -20 & 12 & 0.029 \\
\hline & L & 48 & 22 & -30 & -30 & 20 & 0.021 \\
\hline Postcentral gyrus & L & 3 & 72 & -44 & -14 & 40 & 0.000 \\
\hline Precentral gyrus & $\mathrm{R}$ & 6 & 52 & 20 & -26 & 68 & 0.000 \\
\hline Precentral gyrus & $\mathrm{R}$ & 6 & 62 & 46 & 2 & 38 & 0.000 \\
\hline Precentral gyrus & $\mathrm{R}$ & 6 & 29 & 44 & -10 & 48 & 0.003 \\
\hline Middle frontal gyrus & L & 6 & 37 & -32 & -2 & 54 & 0.000 \\
\hline Supplementary motor area & L & 6 & 35 & -18 & 0 & 68 & 0.000 \\
\hline Supplementary motor area & $\mathrm{R}$ & 6 & 30 & 6 & 4 & 56 & 0.002 \\
\hline Superior occipital gyrus & L & 18 & 32 & -20 & -90 & 30 & 0.001 \\
\hline
\end{tabular}


Effects of cannabis on striatal glutamate and corticostriatal connectivity

\begin{tabular}{|c|c|c|c|c|c|c|c|}
\hline Lingual gyrus & L & 18 & 68 & -32 & -90 & -14 & 0.000 \\
\hline Lingual gyrus & L & 19 & 77 & -26 & -68 & -4 & 0.000 \\
\hline Fusiform gyrus & L & 19 & 45 & -38 & -72 & -14 & 0.000 \\
\hline Middle temporal gyrus & L & 20 & 37 & -50 & -10 & -14 & 0.000 \\
\hline Fusiform gyrus & $\mathrm{R}$ & 37 & 25 & 34 & -36 & -12 & 0.008 \\
\hline Fusiform gyrus & $\mathrm{R}$ & 37 & 31 & 38 & -60 & -10 & 0.001 \\
\hline Paracentral Lobule & $\mathrm{L}$ & 4 & 31 & -2 & -26 & 64 & 0.001 \\
\hline Inferior parietal gyrus & L & 40 & 28 & -46 & -46 & 44 & 0.003 \\
\hline Midcingulate area & L & & 30 & -6 & -20 & 44 & 0.002 \\
\hline Midcingulate area & $\mathrm{R}$ & & 21 & 10 & -18 & 44 & 0.029 \\
\hline
\end{tabular}

Statistical threshold: $\mathrm{P}<0.001$ (uncorrected). MNI coordinates of peak voxels for each cluster are given. 


\section{Chapter 2}

Table S6. Significant correlations between functional connectivity and absolute average change scores of the variables of interest.

\begin{tabular}{|c|c|c|c|c|c|c|c|c|c|c|}
\hline Correlation & $+/-$ & & Ro & & k & $\mathrm{x}$ & y & $z$ & $\begin{array}{l}\text { P value } t_{5.21} \\
\text { FWE cluster } \\
\text { corrected }\end{array}$ & Pearson's R \\
\hline & & Cluster & & BA & & & & & & \\
\hline \multirow[t]{13}{*}{$\mathrm{Glu} / \mathrm{tCr}$} & \multirow[t]{13}{*}{+} & & $\mathrm{L}$ & & \multirow{2}{*}{26} & \multirow{2}{*}{-46} & \multirow{2}{*}{-6} & \multirow{2}{*}{58} & \multirow{2}{*}{.000} & $r=.989, P$ \\
\hline & & Precentral gyrus & & 6 & & & & & & $=.000$ \\
\hline & & $\begin{array}{l}\text { Supplementary motor } \\
\text { area }\end{array}$ & L & 6 & 15 & -6 & -12 & 64 & .004 & $\begin{array}{l}r=.968, P \\
=.000\end{array}$ \\
\hline & & & $\mathrm{R}$ & & \multirow{2}{*}{18} & \multirow{2}{*}{42} & \multirow{2}{*}{-4} & \multirow{2}{*}{56} & \multirow{2}{*}{.001} & $r=.979$ \\
\hline & & Middle frontal gyrus & & 6 & & & & & & $P=.000$ \\
\hline & & & $\mathrm{L}$ & & \multirow{2}{*}{19} & \multirow{2}{*}{-2} & \multirow{2}{*}{60} & \multirow{2}{*}{20} & \multirow{2}{*}{.001} & $r=.969, P$ \\
\hline & & Medial frontal gyrus & & 10 & & & & & & $=.000$ \\
\hline & & & L & & \multirow{2}{*}{13} & \multirow{2}{*}{-12} & \multirow{2}{*}{44} & \multirow{2}{*}{14} & \multirow{2}{*}{.012} & $r=.976, P$ \\
\hline & & Anterior cingulate & & 32 & & & & & & $=.000$ \\
\hline & & & $\mathrm{L}$ & & \multirow{2}{*}{12} & \multirow{2}{*}{-40} & \multirow{2}{*}{26} & \multirow{2}{*}{-6} & \multirow{2}{*}{.021} & $r=.989, P$ \\
\hline & & Inferior frontal gyrus & & 47 & & & & & & $=.000$ \\
\hline & & & $\mathrm{L}$ & & \multirow{2}{*}{14} & \multirow{2}{*}{-58} & 2 & 10 & 007 & $r=.976, P$ \\
\hline & & Rolandic operculum & & 48 & & & 2 & 10 & . & $=.000$ \\
\hline VAS & - & & $\mathrm{L}$ & & 12 & -14 & -56 & 24 & .022 & $r=-.978, P=$ \\
\hline & & cumeus & & & & & & & & .000 \\
\hline Number of lapses & + & & $\mathrm{L}$ & & 15 & -16 & 68 & 8 & .004 & $r=.857, P=$ \\
\hline & & Superior frontal gyrus & & 10 & & & & & & .006 \\
\hline
\end{tabular}

MNI coordinates of peak voxels for each cluster are given. 


\section{Effects of cannabis on striatal glutamate and corticostriatal connectivity}

\section{References}

1 Frahm, J. et al. Localized high-resolution proton NMR spectroscopy using stimulated echoes: initial applications to human brain in vivo. Magnetic resonance in medicine 9, 79-93 (1989).

2 Tkac, I., Starcuk, Z., Choi, I. Y. \& Gruetter, R. In vivo 1 H NMR spectroscopy of rat brain at 1 ms echo time. Magnetic resonance in medicine 41, 649-656 (1999).

$3 \quad$ Klose, U. In vivo proton spectroscopy in presence of eddy currents. Magnetic resonance in medicine 14, 26-30 (1990).

$4 \quad$ Barker, P. B. et al. Quantitation of proton NMR spectra of the human brain using tissue water as an internal concentration reference. NMR in biomedicine 6, 89-94 (1993).

5 Soher, B. J., Hurd, R. E., Sailasuta, N. \& Barker, P. B. Quantitation of automated single-voxel proton MRS using cerebral water as an internal reference. Magnetic resonance in medicine 36, 335-339 (1996).

6 Smith, S. A., Levante, T. O., Meier, B. H. \& Ernst, R. R. Computer Simulations in Magnetic Resonance. An Object-Oriented Programming Approach. Journal of Magnetic Resonance 106, 75-105 (1994).

7 Govindaraju, V., Young, K. \& Maudsley, A. A. Proton NMR chemical shifts and coupling constants for brain metabolites. NMR in biomedicine 13, 129-153 (2000).

8 Penner, J. \& Bartha, R. Semi-LASER 1 H MR spectroscopy at 7 Tesla in human brain: Metabolite quantification incorporating subject-specific macromolecule removal. Magnetic resonance in medicine, doi:10.1002/mrm.25380 (2014).

9 Marques, J. P. et al. MP2RAGE, a self bias-field corrected sequence for improved segmentation and T1-mapping at high field. Neurolmage 49, 1271-1281, doi:10.1016/j.neuroimage.2009.10.002 (2010).

10 Zhang, Y., Brady, M. \& Smith, S. Segmentation of brain MR images through a hidden Markov random field model and the expectation-maximization algorithm. IEEE transactions on medical imaging 20, 45-57, doi:10.1109/42.906424 (2001).

11 Puts, N. A. J. \& Edden, R. A. E. In vivo magnetic resonance spectroscopy of GABA: a methodological review. Progress in nuclear magnetic resonance spectroscopy 60, 29-41, doi:10.1016/j.pnmrs.2011.06.001 (2012).

12 Shungu, D. C. et al. Brain $\gamma$-aminobutyric acid (GABA) detection in vivo with the J-editing (1) H MRS technique: a comprehensive methodological evaluation of sensitivity enhancement, macromolecule contamination and test-retest reliability. NMR in biomedicine 29, 932-942, doi:10.1002/nbm.3539 (2016).

13 Loh, S., Lamond, N., Dorrian, J., Roach, G. \& Dawson, D. The validity of psychomotor vigilance tasks of less than 10-minute duration. Behavior research methods, instruments, \& computers : a journal of the Psychonomic Society, Inc 36, 339-346 (2004). 


\section{Chapter 3}

\section{Reduced responsiveness of the reward system is associated with tolerance to cannabis impairment in chronic users}

Mason, N.L., Theunissen, E.L., Hutten, N.R.P.W., Tse, D.H.Y., Toennes, Jansen, J.F.A., S.W., Stiers, P., and Ramaekers, J.G.

Cannabis is the most commonly used illicit drug in the world. However, because of a changing legal landscape and rising interest in therapeutic utility, there is an increasing trend in (long-term) use and possibly cannabis impairment. Importantly, a growing body of evidence suggests that regular cannabis users develop tolerance to the impairing, as well as the rewarding, effects of the drug. However, the neuroadaptations that may underlie cannabis tolerance remain unclear. Therefore, this double-blind, randomized, placebo-controlled, cross-over study assessed the acute influence of cannabis on the brain and behavioral outcomes in two distinct cannabis user groups. Twelve occasional and 12 chronic cannabis users received acute doses of cannabis (300- $\mu \mathrm{g} / \mathrm{kg}$ delta-9tetrahydrocannabinol) and placebo and underwent ultrahigh field functional magnetic resonance imaging and magnetic resonance spectroscopy. In occasional users, cannabis induced significant neurometabolic alterations in reward circuitry, namely, decrements in functional connectivity and increments in striatal glutamate concentrations, which were associated with increases in subjective high and decreases in performance on a sustained attention task. Such changes were absent in chronic users. The finding that cannabis altered circuitry and distorted behavior in occasional, but not chronic users, suggests reduced responsiveness of the reward circuitry to cannabis intoxication in chronic users. Taken together, the results suggest a pharmacodynamic mechanism for the development of tolerance to cannabis impairment, of which is important to understand in the context of the long-term therapeutic use of cannabis-based medications, as well as in the context of public health and safety of cannabis use when performing day-to-day operations.

Addiction Biology, 2019 


\section{Chapter 3}

\section{Introduction}

Cannabis is the most commonly used illicit drug in the world, with $4 \%$ of the global population reportedly using $\mathrm{it}^{1}$. However due to a changing legal landscape, and rising interest in therapeutic utility, there is an increasing trend in (long-term) use $\mathrm{i}^{1,2}$. Importantly, a growing body of evidence suggests that the acute effects of cannabis are less prominent in regular cannabis users ${ }^{3}$, suggesting development of tolerance to the impairing, as well as the rewarding, effects of the drug. Nonetheless, the neurobiological mechanisms underlying cannabis tolerance are unknown.

Accumulating evidence suggests that the main psychoactive component of cannabis [delta-9-tetrahydrocannabinol (THC)] binds to cannabinoid (CB1) receptors located on GABAergic and glutamatergic neurons distributed throughout the brain, with high densities found in limbicreward structures ${ }^{4}$. Subsequently, THC has been found to acutely activate the reward circuitry, increasing dopamine ${ }^{5-9}$ and glutamate ${ }^{10,11}$ concentration levels in key brain areas including the striatum, nucleus accumbens (NAc) and prefrontal cortex (PFC); a pattern implicated in both the rewarding and impairing effects of drugs of abuse $\mathrm{e}^{12,13}$.

Accordingly, studies with chronic cannabis users have found alterations in dopaminergic function in striatal areas ${ }^{14-16}$, as well as decreases in glutamate concentrations in the basal ganglia 17,18 and anterior cingulate cortex $(A C C)^{19,20}$. Furthermore, repeated use of cannabis has been associated with structural changes in frontal areas, as evinced by decreased grey matter volume ${ }^{21-}$

23 , and abnormal concentrations of metabolites including N-acetylaspartate (NAA), myo-inositol $(\mathrm{ml})$, and choline containing compounds (Cho), biochemical markers of neuronal integrity and glial activation 18,19,24-28. Taken together, these studies provide evidence that repeated cannabis exposure may lead to alterations in neurotransmission and neuronal health, which underlie the diminished cognitive and behavioral response associated with acute cannabis tolerance. However, to date the neuroadaptations that may underlie cannabis tolerance have not been systematically assessed.

Therefore, the aim of the present double-blind, placebo controlled study was twofold. The first goal was to assess acute influence of cannabis (300 $\mathrm{gg} / \mathrm{kg} \mathrm{THC}$ ) in two different cannabis using groups, namely occasional and chronic users, on brain and behavioral outcomes previously found to be affected by cannabis. Ultra-High Field (7T) proton magnetic resonance spectroscopy (MRS) was used to assess glutamate, GABA, NAA, Cho, and $\mathrm{ml}$ levels in the striatum and ACC. Resting state functional magnetic resonance imaging ( $F M R I$ ) data were acquired to determine functional connectivity (FC) between the regions of interest (ROI) in the NAc and remote cortical areas, as an indirect measure of dopaminergic stimulation ${ }^{10,29,30}$. Furthermore a priori ROI-to-ROI analysis assessed differences in connectivity strength between areas of the reward circuit. Finally, 
subjective high and sustained attention, two outcome variables shown to be modulated by (acute) cannabis exposure ${ }^{10,31,32}$, were assessed.

The second goal was exploratory, to evaluate long-term effects of repeated cannabis exposure, by comparing the placebo condition of each group. Overall, we hypothesized that THC would induce behavioral, functional, and metabolic changes in occasional, but not chronic users, indicative of (neuroadaptive) tolerance. Furthermore, based on previous studies with chronic users, we hypothesized that during placebo, chronic users would show decreased concentrations of metabolites compared to occasional users.

\section{Materials and Methods}

A detailed description of the methodology is provided in the Supplementary, and briefly summarized here.

The study was conducted according to a double-blind, placebo-controlled, mixed crossover design. Twelve occasional and 12 chronic users (male $\mathrm{N}=14$ ) received placebo and $300 \mu \mathrm{g} / \mathrm{kg}$ THC (Bedrobinol; 13.5 \% THC) on separate days, separated by a minimum wash-out period of 7 days for occasional users. Sample size was based off of power calculations and previous studies assessing reward system activation during acute THC intoxication ${ }^{10,30}$

This study was conducted according to the code of ethics on human experimentation established by the declaration of Helsinki (1964) and amended in Fortaleza (Brazil, October 2013) and in accordance with the Medical Research Involving Human Subjects Act (WMO) and was approved by the Academic Hospital and University's Medical Ethics committee. All participants were fully informed of all procedures, possible adverse reactions, legal rights and responsibilities, expected benefits, and their right for voluntary termination without consequences. All participants gave their informed consent, in writing.

Image acquisition. All participants underwent a functional MRI and single-voxel MRS. Images were acquired on a MAGNETOM 7T MR scanner.

Spectroscopic voxels were placed in the ACC (voxel size $=25 \times 20 \times 17 \mathrm{~mm}^{3}$ ) and the right striatum (voxel size $=20 \times 20 \times 20 \mathrm{~mm}^{3}$ ). Spectra were acquired with stimulated echo acquisition mode $(\mathrm{STEAM}){ }^{33}$ sequence $(\mathrm{TE}=6.0 \mathrm{~ms}, \mathrm{TR}=5.0 \mathrm{~s}, 64$ averages). Outcome measures for MRS were concentration ratios of glutamate, GABA, NAA, $\mathrm{ml}$, and Cho to total Creatine ( $\mathrm{tCr}$, Creatine + Phospho-Creatine).

Additionally, 258 whole-brain EPI volumes were acquired at rest (TR=1400 ms; TE= 21 ms; flip angle $=60^{\circ}$; oblique acquisition orientation; interleaved slice acquisition; 72 slices; slice thickness $=1.5 \mathrm{~mm}$; voxel size $=1.5 \times 1.5 \times 1.5 \mathrm{~mm}$ ). During scanning, participants were shown a black 
cross on a white background, and were instructed to focus on the cross while attempting to clear their mind.

Processing of imaging data. Spectroscopy data was analyzed with LCModel version $6.3-1 \mathrm{H}$.

FC data were produced with the MATLAB toolbox DPARSF ${ }^{34}$. In order to indirectly assess dopamine neurotransmission, two spheres ( $4 \mathrm{~mm}$ radius) were created that were located (in MNI space) in the left and right NAc. Average time courses were obtained for each sphere separately and correlational analysis was performed voxel wise to generate FC maps for each sphere.

Furthermore, as we were interested in FC within the reward circuit, ROI-to-ROI FC was computed according to the same aforementioned procedure, between areas including: NAc, medial dorsal nucleus (MDN), ventral pallidum (VP), and midcingulate area (MC). Fisher's correlation coefficient maps were created between the NAc and MDN, NAc and VP, MDN and VP, MDN and MC, and MC and NAC.

Subjective and Behavioral Measures. Sustained attention was assessed via the psychomotor vigilance task (PVT), a reaction-time task that measures the speed with which participant respond to a visual stimulus ${ }^{35}$. The outcome measures of the task are response speed (mean reaction time) and number of attentional lapses (reaction time $>500 \mathrm{~ms}$ ).

Participants also rated their subjective high on visual analogue scales $(10 \mathrm{~cm})$ on four consecutive time points after treatment administration, on a scale between 0 (not high at all) to 10 (extremely high).

Pharmacokinetic Measures. Blood samples $(8 \mathrm{~mL}$ ) to determine cannabinoid concentrations (THC and metabolites $\mathrm{OH}-\mathrm{THC}$ and $\mathrm{THC}-\mathrm{COOH}$ ) were taken at base- line, 10, 30, 50, and 70 minutes post administration, and analyzed according to a standardized procedure ${ }^{10}$.

\section{Statistical Analysis}

Subjective high, sustained attention, and metabolite concentrations. A mixed model analysis was performed consisting of the within-subject factors treatment (THC and placebo), and time after smoking (2 levels), and the between-subject factor of group (occasional or chronic) (SPSS Version 24; SPSS Inc., Chicago, IL). Due to main effect of Treatment or interaction of Treatment X Group, a second analysis was performed for each group, with treatment and time as within-subject factors. The alpha criterion level of significance was set at $p=0.05$. Due to a violation of the assumption of normality, the data for the number of lapses and mean reaction time were log transformed. 
fMRI data. FC data (i.e. correlation coefficient maps for each individual in each treatment condition at each time point) were analyzed in a GLM model in SPM 12.

For the reward circuit ROI-to-ROI analysis of Fisher's correlation coefficient values was conducted in IBM SPSS statistics 24. For each group, a repeated measures analysis was conducted consisting of the within-subject factor treatment (THC and placebo).

Correlation analysis. Correlation analyses were conducted to further investigate the relationship between cannabis induced changes in brain and behavior. Correlation input included average treatment change values of (i) behavioral outcomes (subjective high and number of lapses), (ii) MRS concentration levels, (iii) ROI-to-ROI Fisher's correlation values, and (iv) mean voxel activation of SPM identified clusters from a voxel wise correlation analysis between NAc FC and behavioral outcomes. Pearson's correlations were performed in SPSS.

Exploratory analysis. In order to assess long-term effects of repeated cannabis exposure, separate analyses of covariance (ANCOVAS) were carried out for average (placebo) neurometabolite concentrations, behavioral measures, and FC in the reward circuit, as the dependent variables and group (occasional vs chronic user) as the fixed factor. Serum THC, 11-OH-THC, and THC-COOH levels were entered as covariates, due to significant differences between groups.

\section{Results}

Demographic Characteristics. occasional users $(n=12)$ and chronic users $(n=12)$ did not differ with respect to gender distribution, age, history of cannabis use, or consumption of alcohol, caffeine, nicotine, or other drugs (Table S1). As expected, chronic users reported using significantly more cannabis per week than occasional users.

THC Concentrations in Serum. Mean (SE) concentrations of THC, 11-OH-THC, and THC-COOH in serum are given in Table S2. As expected from previous experience ${ }^{36}$, chronic users not only exhibited significantly higher THC-COOH concentrations, but also reached significantly higher THC levels from the same dose regimen than occasional users.

Subjective and Cognitive Effects. Mixed model ANOVA [Treatment(THC vs placebo) *Time(timepoint 1 vs timepoint 2)*Group(occasionalvs chronic users)] yielded a significant main effect of Treatment $\left[F(1,21)=49.682, P<.0001, \eta p^{2}=.703\right]$ and Time $[F(3,63)=42.269, P<.0001$, $\left.\eta \mathrm{p}^{2}=.668\right]$, and a significant interaction of Treatment*Group $\left[\mathrm{F}(1,21)=5.023, \mathrm{P}=.036, \eta \mathrm{p}^{2}=.193\right]$ and Treatment*Time $\left[F(363)=17.346, \mathrm{P}<.0001, \eta \mathrm{p}^{2}=.452\right]$ on subjective high, indicating that $\mathrm{THC}$ increased feelings of intoxication in both groups, but to a larger degree in occasional users (Figure S1A). 
Analysis yielded a significant main effect of Treatment $\left[F(1,20)=8.057, P=.010, n p^{2}=.287\right]$ and Time $\left[F(1,20)=7.620, P=.012, n p^{2}=.276\right]$, and a significant interaction of Time* Group $[F(1,20)$ $=8.085, \mathrm{P}=.010, n \mathrm{p}^{2}=.288$ ] on mean reaction time of the PVT. Further analysis revealed that mean reaction time was significantly increased by $T H C$ in occasional users $[F(1,10)=5.226, P=.045$, $\left.\eta \mathrm{p}^{2}=.343\right]$, but not in chronic users [P>.1] (Figure S1B).

Analysis yielded a significant main effect of Treatment $\left[F(1,21)=7.793, P=.011, \eta p^{2}=.271\right]$, and a significant interaction of Time* $\operatorname{Group}\left[F(1,21)=4.313, P=.050, n p^{2}=.170\right]$ on number of attentional lapses during the PVT. Further analysis revealed number of attentional lapses was significantly increased by THC in occasional users $\left[F(1,10)=5.286, P=.044, n p^{2}=.346\right]$, but not in chronic users [P>.1] (Figure S1C).

\section{Metabolite Concentrations.}

Striatum. Mixed model ANOVA yielded a significant interaction of Treatment *Time *Group $\left[F(1,21)=8.779, P=.007, n p^{2}=.295\right]$ on glutamate $/ \mathrm{tCr}(\mathrm{Glu})$ concentration levels. Further analysis revealed that concentration levels were significantly increased by THC in occasional users $[F(1,11)$ $\left.=11.506, P=.006, \eta p^{2}=.511\right]$, but not in chronic users $[P>.1]$ (Figure $\left.1 A\right)$.

Analysis yielded a significant interaction of Treatment *Time *Group $[F(1,19)=6.546$, $\left.P=.019, n p^{2}=.256\right]$ and a significant main effect of Treatment $\left[F(1,19)=4.670, P=.044, n p^{2}=.197\right]$ on NAA + NAAG/tCr (NAA) concentration levels. Further analysis revealed that concentration levels were significantly increased by THC in occasional users $\left[F(1,11)=15.345, P=.002, n p^{2}=.582\right]$, but not in chronic users $[P>$.1] (Figure $1 B)$.

Analysis yielded a trending interaction of Treatment*Group $[F(1,20)=4.241, P=.053$, $\left.\eta \mathrm{p}^{2}=.175\right]$ on $\mathrm{ml} / \mathrm{tCr}(\mathrm{ml})$ concentration levels. Further analysis revealed that concentration levels were significantly increased by THC in occasional users $\left[F(1,11)=5.176, P=.044, n p^{2}=.320\right]$, but not in the chronic users $[P>.1]$ (Figure $1 C$ ).

Anterior cingulate cortex. Mixed model ANOVA yielded a significant interaction of Treatment*Group $\left[F(1,18)=7.450, P=.014, n p^{2}=.293\right]$ on $\mathrm{ml}$ concentration levels. Further analysis revealed that concentration levels were significantly decreased by THC in chronic users $[F(1,9)=$ 5.935, $\left.P=.038, n p^{2}=.397\right]$, but not in occasional users [P>.1] (Figure 1D)].

Other metabolites did not reach significance (see Table S3 for mean metabolite concentrations). 

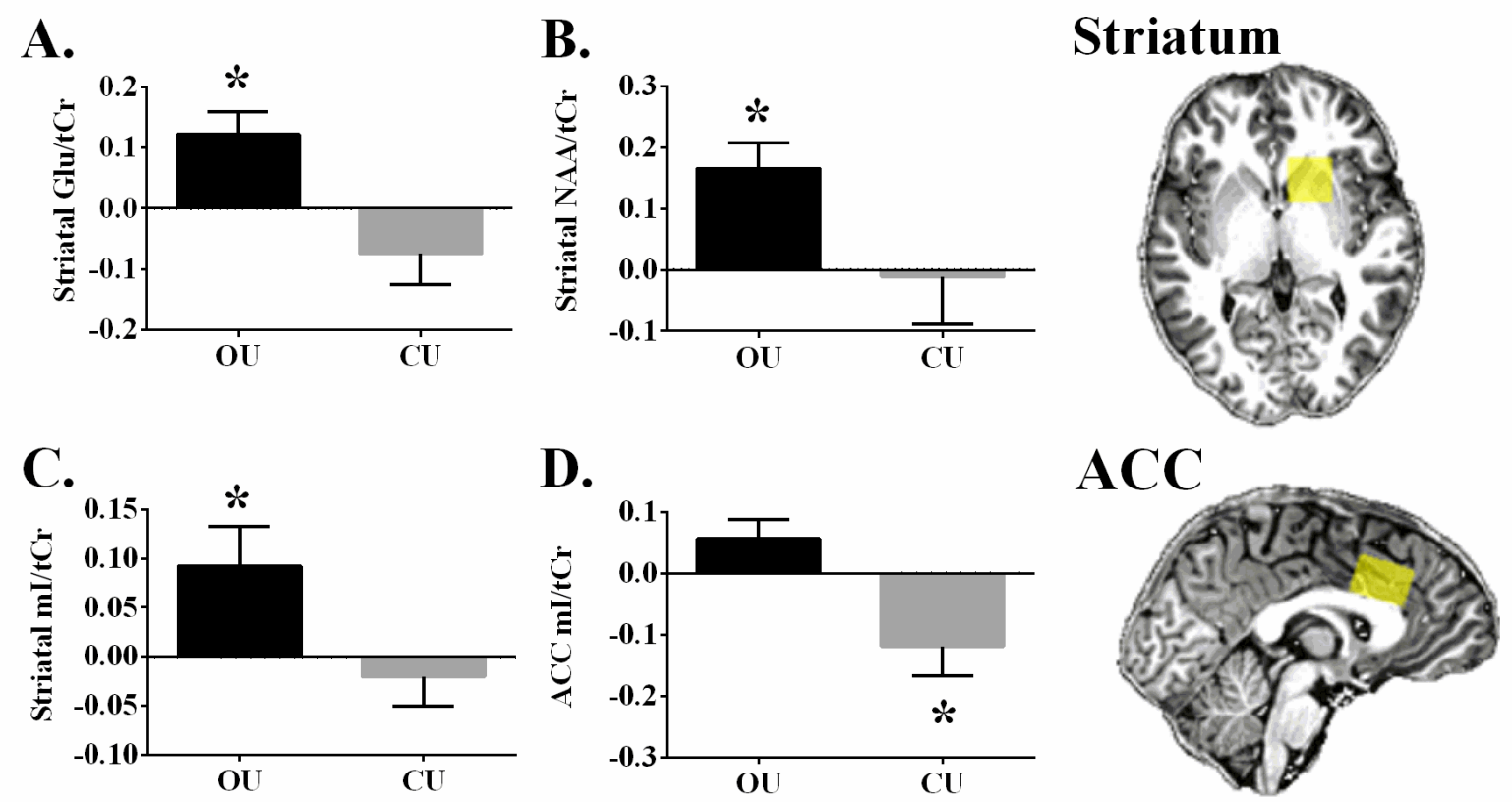

Figure 1. Occasional and chronic users mean (SE) metabolite concentration levels, averaged over both timepoints for both treatments [average(THC timepoint 1 - Placebo timepoint 2; THC timepoint 2 - Placebo timepoint 2)] A. Striatal glutamate. B. Striatal total $n$-acetyl-aspartate. C. Striatal myoinositol. D. Anterior cingulate cortex myoinositol. OU=occasional user; $C U=c h r o n i c$ user; * within group analysis, $P<.05$

Functional Connectivity with the Nucleus Accumbens. The contrast drug vs placebo (Placebo > THC) resulted in reduced FC with the NAc seeds in both hemispheres in occasional users, whereas no change was found in chronic users (Figure 2). Reductions in FC were prominent in broad areas of the frontal, temporal, parietal, and occipital lobes, a pattern typical of an increase in dopaminergic neurotransmission (Table S4). No significant differences in activation were found for the inverse comparison (THC > Placebo) in either group. Furthermore, no significant differences were seen between the left and right NAc seed, so only left NAc seed results are shown. 


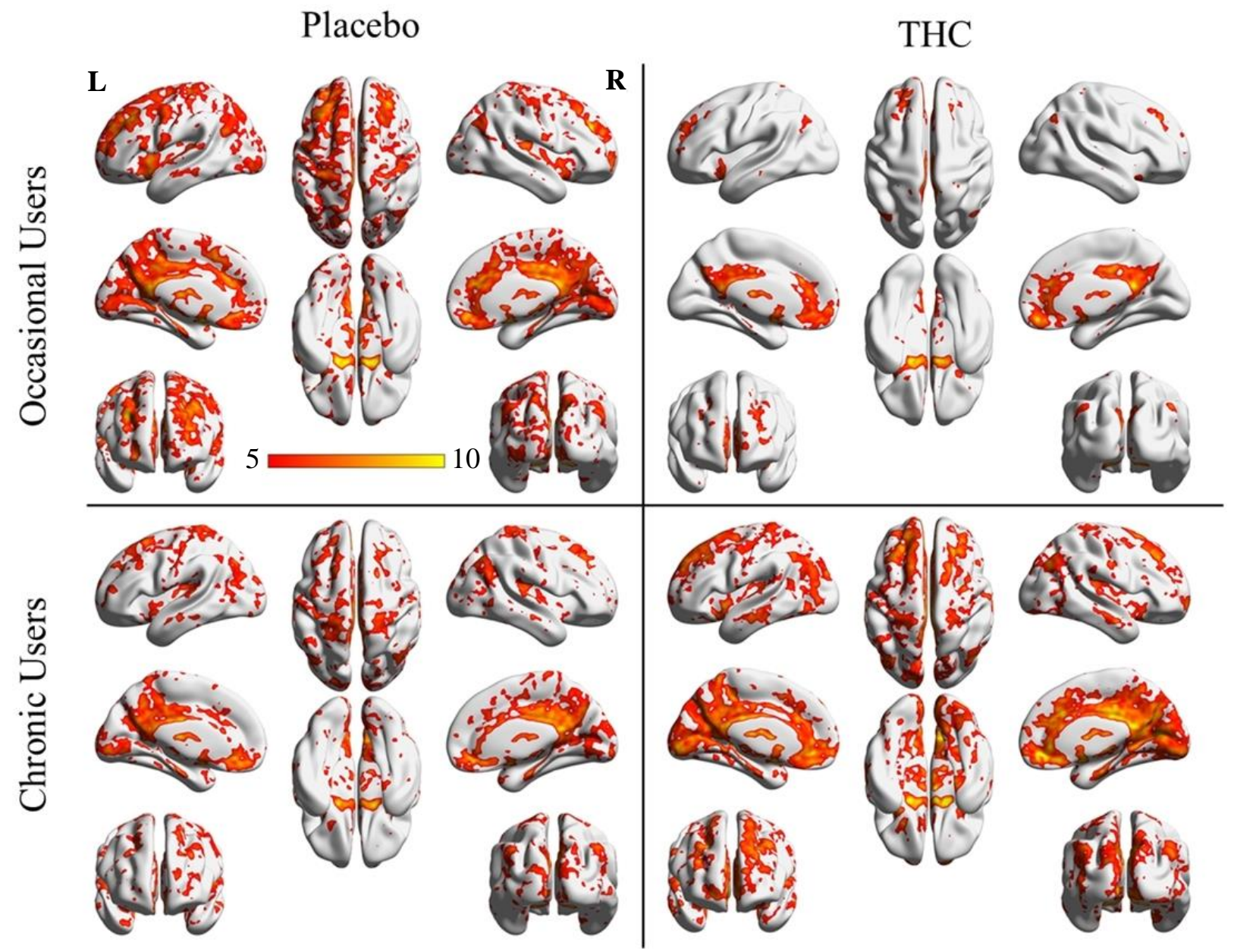

Figure 2. NAcc-related functional connectivity in the left hemispheres. Shown are thresholded Z-score maps of functional connectivity for each group, and each condition.

Functional Connectivity in the Reward Circuit. Results of the region-of-interest (ROI)-to-ROI FC analysis are displayed in (Figure 3). ROls were chosen because they are established structures of the reward circuitry, a cortico-subcortical network connected via glutamatergic and GABAergic projections between the NAc, VP, MDN, and the PFC ${ }^{37-40}$ (Figure 3). NAc, VP, and MDN seeds were chosen a priori, whereas the prefrontal seed was chosen based on the previous FC analysis, which indicated significant treatment (Placebo > THC) induced changes in the MC. Mixed model ANOVA revealed that cannabis decreased FC between the ROIs in occasional users, whereas no significant treatment effect was seen in chronic users. 
A.

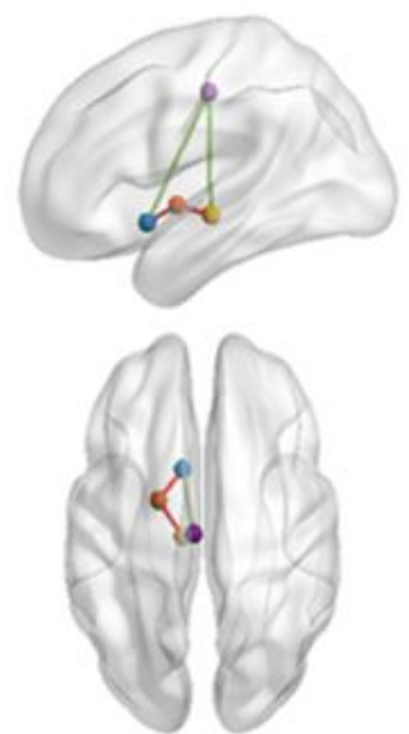

NAc V VP

$\mathrm{MDN}$ 므

B.

\begin{tabular}{|c|c|c|c|c|c|c|}
\hline Group & Connection & Effect & Df & $\mathrm{F}$ & $P$ & $\eta_{p}^{2}$ \\
\hline \multirow[t]{5}{*}{$\mathrm{OU}$} & $\mathrm{Nac} \leftarrow \rightarrow \mathrm{VP}$ & THC $\downarrow$ & 1,11 & 4.38 & .06 & .28 \\
\hline & $\mathrm{Nac} \leftarrow \rightarrow \mathrm{MDN}$ & THC $\downarrow$ & 1,11 & 7.07 & .02 & .39 \\
\hline & $\mathrm{VP} \leftarrow \rightarrow \mathrm{MDN}$ & THC $\downarrow$ & 1,11 & 3.95 & .07 & .26 \\
\hline & $\mathrm{MC} \leftrightarrow \mathrm{MDN}$ & THC $\downarrow$ & 1,11 & 18.13 & .001 & .62 \\
\hline & $\mathrm{MC} \leftarrow \rightarrow \mathrm{Nac}$ & THC $\downarrow$ & 1,11 & 25.73 & .000 & .70 \\
\hline \multirow[t]{5}{*}{$\mathrm{CU}$} & $\mathrm{Nac} \leftarrow \rightarrow \mathrm{VP}$ & - & 1,10 & .01 & .91 & .001 \\
\hline & $\mathrm{Nac} \leftarrow \rightarrow \mathrm{MDN}$ & - & 1,10 & .30 & .59 & .03 \\
\hline & $\mathrm{VP} \leftarrow \rightarrow \mathrm{MDN}$ & - & 1,10 & .45 & .61 & .06 \\
\hline & $\mathrm{MC} \leftrightarrow \rightarrow \mathrm{MDN}$ & - & 1,10 & .04 & .85 & .00 \\
\hline & $\mathrm{MC} \leftarrow \rightarrow \mathrm{Nac}$ & - & 1,10 & 1.33 & .27 & .12 \\
\hline
\end{tabular}

Figure 3. ROI-to-ROI FC analysis results. A) Four nodes of the reward circuit, where ROls were placed. Red lines pertain to gabaergic pathways, whereas green lines pertain to glutamatergic pathways. NAc = nucleus accumbens; $V P=$ ventral pallidum; $M D N=$ medial dorsal nucleus; $M C=$ midcingulate area B) Results of repeated measures analysis (THC vs placebo), separated by group. $\mathrm{OU}=$ occasional user; $\mathrm{CU}=$ chronic user

Relationship Between Cannabis Induced Changes in Brain and Behavior. Analysis revealed a significant positive correlation in occasional users ( $r=.641)$ between treatment induced changes in striatal NAA and number of attentional lapses, and a significant negative correlation ( $r=-.618$ ) between treatment induced changes in striatal $\mathrm{ml}$ and subjective high. For an overview of all correlational analyses, see Table S7. A further voxel wise correlation analysis between NAc FC whole brain correlation coefficient maps and behavioral outcomes was performed, revealing significant positive correlations (all $r>.6$ ) in FC between the NAc and cortical brain areas, and behavioral outcomes (Table S8).

Exploratory Analysis. No significant difference between groups was found in any of the variables of interest, when controlling for THC and THC-metabolite concentrations in blood $(P>.1)$.

\section{Discussion}

The present study demonstrates the first attempt to assess cannabis induced neuroadaptations in the reward system, which may underlie behavioral cannabis tolerance. Using an ultra-high field multimodal brain imaging approach, we showed that in occasional users, cannabis induced significant neurometabolic alterations in the reward circuitry, namely decrements in FC and 
increments in striatal glutamate, which were associated with increases in subjective high and decreases in performance on a sustained attention task. Such changes were absent in chronic users. The finding that cannabis altered reward circuitry and distorted behavior in occasional, but not chronic users, suggests the development of neuroadaptations in the reward circuitry after excessive use of cannabis that reduce the circuitry and behavioral response to acute cannabis impairment.

The present study found that, in occasional users, cannabis decreased coupling between BOLD responses in keys areas of the reward system, a fronto-subcortical network of brain structures that are connected via dopaminergic, GABAergic, and glutamatergic neurotransmission (i.e. nucleus accumbens, VTA, ventral pallidum), motivation and salience attribution (i.e. medial orbital frontal cortex), executive and inhibitory control (i.e. anterior cingulate cortex) and conditioning and memory (i.e. amygdala, medial orbital frontal cortex, hippocampus) 41,42. Importantly, decrements in FC between the NAc and regions such as the thalamus and frontal cortex have been suggested to reflect increases in dopaminergic neurotransmission throughout the circuit 10,29,30. Specifically, the NAc receives dopaminergic input from the VTA, which is under inhibitory control of GABA interneurons on which presynaptic CB1 receptors are located. Stimulation of CB1 receptors by THC disinhibits the VTA, which in turn increases dopamine levels in the NAC ${ }^{43}$. Subsequently, the increase in striatal dopamine level output from the NAc decreases the GABAergic inhibitory tone to the thalamus, which is reflected in decreased FC, as seen in this study.

Furthermore, this process of disinhibition has been suggested to lead to increased glutamatergic signaling to the PFC, subsequently to the VTA, and back to the NAC ${ }^{30}$. Accordingly, MRS in occasional users showed cannabis increased striatal glutamate concentration levels; a finding that is compatible with previous human studies which have found acute increases ${ }^{10,11}$, as well as chronic decreases ${ }^{18}$ of striatal glutamate after exposure to THC. Furthermore, it has previously been demonstrated that cannabis induced changes in striatal glutamate levels correlate strongly to cannabis induced alterations of FC within the fronto-subcortical circuit ${ }^{10}$. Thus it could be hypothesized that stimulatory glutamatergic input from the PFC to the NAc synergizes with cannabis induced increases in dopaminergic input from the VTA to the NAc, and further strengthens the disinhibition of thalamic signaling in the fronto-subcortical circuitry. However, MRS did not show altered ACC glutamate concentrations in the OU group, as would be hypothesized. Indeed previous results regarding the effects of cannabis on glutamate in the ACC have been mixed, with studies by one group finding reductions of glutamate-related metabolite concentrations in cannabis users ${ }^{19,20}$, whereas no alterations were found in a subsequent chronic 28 , or acute ${ }^{10}$ study. Nonetheless, previous work utilizing microdialysis found THC increased extracellular glutamate in the rat $\mathrm{PFC}^{9}$, supporting the hypothesis that $\mathrm{THC}$ disrupts glutamatergic 
signaling in frontal areas, however this effect may not be localized to the ACC. Thus future studies should assess the impact THC has on other areas in the frontal cortex, like the medial orbitofrontal cortex.

The present study also demonstrated that cannabis increased feelings of subjective high and decreased performance on a sustained attention task in occasional users, outcome variables previously found to be affected by cannabis ${ }^{10,31,32}$. Furthermore, behavioral outcomes correlated with striatal FC to other areas of the reward circuit. Accordingly, cannabis induced changes in striatal glutamate and striatal FC have been significantly associated with decrements in cognitive function and impulse control ${ }^{30}$, as well as increases in subjective high ${ }^{10,30}$ and experience of psychotomimetic symptoms ${ }^{11}$. Taken together, the findings suggest that the impact of cannabis on neural activity within the reward circuit may underlie multiple behavioral changes observed after acute cannabis exposure.

In line with this, chronic users demonstrated an absence of cannabis induced stimulation of the reward circuit, as well as mitigation of behavioral alterations. Specifically, no changes were seen in either FC between areas of the reward circuitry, or glutamate concentration levels, when comparing cannabis to placebo. Furthermore, sustained attention performance did not significantly differ between treatment conditions. However, chronic users reported significantly increased levels of subjective high after cannabis relative to placebo, although the change in high was to a lesser extent than in the occasional users, as expected ${ }^{31}$. Taken together, findings suggest that chronic cannabis users exhibit pharmacodynamic tolerance to the effects of cannabis.

The mechanisms by which neurobiological tolerance to the acute effects of THC develops have yet to be fully elucidated. Animal and human research generally supports the notion of CB1 receptors downregulation and desensitization in cortical and subcortical regions after repeated exposure to cannabis ${ }^{44-51}$. Although studies have reported global reduction in CB1 receptor availability in chronic cannabis users, it has been suggested that neuroadaptive changes take place in a time and region-specific manner ${ }^{52}$, with regional analysis demonstrating significant CB1 receptor decrements in areas such as the ACC and NAC ${ }^{50}$. Thus, the absence of change in FC of the NAC with other parts of the reward circuit could suggest that downregulation of CB1 receptors mitigate the impact of acute cannabis intoxication on neural activity within fronto-subcortical circuits and associated behavioural outcomes, thus demonstrating the prime mechanisms underlying the development of tolerance in this circuit. However, to further explore the association between CB1 receptor availability and development of tolerance, future studies should assess CB1 receptor availability during acute intoxication.

In order to assess potential long-term effects of repeated cannabis exposure, analyses were performed comparing the placebo conditions between chronic and occasional users on ROI- 
to-ROI FC within the reward circuit, metabolite concentration levels, and performance on the sustained attention task. As chronic users had significantly higher baseline serum THC and THCmetabolite levels than occasional users, these values were added as covariates. When controlling for serum concentration levels, no differences were seen between groups on any of the outcome variables. Absence of group differences are in line with previous PET studies which have found CB1 receptor availability normalization in cannabis dependent users after as little as 2 days of monitored abstinence 49,51 , as well as neuropsychological data suggesting reversible cognitive deficits, modulated more by recent exposure than by cumulative lifetime use ${ }^{53}$. However, the literature on long-lasting effects of cannabis use is mixed, with studies also reporting long-term changes on brain structure ${ }^{22,23}$, neurometabolite levels ${ }^{54}$, and neurocognitive functioning ${ }^{55}$. Importantly, results have been found to be region and domain specific, and influenced by factors such as frequency and age of onset of use, potentially explaining the variance in reported outcomes. In order to further assess chronic effects of repeated cannabis exposure on the reward system and associated behaviour, future studies should employ a larger sample size and take such factors into account.

Finally, MRS demonstrated cannabis induced changes in neurometabolites previously found to be altered in cannabis users ${ }^{54}$, namely NAA and ml. NAA and ml, as well as glutamate, are markers of glial and neuronal activation ${ }^{56,57}$. Our data demonstrate that cannabis acutely increases these metabolites in the striatum in occasional users. Treatment induced changes in NAA and $\mathrm{ml}$ also correlated with behavioural changes. Specifically, it was found that cannabis induced changes in $\mathrm{ml}$ in the striatum negatively correlated with cannabis induced changes in subjective high. Interestingly, elevations in $\mathrm{ml}$ and glutamate have been found in individuals with first-episode psychosis, and were found to correlate with subjective reports of grandiosity ${ }^{58}$. Furthermore, in the present study it was found that cannabis induced changes in NAA positively correlated with cannabis induced changes in sustained attention performance. Similarly, altered levels of NAA and NAAG have been reported in patients with schizophrenia, albeit in the ACC, with NAA levels correlating with attention performance ${ }^{59}$. Taken together, results suggest that cannabis increases glial and neuronal activation in the striatum, which is associated with changes in subjective state and behavioural performance. Importantly, these changes were not seen in the chronic users, further demonstrating pharmacodynamic tolerance. However, cannabis decreased $\mathrm{ml}$ in the ACC in chronic, but not occasional, users. The finding of acute decrease in ACC $\mathrm{ml}$ is compatible with previous studies which found decreased $\mathrm{ml}$ in the ACC ${ }^{19,20}$, as well as throughout the brain ${ }^{26,27}$, and has been suggested to reflect cannabis-related immunosuppression ${ }^{54}$.

In summary, our study provides previously unidentified evidence to suggest that a reduced responsiveness of the reward circuitry underlies a blunted pharmacodynamic response to an acute cannabis challenge in chronic users. Understanding the neuroadaptive basis of tolerance is 
important in the context of the therapeutic use of cannabis-based medications, as well as in the context of public health and safety of cannabis use when performing day-to-day operations.

\section{Funding and Disclosure}

The authors declare no conflict of interest.

\section{Acknowledgements}

The authors would like to thank Cees van Leeuwen and Lizzy Vuurman for medical supervision, and all participants for their time and effort 


\section{Chapter 3}

\section{References}

$1 \quad$ UN. World Drug Report 2018. United Nations publication (2018).

2 EMCDDA. European Drug Report 2018: Trends and Developments. Publications Office of the European Union, Luxemborg, doi:10.2810/800331 (2018).

3 Colizzi, M. \& Bhattacharyya, S. Cannabis use and the development of tolerance: a systematic review of human evidence. Neurosci Biobehav Rev 93, 1-25, doi:10.1016/j.neubiorev.2018.07.014 (2018).

$4 \quad$ Iversen, L. Cannabis and the brain. Brain : a journal of neurology 126, 1252-1270 (2003).

5 Diana, M., Melis, M. \& Gessa, G. Increase in meso-prefrontal dopaminergic activity after stimulation of CB1 receptors by cannabinoids. Eur J Neurosci 10, 2825-2830 (1998).

$6 \quad$ Fadda, P. et al. Cannabinoid self-administration increases dopamine release in the nucleus accumbens. Neuroreport 17, 1629-1632 (2006).

7 Ton, J. M. N. C. et al. The effects of $\Delta 9$-tetrahydrocannabinol on potassium-evoked release of dopamine in the rat caudate nucleus: an in vivo electrochemical and in vivo microdialysis study. Brain research 451, 59-68 (1988).

8 Bossong, M. G. et al. Further human evidence for striatal dopamine release induced by administration of $\Delta$ 9tetrahydrocannabinol (THC): selectivity to limbic striatum. Psychopharmacology 232, 2723-2729 (2015).

9 Pistis, M. et al. Delta(9)-tetrahydrocannabinol decreases extracellular GABA and increases extracellular glutamate and dopamine levels in the rat prefrontal cortex: an in vivo microdialysis study. Brain Res 948, 155-158 (2002). Mason, N. L. et al. Cannabis induced increase in striatal glutamate associated with loss of functional corticostriatal connectivity. European Neuropsychopharmacology 29, 247-256 (2018).

11 Colizzi, M. et al. Delta-9-tetrahydrocannabinol increases striatal glutamate levels in healthy individuals: implications for psychosis. Molecular psychiatry, doi:10.1038/s41380-019-0374-8 (2019).

12 Scofield, M. D. et al. The Nucleus Accumbens: Mechanisms of Addiction across Drug Classes Reflect the Importance of Glutamate Homeostasis. Pharmacological reviews 68, 816-871, doi:10.1124/pr.116.012484 (2016). Peter W. Kalivas, Ph.D. , and \& Nora D. Volkow, M.D. The Neural Basis of Addiction: A Pathology of Motivation and Choice. American Journal of Psychiatry 162, 1403-1413, doi:10.1176/appi.ajp.162.8.1403 (2005). van de Giessen, E. et al. Deficits in striatal dopamine release in cannabis dependence. Molecular psychiatry 22, 68 (2017). Bloomfield, M. A. P. et al. Dopaminergic Function in Cannabis Users and Its Relationship to Cannabis-Induced Psychotic Symptoms. Biological psychiatry 75, 470-478, doi:https://doi.org/10.1016/j.biopsych.2013.05.027 (2014). Leroy, C. et al. Striatal and extrastriatal dopamine transporter in cannabis and tobacco addiction: a high-resolution PET study. Addiction biology 17, 981-990 (2012).

Muetzel, R. L. et al. In vivo $1 \mathrm{H}$ magnetic resonance spectroscopy in young-adult daily marijuana users. Neurolmage: Clinical 2, 581-589 (2013)

Chang, L., Cloak, C., Yakupov, R. \& Ernst, T. Combined and independent effects of chronic marijuana use and HIV on brain metabolites. Journal of neuroimmune pharmacology : the official journal of the Society on Neurolmmune Pharmacology 1, 65-76, doi:10.1007/s11481-005-9005-z (2006). Prescot, A. P., Locatelli, A. E., Renshaw, P. F. \& Yurgelun-Todd, D. A. Neurochemical alterations in adolescent chronic marijuana smokers: a proton MRS study. Neurolmage 57, 69-75, doi:10.1016/j.neuroimage.2011.02.044 (2011).

Prescot, A. P., Renshaw, P. F. \& Yurgelun-Todd, D. A. gamma-Amino butyric acid and glutamate abnormalities in adolescent chronic marijuana smokers. Drug and alcohol dependence 129, 232-239, doi:10.1016/j.drugalcdep.2013.02.028 (2013). Hill, S. Y., Sharma, V. \& Jones, B. L. Lifetime use of cannabis from longitudinal assessments, cannabinoid receptor (CNR1) variation, and reduced volume of the right anterior cingulate. Psychiatry Research: Neuroimaging 255, 24-34 (2016). Filbey, F. M. et al. Long-term effects of marijuana use on the brain. Proceedings of the National Academy of Sciences 111 16913-16918, doi:10.1073/pnas.1415297111 (2014). Battistella, G. et al. Long-term effects of cannabis on brain structure. Neuropsychopharmacology 39, 2041-2048, doi:10.1038/npp.2014.67 (2014).

Hermann, D. et al. Dorsolateral Prefrontal Cortex N-Acetylaspartate/Total Creatine (NAA/tCr) Loss in Male Recreational Cannabis Users. Biological psychiatry 61, 1281-1289, doi:https://doi.org/10.1016/j.biopsych.2006.08.027 (2007). Cowan, R. L., Joers, J. M. \& Dietrich, M. S. N-acetylaspartate (NAA) correlates inversely with cannabis use in a frontal language processing region of neocortex in MDMA (Ecstasy) polydrug users: a $3 \mathrm{~T}$ magnetic resonance spectroscopy study. Pharmacology Biochemistry and Behavior 92, 105-110 (2009). Mashhoon, Y., Jensen, J. E., Sneider, J. T., Yurgelun-Todd, D. A. \& Silveri, M. M. Lower left thalamic myo-inositol levels associated with greater cognitive impulsivity in marijuana-dependent young men: preliminary spectroscopic evidence at 4T. Journal of addiction research \& therapy (2013).

Silveri, M. M., Jensen, J. E., Rosso, I. M., Sneider, J. T. \& Yurgelun-Todd, D. A. Preliminary evidence for white matter metabolite differences in marijuana-dependent young men using 2D J-resolved magnetic resonance spectroscopic imaging at 4 Tesla. Psychiatry research 191, 201-211, doi:10.1016/j.pscychresns.2010.10.005 (2011). 


\section{Reduced responsiveness of the reward system in chronic cannabis users}

Sung, Y. H. et al. Decreased frontal N-acetylaspartate levels in adolescents concurrently using both methamphetamine and marijuana. Behav Brain Res 246, 154-161 (2013).

Ramaekers, J. et al. Methylphenidate reduces functional connectivity of nucleus accumbens in brain reward circuit. Psychopharmacology (Berl) 229, 219-226, doi:10.1007/s00213-013-3105-x (2013).

Ramaekers, J. G. et al. Cannabis and cocaine decrease cognitive impulse control and functional corticostriatal connectivity in drug users with low activity DBH genotypes. Brain Imaging and Behavior 10, 1254-1263 (2016).

Ramaekers, J. G., Kauert, G., Theunissen, E., Toennes, S. W. \& Moeller, M. Neurocognitive performance during acute THC intoxication in heavy and occasional cannabis users. Journal of psychopharmacology 23, 266-277 (2009).

Hartley, S. et al. Effect of Smoked Cannabis on Vigilance and Accident Risk Using Simulated Driving in Occasional and Chronic Users and the Pharmacokinetic-Pharmacodynamic Relationship. Clinical chemistry, clinchem. 2018.299727 (2019).

Frahm, J. et al. Localized high-resolution proton NMR spectroscopy using stimulated echoes: initial applications to human brain in vivo. Magnetic resonance in medicine 9, 79-93 (1989).

Chao-Gan, Y. \& Yu-Feng, Z. DPARSF: A MATLAB Toolbox for "Pipeline" Data Analysis of Resting-State fMRI. Frontiers in systems neuroscience 4, 13, doi:10.3389/fnsys.2010.00013 (2010).

Dinges, D. F. \& Powell, J. W. Microcomputer analyses of performance on a portable, simple visual RT task during sustained operations. Behavior Research Methods, Instruments, \& Computers 17, 652-655, doi:10.3758/bf03200977 (1985).

Toennes, S. W., Ramaekers, J. G., Theunissen, E. L., Moeller, M. R. \& Kauert, G. F. Comparison of cannabinoid pharmacokinetic properties in occasional and heavy users smoking a marijuana or placebo joint. Journal of analytical toxicology 32, 470-477 (2008).

Cummings, J. L. Frontal-subcortical circuits and human behavior. Archives of neurology 50, 873-880 (1993).

Alexander, G. E., DeLong, M. R. \& Strick, P. L. Parallel organization of functionally segregated circuits linking basal ganglia and cortex. Annual review of neuroscience 9, 357-381 (1986).

Bonelli, R. M. \& Cummings, J. L. Frontal-subcortical circuitry and behavior. Dialogues in clinical neuroscience $9,141(2007)$. Perreault, M. L., Hasbi, A., O'Dowd, B. F. \& George, S. R. The dopamine D1-D2 receptor heteromer in striatal medium spiny neurons: evidence for a third distinct neuronal pathway in basal ganglia. Frontiers in neuroanatomy 5, 31 (2011).

Pierce, R. C. \& Kumaresan, V. The mesolimbic dopamine system: the final common pathway for the reinforcing effect of drugs of abuse? Neurosci Biobehav Rev 30, 215-238, doi:10.1016/j.neubiorev.2005.04.016 (2006).

Volkow, N. D., Wang, G. J., Fowler, J. S., Tomasi, D. \& Telang, F. Addiction: beyond dopamine reward circuitry. Proc Natl Acad Sci U S A 108, 15037-15042, doi:10.1073/pnas.1010654108 (2011).

Lupica, C. R., Riegel, A. C. \& Hoffman, A. F. Marijuana and cannabinoid regulation of brain reward circuits. British journal of pharmacology 143, 227-234, doi:10.1038/sj.bjp.0705931 (2004).

Breivogel, C. S. et al. Chronic delta9-tetrahydrocannabinol treatment produces a time-dependent loss of cannabinoid receptors and cannabinoid receptor-activated G proteins in rat brain. Journal of neurochemistry 73, 2447-2459 (1999).

Sim-Selley, L. J. Regulation of cannabinoid CB1 receptors in the central nervous system by chronic cannabinoids. Critical reviews in neurobiology 15, 91-119 (2003).

McKinney, D. L. et al. Dose-related differences in the regional pattern of cannabinoid receptor adaptation and in vivo tolerance development to delta9-tetrahydrocannabinol. The Journal of pharmacology and experimental therapeutics 324, 664-673, doi:10.1124/jpet.107.130328 (2008).

Sim, L. J., Hampson, R. E., Deadwyler, S. A. \& Childers, S. R. Effects of chronic treatment with delta9-tetrahydrocannabinol on cannabinoid-stimulated [35S]GTPgammaS autoradiography in rat brain. The Journal of neuroscience : the official journal of the Society for Neuroscience 16, 8057-8066 (1996).

Romero, J. et al. Atypical location of cannabinoid receptors in white matter areas during rat brain development. Synapse 26, 317-323 (1997).

Hirvonen, J. et al. Reversible and regionally selective downregulation of brain cannabinoid CB1 receptors in chronic daily cannabis smokers. Molecular psychiatry 17, 642-649, doi:10.1038/mp.2011.82 (2012).

Ceccarini, J. et al. [18F]MK-9470 PET measurement of cannabinoid CB1 receptor availability in chronic cannabis users. Addict Biol 20, 357-367, doi:10.1111/adb.12116 (2015).

D'Souza, D. C. et al. Rapid Changes in CB1 Receptor Availability in Cannabis Dependent Males after Abstinence from Cannabis. Biological psychiatry. Cognitive neuroscience and neuroimaging 1, 60-67, doi:10.1016/j.bpsc.2015.09.008 (2016).

Gonzalez, S., Cebeira, M. \& Fernandez-Ruiz, J. Cannabinoid tolerance and dependence: a review of studies in laboratory animals. Pharmacology, biochemistry, and behavior 81, 300-318, doi:10.1016/j.pbb.2005.01.028 (2005).

Pope, H. G., Jr, Gruber, A. J., Hudson, J. I., Huestis, M. A. \& Yurgelun-Todd, D. Neuropsychological Performance in Longterm Cannabis Users. JAMA Psychiatry 58, 909-915, doi:10.1001/archpsyc.58.10.909 (2001).

Sneider, J. T., Mashhoon, Y. \& Silveri, M. M. A Review of Magnetic Resonance Spectroscopy Studies in Marijuana using Adolescents and Adults. Journal of addiction research \& therapy Suppl 4. 


\section{Chapter 3}

55 Crean, R. D., Crane, N. A. \& Mason, B. J. An evidence based review of acute and long-term effects of cannabis use on executive cognitive functions. Journal of addiction medicine 5, 1-8, doi:10.1097/ADM.0b013e31820c23fa (2011).

56 Harte, M. K., Bachus, S. B. \& Reynolds, G. P. Increased N-acetylaspartate in rat striatum following long-term administration of haloperidol. Schizophrenia research 75, 303-308, doi:10.1016/j.schres.2004.11.001 (2005).

57 Malhi, G. S., Valenzuela, M., Wen, W. \& Sachdev, P. Magnetic resonance spectroscopy and its applications in psychiatry. The Australian and New Zealand journal of psychiatry 36, 31-43, doi:10.1046/j.1440-1614.2002.00992.x (2002).

58 Plitman, E. et al. Elevated Myo-Inositol, Choline, and Glutamate Levels in the Associative Striatum of Antipsychotic-Naive Patients With First-Episode Psychosis: A Proton Magnetic Resonance Spectroscopy Study With Implications for Glial Dysfunction. Schizophrenia bulletin 42, 415-424, doi:10.1093/schbul/sbv118 (2016).

59 Jessen, F. et al. N-acetylaspartylglutamate (NAAG) and N-acetylaspartate (NAA) in patients with schizophrenia. Schizophrenia bulletin 39, 197-205, doi:10.1093/schbul/sbr127 (2013). 


\section{Supplemental Information}

\section{Material and Methods}

Participants. Participants were recruited through advertisements around Maastricht University. Inclusion criteria were: age, 18-40 years; occasional cannabis use for the occasional group, ranging between 1 time a month and 3 times a week for the past year OR chronic cannabis use for the chronic group, using at least 4 times a week for the past year; normal weight, body mass index between 18 and $28 \mathrm{~kg} / \mathrm{m} 2$; free from psychotropic medication; good physical health, including absence of major medical, endocrine, and neurological conditions; and written informed consent. Exclusion criteria were: history of drug abuse (other than the use of cannabis) or addiction, which were determined by medical questionnaires and examination; pregnancy or lactation; health issues including hypertension (diastolic >90 and systolic $>140$ ), cardiac dysfunction, and liver dysfunction; current or history of psychiatric disorders; previous experience of serious side effects to cannabis; and MRI contraindications. Before inclusion, subjects were screened and examined by a study physician, who checked for general health, conducted a resting ECG, and took blood and urine samples in which hematology, clinical chemistry, urine, and virology analyses were conducted. Participant demographic data can be found in Table S1.

A permit for obtaining, storing, and administering cannabis was obtained from the Dutch Drug Enforcement Administration. Participants were financially compensated for their participation in the study.

Administration. Treatment orders and doses were randomly assigned to subjects according to a balanced, block design. The dosage of cannabis was tailored to individual participants in order to reach $300 \mu \mathrm{g} / \mathrm{kg}$ bodyweight $\mathrm{THC}$, which has previously been found to be well tolerated by subjects with an average experience of cannabis use ${ }^{1,2}$.

Both treatments were administered through a Volcano vaporizer (Storz \& Bickel Volcano $\left.{ }^{\circledR}\right)$, with participants inhaling equal amounts of each while lying in the MRI scanner. The treatments were vaporized at $225^{\circ} \mathrm{C}$ and the vapor was stored in a polythene bag equipped with a mouth piece. Participants were instructed to place the mouth piece to their lips and inhale deeply, holding their breath for 10 seconds, and then exhaling. Participants repeated this procedure until balloon was empty. Participants were instructed to inhale the entire volume of the balloon within 5 minutes, according to a standardized procedure.

Procedures. Participants were familiarized with the test day procedures on a separate training day prior to the treatment conditions. Participants in the OU group were instructed to refrain from drug use, including cannabis, ( $\geq 7$ days) and alcohol use ( $\geq 24$ hours) prior to their testing day; whereas participants in the chronic group were given the same instructions, however were 
allowed to use cannabis up until 24 hours prior to their testing day. On arrival on a test day, absence of drug and alcohol were assessed via a urine drug screen and a breath alcohol screen. An additional pregnancy test was given if participants were female. If all tests were found to be negative (except for cannabis in the chronic group), participants were allowed to proceed, and a venal catheter was placed.

Psychomotor vigilance task. This study used a shortened, 5-minute version of the task on three consecutive time points after treatment administration. During the task, the participant is instructed to press a button as soon as the stimulus appears (red circle). The outcome variables of the PVT are: mean RT, the mean RT for all trials; optimum response domain, the fastest $10 \%$ of response times for all trials (i.e. average of the fastest 10\% RT); number of lapses, the number of response times greater than or equal to $500 \mathrm{~ms}$ for all trials (i.e. lapse); and lapse domain, the slowest $10 \%$ of reciprocal response times for all trials (i.e. average of the slowest $10 \% 1 /$ RT) ${ }^{3}$. For this study, only number of lapses was considered.

Pharmacokinetic measures. Blood samples were centrifuged and serum was frozen at $-20{ }^{\circ} \mathrm{C}$ until analyses for pharmacokinetic assessments. Cannabinoid concentrations were determined using a validated and proficiency test approved forensic routine method consisting of an automated solidphase extraction and gas chromotrography with tandem mass spectrometric detection with a limit of quantification of $0.3 \mathrm{ng} / \mathrm{ml}$ or less ${ }^{4}$.

MRS Data Acquisition and Quality. Single-voxel proton magnetic resonance spectroscopy (MRS) measurements were performed on a MAGNETOM 7T MR scanner (Siemens Healthineers, Erlangen, Germany) with a whole-body gradient set (SC72; maximum amplitude, $70 \mathrm{mT} / \mathrm{m}$; maximum slew rate, $200 \mathrm{~T} / \mathrm{m} / \mathrm{s}$ ) and using an single-channel transmit/32-channel receive head coil (Nova Medical, Wilmington, MA, USA). Spectroscopic voxels of interest were placed by a trained operator at the Anterior Cingulate Cortex (ACC) (voxel size $=25 \times 20 \times 17 \mathrm{~mm}^{3}$ ) and the right striatum (voxel size $=20 \times 20 \times 20 \mathrm{~mm}^{3}$ ). Spectra were acquired with stimulated echo acquisition mode $(\mathrm{STEAM}){ }^{5}$ sequence using the following parameters: $\mathrm{TE}=6.0 \mathrm{~ms}, \mathrm{TM}=10.0 \mathrm{~ms}, \mathrm{TR}=5.0 \mathrm{~s}$, $\mathrm{NA}=64$, flip angle $=90^{\circ}, \mathrm{RF}$ bandwidth $=4.69 \mathrm{kHz}$, RF centred at $2.4 \mathrm{ppm}$, receive bandwidth = $4.0 \mathrm{kHz}$, vector size $=2048,16$-step phase cycling, acquisition time $=5: 20 \mathrm{~min}$. Water suppression was achieved by variable power RF pulses with optimised relaxation delays (VAPOR) ${ }^{6}$. In addition, a complete phase cycle of measurements was acquired without the water suppression RF pulses to record a water peak reference for eddy current correction ${ }^{7}$ and absolute metabolite concentration calibration ${ }^{8,9}$. Before the spectroscopy measurements, a 3D-GRE dual-echo fieldmap $\left(\mathrm{TE}_{1}=1.00 \mathrm{~ms}, \mathrm{TE}_{2}=2.98 \mathrm{~ms}, \mathrm{TR}=20.0 \mathrm{~ms}\right.$, flip angle $=8^{\circ}$, voxel size $=3 \mathrm{~mm}$ isotropic, matrix size $=84 \times 84 \times 56$, bandwidth $=1450 \mathrm{~Hz} /$ pixel, acquisition time $=2: 24 \mathrm{~min}$ ) was acquired and used 
calculate the shim currents required to homogenise the static magnetic field in the spectroscopic voxels of interest.

The spectra were analysed with LCModel version 6.3-1H using a GAMMA ${ }^{10}$ simulated basis set which includes Alanine (Ala), Ascorbic Acid (Asc), Aspartate (Asp), Creatine ( $\mathrm{Cr}$ ), $\mathrm{Y}$-Aminobutyric Acid (GABA), Glucose (Glc), Glutamate (Glu), Glutamine (Gln), Glycerophosphocholine (GPC), Glutathione (GSH), Glycine (Glyc), Lactate (Lac), Myo-Inositol (ml), N-Acetyl Aspartate (NAA), NAcetyl Aspartyl Glutamate (NAAG), Phosphocreatine (PCr), Phosphorylcholine (PCh), Phosphorylethanolamine (PE), Scyllo-Inositol (Scyllo), and Taurine (Tau) ${ }^{11}$. The metabolite basis set also includes an in vivo Macromolecules (MMol) spectrum which was collected using a metabolites suppressed double inversion recovery (DIR) STEAM with the same parameters as above and $\mathrm{Tl}_{1}=2.09 \mathrm{~s}$ and $\mathrm{TI}_{2}=0.52 \mathrm{~s}^{12}$.

Anatomical ( $T_{1}$-weighted) images were acquired using magnetisation-prepared 2 rapid acquisition gradient-echo (MP2RAGE) ${ }^{13}$ sequence $\left(T R=4.5 \mathrm{~s}, \mathrm{TE}=2.39 \mathrm{~ms}, \mathrm{Tl}_{1}=0.90 \mathrm{~s}, \mathrm{Tl}_{2}=2.75\right.$ $\mathrm{s}$, flip angle ${ }_{1}=5^{\circ}$, flip angle $e_{2}=3^{\circ}$, voxel size $=0.9 \mathrm{~mm}$ isotropic, matrix size $=256 \times 256 \times 192$, phase partial Fourier $=6 / 8$, GRAPPA factor $=3$ with 24 reference lines, bandwidth $=250 \mathrm{~Hz} /$ pixel, acquisition time $=6: 00 \mathrm{~min})$. Tissue probability maps for grey matter (GM), white matter (WM) and cerebrospinal fluid (CSF) were generated from the $T_{1}$-weighted anatomical images using FSLFAST ${ }^{14}$. To ensure data quality and reliable metabolite estimation, only absolute metabolite values with a Cramer-Rao lower bound below 20\% and a signal-to-noise ratio greater than 10 were considered (Provencher, 2001). An example spectrum can be found in Figure S2, and mean SNR and \%CRLB values in Table S6.

fMRI data preprocessing and functional connectivity. Resting state image preprocessing were conducted using SPM8 (Statistical Parametric Mapping, Wellcome Trust Centre for Neuroimaging, Institute of Neurology, University College London) Preprocessing steps included: motion correction (registered to the first image with second degree B-spline interpolation), coregistration (linking of functional to anatomical scans), and spatial normalization (the mean EPI image of each session was matched to SPM8's EPI template in Montreal Neurological Institute [MNI] space) where after the parameters were applied to all images of that session. During normalization voxel size was $1.5 \times 1.5 \times 1.5 \mathrm{~mm}$. Finally, the data were smoothed at 3mm Gaussian kernel.

Linear trends of time courses were removed followed by low band-pass filtered (0.01-0.08 $\mathrm{HZ}$ ) of the preprocessed data to remove 'noise' attributable to physiological parameters. Nuisance covariates (motion parameters, white matter signal, CSF signal) were also removed. In order to indirectly assess dopamine neurotransmission, two spheres (4 $\mathrm{mm}$ radius) were created that were located (in MNI space) in the left $(-9,9,-9)$ and right $(9,9,-9)$ NAc. Average time courses were obtained for each sphere separately and correlational analysis was performed voxel wise to 
generate functional connectivity maps for each sphere. The correlation coefficient map was converted into $z$ maps by Fisher's r-to-z transform to improve normality ${ }^{15,16}$. This is in accordance with previous studies investigating drug induced changes in functional connectivity ${ }^{17,18}$.

Furthermore, as we were interested in FC within the reward circuit, ROI-to-ROI FC was computed according to the same aforementioned procedure, between areas including: NAC (-9, 9, -9 , radius $4 \mathrm{~mm})$, MDN $(-9,-19,-6,4 \mathrm{~mm}), \mathrm{VP}(-20,-4,-2,4 \mathrm{~mm})$ and the $\mathrm{MC}(-4,-18,44,4 \mathrm{~mm})$. NAc, MDN, and VP seed locations were in agreement with structural and functional subdivisions of these brain regions that were validated in previous work ${ }^{19-21}$. The MC was based off of the first FC analysis. Analysis was performed between the NAc and MDN, NAc and VPN, MDN and VPN, $M D N$ and $M C$, and $M C$ and NAc. Treatment change was averaged across the 2 time points [Average(THC time point 1- placebo time point 1); (THC time point 2 - placebo time point 2)].

Statistical analysis of FC data. Functional connectivity data (i.e. correlation coefficient maps for each individual in each treatment condition at each time point) were analyzed in a GLM model in SPM 12. In the first GLM, data entered a full factorial model with treatment (THC and placebo) and time point (2 levels) as within-subject factors and group (occasional and chronic user group) as a between-subject factor. From this model main effects of treatment were identified for the occasional user group, but not the chronic user group. Maps were corrected for multiple comparisons at the cluster level using the family wise error correction (FWE).

Correlational analyses. Correlation analyses were conducted to further investigate the relationship between THC induced changes in brain and behavior. In order to reduce the number of comparisons, only variables significantly affected by THC were assessed (table 57). For the psychomotor vigilance task, only number of lapses was used as a variable of sustained attention. For the voxel wise correlation analysis (Table S8) between NAc FC and behavioral outcomes, individual treatment maps (placebo > THC) were entered into one-sample $t$-tests in SPM, with the average change scores of subjective high, and number of attention lapses. Maps were corrected for multiple comparisons at the cluster level using the family wise error correction (FWE). Average mean voxel activation of SPM identified clusters were put into SPSS and Pearson's correlations were performed to get correlation strengths. 
Reduced responsiveness of the reward system in chronic cannabis users

Table S1. Mean subject characteristics (SD) and history of drug use for occasional and chronic cannabis users that completed the study $(\mathrm{N}=24)$.

\begin{tabular}{|c|c|c|c|c|c|}
\hline Variable & $\begin{array}{l}\text { Occasional } \\
\text { Users }\end{array}$ & Chronic Users & Value & $d f$ & $P$ value \\
\hline Gender (male/female), n, total & $5 / 7,12$ & $9 / 3,12$ & $x 2=2.74^{\ddagger}$ & 1 & 0.10 \\
\hline Age, years & $22.5(2.54)$ & $21.83(2.25)$ & $t=.681^{\dagger}$ & 22 & 0.50 \\
\hline History of cannabis use, years & $5.50(2.71)$ & $5.33(1.78)$ & $t=.178^{\dagger}$ & 22 & 0.86 \\
\hline $\begin{array}{l}\text { Frequency of cannabis use, per } \\
\text { week }\end{array}$ & $1.21(0.80)$ & $6.63(1.40)$ & $t=-11.62^{\dagger}$ & 22 & $0.00 *$ \\
\hline $\begin{array}{l}\text { Alcohol consumption, glasses per } \\
\text { week }\end{array}$ & $7.29(7.05)$ & $3.17(2.32)$ & $t=1.93^{\dagger}$ & 22 & 0.07 \\
\hline Caffeine consumption (per week) & $8.25(7.71)$ & $8.88(6.05)$ & $t=-0.22^{+}$ & 22 & 0.83 \\
\hline Nicotine consumption, per week & $16.33(22.07)$ & $19.29(28.23)$ & $t=-1.25^{+}$ & 22 & 0.22 \\
\hline Occasional use of other drugs, $n$ & 9 & 9 & $x^{2}=0.00^{\ddagger}$ & 1 & 1.00 \\
\hline
\end{tabular}

*Significant $P$ values; ${ }^{\dagger}$ Independent $t$ test; ${ }^{\ddagger} \chi 2$ test for frequency data 


\section{Chapter 3}

Table S2. Time course for mean (S.E.) concentrations of THC and its metabolites in serum (ng/ml) following smoking THC, as assessed by gas chromatography-mass spectrometry (GC-MS).

\begin{tabular}{|c|c|c|c|c|c|c|}
\hline \multirow{3}{*}{$\begin{array}{l}\text { Time Relative } \\
\text { to Smoking }\end{array}$} & \multicolumn{6}{|c|}{ Serum (GC-MS) } \\
\hline & \multicolumn{3}{|c|}{ Occasional Users } & \multicolumn{3}{|c|}{ Chronic Users } \\
\hline & THC & 11-OH-THC & $\mathrm{THC}-\mathrm{COOH}$ & THC & 11-OH-THC & $\mathrm{THC}-\mathrm{COOH}$ \\
\hline 0 & $0.00(.00)^{*}$ & $0.02(.02)$ & $1.48(0.71)$ & $3.48(.89)$ & $1.55(.38)$ & 47.44 (14.33) \\
\hline 6 & 7.80 (1.69) & $1.70(0.43)$ & $9.50(2.23)$ & $15.86(3.48)$ & $3.84(1.13)$ & 48.81 (14.19) \\
\hline 28 & $2.86(0.55)$ & $1.03(0.22)$ & $8.37(1.82)$ & $6.66(1.55)$ & $2.10(.62)$ & 45.53 (15.43) \\
\hline 50 & $2.07(0.34)$ & $0.86(0.20)$ & 7.55 (1.55) & $7.10(1.44)$ & $2.07(.46)$ & $45.72(12.23)$ \\
\hline 67 & $2.22(0.52)$ & $1.00(0.23)$ & 8.60 (1.94) & 5.67 (1.48) & $1.67(.47)$ & 36.90 (13.27) \\
\hline
\end{tabular}

*one participant exhibited a THC concentration of $1.1 \mathrm{ng} / \mathrm{ml}$, which was not regarded as indicative of recent use due to a low THC-OH concentration $(0.2 \mathrm{ng} / \mathrm{ml})$. 
OU $\quad \mathrm{CU}$

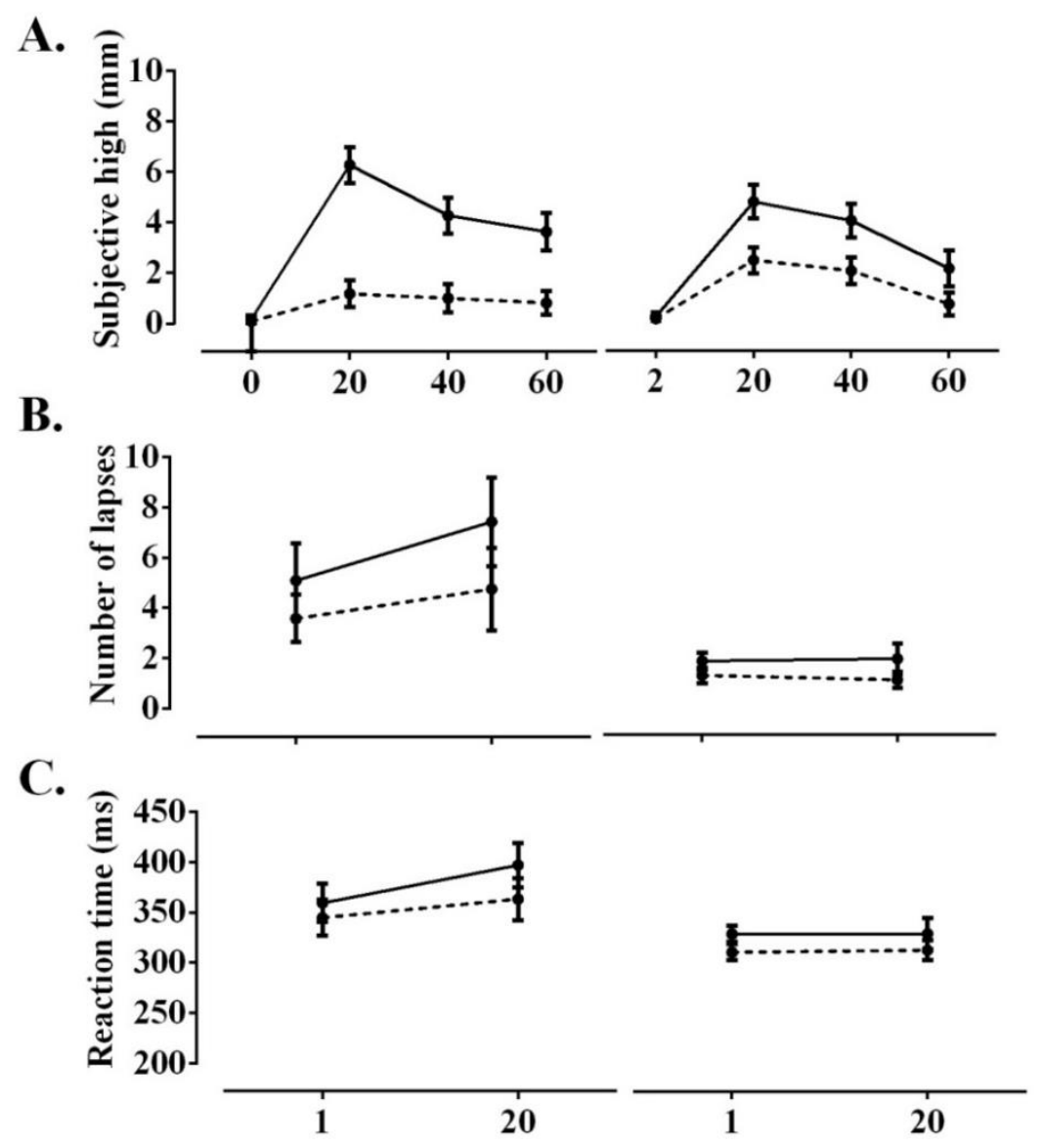

Time Relative to Smoking (minutes)

-• Placebo •- THC

Figure S1. Subjective and cognitive effects. A. Occasional and chronic users mean (SE) subjective high for both treatments (THC vs placebo) as a function of time relative to smoking $(0,20,40$, and 60 minutes). B. Occasional and chronic users mean (SE) reaction time, and C. number of lapses (RT>500 ms) for both treatments (THC vs placebo) as a function of time relative to smoking (1,20, and 40 minutes). $\mathrm{OU}=$ occasional user; $\mathrm{CU}=$ chronic user 
Chapter 3

Table S3. Mean (SE) metabolite concentrations for occasional and chronic users.

\begin{tabular}{|c|c|c|c|c|c|c|c|c|c|c|c|}
\hline & & \multicolumn{2}{|c|}{$\mathrm{Glu} / \mathrm{tCr}$} & \multicolumn{2}{|c|}{$\mathrm{GABA} / \mathrm{tCr}$} & \multicolumn{2}{|c|}{$\mathrm{NAA}+\mathrm{NAAG} / \mathrm{tCr}$} & \multicolumn{2}{|c|}{$\mathrm{ml} / \mathrm{tCr}$} & \multicolumn{2}{|c|}{$\mathrm{GPC}+\mathrm{PCh} / \mathrm{tCr}$} \\
\hline & \multicolumn{11}{|c|}{ Occasional Users } \\
\hline & Time & THC & Placebo & THC & Placebo & THC & Placebo & THC & Placebo & THC & Placebo \\
\hline \multirow{2}{*}{ 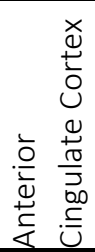 } & 1 & $\begin{array}{l}1.04 \\
(.05)\end{array}$ & $\begin{array}{l}1.05 \\
(.03)\end{array}$ & $\begin{array}{l}.18 \\
(.01)\end{array}$ & $\begin{array}{l}.17 \\
(.04)\end{array}$ & $\begin{array}{l}1.15 \\
(.06)\end{array}$ & $\begin{array}{l}1.20 \\
(.05)\end{array}$ & $\begin{array}{l}.87 \\
(.02)\end{array}$ & $\begin{array}{l}.82 \\
(.03)\end{array}$ & $\begin{array}{l}0.18 \\
(.01)\end{array}$ & $\begin{array}{l}.19 \\
(.01)\end{array}$ \\
\hline & 2 & $\begin{array}{l}1.04 \\
(.04)\end{array}$ & $\begin{array}{l}1.05 \\
(.03)\end{array}$ & $\begin{array}{l}.15 \\
(.01)\end{array}$ & $\begin{array}{l}.14 \\
(.01)\end{array}$ & $\begin{array}{l}1.17 \\
(.05)\end{array}$ & $\begin{array}{l}1.17 \\
(.04)\end{array}$ & $\begin{array}{l}.86 \\
(.02)\end{array}$ & $\begin{array}{l}.81 \\
(.03)\end{array}$ & $\begin{array}{l}0.17 \\
(.01)\end{array}$ & $\begin{array}{l}.19 \\
(.01)\end{array}$ \\
\hline \multirow[b]{2}{*}{ 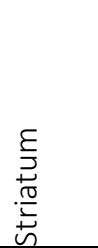 } & 1 & $\begin{array}{l}1.03 \\
(.04)\end{array}$ & $\begin{array}{l}.99 \\
(.05)\end{array}$ & $\begin{array}{l}.20 \\
(.01)\end{array}$ & $\begin{array}{l}.19 \\
(.02)\end{array}$ & $\begin{array}{l}1.45 \\
(.05)\end{array}$ & $\begin{array}{l}1.36 \\
(.06)\end{array}$ & $\begin{array}{l}.58 \\
(.03)\end{array}$ & $\begin{array}{l}.49 \\
(.03)\end{array}$ & $\begin{array}{l}.15 \\
(.01)\end{array}$ & $\begin{array}{l}.16 \\
(.01)\end{array}$ \\
\hline & 2 & $\begin{array}{l}1.14 \\
(.05)\end{array}$ & $\begin{array}{l}.93 \\
(.02)\end{array}$ & $\begin{array}{l}.30 \\
(.04)\end{array}$ & $\begin{array}{l}.19 \\
(.03)\end{array}$ & $\begin{array}{l}1.58 \\
(.06)\end{array}$ & $\begin{array}{l}1.34 \\
(.05)\end{array}$ & $\begin{array}{l}.61 \\
(.04)\end{array}$ & $\begin{array}{l}.52 \\
(.03)\end{array}$ & $\begin{array}{l}.16 \\
(.01)\end{array}$ & $\begin{array}{l}.15 \\
(.01)\end{array}$ \\
\hline \multicolumn{12}{|c|}{ Chronic Users } \\
\hline \multirow{2}{*}{ 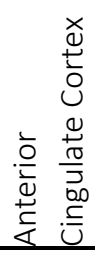 } & 1 & $\begin{array}{l}1.06 \\
(.02)\end{array}$ & $\begin{array}{l}1.05 \\
(.06)\end{array}$ & $\begin{array}{l}.15 \\
(.01)\end{array}$ & $\begin{array}{l}.17 \\
(.03)\end{array}$ & $\begin{array}{l}1.18 \\
(.03)\end{array}$ & $\begin{array}{l}1.19 \\
(.06)\end{array}$ & $\begin{array}{l}.76 \\
(.05)\end{array}$ & $\begin{array}{l}.90 \\
(.05)\end{array}$ & $\begin{array}{l}.18 \\
(.01)\end{array}$ & $\begin{array}{l}.19 \\
(.01)\end{array}$ \\
\hline & 2 & $\begin{array}{l}1.08 \\
(.03)\end{array}$ & $\begin{array}{l}1.05 \\
(.05)\end{array}$ & $\begin{array}{l}.15 \\
(.01)\end{array}$ & $\begin{array}{l}.15 \\
(.01)\end{array}$ & $\begin{array}{l}1.25 \\
(.03)\end{array}$ & $\begin{array}{l}1.24 \\
(.04)\end{array}$ & $\begin{array}{l}.79 \\
(.04)\end{array}$ & $\begin{array}{l}.89 \\
(.85)\end{array}$ & $\begin{array}{l}.18 \\
(.01)\end{array}$ & $\begin{array}{l}.18 \\
(.01)\end{array}$ \\
\hline \multirow[b]{2}{*}{ 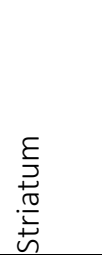 } & 1 & $\begin{array}{l}1.02 \\
(.04)\end{array}$ & $\begin{array}{l}1.08 \\
(.07)\end{array}$ & $\begin{array}{l}.24 \\
(.03)\end{array}$ & $\begin{array}{l}.26 \\
(.02)\end{array}$ & $\begin{array}{l}1.36 \\
(.08)\end{array}$ & $\begin{array}{l}1.34 \\
(.05)\end{array}$ & $\begin{array}{l}.56 \\
(.05)\end{array}$ & $\begin{array}{l}.57 \\
(.05)\end{array}$ & $\begin{array}{l}.14 \\
(.01)\end{array}$ & $\begin{array}{l}.16 \\
(.01)\end{array}$ \\
\hline & 2 & $\begin{array}{l}.97 \\
(.03)\end{array}$ & $\begin{array}{l}1.07 \\
(.06)\end{array}$ & $\begin{array}{l}.20 \\
(.02)\end{array}$ & $\begin{array}{l}.24 \\
(.04)\end{array}$ & $\begin{array}{l}1.44 \\
(.05)\end{array}$ & $\begin{array}{l}1.35 \\
(.05)\end{array}$ & $\begin{array}{l}.50 \\
(.03)\end{array}$ & $\begin{array}{l}.53 \\
(.03)\end{array}$ & $\begin{array}{l}.16 \\
(.01)\end{array}$ & $\begin{array}{l}.15 \\
(.01)\end{array}$ \\
\hline
\end{tabular}


Table S4. Significant decrements in functional connectivity of left nucleus accumbens, relative to placebo in the occasional group.

\begin{tabular}{|c|c|c|c|c|c|c|c|}
\hline & $R$ or $L$ & BA & k & $x$ & y & z & $\begin{array}{l}\text { P value } t_{3.20} \\
\text { FWE } \\
\text { cluster }\end{array}$ \\
\hline Cluster & & & & & & & corrected \\
\hline Precuneus & $L$ & 5 & 71 & -6 & -50 & 56 & .000 \\
\hline Middle Frontal Gyrus & $\mathrm{R}$ & 46 & 22 & 34 & 38 & 30 & .016 \\
\hline Middle Frontal Gyrus & $L$ & 6 & 19 & -38 & 6 & 62 & .043 \\
\hline Superior Frontal Gyrus & $L$ & 6 & 48 & -24 & 6 & 64 & .000 \\
\hline Precentral Gyrus & $\mathrm{L}$ & 6 & 39 & -28 & -26 & 68 & .000 \\
\hline Precentral Gyrus & $L$ & 6 & 39 & -48 & -4 & 42 & .000 \\
\hline Precentral Gyrus & $\mathrm{R}$ & 6 & 24 & 62 & 8 & 18 & .000 \\
\hline Precentral Gyrus & $\mathrm{R}$ & 6 & 24 & 54 & -2 & 46 & .008 \\
\hline Supplementary Motor Area & $\mathrm{L}$ & 6 & 24 & -6 & 8 & 48 & .008 \\
\hline Supplementary Motor Area & $\mathrm{R}$ & 6 & 24 & 6 & 12 & 66 & .008 \\
\hline Supplementary Motor Area & $\mathrm{R}$ & 6 & 44 & 8 & -6 & 68 & .000 \\
\hline Rolandic Operculum & $\mathrm{R}$ & 6 & 44 & 62 & 8 & 10 & .000 \\
\hline \multirow[t]{5}{*}{ Rolandic Operculum } & $L$ & 48 & 108 & -58 & 4 & 4 & .000 \\
\hline & $\mathrm{L}$ & 48 & 115 & -36 & 18 & 20 & .000 \\
\hline & $\mathrm{L}$ & 48 & 20 & -34 & 8 & 0 & .000 \\
\hline & $L$ & 48 & 21 & -30 & -30 & 12 & .022 \\
\hline & $\mathrm{R}$ & 48 & 52 & 44 & 12 & 18 & .000 \\
\hline Midcingulate Area & $\mathrm{R}$ & 23 & 96 & 6 & -24 & 46 & .000 \\
\hline Midcingulate Area & $L$ & 23 & 38 & -4 & -18 & 44 & .000 \\
\hline Postcentral Gyrus & $R$ & 43 & 57 & 60 & 2 & 24 & .000 \\
\hline Postcentral Gyrus & $L$ & 3 & 26 & -48 & -20 & 58 & .000 \\
\hline Inferior Parietal Lobule & $L$ & 2 & 28 & -48 & -40 & 58 & .002 \\
\hline Inferior Parietal Lobule & $L$ & 40 & 140 & -58 & -46 & 42 & .000 \\
\hline Supramarginal Gyrus & $L$ & 40 & 71 & -60 & -36 & 32 & .000 \\
\hline Calcarine Sulcus & $\mathrm{R}$ & 17 & 36 & 18 & -62 & 6 & .000 \\
\hline
\end{tabular}




\section{Chapter 3}

Middle Occipital Gyrus

$\begin{array}{llllll}\mathrm{L} & 19 & 19 & -38 & -86 & 24 \\ \mathrm{~L} & 19 & 36 & -40 & -88 & -14\end{array}$

.043

$-14$

.000

Statistical threshold: $\mathrm{P}<0.001$ (uncorrected). MNI coordinates of peak voxels for each cluster are given. $\mathrm{BA}=$ brodmann area 
Reduced responsiveness of the reward system in chronic cannabis users

Table S5. Testing day schedule

\begin{tabular}{|c|c|}
\hline Time after treatment (minutes) & Procedure \\
\hline \multicolumn{2}{|l|}{ Baseline } \\
\hline \multirow[t]{2}{*}{0} & $\begin{array}{l}\text { Urine sample (drug screen; pregnancy test); Alcohol breath } \\
\text { test }\end{array}$ \\
\hline & Vital signs; Questionnaires; Blood sample (S1) \\
\hline \multicolumn{2}{|l|}{ In Scanner } \\
\hline \multirow[t]{2}{*}{0} & Anatomical scan \\
\hline & Administration $(300 \mu \mathrm{g} / \mathrm{kg})$ \\
\hline 1 & MRS (ACC); PVT \\
\hline 6 & Blood sample (S2) \\
\hline 8 & MRS (striatum) \\
\hline 15 & Resting state scan \\
\hline 20 & Questionnaires \\
\hline 22 & MRS (ACC); PVT \\
\hline 28 & Blood sample (S3) \\
\hline 30 & MRS (striatum) \\
\hline 36 & Resting state scan \\
\hline 42 & Questionnaires \\
\hline 50 & Blood sample (S4) \\
\hline \multicolumn{2}{|l|}{ Outside of scanner } \\
\hline 68 & Questionnaires \\
\hline 72 & Blood sample (S5) \\
\hline
\end{tabular}




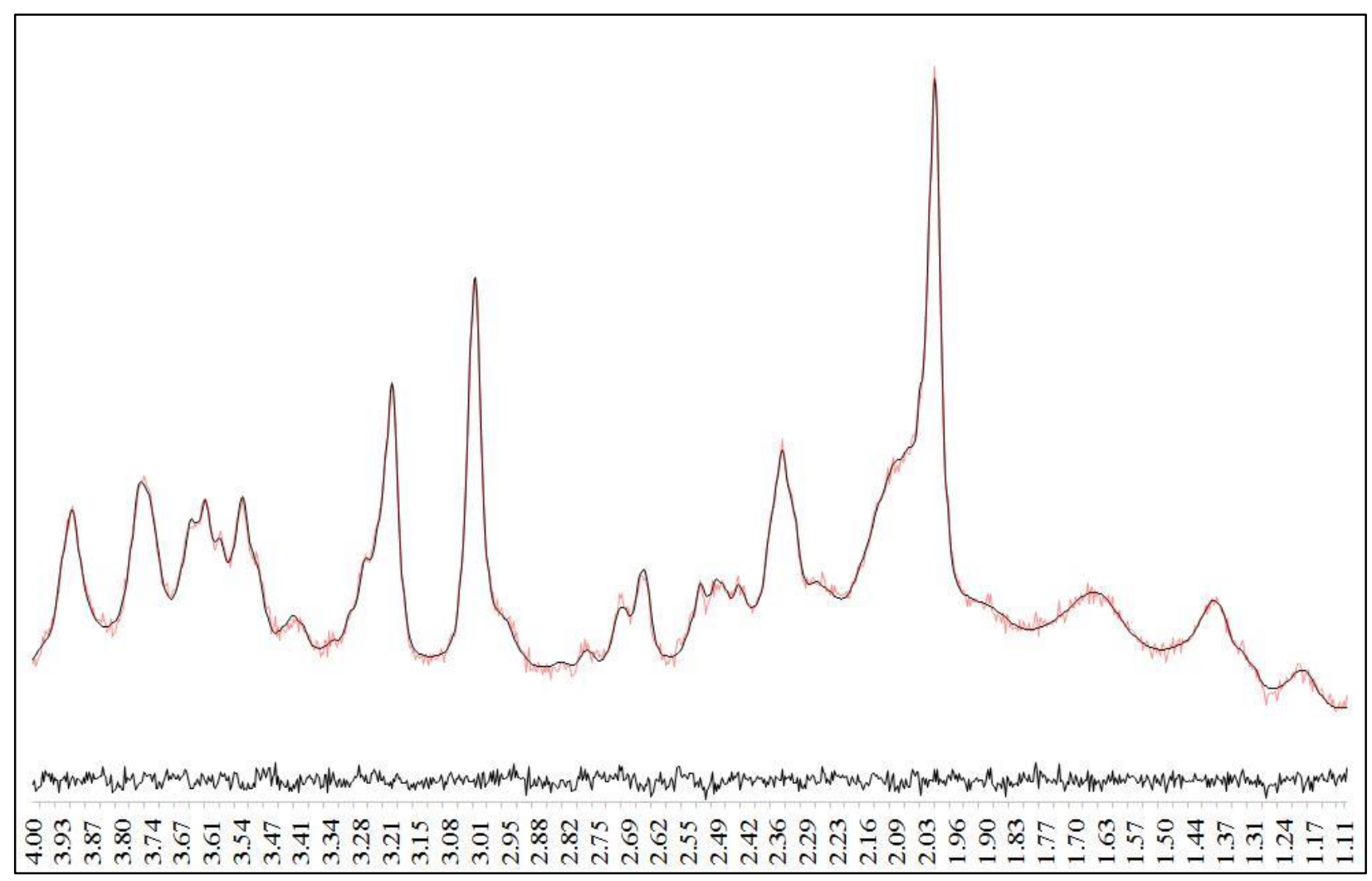

Figure S2. Example LC-Model fitted 1H-MRS data recorded from one participant. The black line spectra corresponds to the phased 1H-MRS data with the LC-Model fits overlaid (red). The residual spectra (raw data minus the LC-Model fit) are displayed below the spectrum. 
Reduced responsiveness of the reward system in chronic cannabis users

Table S6. Mean (min-max) ratios for signal to noise (SNR) and \%CRLB per group, per condition, and per timepoint. Only data points with an SNR $>10$ and a CRLB $<20 \%$ were included in the final analysis.

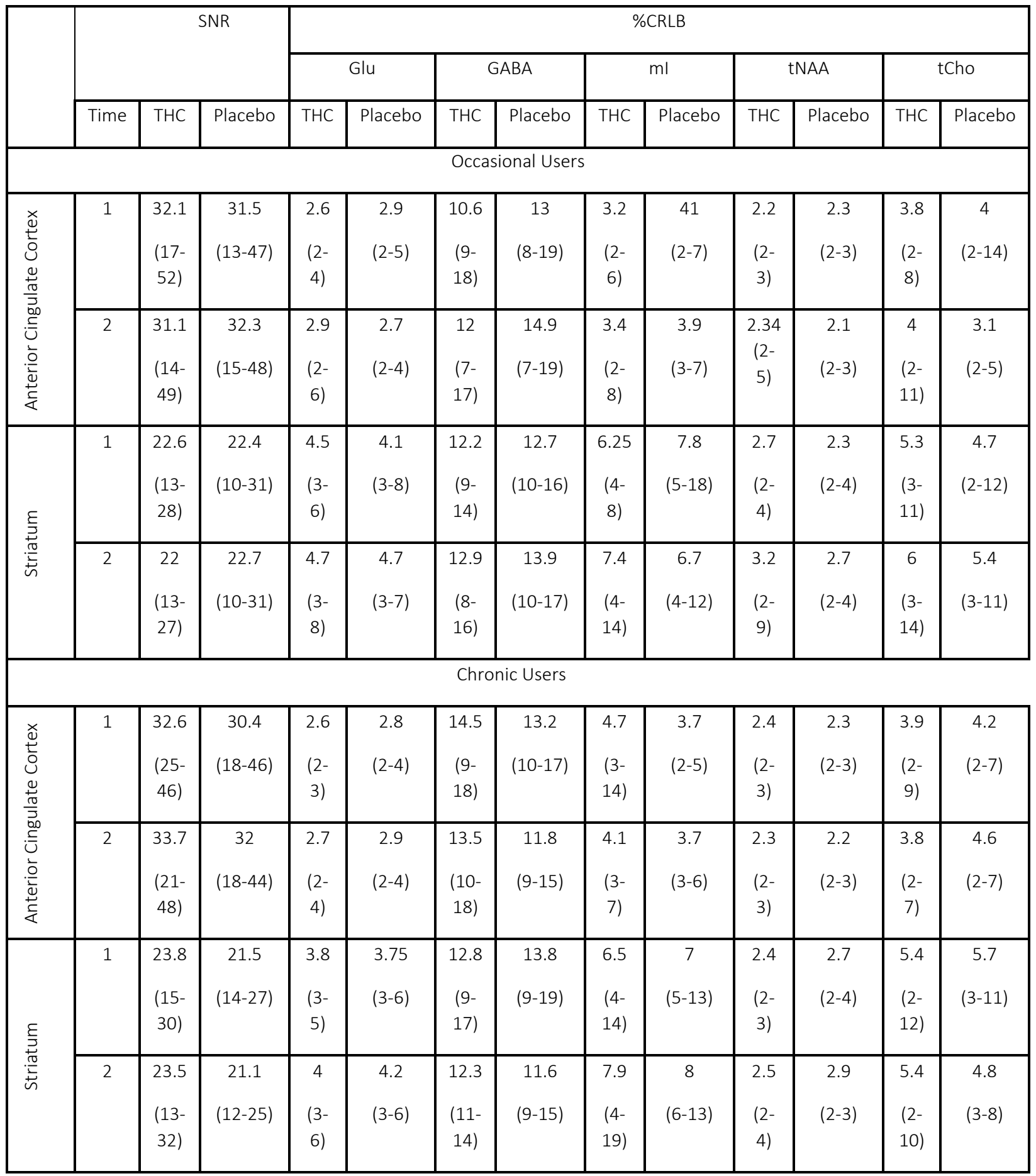




\section{Chapter 3}

Table S7. Correlations conducted between absolute average change scores (THC-placebo) of variables that were shown to be significantly affected by THC. OU = occasional user; $\mathrm{CU}=$ chronic user

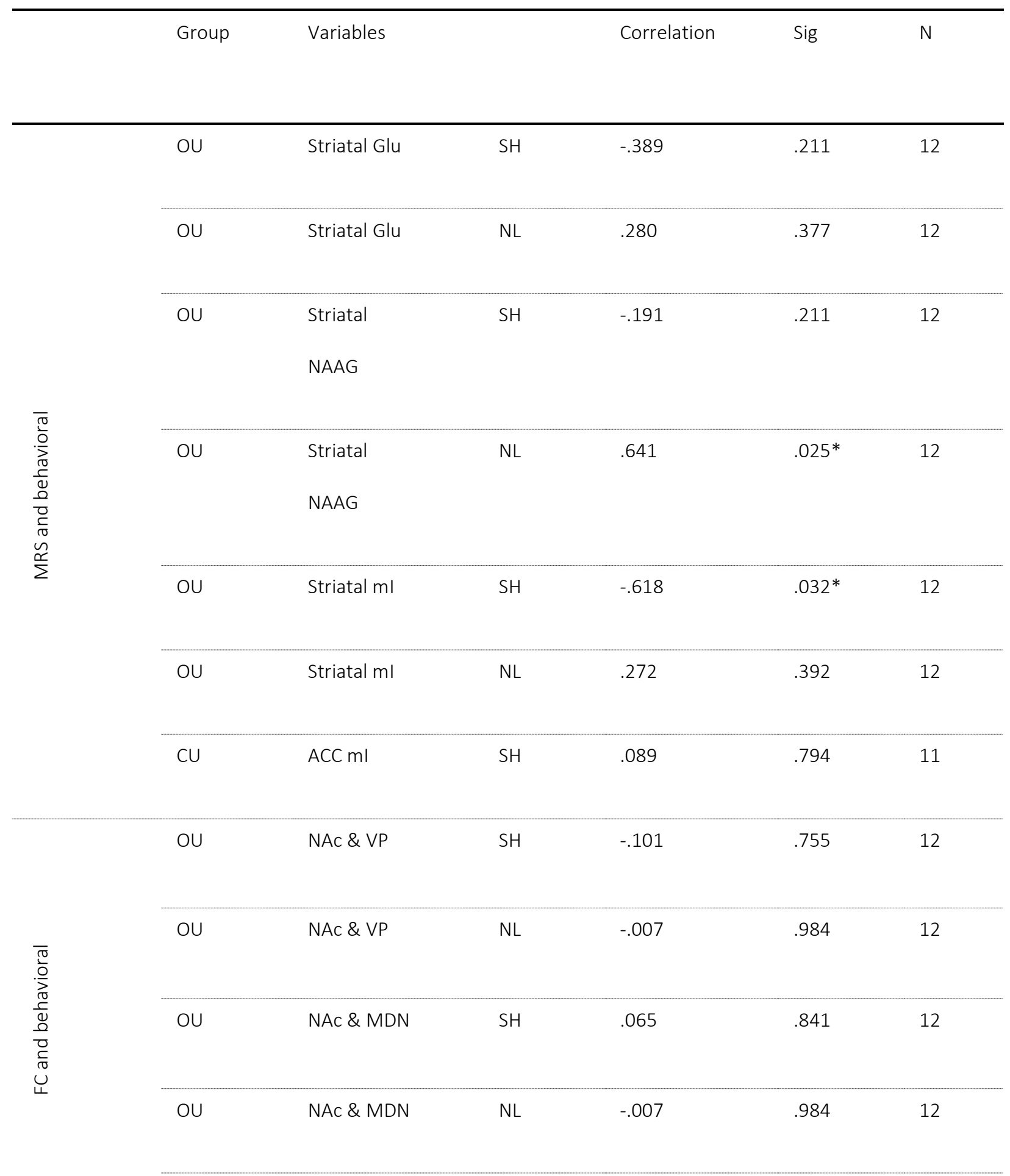


Reduced responsiveness of the reward system in chronic cannabis users

\begin{tabular}{|c|c|c|c|c|c|}
\hline OU & VP \& MDN & $\mathrm{SH}$ & .470 & .123 & 12 \\
\hline OU & VP \& MDN & $\mathrm{NL}$ & -.224 & .484 & 12 \\
\hline $\mathrm{OU}$ & MDN \& MC & $\mathrm{SH}$ & .182 & .572 & 12 \\
\hline OU & MDN \& MC & $\mathrm{NL}$ & -.289 & .363 & 12 \\
\hline OU & $N A c \& M C$ & $\mathrm{SH}$ & -.334 & .289 & 12 \\
\hline OU & $N A C \& M C$ & $\mathrm{NL}$ & .048 & .882 & 12 \\
\hline
\end{tabular}

*Significant $P$ value

$\mathrm{Glu}=$ glutamate; $\mathrm{NAAG}=$ total $\mathrm{N}$-acetyl-aspartate; $\mathrm{ml}=$ myoinositol; $\mathrm{ACC}=$ anterior cingulate cortex; Nac = nucleus accumbens; $\mathrm{VP}=$ ventral pallidum; $\mathrm{MDN}=$ medial dorsal nucleus; $\mathrm{MC}=$ midcingulate area; $\mathrm{SH}=$ subjective high; $\mathrm{NL=}$ number of lapses on the psychomotor vigilance task, FC= functional connectivity; 


\section{Chapter 3}

Table S8. Significant correlations between functional connectivity and average change scores of the variables of interest.

\begin{tabular}{|c|c|c|c|c|c|c|c|c|c|c|}
\hline \multirow[t]{2}{*}{ Correlation } & \multirow[t]{2}{*}{$+/-$} & \multirow[b]{2}{*}{ Cluster } & \multicolumn{2}{|c|}{$\begin{array}{l}R \text { or } \\
L\end{array}$} & \multirow[t]{2}{*}{ k } & \multirow[t]{2}{*}{$x$} & \multirow[t]{2}{*}{ y } & \multirow[t]{2}{*}{ z } & \multirow{2}{*}{$\begin{array}{l}\text { P value } \\
\text { FWE } \\
\text { cluster } \\
\text { corrected }\end{array}$} & \multirow[t]{2}{*}{ Pearson's R } \\
\hline & & & & BA & & & & & & \\
\hline \multirow[t]{2}{*}{ VAS } & + & Middle frontal gyrus & L & 44 & 47 & -44 & 28 & 36 & 0.000 & $\begin{array}{l}r=.761, \\
n=12, P \\
=.004\end{array}$ \\
\hline & & Medial frontal gyrus & $\mathrm{R}$ & 10 & 19 & 12 & 58 & 10 & 0.004 & $\begin{array}{l}r=.708, \\
n=12, P \\
=.010\end{array}$ \\
\hline \multirow[t]{3}{*}{ Number of lapses } & + & Middle frontal gyrus & $R$ & 9 & 26 & 34 & 40 & 46 & 0.000 & $\begin{array}{l}r=.843, \\
n=12, P \\
=.001\end{array}$ \\
\hline & & Superior frontal gyrus & $\mathrm{R}$ & & 49 & 20 & 22 & 48 & 0.000 & $\begin{array}{l}r=.641, \\
n=12, P \\
=.025\end{array}$ \\
\hline & & $\begin{array}{l}\text { Medial orbitofrontal } \\
\text { cortex }\end{array}$ & L & 11 & 14 & -10 & 34 & -12 & 0.011 & $\begin{array}{l}r=.739, \\
n=12, P \\
=.009\end{array}$ \\
\hline
\end{tabular}

MNI coordinates of peak voxels for each cluster are given. 


\section{Reduced responsiveness of the reward system in chronic cannabis users}

\section{References}

1. Theunissen EL, Kauert GF, Toennes SW, et al. Neurophysiological functioning of occasional and heavy cannabis users during THC intoxication. Psychopharmacology (Berl). 2012;220:341-350.

2. Ramaekers JG, van Wel JH, Spronk DB, et al. Cannabis and tolerance: acute drug impairment as a function of cannabis use history. Scientific Reports. 2016;6.

3. Loh S, Lamond N, Dorrian J, Roach G, Dawson D. The validity of psychomotor vigilance tasks of less than 10-minute duration. Behavior research methods, instruments, \& computers : a journal of the Psychonomic Society, Inc. 2004;36:339346.

4. Mason NL, Theunissen EL, Hutten NR, et al. Cannabis induced increase in striatal glutamate associated with loss of functional corticostriatal connectivity. European Neuropsychopharmacology. 2018;29:247-256.

5. Frahm J, Bruhn H, Gyngell ML, Merboldt KD, Hanicke W, Sauter R. Localized high-resolution proton NMR spectroscopy using stimulated echoes: initial applications to human brain in vivo. Magnetic resonance in medicine. 1989;9:79-93.

6. Tkac I, Starcuk Z, Choi IY, Gruetter R. In vivo $1 \mathrm{H}$ NMR spectroscopy of rat brain at 1 ms echo time. Magnetic resonance in medicine. 1999;41:649-656.

7. Klose U. In vivo proton spectroscopy in presence of eddy currents. Magnetic resonance in medicine. 1990;14:26-30.

8. Barker PB, Soher BJ, Blackband SJ, Chatham JC, Mathews VP, Bryan RN. Quantitation of proton NMR spectra of the human brain using tissue water as an internal concentration reference. NMR in biomedicine. 1993;6:89-94.

9. Soher BJ, Hurd RE, Sailasuta N, Barker PB. Quantitation of automated single-voxel proton MRS using cerebral water as an internal reference. Magnetic resonance in medicine. 1996;36:335-339.

10. Smith SA, Levante TO, Meier BH, Ernst RR. Computer Simulations in Magnetic Resonance. An Object-Oriented Programming Approach. Journal of Magnetic Resonance. 1994;106:75-105.

11. Govindaraju V, Young K, Maudsley AA. Proton NMR chemical shifts and coupling constants for brain metabolites. NMR in biomedicine. 2000;13:129-153.

12. Penner J, Bartha R. Semi-LASER 1 H MR spectroscopy at 7 Tesla in human brain: Metabolite quantification incorporating subject-specific macromolecule removal. Magnetic resonance in medicine. 2014.

13. Marques JP, Kober T, Krueger G, van der Zwaag W, Van de Moortele PF, Gruetter R. MP2RAGE, a self bias-field corrected sequence for improved segmentation and T1-mapping at high field. Neurolmage. 2010;49:1271-1281.

14. Zhang Y, Brady M, Smith S. Segmentation of brain MR images through a hidden Markov random field model and the expectation-maximization algorithm. IEEE transactions on medical imaging. 2001;20:45-57.

15. Chao-Gan Y, Yu-Feng Z. DPARSF: A MATLAB Toolbox for "Pipeline" Data Analysis of Resting-State fMRI. Frontiers in systems neuroscience. 2010;4:13.

16. Rosner B. Fundamentals of Biostatistics. 6 ed. Belmont, CA: Thomson-Brooks/Cole; 2006.

17. Ramaekers J, Evers E, Theunissen E, Kuypers K, Goulas A, Stiers P. Methylphenidate reduces functional connectivity of nucleus accumbens in brain reward circuit. Psychopharmacology (Berl). 2013;229:219-226.

18. Ramaekers JG, van Wel JH, Spronk D, et al. Cannabis and cocaine decrease cognitive impulse control and functional corticostriatal connectivity in drug users with low activity DBH genotypes. Brain Imaging and Behavior. 2016;10:12541263.

19. Filbey FM, Aslan S, Lu H, Peng S-L. Residual effects of THC via novel measures of brain perfusion and metabolism in a large group of chronic cannabis users. Neuropsychopharmacology. 2018;43:700.

20. Di Martino A, Scheres A, Margulies DS, et al. Functional connectivity of human striatum: a resting state FMRI study. Cerebral cortex (New York, NY: 1991). 2008;18:2735-2747.

21. Kelly C, de Zubicaray G, Di Martino A, et al. L-dopa modulates functional connectivity in striatal cognitive and motor networks: a double-blind placebo-controlled study. The Journal of neuroscience : the official journal of the Society for Neuroscience. 2009;29:7364-7378. 


\section{Chapter 4}

\section{Me, Myself, Bye: regional alterations in glutamate and the experience of ego dissolution with psilocybin}

Mason, N.L., Kuypers, K.P.C., Muller, F., Reckweg, J., Tse, D.H.Y., Toennes, S.W., Hutten, N.R.P.W., Jansen, J.F.A., Stiers, P., Feilding, A., \& Ramaekers, J.G.

There is growing interest in the therapeutic utility of psychedelic substances, like psilocybin, for disorders characterized by distortions of the self-experience, like depression. Accumulating preclinical evidence emphasizes the role of the glutamate system in the acute action of the drug on brain and behavior; however this has never been tested in humans. Following a double-blind, placebo-controlled, parallel group design, we utilized an ultra-high field multimodal brain imaging approach and demonstrated that psilocybin $(0.17 \mathrm{mg} / \mathrm{kg})$ induced region-dependent alterations in glutamate, which predicted distortions in the subjective experience of one's self (ego dissolution). Whereas higher levels of medial prefrontal cortical glutamate were associated with negatively experienced ego dissolution, lower levels in hippocampal glutamate were associated with positively experienced ego dissolution. Such findings provide further insights into the underlying neurobiological mechanisms of the psychedelic, as well as the baseline, state. Importantly, they may also provide a neurochemical basis for therapeutic effects as witnessed in ongoing clinical trials.

Neuropsychopharmacology, 2020. 


\section{Chapter 4}

\section{Introduction}

Psychedelics are a class of psychoactive substances which induce profoundly altered states of consciousness, including transient and dose-dependent distortions in the subjective experience of one's self ${ }^{1}$. Termed ego dissolution ${ }^{2}$, this phenomenon is characterized by the reduction in the self-referential awareness that defines normal waking consciousness, ultimately disrupting selfworld boundaries and increasing feelings of unity with others' and one's surroundings ${ }^{3}$. Importantly, there is a renewed interest in the use of these substances in the treatment of various psychiatric conditions characterized by distortions of the self-experience ${ }^{4,5}$. Recent clinical studies have suggested that these substances can increase well-being ${ }^{4,6-11}$ and provide therapeutic relief for those suffering from anxiety, depression, and addiction ${ }^{4,9,12-15}$.

Converging evidence suggests that classical psychedelics, such as lysergic acid diethylamide (LSD), psilocybin, and dimethyltryptamine (DMT), stimulate serotonin (5-HT2A) receptors located on cortical pyramidal neurons, which is the suggested primary mechanism of action for their hallucinogenic effect ${ }^{16-22}$. Nevertheless, accumulating evidence from preclinical studies also emphasizes the role of the glutamate system in $5-\mathrm{HT}_{2 \mathrm{~A}}$ receptor-mediated effects on brain function ${ }^{19,23,24}$ and behavior ${ }^{17}$. Specifically, it has been suggested that activation of $5-\mathrm{HT}_{2 \mathrm{~A}}$ receptors leads to a glutamate-dependent increase in activity of pyramidal neurons in the prefrontal cortex ${ }^{18,19,25,26}$, subsequently modulating prefrontal network activity ${ }^{16}$. Furthermore, the increase in extracellular glutamate has been suggested to activate AMPA receptors located on the same neurons, increasing expression of brain-derived neurotrophic factor (BDNF) $16,27,28$, a protein implicated in neuronal survival and growth, and decreased in pathological populations ${ }^{29}$. Taken together, it has been suggested that $5-\mathrm{HT}_{2 \mathrm{~A}}$ receptor-mediated glutamate release is the final common pathway for the acute actions of psychedelics, and a potential underlying mechanism of therapeutic effects ${ }^{16}$. However, no study has investigated the acute effect of a psychedelic on brain glutamate levels in humans, and its relationship with established psychedelic-induced alterations on brain function and behavior.

The present study was designed to establish the contribution of glutamate to the psychedelic state by using ultra-high field (7T) proton Magnetic Resonance Spectroscopy (MRS) that allows in vivo assessment of glutamate in designated brain areas. First, we assessed the acute influence of the classic psychedelic, psilocybin, on glutamate concentration levels in the human brain. Then we assessed the association between glutamate levels, and key features of the psychedelic state, e.g. the experience of ego dissolution, and disrupted resting state network (RSN) functional connectivity (FC). It has been repeatedly found that LSD, DMT and psilocybin decrease within-network connectivity in several RSNs while increasing connectivity across such networks ${ }^{30}$ 36. Affected RSNs include the default mode network (DMN), an interconnected group of brain 
structures including the medial prefrontal cortex (mPFC), posterior cingulate cortex, and inferior parietal lobule 37,38 . Importantly, the DMN in particular has become closely associated with selfreferential mental activity ${ }^{37,39}$, and psychedelic-induced alterations in DMN function have been repeatedly implicated in the experience of ego-dissolution ${ }^{33,40-42}$.

Relative glutamate concentrations were quantified in the MPFC and the hippocampus. These areas were chosen based on previous anatomical, functional, and behavioral evidence implicating them as potential key regions in modulating the psychedelic experience. Specifically, both areas contain a high density of $5-\mathrm{HT}_{2 \mathrm{~A}}$ receptors ${ }^{43}$, which is among the most abundant $5-\mathrm{HT}$ receptor expressed in these regions ${ }^{44,45}$. Functionally, pre-clinical studies have shown increased glutamate concentrations in the mPFC after $5-\mathrm{HT}_{2 \mathrm{~A}}$ agonism ${ }^{18,19,25,26}$, whereas in humans increased glucose, indicative of higher metabolic demands due to increased cell excitability, has been found in frontal and temporal regions after psilocybin ${ }^{46}$. Finally, both areas anatomically overlap with the DMN, with the mPFC recognized as a major hub37,38, and a decoupling between the DMN and medial temporal lobe (MTL; especially hippocampal regions) hypothesized to be a key mechanism in the experience of ego-dissolution ${ }^{47-49}$.

Resting state functional magnetic resonance imaging (rsfMRI) was used to assess RSN FC. Within-network FC assessment followed the approaches of previous studies to allow for comparability $^{32}$, and subjective state was characterized via a well-established altered states of consciousness questionnaire ${ }^{2,50}$, and a validated questionnaire to assess ego dissolution ${ }^{1}$. As MRS captures a range of brain metabolites, we also performed an exploratory analysis to assess whether psilocybin affected other metabolites of interest, including $\mathrm{\gamma}$-aminobutyric acid $(\mathrm{GABA})^{17}$, and markers of neuronal integrity and glial activation, including n-acetyl-aspartate (NAA), and myo-inositol (ml).

\section{Materials and Methods}

A detailed description of the experimental procedure, image acquisition, MRS quantification, and rsfMRI analysis is provided in the Supplementary Methods, and briefly summarized here.

The present study employed a randomized, placebo-controlled, double-blind, parallel group design. Sixty healthy participants, with previous experience with a psychedelic drug but not within the past 3 months, were allocated to a treatment condition $(0.17 \mathrm{mg} / \mathrm{kg}$ psilocybin or placebo, p.o.). Groups were matched for age, sex, and education level.

This study was conducted according to the code of ethics on human experimentation established by the declaration of Helsinki (1964) and amended in Fortaleza (Brazil, October 2013) and in accordance with the Medical Research Involving Human Subjects Act (WMO) and was approved by the Academic Hospital and University's Medical Ethics committee. All participants 
were fully informed of all procedures, possible adverse reactions, legal rights and responsibilities, expected benefits, and their right for voluntary termination without consequences.

\section{Image acquisition}

Participants underwent structural MRI (50 minutes post treatment), single-voxel proton MRS in the mPFC (65 minutes post) and hippocampus (95 minutes post), and fMRI (102 minutes post), during peak subjective drug effects. Images were acquired on a MAGNETOM 7T MR scanner.

Spectroscopic voxels were placed by a trained operator at the mPFC (voxel size $=25 \times 20 x$ $17 \mathrm{~mm}^{3}$ ) and the right hippocampus (voxel size $=37 \times 15 \times 15 \mathrm{~mm}^{3}$ ). Spectra were acquired with the stimulated echo acquisition mode $(S T E A M)^{51}$ sequence (TE $=6.0 \mathrm{~ms}, \mathrm{TR}=5.0 \mathrm{~s}, 64$ averages). Outcome measures for MRS were concentration ratios of glutamate, GABA, NAA, and ml, to total Creatine ( $\mathrm{tCr}$, Creatine + Phospho-Creatine), because using $\mathrm{tCr}$ as the internal reference inherently corrects for variabilities caused by transmit or receive RF inhomogeneity, magnetic field drift, and CSF inclusion in the voxel ${ }^{52}$.

Additionally, 258 whole-brain EPI volumes were acquired at rest (TR=1400 ms; TE= 21 ms; field of view $=198 \mathrm{~mm}$; flip angle $=60^{\circ}$; oblique acquisition orientation; interleaved slice acquisition; 72 slices; slice thickness=1.5 mm; voxel size=1.5×1.5×1.5 mm). During scanning, participants were shown a black cross on a white background, and instructed to focus on the cross while clearing their mind and laying as still as possible.

\section{Processing of imaging data}

Spectroscopy data was analyzed with LCModel version 6.3-1H.

Resting state data was processed and analysed using the CONN toolbox $18 . b^{53}$.All volumes were realigned, unwarped, segmented into grey and white matter and cerebrospinal fluid, normalised into a standard stereotactic space (Montreal Neurological Institute) and smoothed with a $6 \mathrm{~mm}$ full width at half maximum Gaussian kernel.

Independent component analysis (ICA) was performed using group-ICA procedures implemented in the CONN toolbox following previously described methods ${ }^{54}$. Independent components were restricted to 20 in order to allow comparisons with 10 established RSNs ${ }^{55}$ and previous studies on psilocybin ${ }^{42}$ and LSD 32,33 .

\section{Subjective state}

The 5 Dimensions of Altered States of Consciousness (5D-ASC) scale ${ }^{50}$ and the Ego Dissolution Inventory $(E D I)^{1}$ were administered 360 minutes after drug administration, as retrospective measures of drug effects. 


\section{Pharmacokinetic measures.}

Venous blood samples were collected after treatment administration (at 80, 150, and 360 minutes) in order to assess concentrations of psilocin, the main metabolite of psilocybin.

\section{Statistical analysis}

Statistical analysis of metabolite concentration levels and questionnaire responses were conducted in IBM SPSS Statistics 24 using nonparametric Mann-Whitney U tests.

For the assessment of within-network FC, the unthresholded, binarized ICA component images were compared between placebo and drug conditions (two-sample $t$-test). Parametric statistics were used (voxel threshold $p<0.001$ uncorrected, cluster threshold $p<0.05$ cluster-size, false discovery rate (FDR) corrected, two-sided).

For the assessment of between-network FC, unthresholded, binarized maps of RSNs obtained from the ICA analysis were imported as ROIs and the weighted sums of the time series were extracted. Time courses between all RSNs were then compared for both conditions using bivariate correlations. The resulting correlation coefficients were compared between placebo and drug conditions (two-sample $t$-test). Results were corrected for multiple comparisons using FDR.

Canonical correlations ${ }^{56}$ were conducted to evaluate the association between psilocybininduced changes in (i) relative glutamate concentration levels in the MPFC and hippocampus, (ii) ratings of ego dissolution, including 2 dimensions of the 5D-ASC (oceanic boundlessness and anxious ego dissolution) and scores on the EDI, and (iii) within-network resting state FC, using extracted connectivity strength (beta) values. Variables were separated into two sets; set 1 included biological variables as predictors $[(i)$ and $(i i i)]$ and set 2 included the subjective variables as criterion (ii). An iterative imputation approach was performed to fill in missing data points, when applicable.

The alpha criterion of significance of all tests was assumed at $p<0.05$

\section{Results}

\section{Demographic variables and psilocin concentration levels}

The psilocybin group $(n=30)$ and the placebo group $(n=30)$ did not differ in respect to demographic variables(Table S1).

Mean (S.E.) concentrations of psilocin in serum are given in Table S2. Concentrations reached a peak 80 minutes postdrug administration (15.61 $\pm 1.66 \mathrm{ng} / \mathrm{mL})$, and then began to fall (360 minutes post $4.85 \pm 0.54 \mathrm{ng} / \mathrm{mL}$ ). The measured concentrations are in accordance with the applied oral dose ${ }^{57}$. 


\section{Acute effect of psilocybin on subjective state}

Administration of psilocybin was associated with increased ratings on all (sub)dimensions of the 5D-ASC $(U=13.5-225 ; p \leq 0.001$ effect size $=.43-.84$, Figure $1 \mathrm{~A} ; \mathrm{S} 1)$, and on the ego dissolution inventory (EDI; $U=91.5, p<0.001$, effect size=0.67, Figure $1 B$ ).

A.

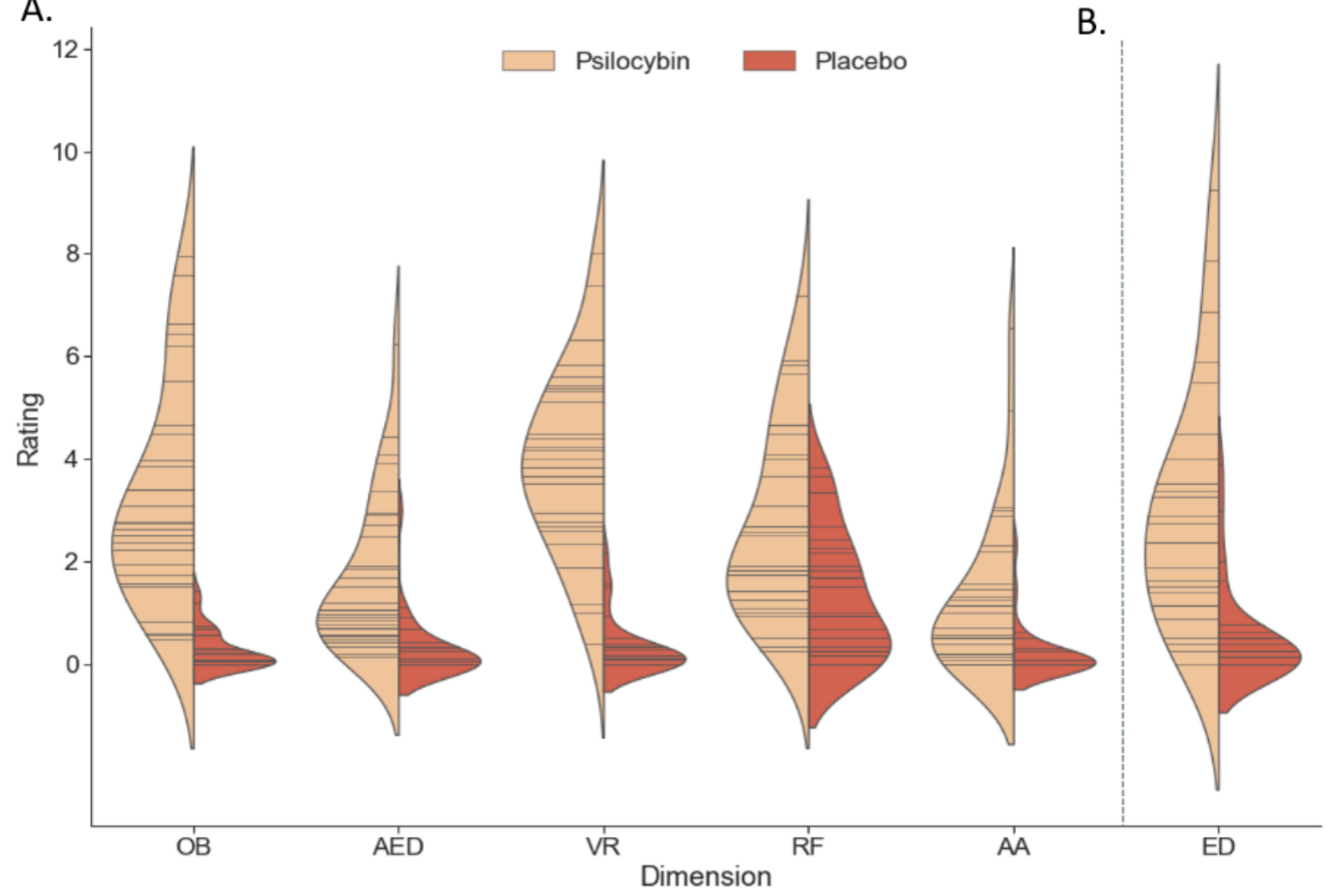

Figure 1. Violin plots displaying reported scores on the 5 main dimensions of the 5D-ASC (A), ratings on the ego dissolution inventory (B) for each treatment group. Each stick in the violin indicates a data point, whereas the density is scaled to the relative count across all bins.

\section{MRS results}

Spectral quality for each treatment condition is reported in Table 1. Overview of data points that did not meet the data quality criteria check can be found in Table S6. 
Table 1. Mean (SD) spectral quality per group. Only data points with an $S N R>10, F W H M<0.1$, and a CRLB $<20 \%$ were included in the final analysis. $N$ refers to the number of data points that met the criteria, per metabolite, per group, and were included in the analysis.

\begin{tabular}{|c|c|c|c|c|}
\hline Parameter & Psilocybin & Placebo & $t$ value & $P$ value \\
\hline \multicolumn{5}{|l|}{ Medial prefrontal cortex } \\
\hline \multicolumn{5}{|l|}{$\begin{array}{l}\text { Relative Cramer-Rao lower bound } \\
(\%) ; \mathrm{n}\end{array}$} \\
\hline Glutamate & $2.62(0.57) ; 24$ & $2.71(0.60) ; 28$ & -0.54 & 0.58 \\
\hline$G A B A$ & $13.33(3.52) ; 15$ & 14.65 (3.33); 17 & -1.08 & 0.29 \\
\hline$N A A+N A A G$ & $2.37(0.49) ; 24$ & $2.32(0.55) ; 28$ & 0.36 & 0.71 \\
\hline Myoinositol & $4.33(2.14) ; 24$ & $4.11(1.42) ; 28$ & 0.45 & 0.65 \\
\hline Signal to noise ratio & $36.87(4.77)$ & $37.96(6.52)$ & -0.67 & 0.50 \\
\hline $\begin{array}{l}\text { Full-width at half-maximum peak } \\
\text { height }\end{array}$ & $0.04(.01)$ & $0.04(.01)$ & -.307 & 0.76 \\
\hline
\end{tabular}

Hippocampus

Relative Cramer-Rao lower bound

(\%);

n

$\begin{array}{llccc}\text { Glutamate } & 5.85(2.32) ; 21 & 5.00(1.73) ; 25 & 1.43 & 0.16 \\ \text { GABA } & 16.60(1.67) ; 5 & 14.50(3.07) ; 8 & 1.39 & 0.19 \\ \text { NAA + NAAG } & 2.90(0.77) ; 21 & 2.96(0.93) ; 25 & -.22 & 0.83 \\ \text { Myoinositol } & 4.70(1.26) ; 20 & 4.62(3.12) ; 24 & 0.10 & 0.92 \\ \text { noise ratio } & 20.57(5.61) & 21.16(6.86) & -.31 & 0.75 \\ & & & & 0.08\end{array}$


Medial prefrontal cortex. As hypothesized, glutamate/total creatine (Glutamate) in the mPFC was higher after psilocybin, compared to placebo (mean \pm S.E.; psilocybin: $1.23 \pm 0.02$; placebo: 1.14 $\pm 0.02, U=200.50, p=0.01$, effect size $=0.80$, Figure $2 \mathrm{~A}$ ).

In addition, tNAA/total creatine (psilocybin: $1.41 \pm 0.03$; placebo: $1.31 \pm 0.02, U=210.0, p=$ 0.02 , effect size $=0.72$, Figure $2 B$ ), and GABA/total creatine (psilocybin: $0.17 \pm 0.01$; placebo: 0.14 $\pm 0.01, U=66.0, p=0.01$, effect size $=0.99$, Figure $2 \mathrm{D}$ ) were higher after psilocybin, compared to placebo.

Hippocampus. In contrast, glutamate in the hippocampus (psilocybin: $0.77 \pm 0.03$; placebo: $0.88 \pm$ 0.03, $U=163.50, p=0.03$, effect size $=0.69$, Figure $2 \mathrm{C}$ ) was lower after psilocybin, compared to placebo.

No other significant differences were seen between groups in regards to relative concentrations of GABA, tNAA, ml, or total creatine concentration. See Table S3 for means of all investigated metabolite concentration, additional metabolites acquired in the spectra, and Figure S2 for representative spectra and voxel placement.

\section{Resting state networks}

After quality control, the final sample consisted of 22 participants in the psilocybin group and 26 in the placebo group. There were no significant differences between groups in regards to head motion parameters. See supplementary for exclusion criteria and assessed differences between groups.

Independent component analysis. There was a good agreement between most of the components identified in our analysis and the templates provided by Smith et al. (Smith et al., 2009). We were able to identify the visual networks $1-3$ ( $r=0.80, r=0.73$ and $r=0.64$, respectively), the cerebellar network ( $r=0.38)$, the auditory network $(r=0.43)$, the executive control network $(r=0.58)$ and the frontoparietal networks $1(r=0.50)$ and $2(r=0.47)$. In contrast, we were not able to assign a single component to the DMN and the sensorimotor network, as these networks were split up in subcomponents, as already observed in multiple studies ${ }^{58-60}$. The DMN consisted of two components (anterior DMN: $r=0.34$ and posterior DMN: $r=0.52$ ) and the sensorimotor network consisted of three components (somatosensory network: $r=0.40$, lateral motor network: $r=0.32$, medial motor network: $r=0.24)$. In order to allow a comprehensive exploration, we decided to include all of these components in further analysis. For this purpose, the respective sub-components were labelled according to common terminology $y^{60}$ but in deviation from previous work ${ }^{55}$ (i.e. the components were labelled as anterior and posterior DMN, medial and lateral motor network and 
somatosensory network). The remaining seven components reflected noise or networks that were not relevant for this analysis.

A.

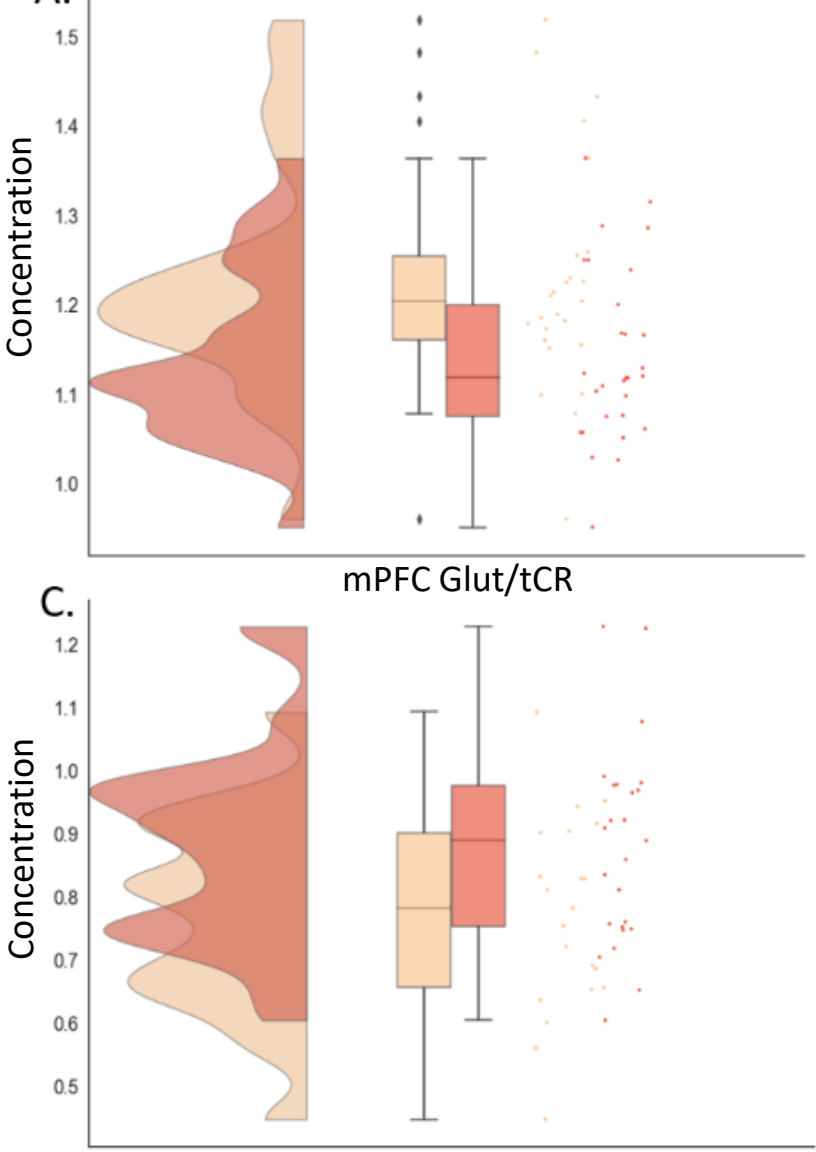

Hippocampal Glut/tCR
B.

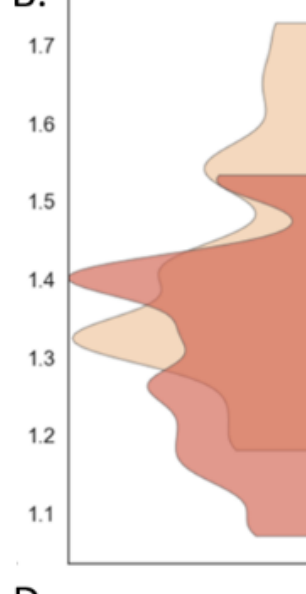

D.

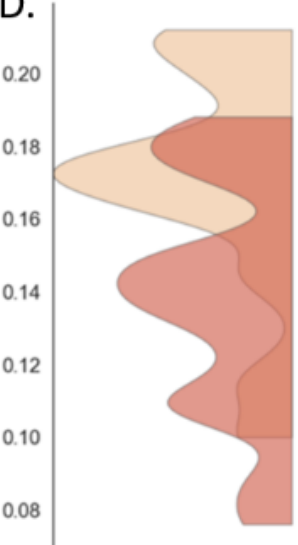

Psilocybin

Placebo

mPFC NAA/tCR

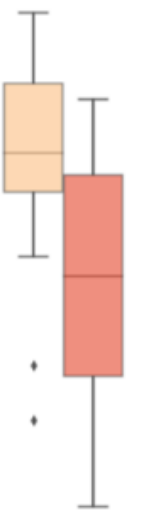

mPFC GABA/tCR

Figure 2. Raincloud plots displaying metabolite concentrations in the MPFC and the hippocampus, which demonstrated significant differences between treatment groups. A. glutamate in the MPFC, B. NAA in the MPFC, C. glutamate in the hippocampus, D. GABA in the MPFC. The plot consists of a probability density plot, a boxplot, and raw data points. In the boxplot, the line dividing the box represents the median of the data, the ends represent the upper/lower quartiles, and the extreme lines represent the highest and lowest values excluding outliers. The code for raincloud plot visualization has been adapted from Allen, et al. ${ }^{99}$. 


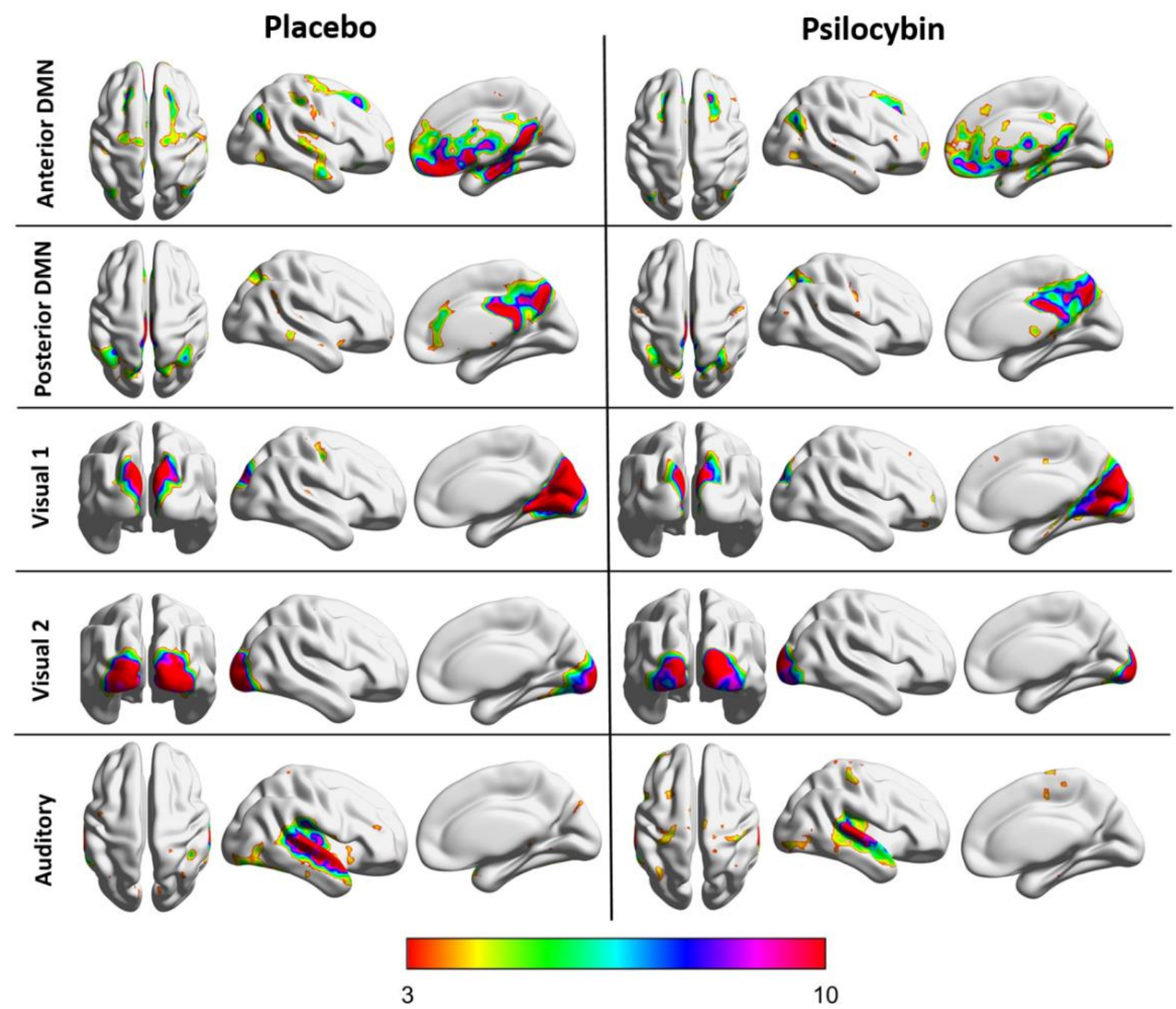

Figure 3. Resting state networks that demonstrated significant differences in within-network functional connectivity, for each group (placebo and psilocybin).

Within-network connectivity. Within the respective network, significantly less coactivation under the drug condition relative to placebo was found in visual network 1 and 2, both subcomponents of the DMN (anterior and posterior), and the auditory network (Figure 3; Table S4).

Between-network connectivity. Widespread increases in between-network FC were observed under psilocybin compared to placebo. Except the lateral motor network, all investigated networks were affected to some extent (Table S5).

\section{Relationship between psilocybin-induced changes in brain and behavior.}

A canonical correlation analysis was conducted using the four biological variables as predictors of the three ego dissolution variables, to evaluate the multivariate shared relationship between the two variable sets. The analysis yielded three functions with squared canonical correlations $\left(R_{c}{ }^{2}\right)$ of 
$.363, .282$, and .253 for each successive function. The full model across all functions was statistically significant $F(12,61.14)=2.47, p=.008)$, explaining $65.9 \%$ of the variance.

Given the $R_{c}{ }^{2}$ effects for each function, the first two functions were significant ( $p=.008$ and $\mathrm{p}=.016$, respectively) and considered noteworthy in the context of this study, with function 1 explaining $36.3 \%$ of the variance, and function 2 explaining $28.2 \%$ of the variance.

Table 2 presents the standardized canonical function coefficients, the structure coefficients $\left(r_{s}\right)$, and the squared structure coefficients $\left(r_{s}{ }^{2}\right)$ for functions 1 and 2 , as well as the communalities $\left(h^{2}\right)$ across the two functions for each variable. Function 1 indicated that the dominant contributor was anxious ego dissolution (AED), with oceanic boundlessness (OB) making a secondary contribution. In regards to predictors, mPFC glutamate was the dominant predictor, with anterior DMN FC making a secondary contributions. These results suggest that the strongest predictor of negatively experienced ego dissolution (i.e. AED) was the increase in mPFC glutamate.

Function 2 indicated that the dominant contributor was ratings on the ego dissolution inventory (EDI), with OB as a secondary contribution. As for the predictors, hippocampal glutamate was the strongest predictor, with posterior DMN FC making a secondary contribution. These results suggest that the strongest predictor of positively experienced ego dissolution was the decrease in hippocampal glutamate. 


\section{Chapter 4}

Table 2. Canonical solution for biological variables predicting ego dissolution for Functions 1 and 2. $r_{s}$ greater than $/ .45 /$ and $h^{2}$ greater than $45 \%$ are underlined and deemed valuable contributors.

\begin{tabular}{|c|c|c|c|c|c|c|c|}
\hline & Functio & & & Functio & & & \\
\hline Variable & Coef & $r_{s}$ & $r_{s}^{2}(\%)$ & Coef & $r_{s}$ & $r_{s}^{2}(\%)$ & $h^{2}(\%$, \\
\hline Oceanic boundlessness & -1.439 & $\underline{-.568}$ & 32.2 & .322 & -.689 & 47.5 & $\underline{79.7}$ \\
\hline Anxious ego dissolution & -.810 & $\underline{-.681}$ & 46.4 & .727 & -.365 & 13.3 & $\underline{59.7}$ \\
\hline Ego dissolution inventory & 1.347 & -.274 & 7.5 & -1.389 & $\underline{-.956}$ & 91.4 & $\underline{98.9}$ \\
\hline$R c^{2}$ & & & 36.3 & & & 28.2 & \\
\hline Glutamate/tCr hippocampus & -.648 & .113 & 1.3 & 1.102 & .990 & 98.0 & $\underline{99.3}$ \\
\hline Glutamate/tCr mPFC & -.724 & $\underline{-.630}$ & 40.0 & .036 & -.047 & 0.2 & 40.2 \\
\hline Anterior DMN & .853 & .520 & 27.0 & -.166 & .400 & 16.0 & 43.0 \\
\hline Posterior DMN & .551 & .313 & 9.7 & -.047 & .484 & 23.4 & 33.1 \\
\hline
\end{tabular}

Coef = standardized canonical function coefficients; $r_{s}=$ structure coefficients; $r_{s}^{2}=$ squared structure coefficient; $h^{2}=$ communality coefficient; $R_{c}^{2}=$ squared canonical coefficient

\section{Discussion}

The present study demonstrates the first attempt to assess the acute effects of psilocybin on glutamate levels in key areas of the human brain, which may play a major role in the actions of serotonergic psychedelics. Using an ultra-high field multimodal MRI approach, we demonstrated that, compared to placebo, psilocybin-induced region-dependent alterations in neurometabolite concentrations. Specifically, participants who received psilocybin demonstrated higher relative glutamate concentration levels in the MPFC, and lower relative glutamate concentration levels in the hippocampus. Analyses indicated that region-dependent alterations in glutamate also correlated with different dimensions of ego dissolution. Whereas changes in mPFC glutamate were found to be the strongest predictor of negatively experienced ego dissolution, changes in hippocampal glutamate were found to be the strongest predictor of positively experienced ego dissolution.

Previous studies have demonstrated that the mPFC is highly enriched with $5-\mathrm{HT}_{2 \mathrm{~A}}$ receptors located primarily on layer $V$ pyramidal neurons ${ }^{61}$, and modulate excitatory transmission in cortical circuits ${ }^{43,62,63}$. Preclinical studies have demonstrated that activation of such receptors 
via serotonergic psychedelics results in a predominantly excitatory response ${ }^{18,64}$ via an increase in glutamate release, as observed in humans for the first time in this study. A glutamatergic increase in this area is also in accordance with human functional imaging studies which have demonstrated a hyperfrontal regional cerebral blood flow (CBF) pattern after psilocybin ${ }^{46,65}$, and similar $5-\mathrm{HT}_{2 \mathrm{~A}}$ agonist psychedelics ${ }^{66,67}$. However, we also found that psilocybin administration was associated with higher levels of GABA in this area, results in line with findings that $5-\mathrm{HT}_{2 \mathrm{~A}}$ receptors are also located on GABAergic interneurons ${ }^{17,68}$. Taken together, findings suggest that activation of $5-\mathrm{HT}_{2 \mathrm{~A}}$ receptors in the $\mathrm{mPFC}$ results in both excitation and inhibition of cortical pyramidal cells ${ }^{17}$, potentially resulting in an increased metabolic rate in this area, but not necessarily increased neural input or output.

In contrast to the mPFC, the present study demonstrated that participants who received psilocybin demonstrated lower relative glutamate concentrations in the hippocampus, suggesting that psilocybin decreases glutamate in this area. Such a decrease is in line with data from a recent functional imaging study with psilocybin, demonstrating reduced absolute CBF in the hippocampus compared to placebo69, of which the authors proposed two potential mechanisms. Namely, decrements could be due to agonism of $5-\mathrm{HT}_{2 \mathrm{~A}}$ receptors located on GABAergic interneurons ${ }^{44}$, which can indirectly inhibit pyramidal neurons, decreasing activation in this area. Conversely, it has also been established that, along with the $5-\mathrm{HT}_{2 \mathrm{~A}}$ receptor, psilocin also has a high affinity for the $5-\mathrm{HT}_{1 \mathrm{~A}}$ receptor ${ }^{70,71}$. Referred to as serotonin's principal inhibitory receptor ${ }^{72}$, the $5-\mathrm{HT}_{1 \mathrm{~A}}$ receptors highest density is found in the limbic regions of the brain such as the hippocampus ${ }^{73}$ where it is expressed on neurons that are postsynaptic to the serotonergic input. Thus lower levels in glutamate as seen in this study, as well as regional decreases reflected in others ${ }^{69}$, could be due to activation of post-synaptic inhibitory $5-\mathrm{HT}_{1 \mathrm{~A}}$ receptors. Nevertheless, due to methodological limitations, this study is not able to delineate which mechanism is contributing to the lower levels in glutamate. Further information could have been potentially gained from quantification of GABA in the hippocampus, however we were unable to reliably do so, due to inherent quantification challenges when assessing GABA levels, arising from low brain concentration levels, metabolite signal overlap, and low signal-to-noise in the hippocampus ${ }^{74,75}$. Future studies with sequences developed to specifically quantify GABA in low signal-to-noise areas should make further attempts to do so, given recent research implicating hippocampal GABA in the pathology of disorders that psychedelics are being investigated to treat $^{76}$.

In the current study, psilocybin induced previously established key features of a psychedelic experience: increases in feelings of ego dissolution, and disrupted RSN activity. Psilocybin increased scores on all dimensions of the 5D-ASC ${ }^{16}$, as well as on the EDI ${ }^{1}$. Additionally psilocybin altered within-network FC similarly as has been shown with LSD, including decrements in coactivation within the DMN, visual network 1 , and the auditory network ${ }^{32,33}$. Finally, we 


\section{Chapter 4}

demonstrated higher between-network FC across all networks, which is similar with previous studies assessing the same after psilocybin ${ }^{35,42}$ and LSD 32,33 .

Finally, we assessed the relationship between psilocybin-induced changes in the brain, and the subjective experience of sense of self. Canonical correlations were conducted to predict increases in ratings of $A E D$, the dimension encompassing the loss of autonomy and self-control of thought processes, intentionality, decision making, and spontaneous movements ${ }^{46}$. Our data support the conclusion that increasing levels of mPFC glutamate were the strongest predictor in regards to feelings of AED, with decreasing anterior DMN FC and being a secondary predictor. These findings are in line with previous work, implicating increased frontal metabolism in feelings of AED after psilocybin ${ }^{46}$ and ego pathology in the ketamine model of psychosis ${ }^{77}$. Interestingly, AED-associated changes in mood include paranoia, heightened arousal and attention to the surroundings, and anxiety ${ }^{46}$. A paradoxical effect of serotonergic psychedelics is that acutely they have been found to increase feelings of anxiety 6,78 , whereas clinical trials with psychedelic drugs suggest long-term anxiety relief in patients ${ }^{11,12,14}$. Accordingly, there is a wide range of animal and human pharmacological evidence supporting the role of the glutamatergic system in anxiety ${ }^{79}$, with increases in glutamate in the frontal cortex associated with high versus low state-trait anxiety ${ }^{80}$, and reductions corresponding to anxiety-related symptomatic relief ${ }^{81}$. Taken together, the finding that mPFC glutamate was by far the strongest predictor of increased feelings of anxiety, one could propose that acute psychedelic-induced anxiety may be due to localized glutamateinduced hyperfrontality, whereas long-term reductions could be due to agonist-induced $5-\mathrm{HT}_{2 \mathrm{~A}}$ receptor downregulation in this area ${ }^{72,82}$. Nonetheless, future studies should assess long-term changes in $5-\mathrm{HT}_{2 \mathrm{~A}}$ receptor function in the $\mathrm{MPFC}$, and their relation with subjective effects.

We also assessed the relationship between psilocybin-induced brain changes and feelings of positively experienced ego dissolution, including ratings on the EDI, and scores of OB on the 5DASC. We found that the primary predictor of positively experienced ego dissolution was a decrement in hippocampal glutamate, with a secondary contribution of posterior DMN integrity. Previous work has implicated both the MTL (containing the hippocampus) and DMN circuitry in the neural correlates of the self ${ }^{49}$. Namely, abnormal function of MTL regions have been implicated in psychotic states ${ }^{83,84}$ and feelings of depersonalization ${ }^{85}$ and ego-disturbances ${ }^{86}$. Similarly, studies of drug-induced ego dissolution have found that the decoupling of MTL regions such as the parahippocampus and the DMN correlate positively with feelings of ego dissolution 49,87 , with this decoupling being hypothesized to be one of the main underlying mechanisms of the subjective experience ${ }^{47-49}$. In regards to why this gives rise to ego dissolution, it has been suggested that psychedelic drug-induced decoupling of these regions results in a temporary loss of access of semantic autobiographical information, resulting in a breakdown of one's personal identity ${ }^{87}$. Our data adds to this hypothesis, suggesting that modulations of 
hippocampal glutamate in particular may be a key mediator in the decoupling underlying feelings of (positive) ego dissolution. Interestingly, although the DMN has been the most implicated RSN in this process, Lebedev, et al. ${ }^{49}$ found that increases in ego dissolution correlated with decreased FC between the parahippocampal formation and other major networks, such as the salience, frontoparietal, and sensorimotor network; suggesting a key role in this area in particular, as our data also demonstrates. However future research should further assess the contribution of other areas to this experience, such as the posterior cingulate cortex.

Implications of these findings also extend far beyond understanding the neurobiology of the acute psychedelic experience and drug-induced ego dissolution. There is growing evidence that psychedelics can provide therapeutic relief for individuals suffering from increasingly common and difficult to treat disorders such as depression, anxiety, addiction, and post-traumatic stress disorders $4,9,11,88,89$. Thus understanding the mechanisms by which psychedelics provide symptomatic relief may identify novel therapeutic targets. Interestingly, the degree of ego dissolution has been found to correlate with long-term clinical outcomes ${ }^{90}$ and increases in wellbeing ${ }^{10,91}$. Additionally, a hypothetical (neurobiological) model has been proposed to explain the long-term effects witnessed in clinical trials. It has been suggested that indirect activation of glutamate networks via $5-\mathrm{HT}_{2 \mathrm{~A}}$ receptor agonism increases BDNF, and ultimately enhances neuroplasticity ${ }^{16}$. In line with this, it has been shown in pre-clinical models that psychedelics increase functional and structural neuroplasticity ${ }^{92}$, however evidence in humans is limited, due to restrictions of methodological techniques. Our data provides indirect evidence that psychedelics might have the potential to increase neuroplasticity in the human cortex via increased glutamatergic activity, but not in the hippocampus; findings that are in accordance with previous $5-\mathrm{HT}_{2 \mathrm{~A}}$ receptor activation studies ${ }^{27,93,94}$. Additionally, psilocybin administration was associated with higher levels of MPFC NAA, a compound regarded as a measure of neuronal viability and function, and decreased in disorders associated with regional neuronal loss and disrupted neuronal function ${ }^{95}$.

Of note, compared to previous psychedelic studies, the dose administered was low to moderate ${ }^{96}$, and thus not high enough to induce total ego dissolution. However, the aim of this study was not to assess maximal effects of psilocybin, but rather an effective dose that would induce a relevant psychedelic state that participants could endure in the MRI scanner. Our data demonstrate that the dose was effective, inducing significantly higher levels of both positively and negatively experienced ego dissolution compared to placebo, as well as the other subjective effects representative of a psychedelic state (Figure 1, S1). Furthermore, although BOLD sensitivity is increased by the use of ultra-high magnetic fields, geometric distortions become more prominent, which could have affected our BOLD signal in inferior brain regions ${ }^{97}$, and our scan time was arguably short from a test-retest reliability standpoint ${ }^{98}$. Nevertheless, our results are 
similar to aforementioned studies $32,33,35,42$ who acquired their data at a lower field strength, with varying scanning lengths. Finally, an inherent difficulty of studying substances with such salient subjective effects is maintaining the treatment blind. Thus, it could be suggested that participant recognition of the treatment condition could affect neural and subjective results, emphasizing the importance of active placebo conditions or cross-psychotropic comparisons in future trials.

In conclusion, our data demonstrates that the serotonergic psychedelic, psilocybin, acutely induces region dependent alterations in glutamate that correlate with established behavioral changes during the psychedelic state. Such findings provide further insights into the underlying neurobiological mechanisms of the psychedelic state, and importantly, provide a neurochemical basis for how these substances alter individuals' sense of self, and may be giving rise to therapeutic effects witnessed in ongoing clinical trials.

\section{Funding and disclosure}

This study is part of the Beckley/Maastricht Research Programme. The Beckley Foundation made a financial contribution to the study. The authors report no other relevant funding, and all authors report no potential conflicts of interest.

\section{Acknowledgements}

The authors would like to thank Cees van Leeuwen for medical supervision, Fulya Onus and Sofia Rocha for their assistance with data collection, Professor Robert Leech for analysis advice, all reviewers for their help in improving the manuscript, and all participants for their time and effort.

\section{Author Contributions.}

NM, KK, JG and AF designed the research. NM, JR, and NH performed the research. DT set up and made all of the MRI/MRS possible. JJ provided analysis tools. NM and FM analyzed the data. All authors made a substantial contribution to interpretation of the data and drafting of the manuscript. 
Nour, M. M., Evans, L., Nutt, D. \& Carhart-Harris, R. L. Ego-Dissolution and Psychedelics: Validation of the Ego-Dissolution Inventory (EDI). Frontiers in Human Neuroscience 10, 269, doi:10.3389/fnhum.2016.00269 (2016).

2

Studerus, E., Gamma, A. \& Vollenweider, F. X. Psychometric evaluation of the altered states of consciousness rating scale (OAV). PLOS ONE 5, e12412 (2010).

Nour, M. M. \& Carhart-Harris, R. L. Psychedelics and the science of self-experience. The British journal of psychiatry : the journal of mental science 210, 177-179, doi:10.1192/bjp.bp.116.194738 (2017).

Carhart-Harris, R. L. et al. Psilocybin with psychological support for treatment-resistant depression: an open-label feasibility study. The Lancet Psychiatry 3, 619-627 (2016).

Carhart-Harris, R. L. \& Goodwin, G. M. The Therapeutic Potential of Psychedelic Drugs: Past, Present, and Future. Neuropsychopharmacology 42, 2105-2113, doi:10.1038/npp.2017.84 (2017).

Griffiths, R. R., Richards, W. A., McCann, U. \& Jesse, R. Psilocybin can occasion mystical-type experiences having substantial and sustained personal meaning and spiritual significance. Psychopharmacology 187, 268-283; discussion 284-292, doi:10.1007/s00213-006-0457-5 (2006).

Griffiths, R. R., Richards, W. A., Johnson, M. W., McCann, U. D. \& Jesse, R. Mystical-type experiences occasioned by psilocybin mediate the attribution of personal meaning and spiritual significance 14 months later. Journal of psychopharmacology 22, 621-632 (2008).

Mason, N. L., Mischler, E., Uthaug, M. V. \& Kuypers, K. P. Sub-acute effects of psilocybin on empathy, creative thinking, and subjective well-being. Journal of psychoactive drugs 51, 123-134 (2019).

Johnson, M. W., Garcia-Romeu, A. \& Griffiths, R. R. Long-term follow-up of psilocybin-facilitated smoking cessation. The American journal of drug and alcohol abuse 43, 55-60, doi:10.3109/00952990.2016.1170135 (2017).

Uthaug, M. V. et al. Sub-acute and long-term effects of ayahuasca on affect and cognitive thinking style and their association with ego dissolution. Psychopharmacology, 1-11 (2018).

Griffiths, R. R. et al. Psilocybin produces substantial and sustained decreases in depression and anxiety in patients with life-threatening cancer: A randomized double-blind trial. Journal of psychopharmacology (Oxford, England) 30, 11811197, doi:10.1177/0269881116675513 (2016).

Gasser, P. et al. Safety and efficacy of lysergic acid diethylamide-assisted psychotherapy for anxiety associated with lifethreatening diseases. The Journal of nervous and mental disease 202, 513-520, doi:10.1097/NMD.0000000000000113 (2014).

Palhano-Fontes, F. et al. Rapid antidepressant effects of the psychedelic ayahuasca in treatment-resistant depression: a randomized placebo-controlled trial. Psychological medicine 49, 655-663, doi:10.1017/s0033291718001356 (2019).

Grob, C. S. et al. Pilot study of psilocybin treatment for anxiety in patients with advanced-stage cancer. Archives of general psychiatry 68, 71-78, doi:10.1001/archgenpsychiatry.2010.116 (2011).

Sanches, R. F. et al. Antidepressant Effects of a Single Dose of Ayahuasca in Patients With Recurrent Depression: A SPECT Study. Journal of clinical psychopharmacology 36, 77-81, doi:10.1097/jcp.0000000000000436 (2016).

Vollenweider, F. X. \& Kometer, M. The neurobiology of psychedelic drugs: implications for the treatment of mood disorders. Nature reviews. Neuroscience 11, 642-651, doi:10.1038/nrn2884 (2010).

Nichols, D. E. Psychedelics. Pharmacological reviews 68, 264-355 (2016).

Aghajanian, G. K. \& Marek, G. J. Serotonin induces excitatory postsynaptic potentials in apical dendrites of neocortical pyramidal cells. Neuropharmacology 36, 589-599, doi:10.1016/s0028-3908(97)00051-8 (1997).

Aghajanian, G. K. \& Marek, G. J. Serotonin, via 5-HT2A receptors, increases EPSCs in layer V pyramidal cells of prefrontal cortex by an asynchronous mode of glutamate release. Brain Research 825, 161-171, doi:https://doi.org/10.1016/S00068993(99)01224-X (1999).

Glennon, R. A., Titeler, M. \& McKenney, J. Evidence for 5-HT2 involvement in the mechanism of action of hallucinogenic agents. Life sciences 35, 2505-2511 (1984).

Wing, L. L., Tapson, G. S. \& Geyer, M. A. 5HT-2 mediation of acute behavioral effects of hallucinogens in rats. Psychopharmacology 100, 417-425 (1990).

Sipes, T. \& Geyer, M. DOI disruption of prepulse inhibition of startle in the rat is mediated by 5-HT2A and not by 5-HT2C receptors. Behavioural pharmacology (1995).

Scruggs, J. L., Schmidt, D. \& Deutch, A. Y. The hallucinogen 1-[2,5-dimethoxy-4-iodophenyl]-2-aminopropane (DOI) increases cortical extracellular glutamate levels in rats. Neuroscience letters 346, 137-140, doi:https://doi.org/10.1016/S0304-3940(03)00547-0 (2003).

Scruggs, J. L., Patel, S., Bubser, M. \& Deutch, A. Y. DOI-Induced activation of the cortex: dependence on 5-HT2A heteroceptors on thalamocortical glutamatergic neurons. Journal of Neuroscience 20, 8846-8852 (2000).

Puig, M. V., Celada, P., Díaz-Mataix, L. \& Artigas, F. In vivo modulation of the activity of pyramidal neurons in the rat medial prefrontal cortex by 5-HT2A receptors: relationship to thalamocortical afferents. Cerebral Cortex 13, 870-882 (2003). 


\section{Chapter 4}

Béique, J.-C., Imad, M., Mladenovic, L., Gingrich, J. A. \& Andrade, R. Mechanism of the 5-hydroxytryptamine 2A receptormediated facilitation of synaptic activity in prefrontal cortex. Proceedings of the National Academy of Sciences 104, 98709875 (2007).

Vaidya, V. A., Marek, G. J., Aghajanian, G. K. \& Duman, R. S. 5-HT2A receptor-mediated regulation of brain-derived neurotrophic factor mRNA in the hippocampus and the neocortex. The Journal of neuroscience : the official journal of the Society for Neuroscience 17, 2785-2795 (1997).

Cavus, I. \& Duman, R. S. Influence of estradiol, stress, and 5-HT2A agonist treatment on brain-derived neurotrophic factor expression in female rats. Biological psychiatry 54, 59-69 (2003).

Martinowich, K., Manji, H. \& Lu, B. New insights into BDNF function in depression and anxiety. Nature neuroscience 10, 1089 (2007).

Carhart-Harris, R. L. et al. Neural correlates of the psychedelic state as determined by fMRI studies with psilocybin. Proceedings of the National Academy of Sciences 109, 2138-2143, doi:10.1073/pnas.1119598109 (2012).

Smigielski, L., Scheidegger, M., Kometer, M. \& Vollenweider, F. X. Psilocybin-assisted mindfulness training modulates selfconsciousness and brain default mode network connectivity with lasting effects. Neurolmage 196, 207-215 (2019).

Müller, F., Dolder, P. C., Schmidt, A., Liechti, M. E. \& Borgwardt, S. Altered network hub connectivity after acute LSD administration. Neurolmage: Clinical 18, 694-701, doi:https://doi.org/10.1016/j.nicl.2018.03.005 (2018).

Carhart-Harris, R. L. et al. Neural correlates of the LSD experience revealed by multimodal neuroimaging. Proceedings of the National Academy of Sciences of the United States of America 113, 4853-4858, doi:http://dx.doi.org/10.1073/pnas.1518377113 (2016).

Palhano-Fontes, F. et al. The psychedelic state induced by ayahuasca modulates the activity and connectivity of the default mode network. PLOS ONE 10, e0118143 (2015).

Roseman, L., Leech, R., Feilding, A., Nutt, D. J. \& Carhart-Harris, R. L. The effects of psilocybin and MDMA on betweennetwork resting state functional connectivity in healthy volunteers. Frontiers in Human Neuroscience 8 , doi:10.3389/fnhum.2014.00204 (2014).

Lord, L.-D. et al. Dynamical exploration of the repertoire of brain networks at rest is modulated by psilocybin. Neurolmage 199, 127-142, doi:https://doi.org/10.1016/j.neuroimage.2019.05.060 (2019).

Andrews-Hanna, J. R. The brain's default network and its adaptive role in internal mentation. Neuroscientist 18, 251-270, doi:10.1177/1073858411403316 (2012).

Raichle, M. E. et al. A default mode of brain function. Proceedings of the National Academy of Sciences 98, 676-682, doi:10.1073/pnas.98.2.676 (2001).

Davey, C. G., Pujol, J. \& Harrison, B. J. Mapping the self in the brain's default mode network. Neurolmage 132, 390-397, doi:10.1016/j.neuroimage.2016.02.022 (2016).

Atasoy, S. et al. Connectome-harmonic decomposition of human brain activity reveals dynamical repertoire reorganization under LSD. Scientific Reports 7, 17661, doi:10.1038/s41598-017-17546-0 (2017).

Tagliazucchi, E. et al. Increased Global Functional Connectivity Correlates with LSD-Induced Ego Dissolution. Current biology : CB 26, 1043-1050, doi:10.1016/j.cub.2016.02.010 (2016).

Carhart-Harris, R. L. et al. Functional connectivity measures after psilocybin inform a novel hypothesis of early psychosis. Schizophrenia bulletin 39, 1343-1351, doi:http://dx.doi.org/10.1093/schbul/sbs117 (2013).

Andrade, R. Serotonergic regulation of neuronal excitability in the prefrontal cortex. Neuropharmacology 61, 382-386, doi:10.1016/j.neuropharm.2011.01.015 (2011).

Bombardi, C. \& Di Giovanni, G. Functional anatomy of 5-HT2A receptors in the amygdala and hippocampal complex: relevance to memory functions. Experimental brain research 230, 427-439, doi:10.1007/s00221-013-3512-6 (2013).

Puig, M. V. \& Gulledge, A. T. Serotonin and prefrontal cortex function: neurons, networks, and circuits. Mol Neurobiol 44, 449-464, doi:10.1007/s12035-011-8214-0 (2011).

Vollenweider, F. et al. Positron emission tomography and fluorodeoxyglucose studies of metabolic hyperfrontality and psychopathology in the psilocybin model of psychosis. Neuropsychopharmacology : official publication of the American College of Neuropsychopharmacology 16, 357-372 (1997).

Carhart-Harris, R. L. et al. The entropic brain: a theory of conscious states informed by neuroimaging research with psychedelic drugs. Front Hum Neurosci 8 (2014).

Carhart-Harris, R. L. \& Friston, K. J. The default-mode, ego-functions and free-energy: a neurobiological account of Freudian ideas. Brain : a journal of neurology 133, 1265-1283, doi:10.1093/brain/awq010 (2010).

Lebedev, A. V. et al. Finding the self by losing the self: Neural correlates of ego-dissolution under psilocybin. Human brain mapping 36, 3137-3153 (2015).

Dittrich, A. The standardized psychometric assessment of altered states of consciousness (ASCs) in humans. Pharmacopsychiatry 31, 80-84 (1998).

Frahm, J. et al. Localized high-resolution proton NMR spectroscopy using stimulated echoes: initial applications to human brain in vivo. Magnetic resonance in medicine 9, 79-93 (1989). 
Near, J. et al. Preprocessing, analysis and quantification in single-voxel magnetic resonance spectroscopy: experts' consensus recommendations. NMR in biomedicine, e4257, doi:10.1002/nbm.4257 (2020). Whitfield-Gabrieli, S. \& Nieto-Castanon, A. Conn: a functional connectivity toolbox for correlated and anticorrelated brain networks. Brain connectivity 2, 125-141, doi:10.1089/brain.2012.0073 (2012). National Academy of Sciences of the United States of America 106, 13040-13045, doi:10.1073/pnas.0905267106 (2009). Sherry, A. \& Henson, R. K. Conducting and interpreting canonical cor
friendly primer. Journal of personality assessment 84, 37-48 (2005). Brown, R. T. et al. Pharmacokinetics of Escalating Doses of Oral Psilocybin in Healthy Adults. Clinical pharmacokinetics, doi:10.1007/s40262-017-0540-6 (2017). Abou-Elseoud, A. et al. The effect of model order selection in group PICA. Human brain mapping 31, 1207-1216, doi:10.1002/hbm.20929 (2010).

Allen, E. A. et al. A baseline for the multivariate comparison of resting-state networks. Frontiers in systems neuroscience 5, 2-2, doi:10.3389/fnsys.2011.00002 (2011). Disorder: Results from the EU-AIMS Longitudinal European Autism Project. Biological Psychiatry: Cognitive Neuroscience and Neuroimaging 4, 260-270, doi:https://doi.org/10.1016/j.bpsc.2018.11.010 (2019).

Weber, E. T. \& Andrade, R. Htr2a Gene and 5-HT(2A) Receptor Expression in the Cerebral Cortex Studied Using Genetically Modified Mice. Frontiers in neuroscience 4, doi:10.3389/fnins.2010.00036 (2010).

Marek, G. J. \& Aghajanian, G. K. The electrophysiology of prefrontal serotonin systems: therapeutic implications for mood and psychosis. Biological psychiatry 44, 1118-1127 (1998).

Lambe, E. K., Goldman-Rakic, P. S. \& Aghajanian, G. K. Serotonin Induces EPSCs Preferentially in Layer V Pyramidal Neurons of the Frontal Cortex in the Rat. Cerebral Cortex 10, 974-980, doi:10.1093/cercor/10.10.974 (2000).

Marek, G. J. \& Aghajanian, G. K. 5-Hydroxytryptamine-induced excitatory postsynaptic currents in neocortical layer $\mathrm{V}$ pyramidal cells: suppression by mu-opiate receptor activation. Neuroscience 86, 485-497, doi:10.1016/s03064522(98)00043-8 (1998).

Gouzoulis-Mayfrank, E. et al. Neurometabolic effects of psilocybin, 3, 4-methylenedioxyethylamphetamine (MDE) and dmethamphetamine in healthy volunteers: a double-blind, placebo-controlled PET study with [18F] FDG. Neuropsychopharmacology : official publication of the American College of Neuropsychopharmacology 20, 565-581 (1999).

Hermle, L. et al. Mescaline-induced psychopathological, neuropsychological, and neurometabolic effects in normal subjects: experimental psychosis as a tool for psychiatric research. Biological psychiatry 32, 976-991 (1992).

Riba, J. et al. Increased frontal and paralimbic activation following ayahuasca, the pan-Amazonian inebriant. Psychopharmacology (Berl) 186, 93-98, doi:10.1007/s00213-006-0358-7 (2006).

Zhou, F.-M. \& Hablitz, J. J. Activation of serotonin receptors modulates synaptic transmission in rat cerebral cortex. Journal of neurophysiology 82, 2989-2999 (1999).

Lewis, C. R. et al. Two dose investigation of the 5-HT-agonist psilocybin on relative and global cerebral blood flow. Neuroimage 159, 70-78 (2017).

McKenna, D. J., Repke, D. B., Lo, L. \& Peroutka, S. J. Differential interactions of indolealkylamines with 5hydroxytryptamine receptor subtypes. Neuropharmacology 29, 193-198, doi:10.1016/0028-3908(90)90001-8 (1990).

Blair, J. B. et al. Effect of ring fluorination on the pharmacology of hallucinogenic tryptamines. Journal of medicinal chemistry 43, 4701-4710 (2000).

Carhart-Harris, R. \& Nutt, D. Serotonin and brain function: a tale of two receptors. Journal of Psychopharmacology 31, 1091-1120 (2017).

Hamon, M. et al. The central 5-HT1A receptors: pharmacological, biochemical, functional, and regulatory properties. Annals of the New York Academy of Sciences 600, 114-129; discussion 129-131, doi:10.1111/j.1749-6632.1990.tb16877.x (1990).

Puts, N. A. J. \& Edden, R. A. E. In vivo magnetic resonance spectroscopy of GABA: a methodological review. Progress in nuclear magnetic resonance spectroscopy 60, 29-41, doi:10.1016/j.pnmrs.2011.06.001 (2012).

Shungu, D. C. et al. Brain gamma-aminobutyric acid (GABA) detection in vivo with the J-editing (1) H MRS technique: a comprehensive methodological evaluation of sensitivity enhancement, macromolecule contamination and test-retest reliability. NMR in biomedicine 29, 932-942, doi:10.1002/nbm.3539 (2016).

Schmitz, T. W., Correia, M. M., Ferreira, C. S., Prescot, A. P. \& Anderson, M. C. Hippocampal GABA enables inhibitory control over unwanted thoughts. Nature communications 8, 1311 (2017).

Vollenweider, F. et al. Metabolic hyperfrontality and psychopathology in the ketamine model of psychosis using positron emission tomography (PET) and [18F] fluorodeoxyglucose (FDG). European neuropsychopharmacology 7, 9-24 (1997). 


\section{Chapter 4}

Carhart-Harris, R. L. et al. The paradoxical psychological effects of lysergic acid diethylamide (LSD). Psychological medicine 46, 1379-1390 (2016).

Cortese, B. M. \& Phan, K. L. The role of glutamate in anxiety and related disorders. CNS spectrums 10, 820-830 (2005).

Grachev, I. D. \& Apkarian, A. V. Chemical mapping of anxiety in the brain of healthy humans: an in vivo $1 \mathrm{H}-\mathrm{MRS}$ study on the effects of sex, age, and brain region. Human brain mapping 11, 261-272, doi:10.1002/10970193(200012)11:4<261::aid-hbm30>3.0.co;2-6 (2000).

Mathew, S. J. et al. Open-label trial of riluzole in generalized anxiety disorder. The American journal of psychiatry 162 , 2379-2381, doi:10.1176/appi.ajp.162.12.2379 (2005).

Buckholtz, N. S., Zhou, D. F., Freedman, D. X. \& Potter, W. Z. Lysergic acid diethylamide (LSD) administration selectively downregulates serotonin2 receptors in rat brain. Neuropsychopharmacology : official publication of the American College of Neuropsychopharmacology 3, 137-148 (1990).

Acioly, M. A., Carvalho, C. H., Tatagiba, M. \& Gharabaghi, A. The parahippocampal gyrus as a multimodal association area in psychosis. Journal of Clinical Neuroscience 17, 1603-1605 (2010).

Friston, K., Liddle, P., Frith, C., Hirsch, S. \& Frackowiak, R. The left medial temporal region and schizophrenia: a PET study. Brain 115, 367-382 (1992).

Lambert, M. V., Sierra, M., Phillips, M. L. \& David, A. S. The spectrum of organic depersonalization: a review plus four new cases. The Journal of neuropsychiatry and clinical neurosciences 14, 141-154 (2002).

Lemche, E. et al. Dissociable brain correlates for depression, anxiety, dissociation, and somatization in depersonalizationderealization disorder. CNS spectrums 21, 35-42 (2016).

Millière, R., Carhart-Harris, R. L., Roseman, L., Trautwein, F.-M. \& Berkovich-Ohana, A. Psychedelics, Meditation, and SelfConsciousness. Frontiers in psychology 9, 1475-1475, doi:10.3389/fpsyg.2018.01475 (2018).

Oehen, P., Traber, R., Widmer, V. \& Schnyder, U. A randomized, controlled pilot study of MDMA (+/- 3,4Methylenedioxymethamphetamine)-assisted psychotherapy for treatment of resistant, chronic Post-Traumatic Stress Disorder (PTSD). Journal of psychopharmacology (Oxford, England) 27, 40-52, doi:10.1177/0269881112464827 (2013).

Garcia-Romeu, A., Griffiths, R. R. \& Johnson, M. W. Psilocybin-occasioned Mystical Experiences in the Treatment of Tobacco Addiction. Current drug abuse reviews 7, 157-164 (2015).

Roseman, L., Nutt, D. J. \& Carhart-Harris, R. L. Quality of Acute Psychedelic Experience Predicts Therapeutic Efficacy of Psilocybin for Treatment-Resistant Depression. Frontiers in Pharmacology 8, 974, doi:10.3389/fphar.2017.00974 (2017). Uthaug, M. et al. A single inhalation of vapor from dried toad secretion containing 5-methoxy-N, N-dimethyltryptamine (5-MeO-DMT) in a naturalistic setting is related to sustained enhancement of satisfaction with life, mindfulness-related capacities, and a decrement of psychopathological symptoms. Psychopharmacology, 1-14 (2019).

Ly, C. et al. Psychedelics Promote Structural and Functional Neural Plasticity. Cell reports 23, 3170-3182, doi:10.1016/j.celrep.2018.05.022 (2018).

Gewirtz, J. C., Chen, A. C., Terwilliger, R., Duman, R. C. \& Marek, G. J. Modulation of DOI-induced increases in cortical BDNF expression by group II mGlu receptors. Pharmacology, biochemistry, and behavior 73, 317-326, doi:10.1016/s00913057(02)00844-4 (2002).

Jones, K. A. et al. Rapid modulation of spine morphology by the 5-HT2A serotonin receptor through kalirin-7 signaling. Proceedings of the National Academy of Sciences 106, 19575-19580 (2009).

Manji, H. K., Moore, G. J., Rajkowska, G. \& Chen, G. Neuroplasticity and cellular resilience in mood disorders. Molecular psychiatry 5, 578-593, doi:10.1038/sj.mp.4000811 (2000).

Studerus, E., Kometer, M., Hasler, F. \& Vollenweider, F. X. Acute, subacute and long-term subjective effects of psilocybin in healthy humans: a pooled analysis of experimental studies. Journal of psychopharmacology (Oxford, England) 25, 14341452, doi:10.1177/0269881110382466 (2011).

Jezzard, P. Correction of geometric distortion in fMRI data. Neurolmage 62, 648-651, doi:https://doi.org/10.1016/j.neuroimage.2011.09.010 (2012).

Birn, R. M. et al. The effect of scan length on the reliability of resting-state fMRI connectivity estimates. Neurolmage 83, 550-558, doi:10.1016/j.neuroimage.2013.05.099 (2013).

Allen, M., Poggiali, D., Whitaker, K., Marshall, T. R. \& Kievit, R. A. Raincloud plots: a multi-platform tool for robust data visualization. Wellcome Open Res 4, 63-63, doi:10.12688/wellcomeopenres.15191.1 (2019). 


\section{Supplemental Information}

\section{Material and Methods}

Participants. Participants were recruited through advertisements around Maastricht University and internet forum in the Netherlands. Inclusion criteria were: age, 18-40 years; previous experience with a psychedelic drug, but not within the past 3 months; normal weight, body mass index between 18 and $28 \mathrm{~kg} / \mathrm{m} 2$; free from psychotropic medication; good physical health, including absence of major medical, endocrine, and neurological conditions; and written informed consent. Exclusion criteria were: history of drug abuse or addiction, which were determined by medical questionnaires and examination; pregnancy or lactation; health issues including hypertension (diastolic >90 and systolic >140), cardiac dysfunction, and liver dysfunction; current or history of psychiatric disorders; previous experience of serious side effects to cannabis; and MRI contraindications. Before inclusion, subjects were screened and examined by a study physician, who checked for general health, conducted a resting ECG, and took blood and urine samples in which hematology, clinical chemistry, urine, and virology analyses were conducted. Participant demographic data can be found in Table S1.

Psilocybin (powder) was obtained from GH Pharm GmbH, Frankfurt, Germany. A permit for obtaining, storing, and administering psilocybin was obtained from the Dutch Drug Enforcement Administration. Participants were financially compensated for their participation in the study.

Procedure. Participants were familiarized with the test day procedures on a separate training day prior to the treatment conditions. Participants were instructed to refrain from drug use, including psychedelic drugs ( $\geq 3$ months), MDMA/ecstasy ( $\geq 14$ days), alcohol ( $\geq 24$ hours), and all other drugs of abuse ( $\geq 7$ days) prior to their testing day. Additionally, participants were asked to refrain from caffeine and nicotine use the day of the test day.

On arrival of a test day, absence of drug and alcohol use was assessed via a urine drug screen and a breath alcohol screen. An additional pregnancy test was given if participants were female. If all tests were found to be negative, participants were allowed to proceed, and a venal catheter was placed, in order to take blood samples throughout the testing day. Before administration of treatment, a baseline blood sample was taken and baseline vital signs (blood pressure and heart rate) were measured. After measurements, the treatment was administered orally, in a closed cup containing bitter lemon (placebo) or bitter lemon and psilocybin (powder). After 40 minutes, participants were placed in the MRI scanner, where resting state scans and magnetic resonance spectroscopy were performed throughout a 1 hour time window. At the end of the test day (approximately 6 hours after treatment administration), participants were 
asked to complete measures of retrospective subjective high. Participants stayed under supervision until the testing day was complete, and the researcher deemed they were fit to go home.

\section{Questionnaires}

5-Dimensional Altered States of Consciousness Rating Scale. The 5D-ASC is a 94-item self-report scale that assesses the participants' alterations from normal waking consciousness ${ }^{1}$. The participant is asked to make a vertical mark on the $10-\mathrm{cm}$ line below each statement to rate to what extent the statements applied to their experience in retrospect from "No, not more than usually" to "Yes, more than usually." The 5D-ASC contains the 5 key dimensions, including anxious ego dissolution, visual restructuralization, auditory alterations, reduction of vigilance, and oceanic boundlessness; which can be broken down into 11 subscales consisting of experience of unity, spiritual experience, blissful state, insightfulness, disembodiment, impaired control and cognition, anxiety, complex imagery, elementary imagery, audio-visual synesthesia, and changed meaning of percepts.

Ego Dissolution Inventory. The Ego Dissolution Inventory (EDI) is an eight-item self-report scale that assesses the participant's experience of ego dissolution ${ }^{2}$. Sample items for the scale includes the following: "I experienced a dissolution of my 'self' or ego" and "I felt at one with the universe." The participants answered the scale with endpoints of either $0=$ "No, not more than usually" or $100=$ "Yes I experience this completely/entirely." The EDI is scored by calculating the mean of all the 8 items (range 0-100). The higher the total score, the stronger the experience of ego dissolution.

Satisfaction with Life Scale. The Satisfaction with Life Scale (SWLS) is a 5-item questionnaire designed to measure global cognitive judgments of satisfaction with one's life ${ }^{3}$, and has been used to measure the life satisfaction component of subjective well-being ${ }^{3,4}$. Individuals answer each item on a Likert-scale ranging from 1 (strongly disagree) to 7 (strongly agree). The total score is then obtained by summing the ratings from each item. Sample items include "In most ways my life is close to my ideal" and "If I could live my life over, I would change almost nothing". The minimum possible score is 5, and a maximum possible score of 35, with a score of 5-19 defined as dissatisfied to below average life satisfaction, 20-24 defined as average life satisfaction, and 29-35 defined as high to very high life satisfaction ${ }^{5}$.

\section{Blood}

All samples were centrifuged and serum was frozen at $-20^{\circ} \mathrm{C}$, and kept in the dark until analysis. Analysis of psilocine in serum was performed according to Martin, et al. ${ }^{6}$. Serum (200 $\mu$ l) was extracted with $1 \mathrm{ml}$ of ethyl acetate after addition of phosphate buffer $\mathrm{pH}$ 9, 20 ng psilocine- $\mathrm{d}_{10}$ 
and $10 \mu \mathrm{l}$ of $0.1 \mathrm{M}$ ascorbic acid for stabilization. The organic phase was evaporated and reconstituted with $100 \mu \mathrm{l}$ of $0.1 \%$ formic acid/acetonitrile $(80: 20, v / v)$. The analysis of $2 \mu \mathrm{l}$ was performed on an Agilent (Waldbronn, Germany) LC-MS/MS system consisting of a 1290 Infinity Liquid Chromatograph coupled via JetStream Electrospray Interface (ESI) to a G6460A Triple Quadrupole Mass Spectrometer. Analytes were separated on a Kinetex ${ }^{\circledR} 2.6 \mu \mathrm{m}$ XB-C18 $100 \AA$ AC column $(100 \times 2.1 \mathrm{~mm}$ ) plus corresponding guard column from Phenomenex (Aschaffenburg, Germany) at $30{ }^{\circ} \mathrm{C}$. Gradient elution at a flow rate of $0.5 \mathrm{ml} / \mathrm{min}$ using $0.01 \%$ formic acid containing $5 \mathrm{mM}$ ammonium formate $(\mathrm{A})$ and acetonitrile containing $0.1 \%$ formic acid (B) started with $5 \% \mathrm{~B}$, increased to $95 \% \mathrm{~B}$ during $4 \mathrm{~min}$ and was held for 2 min. Source parameters were: gas temperature $300{ }^{\circ} \mathrm{C}$, gas flow $11 \mathrm{l} / \mathrm{min}$, nebulizer $45 \mathrm{psi}$, sheath gas temperature $400{ }^{\circ} \mathrm{C}$, sheath gas flow $12 \mathrm{l} / \mathrm{min}$ and capillary voltage $3500 \mathrm{~V}$. Detection was performed in the multiple reaction monitoring mode $\left(\mathrm{m} / \mathrm{z}\right.$, collision energy in parentheses, quantifier underlined): psilocine- $\mathrm{d}_{10}$ : $215^{\circledR} 66$ (12), psilocine ${\underline{205^{\circledR}}}^{\circledR 8}(12) ; 205^{\circledR} 160$ (16). Five calibration standards were prepared from human serum with psilocine reference substance (LGC Standards GmbH, Wesel, Germany) and analyzed with the samples. The calibration was linear (regression coefficient $>0.999$ ) in the range $1-100 \mathrm{ng} / \mathrm{ml}$ with limits of detection and quantification below $0.5 \mathrm{ng} / \mathrm{ml}$.

\section{MRS Acquisition.}

Anatomical ( $T_{1}$-weighted) images were acquired using magnetisation-prepared 2 rapid acquisition gradient-echo (MP2RAGE) ${ }^{7}$ sequence $\left(T R=4.5 \mathrm{~s}, \mathrm{TE}=2.39 \mathrm{~ms}, \mathrm{Tl}_{1}=0.90 \mathrm{~s}, \mathrm{Tl}_{2}=2.75 \mathrm{~s}\right.$, flip angle ${ }_{1}$ $=5^{\circ}$, flip angle ${ }_{2}=3^{\circ}$, voxel size $=0.9 \mathrm{~mm}$ isotropic, matrix size $=256 \times 256 \times 192$, phase partial Fourier $=6 / 8$, GRAPPA factor $=3$ with 24 reference lines, bandwidth $=250 \mathrm{~Hz} /$ pixel, acquisition time $=6: 00 \mathrm{~min})$. Tissue probability maps for grey matter $(\mathrm{GM})$, white matter (WM) and cerebrospinal fluid (CSF) were generated from the $\mathrm{T}_{1}$-weighted anatomical images using FSL-FAST 8 , and assessed for differences in spectroscopic voxels, between groups.

Single-voxel proton magnetic resonance spectroscopy (MRS) measurements were performed on a MAGNETOM 7T MR scanner (Siemens Healthineers, Erlangen, Germany) with a whole-body gradient set (SC72; maximum amplitude, $70 \mathrm{mT} / \mathrm{m}$; maximum slew rate, $200 \mathrm{~T} / \mathrm{m} / \mathrm{s}$ ) and using an single-channel transmit/32-channel receive head coil (Nova Medical, Wilmington, MA, USA). Spectroscopic voxels of interest were placed by a trained operator at the medial prefrontal cortex (voxel size $=25 \mathrm{~mm} \times 20 \mathrm{~mm} \times 17 \mathrm{~mm}$ ) and the right hippocampus (voxel size = $37 \mathrm{~mm} \times 15 \mathrm{~mm} \times 15 \mathrm{~mm}$ ). Spectra were acquired with stimulated echo acquisition mode (STEAM)

9 sequence using the following parameters: $\mathrm{TE}=6.0 \mathrm{~ms}, \mathrm{TM}=10.0 \mathrm{~ms}$, TR $=5.0 \mathrm{~s}, \mathrm{NA}=64$, flip angle $=90^{\circ}, \mathrm{RF}$ bandwidth $=4.69 \mathrm{kHz}$, RF centred at $2.4 \mathrm{ppm}$, receive bandwidth $=4.0 \mathrm{kHz}$, vector size $=2048,16$-step phase cycling, acquisition time $=5: 20$ min. Water suppression was achieved by variable power RF pulses with optimised relaxation delays (VAPOR) ${ }^{10}$. In addition, a complete 
phase cycle of measurements was acquired without the water suppression RF pulses to record a water peak reference for eddy current correction ${ }^{11}$ and absolute metabolite concentration calibration ${ }^{12,13}$. Before the spectroscopy measurements, a 3D-GRE dual-echo field-map $\left(\mathrm{TE}_{1}=1.00\right.$ $\mathrm{ms}, \mathrm{TE}_{2}=2.98 \mathrm{~ms}, \mathrm{TR}=20.0 \mathrm{~ms}$, flip angle $=8^{\circ}$, voxel size $=3 \mathrm{~mm}$ isotropic, matrix size $=84 \times 84$ $\times 56$, bandwidth $=1450 \mathrm{~Hz} /$ pixel, acquisition time $=2: 24 \mathrm{~min}$ ) was acquired and used to calculate the shim currents required to homogenise the static magnetic field in the spectroscopic voxels of interest.

The spectra were analysed with LCModel version 6.3-1H using a GAMMA ${ }^{14}$ simulated basis set which includes Alanine (Ala), Ascorbic Acid (Asc), Aspartate (Asp), Creatine (Cr), y-Aminobutyric Acid (GABA), Glucose (Glc), Glutamate (Glu), Glutamine (Gln), Glycerophosphocholine (GPC), Glutathione (GSH), Glycine (Glyc), Lactate (Lac), Myo-Inositol (ml), N-Acetyl Aspartate (NAA), NAcetyl Aspartyl Glutamate (NAAG), Phosphocreatine (PCr), Phosphorylcholine (PCh), Phosphorylethanolamine (PE), Scyllo-Inositol (Scyllo), and Taurine (Tau) ${ }^{15}$. The metabolite basis set also includes an in vivo Macromolecules (MMol) spectrum which was collected using a metabolites suppressed double inversion recovery (DIR) STEAM with the same parameters as above and $\mathrm{TI}_{1}=2.09 \mathrm{~s}$ and $\mathrm{TI}_{2}=0.52 \mathrm{~s}^{16}$. All metabolite concentrations were reported with respect to total creatine. As metabolites were reported as a ratio to $t C R$, absolute metabolite concentration of tCR was checked in each brain region, for significant difference between groups. There was no significant difference between groups of tCR in either the mPFC or the hippocampus suggesting that any reported significant relative metabolite concentrations were not due to an increase or decrease in tCR

MRS Quality. To ensure data quality and reliable metabolite estimation, only absolute metabolite values with a relative Cramer-Rao lower bound below 20\%, a signal-to-noise ratio (SNR) greater than 10 , and a full-width at half-maximum peak height $(\mathrm{FWHM})<0.1$ were considered $^{17-19}$. MRS voxel placement and mean SNR, \%CRLB, and FWHM values can be found in Table 1. An overview of data points that were not included in the analysis, and reason why, can be found in Table S6.

\section{Preprocessing.}

Data was processed and analysed using the CONN toolbox 18.b20 (http://www.nitrc.org/projects/conn) based on SPM12 (http://www.fil.ion.ucl.ac.uk/spm/) running in MATLAB 2019a. Before quality assessement, FMRI data was available for 26 subjects in the psilocybin group and 27 subjects in the placebo group.

All functional volumes were realigned, unwarped, segmented into grey and white matter and cerebrospinal fluid, normalised into a standard stereotactic space (Montreal Neurological Institute; $\mathrm{MNI}$ ) and smoothed with a $6 \mathrm{~mm}$ full width at half maximum Gaussian kernel. The first 
two volumes were excluded in order to to ensure magnetization equilibrium. No slice-time correction was performed as data was acquired using multi-band acquisition.

All individual $\mathrm{T}_{1}$-weighted structural volumes were segmented into grey and white matter and cerebrospinal fluid and normalised to MNI space. Noise correction of the functional images included scrubbing with a global signal threshold of $z>3$ and a composite subject motion threshold of $>0.5 \mathrm{~mm}$ using ART as implemented in CONN, linear detrending, linear regression of the six motion parameters, and the white matter and cerebrospinal fluid signals, using the individual tissue masks obtained from the $T_{1}$-weighted structural images. Five principal components were extracted from white matter and cerebrospinal fluid signals (using individual tissue masks obtained from the T1-weighted structural images) and removed using CompCor ${ }^{21}$. These components are thought to reflect noise (especially motion and physiological fluctuations) and are therefore removed from the time series. The resulting functional images were band-pass filtered $(0.008<f$ $<0.09 \mathrm{~Hz}$ ) as it was found that band-pass filtering improves independent component results in addition to high-pass filtering (Pignat et al., 2013).

Quality assessment comprised three stages: Firstly, all scans were assessed with regard to the percentage of the scrubbed volumes. Subjects were only included in further analysis, if $\geq 5 \mathrm{~min}$ of the scan remained after scrubbing (corresponding to $<83.6 \%$ of the initial volumes). This was based on literature indicating that resting state scans $<5$ min are not reliable (Birn et al., 2013). Secondly, head motion after scrubbing was assessed using maximum framewise displacement (FD; sphere radius $50 \mathrm{~mm}$ ) calculated according to Power et al. (Power et al., 2012). Subjects were excluded if maximum FD was $>0.75 \mathrm{~mm}$ (half-voxel size). Four subjects (psilocybin group: 3; placebo group: 1) were excluded based on the first criterium and one subject (psilocybin group) was excluded based on the second criterium. The final sample thus consisted of 22 subjects in the psilocybin group and 26 subjects in the placebo group. All further analyses were based on this sample. Furthermore, we tested for significant differences between the groups of this sample. Mean FD before scrubbing was $0.18 \mathrm{~mm}( \pm$ SD 0.05) in the psilocybin group and $0.18 \mathrm{~mm}( \pm$ SD $0.06)$ in the placebo group. Average maximum FD before scrubbing in the psilocybin group was $0.98 \mathrm{~mm}( \pm$ SD 0.78) in the psilocybin group and $0.99 \mathrm{~mm}( \pm$ SD 0.95) in the placebo group. On average 10.90 volumes per scan ( \pm SD 9.49) were scrubbed in the psilocybin group and 10.50 volumes per scan ( \pm SD 9.80) in the placebo group. Mean FD after scrubbing was $0.17 \mathrm{~mm}( \pm$ SD $0.05)$ in the psilocybin group and $0.17 \mathrm{~mm}( \pm$ SD 0.05) in the placebo group. Average maximum FD before scrubbing in the psilocybin group was $0.51 \mathrm{~mm}( \pm$ SD 0.12 ) and $0.50 \mathrm{~mm}( \pm$ SD 0.13) in the placebo group. For comparison between groups, Mann-Whitney $U$ tests were performed because the assumption for normality distribution was not met in several cases (assessed with ShapiroWilk tests). There were no significant differences between groups for any of these measures 
(invalid volumes: $p=0.77$; mean FD before scrubbing: $p=0.72$; average maximum FD before scrubbing: $p=0.81$; mean FD after scrubbing: $p=0.81$; average maximum FD after scrubbing: $p=0.70)$.

\section{Independent component analysis.}

Independent component analysis (ICA) was performed using group-ICA procedures implemented in the CONN toolbox following methods described by Calhoun et at. (Calhoun et al., 2001). ICA results are determined by the chosen number of dimensions, i.e. a higher number of dimensions might result in a higher number of distinct resting state networks compared with a lower number of dimensions. Dimensionality reduction on the subject-level was set to 64. Independent components were restricted to 20 in order to allow comparisons with 10 established resting state network described by Smith et al. (Smith et al., 2009) and previous studies on psilocybin (CarhartHarris et al., 2013) and LSD (Carhart-Harris et al., 2016; Müller et al., 2018), which also applied comparable restrictions. Decisions regarding the labelling of the networks identified in this data set were based on visual inspection (Kelly Jr. et al., 2010) and cross-correlation of the unthresholded ICA components with the unthresholded resting state networks described by Smith et al. (https://www.fmrib.ox.ac.uk/datasets/brainmap+rsns/). 
Psilocybin-induced changes in glutamate, network connectivity, and ego dissolution

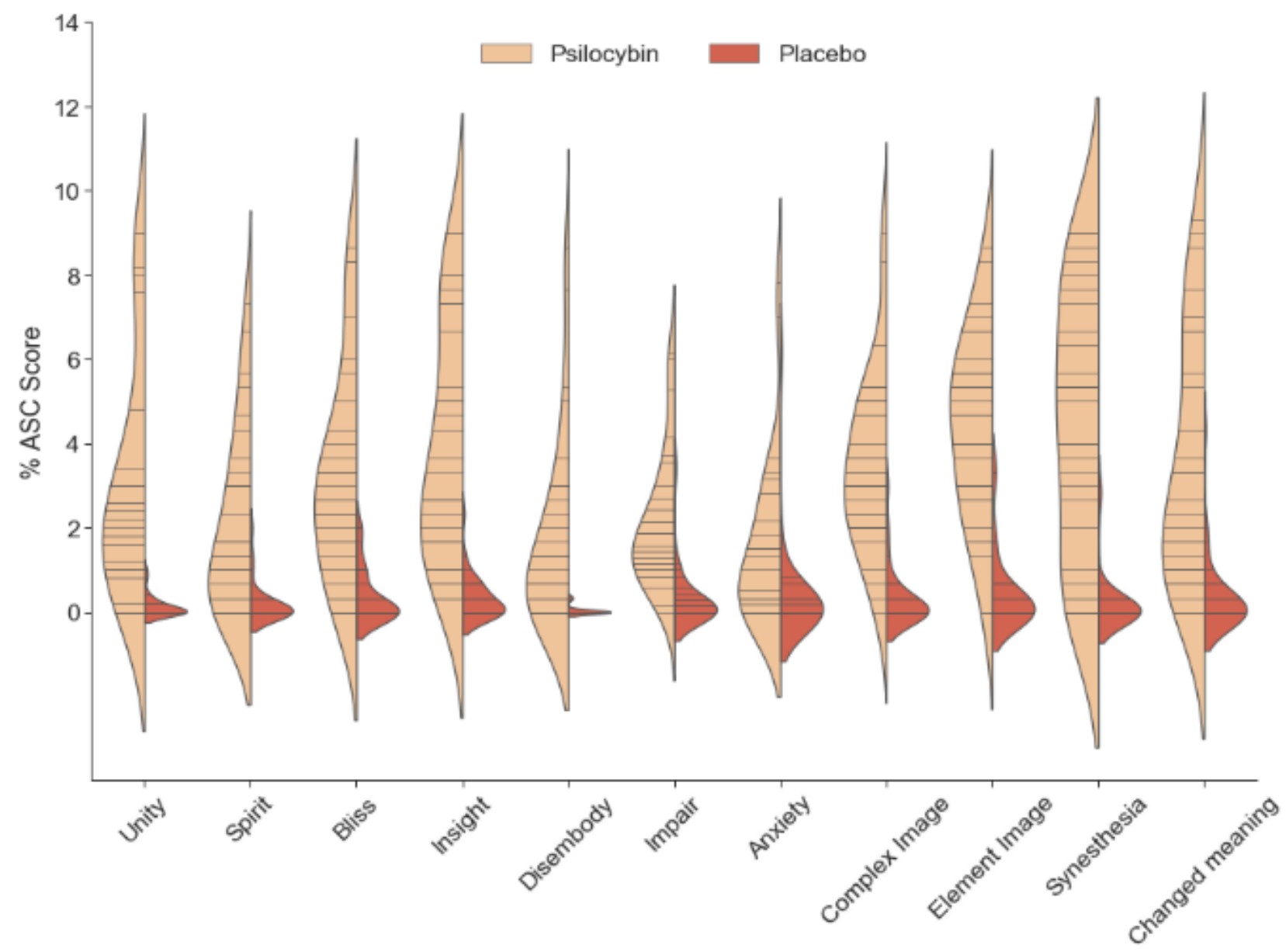

Figure S1. Violin plots displaying reported scores on the 11 sub-dimensions of the 5D-ASC. 
A.

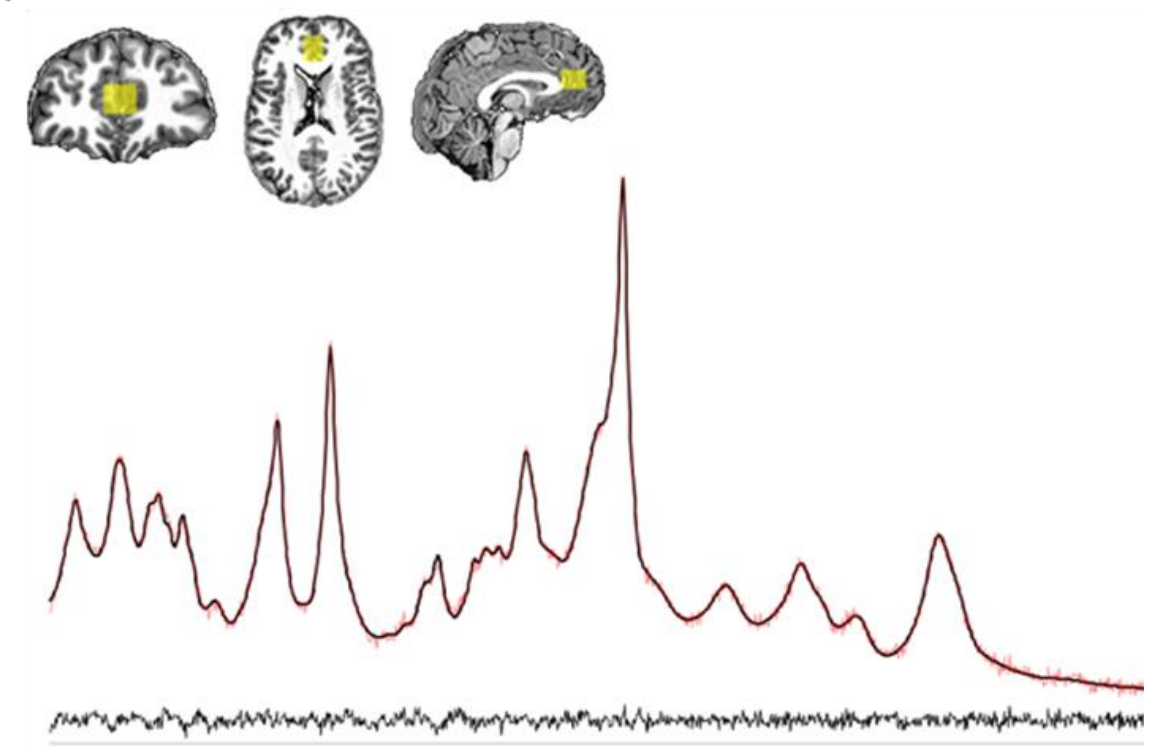

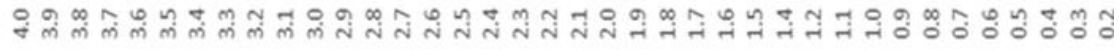

Chemical Shift (ppm)

B.

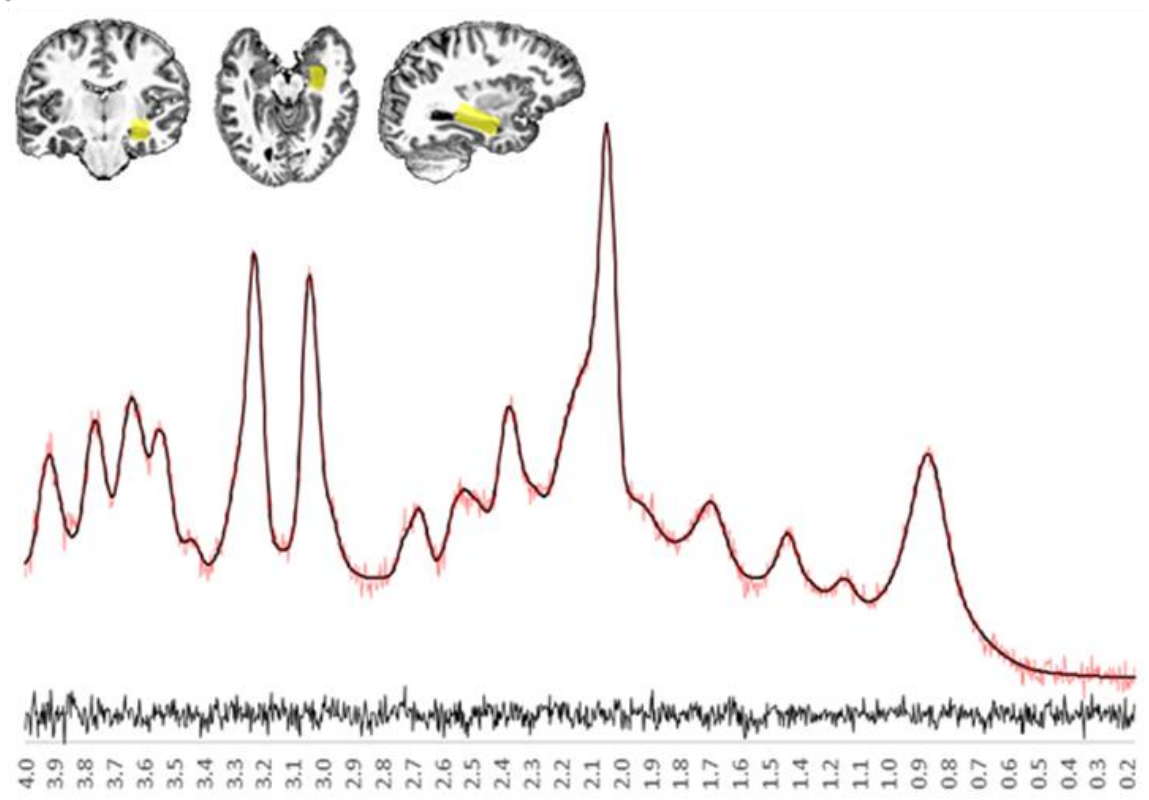

Chemical Shift (ppm)

Figure S2. Example LC-Model fitted 1H-MRS data for the medial prefrontal cortex (A) and hippocampus (B). The black line spectra corresponds to the phased $1 \mathrm{H}-\mathrm{MRS}$ data with the LC-Model fits overlaid (red). The residual spectra (raw data minus the LC-Model fit) are displayed below the spectrum. 
Psilocybin-induced changes in glutamate, network connectivity, and ego dissolution

Table S1. Mean subject characteristics (SD) and history of drug use for healthy participants in the psilocybin and the placebo condition ( $\mathrm{N}=60)$.

\begin{tabular}{|c|c|c|c|c|}
\hline Variable & Psilocybin & Placebo & Value & $P$ value \\
\hline Gender (male/female), n, total & $18 / 12,30$ & $17 / 13,30$ & $\chi 2=0.07^{\ddagger}$ & 0.79 \\
\hline Age, years & $22.73(2.90)$ & $23.20(3.65)$ & $t=-0.55^{+}$ & 0.60 \\
\hline History of psychedelic use, years & $2.92(2.62)$ & $2.19(2.55)$ & $t=1.04$ & 0.30 \\
\hline $\begin{array}{l}\text { Lifetime psychedelic use, number of } \\
\text { occasions }\end{array}$ & $9.53(16.81)$ & $5.47(8.24)$ & $t=1.09$ & 0.28 \\
\hline Cannabis consumption, per month & $2.67(3.14)$ & $3.24(4.91)$ & $t=-0.47^{+}$ & 0.64 \\
\hline $\begin{array}{l}\text { Alcohol consumption, glasses per } \\
\text { week }\end{array}$ & $5.47(4.78)$ & $5.82(4.02)$ & $t=0.31^{+}$ & 0.76 \\
\hline $\begin{array}{l}\text { Caffeine consumption, glasses per } \\
\text { week }\end{array}$ & $10.00(7.75)$ & $9.38(8.10)$ & $t=0.30^{+}$ & 0.76 \\
\hline $\begin{array}{l}\text { Nicotine consumption, cigarettes } \\
\text { per week }\end{array}$ & $3.82(15.66)$ & $7.67(15.49)$ & $t=-0.96^{+}$ & 0.34 \\
\hline
\end{tabular}

\footnotetext{
*Significant $P$ values

${ }^{\dagger}$ Independent $t$ test

${ }^{\ddagger} \chi 2$ test for frequency data
} 


\section{Chapter 4}

Table S2. Time course for mean (S.E.) concentrations of psilocin in serum (ng/ml), as determined by liquid chromatography-mass spectrometry (LC-MSMS).

\begin{tabular}{ll}
\hline $\begin{array}{l}\text { Time relative to treatment } \\
\text { intake (minutes) }\end{array}$ & Psilocin $(\mathrm{ng} / \mathrm{ml})$ \\
80 & $15.61(1.66)$ \\
150 & $12.86(1.13)$ \\
360 & $4.85(0.54)$ \\
\hline
\end{tabular}


Psilocybin-induced changes in glutamate, network connectivity, and ego dissolution

Table S3. Mean (S.E.) metabolite concentrations for each treatment condition, including metabolites that were quantified in the spectra, but not of focus for this study. Also included are grey matter, white matter, and CSF amounts in each voxel. ${ }^{*} p<.05$

\begin{tabular}{|c|c|c|c|c|c|c|c|c|c|c|c|c|c|c|}
\hline \multirow[t]{2}{*}{ Focus of study } & \multicolumn{3}{|l|}{$\mathrm{Glu} / \mathrm{tCr}$} & \multicolumn{3}{|c|}{$\mathrm{GABA} / \mathrm{tCr}$} & \multicolumn{3}{|c|}{$\begin{array}{l}\mathrm{NAA}+ \\
\mathrm{NAAG} / \mathrm{tCr}\end{array}$} & \multicolumn{3}{|l|}{$\mathrm{ml} / \mathrm{tCr}$} & \multicolumn{2}{|l|}{$\mathrm{tCr}$} \\
\hline & Psi & Pla & $p$ & Psi & Pla & $p$ & Psi & Pla & $p$ & Psi & Pla & $p$ & Psi & Pla \\
\hline $\begin{array}{l}\text { Medial } \\
\text { prefrontal } \\
\text { cortex }\end{array}$ & $\begin{array}{l}1.23 \\
(0.02)\end{array}$ & $\begin{array}{l}1.14 \\
(0.02)\end{array}$ & $.01^{*}$ & $\begin{array}{l}0.17 \\
(0.01)\end{array}$ & $\begin{array}{l}0.14 \\
(0.01)\end{array}$ & $.01^{*}$ & $\begin{array}{l}1.41 \\
(0.03)\end{array}$ & $\begin{array}{l}1.31 \\
(0.02)\end{array}$ & $.02 *$ & $\begin{array}{l}0.78 \\
(0.03)\end{array}$ & $\begin{array}{l}0.78 \\
(0.02)\end{array}$ & .95 & $\begin{array}{l}3.69 \\
(0.10)\end{array}$ & $\begin{array}{l}4.10 .07 \\
(0.16)\end{array}$ \\
\hline Hippocampus & $\begin{array}{l}0.77 \\
(0.03)\end{array}$ & $\begin{array}{l}0.88 \\
(0.03)\end{array}$ & $.03 *$ & $\begin{array}{l}0.17 \\
(0.01)\end{array}$ & $\begin{array}{l}0.20 \\
(0.04)\end{array}$ & .88 & $\begin{array}{l}1.41 \\
(0.05)\end{array}$ & $\begin{array}{l}1.36 \\
(0.04)\end{array}$ & .40 & $\begin{array}{l}1.00 \\
(0.05)\end{array}$ & $\begin{array}{l}1.04 \\
(0.04)\end{array}$ & .39 & $\begin{array}{l}4.28 \\
(0.21)\end{array}$ & $\begin{array}{l}4.20 .43 \\
(0.19)\end{array}$ \\
\hline \multirow[t]{2}{*}{ Extra } & \multicolumn{3}{|l|}{$\mathrm{Gln} / \mathrm{tCr}$} & \multicolumn{3}{|l|}{$\mathrm{Glx} / \mathrm{tCr}$} & \multicolumn{3}{|l|}{$\mathrm{Cr} / \mathrm{tCr}$} & \multicolumn{3}{|l|}{$\mathrm{PCr} / \mathrm{tCr}$} & \multicolumn{2}{|l|}{$\mathrm{NAA} / \mathrm{tCr}$} \\
\hline & Psi & Pla & $p$ & Psi & Pla & $p$ & Psi & Pla & $p$ & Psi & Pla & $p$ & Ps & Pla \\
\hline $\begin{array}{l}\text { Medial } \\
\text { prefrontal } \\
\text { cortex }\end{array}$ & $\begin{array}{l}0.28 \\
(0.01)\end{array}$ & $\begin{array}{l}0.28 \\
(0.01)\end{array}$ & .71 & $\begin{array}{l}1.51 \\
(0.03)\end{array}$ & $\begin{array}{l}1.42 \\
(0.02)\end{array}$ & $.03 *$ & $\begin{array}{l}0.51 \\
(0.04)\end{array}$ & $\begin{array}{l}0.46 \\
(0.33)\end{array}$ & .47 & $\begin{array}{l}0.63 \\
(0.03)\end{array}$ & $\begin{array}{l}0.64 \\
(0.03)\end{array}$ & .78 & $\begin{array}{l}1.35 \\
(0.03)\end{array}$ & $\begin{array}{l}1.25 .03 * \\
(0.02)\end{array}$ \\
\hline Hippocampus & $\begin{array}{l}0.21 \\
(0.03)\end{array}$ & $\begin{array}{l}0.18 \\
(0.02)\end{array}$ & .56 & $\begin{array}{l}0.87 \\
(0.05)\end{array}$ & $\begin{array}{l}1.02 \\
(0.03)\end{array}$ & $.01^{*}$ & $\begin{array}{l}0.72 \\
(0.07)\end{array}$ & $\begin{array}{l}0.69 \\
(0.06)\end{array}$ & .68 & $\begin{array}{l}0.79 \\
(0.05)\end{array}$ & $\begin{array}{l}0.73 \\
(0.05)\end{array}$ & .36 & $\begin{array}{l}1.41 \\
(0.05)\end{array}$ & $\begin{array}{l}1.36 .40 \\
(0.04)\end{array}$ \\
\hline \multirow[t]{2}{*}{ Extra } & \multicolumn{3}{|c|}{ tCho/tCr } & \multicolumn{3}{|c|}{$\mathrm{GSH} / \mathrm{tCr}$} & \multicolumn{3}{|c|}{ Grey matter } & \multicolumn{2}{|c|}{ White matter } & & \multicolumn{2}{|l|}{ CSF } \\
\hline & Psi & Pla & $p$ & Psi & Pla & $p$ & Psi & Pla & $p$ & Psi & Pla & $p$ & Psi & Pla \\
\hline $\begin{array}{l}\text { Medial } \\
\text { prefrontal } \\
\text { cortex }\end{array}$ & $\begin{array}{l}0.16 \\
(0.01)\end{array}$ & $\begin{array}{l}0.15 \\
(0.01)\end{array}$ & .73 & $\begin{array}{l}0.19 \\
(0.01)\end{array}$ & $\begin{array}{l}0.19 \\
(0.01)\end{array}$ & .75 & $\begin{array}{l}0.53 \\
(0.01)\end{array}$ & $\begin{array}{l}0.53 \\
(0.01)\end{array}$ & .88 & $\begin{array}{l}0.15 \\
(0.01)\end{array}$ & $\begin{array}{l}0.14 \\
(0.004)\end{array}$ & .62 & $\begin{array}{l}0.32 \\
(0.01)\end{array}$ & $\begin{array}{l}0.32 .60 \\
(0.01)\end{array}$ \\
\hline Hippocampus & $\begin{array}{l}0.17 \\
(0.01)\end{array}$ & $\begin{array}{l}0.20 \\
(0.01)\end{array}$ & $.03 *$ & $\begin{array}{l}0.24 \\
(0.02)\end{array}$ & $\begin{array}{l}0.28 \\
(0.02)\end{array}$ & .41 & $\begin{array}{l}0.43 \\
(0.01)\end{array}$ & $\begin{array}{l}0.44 \\
(0.01)\end{array}$ & 0.62 & $\begin{array}{l}0.43 \\
(0.01)\end{array}$ & $\begin{array}{l}0.45 \\
(0.01)\end{array}$ & 0.23 & $\begin{array}{l}30.13 \\
(0.01)\end{array}$ & $\begin{array}{l}0.110 .08 \\
(0.01)\end{array}$ \\
\hline
\end{tabular}

Psi = psilocybin, $\mathrm{Pla}=$ placebo; Glu = glutamate; NAA = n-acetyl-aspartate; NAAG = n-acetyl aspartyl glutamate; $\mathrm{ml}=$ myoinositol; $\mathrm{tCr}$ = total creatine; $\mathrm{Gln}=$ glutamine, $\mathrm{Glx}=$ glutamate + glutamine; $\mathrm{Cr}$ = creatine; $\mathrm{PCr}=$ phosphocreatine; tCho = total choline; GSH = glutathione 


\section{Chapter 4}

Table S4. Significantly altered functional connectivity within resting state networks under the drug condition compared with placebo.

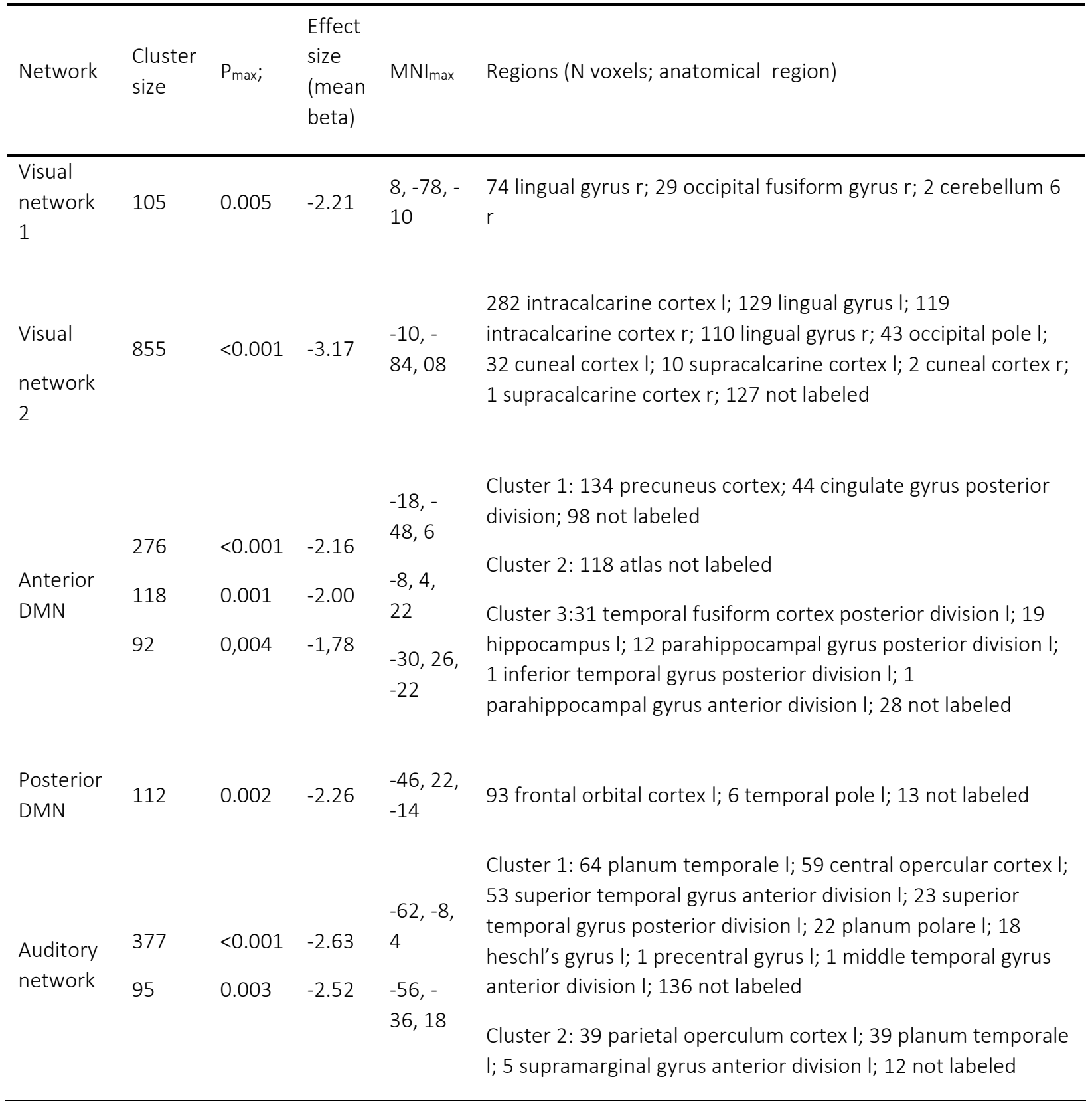


Psilocybin-induced changes in glutamate, network connectivity, and ego dissolution

Table S5. Beta values from the between-network FC analysis, demonstrating increased functional connectivity between resting state networks under psilocybin compared to placebo. Asterisks indicate significant differences between treatment conditions (psilocybin $>$ placebo, $p<0.05$, FDR, two-tailed). Vis = Visual

\begin{tabular}{|c|c|c|c|c|c|c|c|c|c|c|c|c|c|}
\hline & $\begin{array}{l}\text { Anter } \\
\text { ior } \\
\text { DMN }\end{array}$ & $\begin{array}{l}\text { Audit } \\
\text { ory }\end{array}$ & $\begin{array}{l}\text { Cerebel } \\
\text { lum }\end{array}$ & $\begin{array}{l}\text { Execut } \\
\text { ive }\end{array}$ & $\begin{array}{l}\text { Frontopar } \\
\text { ietal } 1\end{array}$ & $\begin{array}{l}\text { Frontopar } \\
\text { ietal } 2\end{array}$ & $\begin{array}{l}\text { Poster } \\
\text { ior } \\
\text { DMN }\end{array}$ & $\begin{array}{l}\text { Sensorim } \\
\text { otor } 1\end{array}$ & $\begin{array}{l}\text { Sensorim } \\
\text { otor } 2\end{array}$ & $\begin{array}{l}\text { Sensori } \\
\text { motor } 3\end{array}$ & Vis 1 & Vis 2 & $\begin{array}{l}\mathrm{Vi} \\
\mathrm{s} \\
3\end{array}$ \\
\hline $\begin{array}{l}\text { Anterior } \\
\text { DMN }\end{array}$ & & & & & & & & & & & & & \\
\hline Auditory & -0.03 & & & & & & & & & & & & \\
\hline $\begin{array}{l}\text { Cerebellu } \\
\mathrm{m}\end{array}$ & $0.15^{*}$ & 0.11 & & & & & & & & & & & \\
\hline Executive & $0.26^{*}$ & 0.08 & $0.20 *$ & & & & & & & & & & \\
\hline $\begin{array}{l}\text { Frontopar } \\
\text { ietal } 1\end{array}$ & $0.20 *$ & 0.04 & 0.10 & 0.11 & & & & & & & & & \\
\hline $\begin{array}{l}\text { Frontopar } \\
\text { ietal } 2\end{array}$ & $0.19 *$ & 0.12 & $0.14^{*}$ & 0.08 & 0.07 & & & & & & & & \\
\hline $\begin{array}{l}\text { Posterior } \\
\text { DMN }\end{array}$ & -0.01 & 0.00 & $0.14 *$ & 0.10 & $0.15^{*}$ & 0.03 & & & & & & & \\
\hline $\begin{array}{l}\text { Sensorim } \\
\text { otor } 1\end{array}$ & 0.00 & $0.19 *$ & 0.03 & $0.16^{*}$ & 0.09 & 0.12 & 0.05 & & & & & & \\
\hline $\begin{array}{l}\text { Sensorim } \\
\text { otor } 2\end{array}$ & -0.10 & -0.02 & 0.08 & 0.06 & 0.03 & 0.03 & 0.07 & 0.09 & & & & & \\
\hline $\begin{array}{l}\text { Sensorim } \\
\text { otor } 3\end{array}$ & -0.07 & $0.17^{*}$ & 0.05 & 0.06 & 0.06 & 0.13 & 0.02 & $0.20^{*}$ & -0.01 & & & & \\
\hline Vis 1 & $0.18^{*}$ & 0.11 & $0.19 *$ & $0.25 *$ & $0.21^{*}$ & $0.16^{*}$ & $0.15^{*}$ & -0.02 & 0.02 & 0.00 & & & \\
\hline Vis 2 & $0.19 *$ & 0.09 & $0.27^{*}$ & $0.21 *$ & $0.20 *$ & $0.18^{*}$ & $0.11^{*}$ & -0.01 & 0.05 & 0.01 & -0.18 & & \\
\hline Vis 3 & 0.11 & $0.13^{*}$ & 0.09 & $0.17 *$ & $0.18 *$ & $0.15^{*}$ & $0.14^{*}$ & 0.07 & 0.09 & 0.09 & -0.02 & -0.11 & \\
\hline
\end{tabular}




\section{Chapter 4}

Table S6. Overview of MRS data points that did not meet the data quality criteria check, and were subsequently not included in the analysis. Data points could be missing due to technological or participant difficulties. Once a spectra was acquired, quality was assessed and the entire spectra rejected if $\mathrm{SNR}<10$ and $\mathrm{FWHM}>0.1$. Finally, each metabolite was assessed individually, and discarded if relative CRLB $>20 \%$. Psi $=$ psilocybin.

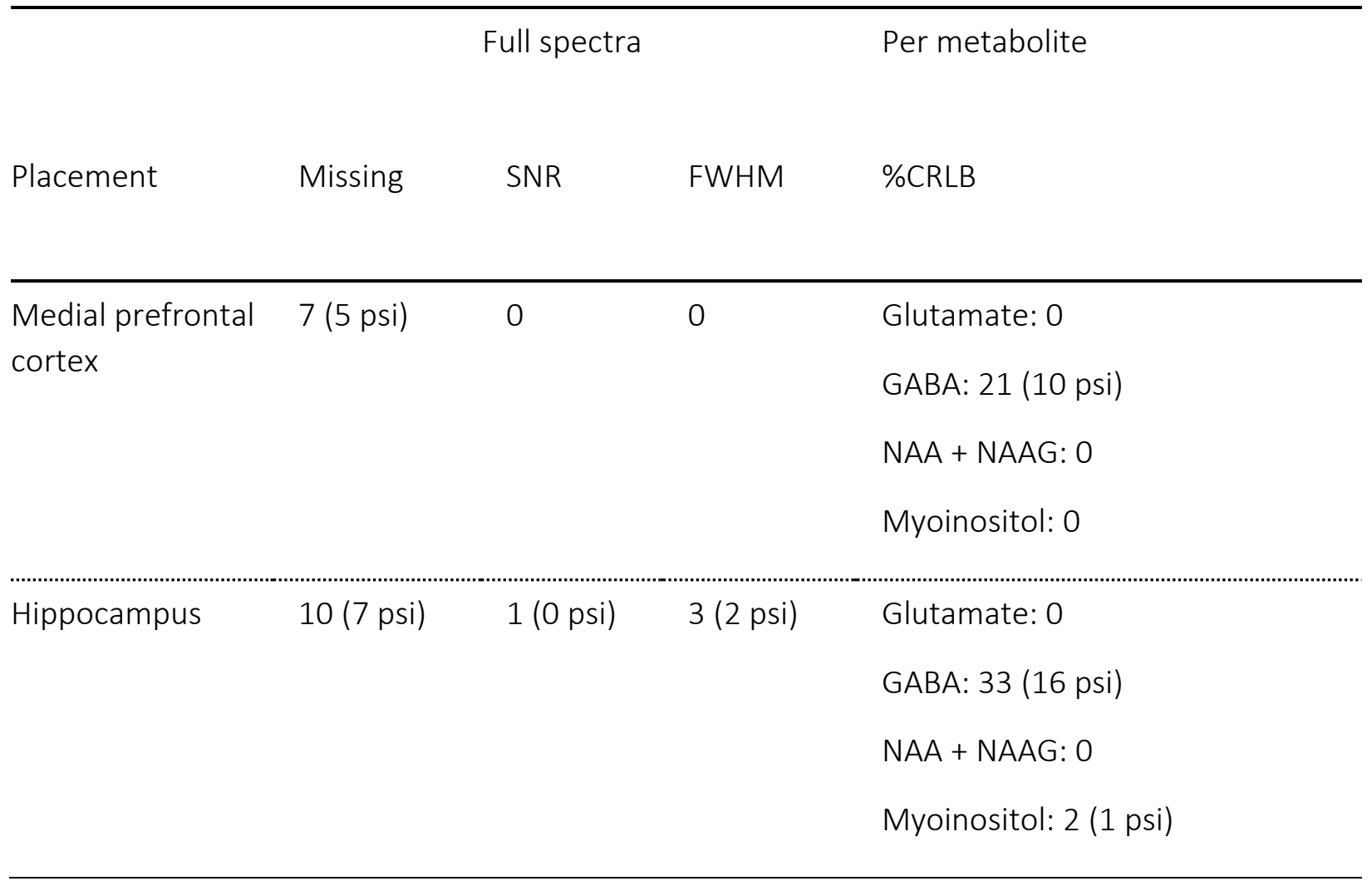




\section{References}

1 Studerus, E., Gamma, A. \& Vollenweider, F. X. Psychometric evaluation of the altered states of consciousness rating scale (OAV). PLOS ONE 5, e12412 (2010).

2 Nour, M. M., Evans, L., Nutt, D. \& Carhart-Harris, R. L. Ego-dissolution and psychedelics: validation of the ego-dissolution inventory (EDI). Frontiers in human neuroscience 10, 269 (2016).

3 Diener, E., Emmons, R. A., Larsen, R. J. \& Griffin, S. The Satisfaction With Life Scale. Journal of personality assessment 49, 71-75, doi:10.1207/s15327752jpa4901_13 (1985).

4 Pavot, W., Diener, E., Colvin, C. R. \& Sandvik, E. Further validation of the Satisfaction with Life Scale: evidence for the cross-method convergence of well-being measures. Journal of personality assessment 57, 149-161, doi:10.1207/s15327752jpa5701_17 (1991).

5 Pavot, W. \& Diener, E. The Satisfaction with Life Scale (SWL). Measurement Instrument Database for the Social Science, $<$ www.midss.ie $>$ (2013).

6 Martin, R., Schurenkamp, J., Pfeiffer, H. \& Kohler, H. A validated method for quantitation of psilocin in plasma by LCMS/MS and study of stability. International journal of legal medicine 126, 845-849, doi:10.1007/s00414-011-0652-8 (2012).

7 Marques, J. P. et al. MP2RAGE, a self bias-field corrected sequence for improved segmentation and T1-mapping at high field. Neurolmage 49, 1271-1281, doi:10.1016/j.neuroimage.2009.10.002 (2010).

8 Zhang, Y., Brady, M. \& Smith, S. Segmentation of brain MR images through a hidden Markov random field model and the expectation-maximization algorithm. IEEE transactions on medical imaging 20, 45-57, doi:10.1109/42.906424 (2001). Frahm, J. et al. Localized high-resolution proton NMR spectroscopy using stimulated echoes: initial applications to human brain in vivo. Magnetic resonance in medicine 9, 79-93 (1989).

10 Tkac, I., Starcuk, Z., Choi, I. Y. \& Gruetter, R. In vivo 1 H NMR spectroscopy of rat brain at 1 ms echo time. Magnetic resonance in medicine 41, 649-656 (1999). Klose, U. In vivo proton spectroscopy in presence of eddy currents. Magnetic resonance in medicine 14, 26-30 (1990). Barker, P. B. et al. Quantitation of proton NMR spectra of the human brain using tissue water as an internal concentration reference. NMR in biomedicine 6, 89-94 (1993).

13 Soher, B. J., Hurd, R. E., Sailasuta, N. \& Barker, P. B. Quantitation of automated single-voxel proton MRS using cerebral water as an internal reference. Magnetic resonance in medicine 36, 335-339 (1996).

Smith, S. A., Levante, T. O., Meier, B. H. \& Ernst, R. R. Computer Simulations in Magnetic Resonance. An Object-Oriented Programming Approach. Journal of Magnetic Resonance 106, 75-105 (1994).

Govindaraju, V., Young, K. \& Maudsley, A. A. Proton NMR chemical shifts and coupling constants for brain metabolites. NMR in biomedicine 13, 129-153 (2000).

Penner, J. \& Bartha, R. Semi-LASER 1 H MR spectroscopy at 7 Tesla in human brain: Metabolite quantification incorporating subject-specific macromolecule removal. Magnetic resonance in medicine, doi:10.1002/mrm.25380 (2014).

Provencher, S. W. Automatic quantitation of localized in vivo $1 \mathrm{H}$ spectra with LCModel. NMR in biomedicine 14, 260-264 (2001).

Kreis, R. Issues of spectral quality in clinical $1 \mathrm{H}$-magnetic resonance spectroscopy and a gallery of artifacts. NMR in biomedicine 17, 361-381, doi:10.1002/nbm.891 (2004).

Wilson, M. et al. Methodological consensus on clinical proton MRS of the brain: Review and recommendations. Magnetic resonance in medicine 82, 527-550, doi:10.1002/mrm.27742 (2019).

Whitfield-Gabrieli, S. \& Nieto-Castanon, A. Conn: a functional connectivity toolbox for correlated and anticorrelated brain networks. Brain connectivity 2, 125-141, doi:10.1089/brain.2012.0073 (2012).

Behzadi, Y., Restom, K., Liau, J. \& Liu, T. T. A component based noise correction method (CompCor) for BOLD and perfusion based fMRI. Neurolmage 37, 90-101, doi:10.1016/j.neuroimage.2007.04.042 (2007). 


\section{Chapter 5}

\section{Sub-acute effects of psilocybin on empathy, creative thinking, and subjective well-being}

Mason, N.L., Mischler, E., Uthaug, M.V., Kuypers, K.P.C.

Creative thinking and empathy are crucial for everyday interactions and subjective well-being. This is emphasized by studies showing a reduction in these skills in populations where social interaction and subjective well-being are significantly compromised (e.g., depression). Anecdotal reports and recent studies suggest that a single administration of psilocybin can enhance such processes and could therefore be a potential treatment. However, it has yet to be assessed whether effects outlast acute intoxication. The present study aimed to assess the sub-acute effects of psilocybin on creative thinking, empathy, and well-being. Participants attending a psilocybin retreat completed tests of creative (convergent and divergent) thinking and empathy, and the satisfaction with life scale on three occasions: before ingesting psilocybin $(N=55)$, the morning after $(N=50)$, and seven days after $(N=22)$. Results indicated that psilocybin enhanced divergent thinking and emotional empathy the morning after use. Enhancements in convergent thinking, valence-specific emotional empathy, and well-being persisted seven days after use. Sub-acute changes in empathy correlated with changes in well-being. The study demonstrates that a single administration of psilocybin in a social setting may be associated with sub-acute enhancement of creative thinking, empathy, and subjective well-being. Future research should test whether these effects contribute to the therapeutic effects in clinical populations.

Journal of Psychoactive Drugs, 2019; 51(2), 123-134 


\section{Chapter 5}

\section{Introduction}

Creativity and empathy are crucial for everyday interactions and cooperation, allowing us to adapt to an ever changing environment, and motivating our prosocial behaviors ${ }^{1}$. Interestingly, they have been found to be positively related ${ }^{2}$, and it has been further suggested that creative, flexible thinking is a prerequisite for empathy ${ }^{3}$. Importantly, previous research demonstrates a reduction in these skills in populations where social interactions and subjective well-being is significantly compromised ${ }^{4,5}$.

Creativity is a multicomponent construct, consisting of convergent (CT) and divergent thinking $(D T)^{6}$. CT is considered a process of generating a single optimal solution to a particular problem, emphasizing speed, accuracy, and logic. Contrarily, DT is a process used to generate many new ideas, in a context where more than one solution is correct. An example of the latter is a brainstorming session, where generating as many innovative ideas or solutions on a particular issue as possible is the ultimate goal ${ }^{7}$. Although both CT and DT are important in creative activities, DT has been suggested to be a more useful estimate of the potential for creative thought in daily life ${ }^{8}$.

Emotional, or affective, empathy (EE) refers to the sharing of emotions, or the ability to feel what another person is feeling. In contrast, cognitive empathy (CE) refers to mental perspective taking, or recognizing and understanding what another person is feeling ${ }^{9}$. Previous research suggests a specificity of the two constructs ${ }^{10}$, in that emotional empathy may be dependent on state variables, whereas cognitive empathy requires a (trait) ability to identify another's emotions ${ }^{11,12}$.

Both creative, flexible thinking and empathy deficits have been found in stress-related psychopathologies like depression, anxiety disorders, and post-traumatic stress disorder (PTSD) ${ }^{13-}$ 20. Hallmarks of these disorders are repetitive and rigid patterns of negative and compulsive thoughts, together with social difficulties and impaired empathic abilities 5,18,21-24The latter of which is most evident in depression, and has been suggested to contribute to more pronounced symptoms ${ }^{14,15}$.

Importantly, previous research has found that these processes demonstrate plasticity and can be enhanced by interventions, such as therapy programs, hormone administration, and mindfulness induction, as well as (positive) changes in mood11,15,16,25-29. However, these interventions typically target only one of the two mentioned processes. Thus, by finding treatments that promote both processes, individual deficits could be further decreased, potentially enhancing well-being and quality of life. 
Anecdotal evidence and (quasi-) experimental studies suggest that a single administration of a psychedelic drug like ayahuasca, LSD, and psilocybin can enhance creative, flexible thinking and emotional empathy in the neuro-typical population ${ }^{12,30-37}$. Furthermore, clinical studies have found that administration of psilocybin can induce long-lasting positive psychological changes, such as symptom remission and enhancement of well-being, in clinical populations ${ }^{38-40}$. However, the persistence of effects on creativity and empathy, and the relationship with subjective wellbeing, has yet to be assessed.

The present study was therefore designed to assess the sub-acute effects of psilocybin on creative thinking, empathy, and subjective well-being. Based on the aforementioned literature, it was hypothesized that divergent and convergent thinking, emotional empathy, and satisfaction with life, would be enhanced sub-acutely with no effect on cognitive empathy ${ }^{12}$. Furthermore, based on previous clinical research demonstrating reductions in creativity and empathy in individuals with compromised well-being, it was hypothesized that enhancements in such processes would correlate with increased satisfaction with life. Finally, as psilocybin has shown to induce long-lasting positive increases in well-being, it was hypothesized that participants with previous psilocybin experience would have a higher baseline satisfaction with life score, compared to those who are psilocybin-naïve.

\section{Methods}

\section{Participants}

Participants were volunteers attending psilocybin retreats in the Netherlands, organized by the Psychedelic Society UK. In total, 55 participants (26 female) consented after goals and methods of the study were explained. Most participants were from Europe (80\%), while the rest were from North America (7.3\%), Africa (3.6\%), Central America (1.8\%), and Asia (1.8\%), or undisclosed (5.4\%). The highest completed level of education was graduate school (41.8\%), undergraduate school (41.8\%), secondary school (7.3\%), or undisclosed (9.1\%). Mean (SD) participant age was 34.8 (8.9).

About half $(52.7 \%)$ of the participants had used psilocybin before, and $49.1 \%$ had previously used a psychedelic other than psilocybin (LSD, ayahuasca, or DMT). Motivations for attending the retreat included "to understand myself" (83.6\%), "curiosity" (80\%), "to resolve problems" (49.1\%), and "other" (18.2\%). For 69.1\% of the participants, this was the first time taking a psychedelic in a retreat setting.

The study was conducted in accordance with the Declaration of Helsinki and subsequent amendments concerning research in humans and was approved by the Ethics Review Committee of Psychology and Neuroscience (175_03_2017). Participation was voluntary and no incentive to participate was provided. All volunteers gave their written informed consent to participate. 


\section{Study procedure}

\section{Psilocybin retreats}

Prior to participation in the retreat, personal intakes were done by the facilitators, which included screening for (and excluding) individuals with psychiatric disorders or taking psychiatric medications, and medical factors like high blood pressure.

The setting in which psilocybin was taken was the same throughout all of the retreats. Participants stayed in a large house set in nature, hosted by at least two or more experienced psilocybin facilitators. They arrived the evening before psilocybin administration, and were able to get acquainted with each other, the facilitators, and the schedule of the retreat. The next day, participants received the psilocybin-containing truffles around noon, in a tea form. After ingestion, participants were instructed to stay on the premises, and were able to do what they wanted, as long as they did not disturb other participants. Facilitators provided music, tools to draw and/or write, and food. In the evening, all participants and facilitators came back together as a group. The next morning, all participants had breakfast together, and had a closing group meeting.

\section{Psilocybin}

Participants ingested the truffles in a tea form, guided by the facilitators. To do this, the truffles were crushed, and boiling hot ginger tea was added. After infusing for a few minutes, the participants drank the tea, and were subsequently free to add more water and repeat the process $2-3$ times. Afterwards participants could eat the remaining truffle contents in the cup.

Previous experimental studies have demonstrated that subjective alterations after psilocybin intake begin 20-40 minute following administration, peak around 60-90 minutes, and subside by 6 hours post intake ${ }^{41}$. However anecdotal reports suggest that when ingested in tea form, subjective alterations are felt more quickly, and for a shorter amount of time ${ }^{42}$.

\section{Study procedure}

Creativity, empathy, and well-being assessments were taken on three separate occasions: at baseline (the evening before ingesting psilocybin), the morning after ingesting psilocybin, and 7 days after ingesting psilocybin. Participants completed pre- and post-psilocybin assessments at the retreat, with the investigators present. The third assessment was completed online. The total amount of psilocybin truffles taken by each participant was recorded, and a sample of the truffles was taken to determine concentrations of psilocybin and its metabolite psilocin. The German Central Customs Authority determined the contents of psilocin and psilocybin after freeze-drying the truffles using a previously described HPLC method ${ }^{43}$. 


\section{Picture Concept Task}

In order to assess creativity, the picture concept task (PCT) was used ${ }^{32}$. The PCT consists of 17 stimuli, each containing between 4 and 12 color pictures shown in a matrix of $2 \times 2,2 \times 3,3 \times 3$, or $3 \times 4$. Participants were instructed to find an association between one of the pictures in each row. Specifically, they were asked first to provide the correct solution as there is only one correct answer. The number of correct answers served as the dependent measure of convergent thinking. In order to assess divergent thinking, participants were asked to provide as many alternative answers as possible. This is the regular instruction included in measures of divergent thinking, and it is used to calculate several parameters, i.e., fluency, originality, and the ratio of both, which reflect quantity and quality of divergent thinking. Fluency is defined as the number of alternative associations. The second parameter, i.e., originality, is calculated by evaluating the originality of the alternative association relative to those provided by all other participants in a session. Alternative answers that were uniquely reported by a single participant received an originality score of 2 . Answers that were shared with a single participant were valued as 1 , and answers that were shared by 3 or more participants were rated zero. Mean originality (creativity) scores and ratio originality scores weighed for fluency (originality/fluency) were used as measures of divergent thinking. Three parallel versions of the PCT were used at baseline and the two follow-up measures after the retreat to avoid learning effects; participants had 30 seconds per stimulus. Previous studies have found the PCT to be sensitive to the effects of psychedelics $^{32,37,44}$.

\section{Multifaceted Empathy Test}

The multifaceted empathy test (MET) consists of 40 pictures of people in various emotional states, with $50 \%$ being positive and $50 \%$ negative ${ }^{45}$. To assess cognitive empathy (CE), participants were asked to select the emotion word, out of four words, that matched the depicted emotion. To assess emotional empathy (EE), participants were asked to rate on a scale from 1 to 9 'how aroused does this picture make you feel' (implicit EE) and 'how concerned do you feel for this person' (explicit EE). The number of correctly classified pictures (CE) and the implicit EE and explicit EE ratings per valence and averaged across valences were used as dependent variables. Previous validity and reliability analysis of the MET have shown to be in the good to highly satisfactory range ${ }^{45}$, and previous studies have found it to be sensitive to the effects of psychedelics ${ }^{12,34,46-49}$.

\section{Satisfaction with Life Scale}

The Satisfaction with Life Scale (SWLS) is a 5-item questionnaire designed to measure global cognitive judgments of satisfaction with one's life ${ }^{50}$, and has been used to measure the life 
satisfaction component of subjective well-being ${ }^{50,51}$. Individuals answer each item on a Likertscale ranging from 1 (strongly disagree) to 7 (strongly agree). The total score is then obtained by summing the ratings form each item. Sample items include "In most ways my life is close to my ideal" and "If I could live my life over, I would change almost nothing". The minimum possible score is 5, and a maximum possible score of 35, with a score of 5-19 defined as dissatisfied to below average life satisfaction, 20-24 defined as average life satisfaction, and 29-35 defined as high to very high life satisfaction ${ }^{52}$. The scale has previously been shown to be a valid and reliable measure of life satisfaction ${ }^{53}$.

\section{Psilocybin experience}

The morning after ingesting psilocybin, participants were asked to retrospectively rate the intensity of various aspects of the acute psilocybin experience using ten visual analog scales (VASs). The VASs were $10 \mathrm{~cm}$ horizontal lines, with a bottom anchor of "not more than usually" and a top anchor of "much more than usually". These items have previously been shown to be sensitive to the acute effects of psilocybin ${ }^{54}$.

\section{Statistics}

Statistical analysis was conducted in IBM SPSS Statistics 24 using a linear repeated measures model analysis that included Session (three levels: baseline, morning after psilocybin, and 7 days after psilocybin) as a within-subject factor. Due to small sample size at the 7 day follow-up, a separate linear repeated measures model analysis was done for each outcome variable. Similarly, as for the MET, if a main effect of Session was found on emotional or cognitive empathy, two further analyses were done, separating valence-specific (positive or negative emotion) responses.

The covariance structure was chosen according to best fit and could vary across outcome variables. Different covariance structures used included compound symmetry heterogenous (CSH) and first lag autoregressive (AR1) structures. If a main effect of Session was found, separate contrasts were performed between baseline and the follow-up sessions with Bonferroni adjustment for multiple comparisons.

In order to test whether people with previous psilocybin experience differed from psilocybin naïve participants on outcome measures, a further mixed model analysis was conducted, with session (three levels: baseline, morning after psilocybin, and 7 days after psilocybin) as a within-subject factor and previous experience with psilocybin (two levels: yes, no) as a between subjects factor, though only for outcome parameters which showed a significant main effect of Session in the first analysis. The analysis was performed to determine whether subacute effects of psilocybin differed between experienced versus naïve psilocybin users. 
In order to investigate the association between cognitive (creativity and empathy) and subjective (well-being) outcome parameters, Pearson's correlations were carried out using baseline change scores (Morning after - baseline; 7 days after - baseline).

Psilocybin experience ratings were analyzed separately using one-sample $t$ tests comparing the scores on each VAS after psilocybin versus a zero distribution since previous studies have shown that placebo scores are low, not rising above a 0 in a scale from 0 to $100{ }^{55}$.

The alpha criterion level of significance for all analyses was set at $p=0.05$.

\section{Results}

In total, 55 participants completed parts of the test battery at baseline, 50 completed parts of the test battery the morning after taking psilocybin, and 22 completed parts of the test battery 7 days after taking psilocybin. Incomplete or missing test batteries were due to time constraints and/or participant drop out.

\section{Psilocybin}

The truffle sample (15 grams; Psilocybe Hollandia) contained $1.9 \mathrm{mg}$ of psilocybin and $10.5 \mathrm{mg}$ of psilocin. Participants ingested an average (SD) 34.2 (8.9) grams of truffles throughout the day. Once ingested, psilocybin is quickly metabolized to psilocin at a calculation factor of 0.719 , resulting in a final (average) psilocin consumption of $27.1 \mathrm{mg}$.

\section{Picture Concept Test}

Convergent thinking. Analysis revealed a significant main effect of Session $\left(F_{2,37.48}=5.94, p=.01\right)$ on the number of correct associations (see Figure 1D). Compared to baseline, participants were able to identify a higher number of correct associations 7 days after ingesting psilocybin $(p=.01 ; d=.46)$. There were no significant effects of psilocybin on convergent thinking the morning after taking psilocybin.

Divergent thinking. Analysis revealed a significant main effect of Session on Fluency $\left(F_{2,38.22}=5.27\right.$, $p=.01$ ) and Originality $\left(F_{2,45.09}=7.23, p=.002\right)$ (see Figure $\left.1 \mathrm{~A}, \mathrm{~B}\right)$. Compared to baseline, participants were able to come up with more associations ( $p=.01 ; d=.47)$, and had a higher originality score $(p=.001 ; d=.55)$, the morning after taking psilocybin. There was no significant effect of session on Ratio $\left(F_{2,42.44}=2.25, p=.12\right)$. Furthermore, there were no significant effects of psilocybin on Fluency, Originality, or Ratio, 7 days after taking psilocybin. 

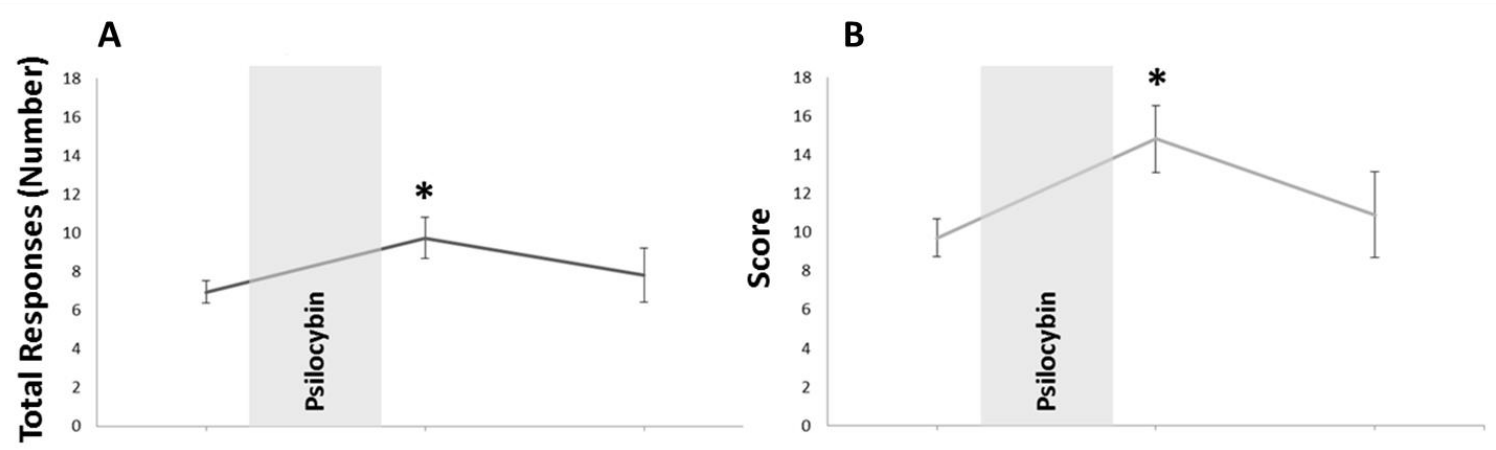

C

D
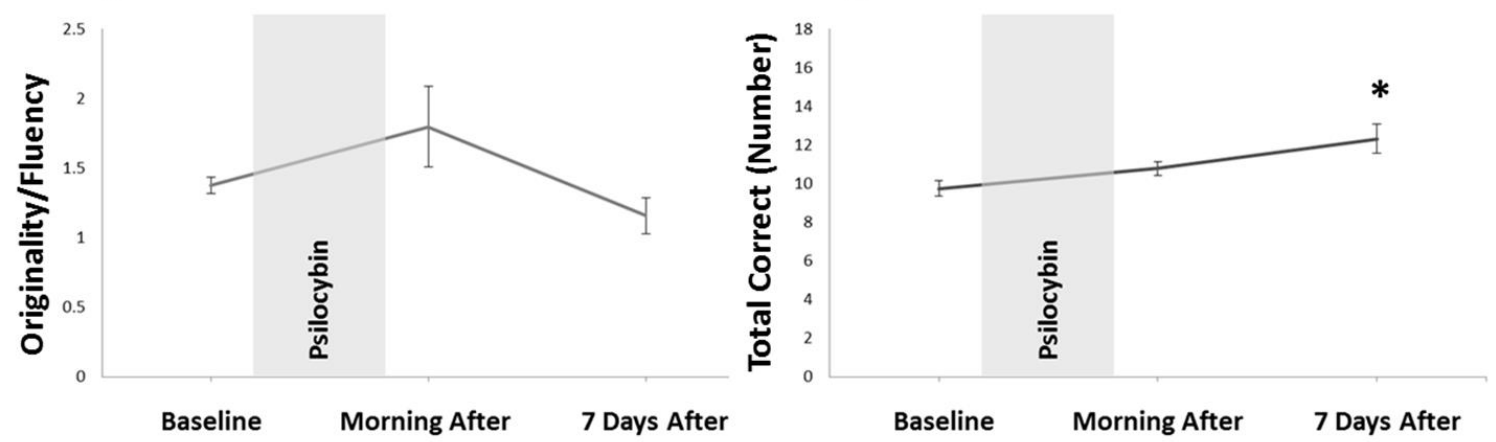

Figure 1. Mean ( $\pm S E$ ) outcome variables of divergent and convergent thinking, measured before, the morning after, and 7 days after psilocybin ingestion. Panels $A$ to $C$ depict outcome variables of divergent thinking; $(A)$ fluency, $(B)$ originality, $(C)$ the ratio, and panel (D) depicts the outcome variable of convergent thinking. $\left({ }^{*} p<.05\right)$

\section{Multifaceted Empathy Test}

Cognitive empathy. There was no significant effect of Session on cognitive empathy $\left(F_{2,23.26}=3.18\right.$, $p=.06)$; participants were able to recognize about 22 emotions on average in the three sessions (see Figure 2C,D).

Emotional empathy. Analysis revealed a significant main effect of Session $\left(F_{2,48.31}=4.29, p=.02\right)$ on average Explicit EE (see Figure 2B). Compared to baseline, participants felt more concern for people depicting emotions the morning after ingesting psilocybin ( $p=.02 ; d=.45)$. When assessing valence-specific responses, analysis revealed a significant main effect of Session $\left(F_{2,38.04}=5.87\right.$, $p=.01$ ) on concern ratings of negative emotions; compared to baseline, individuals concern for negative emotions was increased the morning after taking psilocybin $(p=.01 ; d=.49)$ without effects on day 7 . There was no significant effect of Session $\left(F_{2,51.42}=2.58, p=.09\right)$ on concern ratings of positive Explicit EE.

Analysis revealed a significant main effect of Session $\left(F_{2,23.71}=10.64, p=.001\right)$ on average Implicit EE ratings (see Figure $2 \mathrm{~A}$ ). Compared to baseline, participants felt more aroused by the 
emotional content of the stimuli the morning after ingesting psilocybin $(p<.001 ; d=.71)$. When assessing valence-specific responses, analysis revealed a significant main effect of Session on both positive $\left(F_{2,25.72}=7.89, p=.002\right)$ and negative $\left(F_{2,19.18}=4.93, p=.02\right)$ emotions. Separate contrasts indicated that compared to baseline, individuals' arousal to both positive $(p=.001 ; d=.61)$ and negative ( $p=.01 ; d=.48$ ) emotions was higher the morning after ingesting psilocybin. Furthermore, implicit arousal to negative stimuli, but not positive stimuli remained increased 7 days after ingesting psilocybin ( $p=.05 ; d=.41)$.
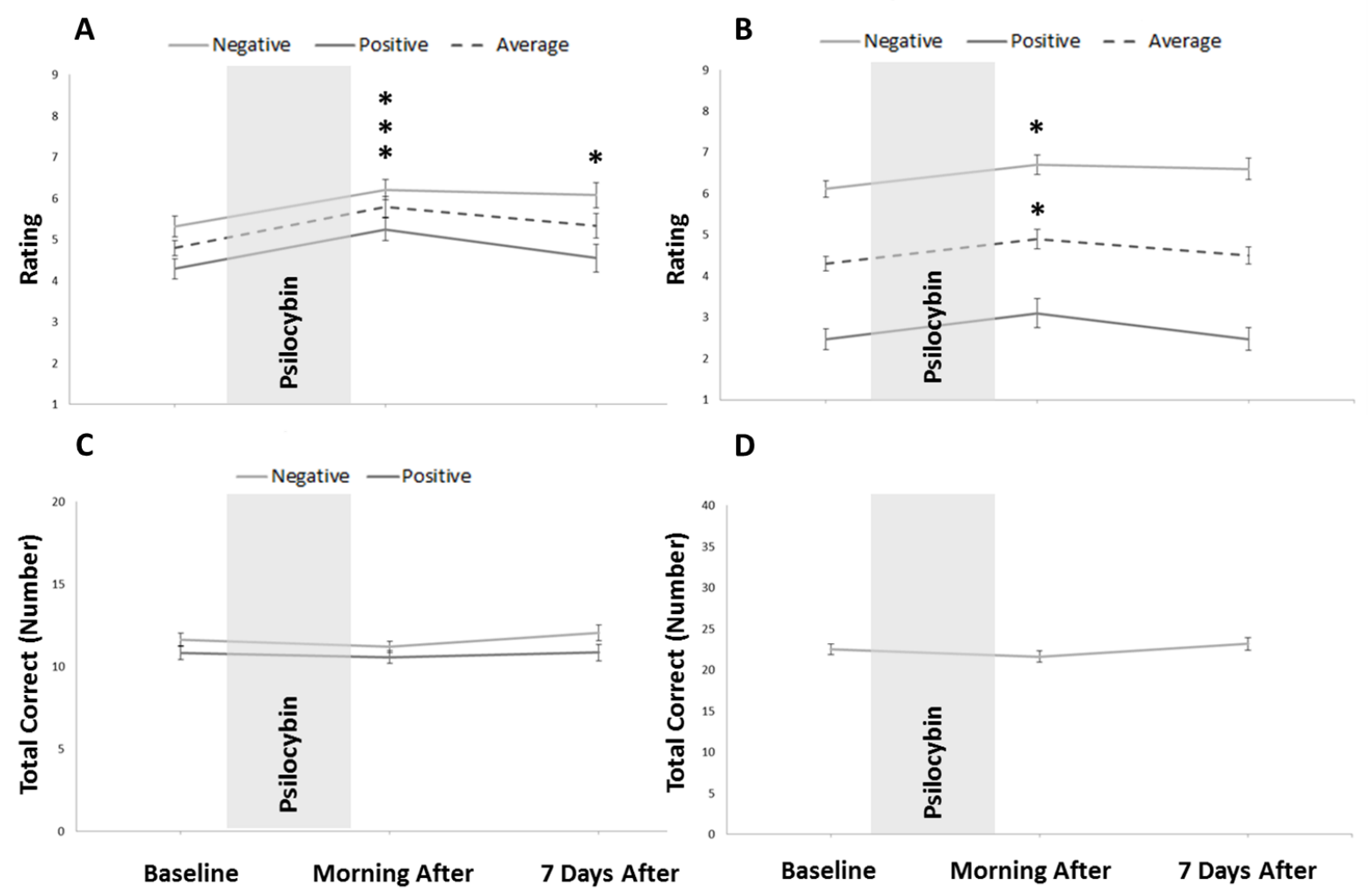

D

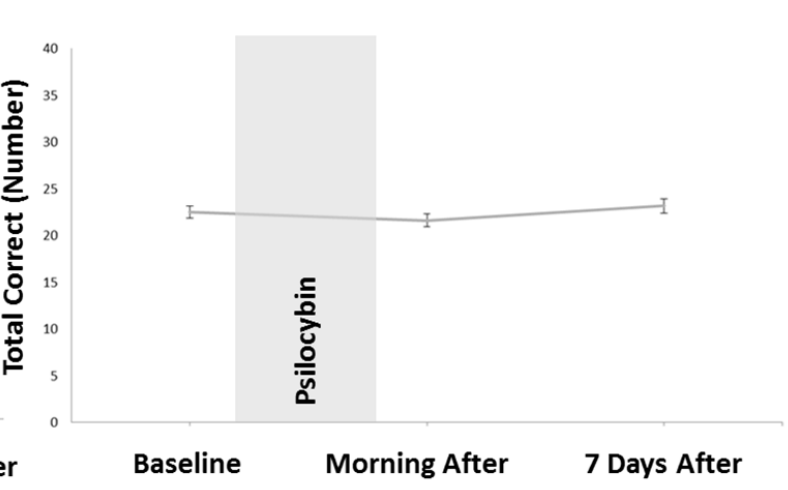

Figure 2. Mean ( $\pm S E)$ outcome variables of implicit emotional empathy $(A)$, explicit emotional empathy (B), and cognitive empathy (C; total (D)), measured before, the morning after, and 7 days after psilocybin ingestion $\left({ }^{*} p<.05\right)$

\section{Satisfaction with Life Scale}

Analysis revealed a significant main effect of Session $\left(F_{2,48.31}=17.83, p<.001\right)$ on individuals' responses to the SWLS (see Figure $3 \mathrm{~A}$ ). Contrasts indicated that, compared to baseline, satisfaction with life significantly increased both the morning after $(p<.001 ; d=.77)$ and 7 days after $(p=.001$; $d=.50$ ) ingesting psilocybin. 


\section{Previous experience with psilocybin}

Twenty-nine participants reported previously using psilocybin, whereas 21 reported being psilocybin naïve, and 5 chose not to disclose their previous drug use history. The latter were thus not included in this analysis. Analysis revealed a significant main effect of Session $\left(F_{2,52.44}=16.768\right.$, $p<.000)$ and Psilocybin experience $\left(F_{1,46.93}=5.39, p=.025\right)$ on individuals' response to the SWLS (Figure 3B). Contrasts indicated that those who had previous experience with psilocybin reported a significantly higher quality of life at baseline compared to those who were psilocybin naïve ( $p=.012 ; d=.39)$. The sub-acute effects of psilocybin did not differ between participants who had used psilocybin vs those who had not for any other outcome measures.

A

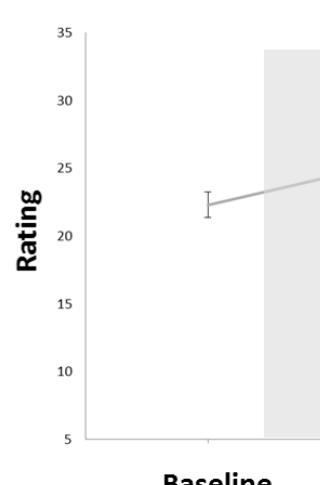

Baseline

\begin{abstract}
Morning After
\end{abstract}
7 Days After

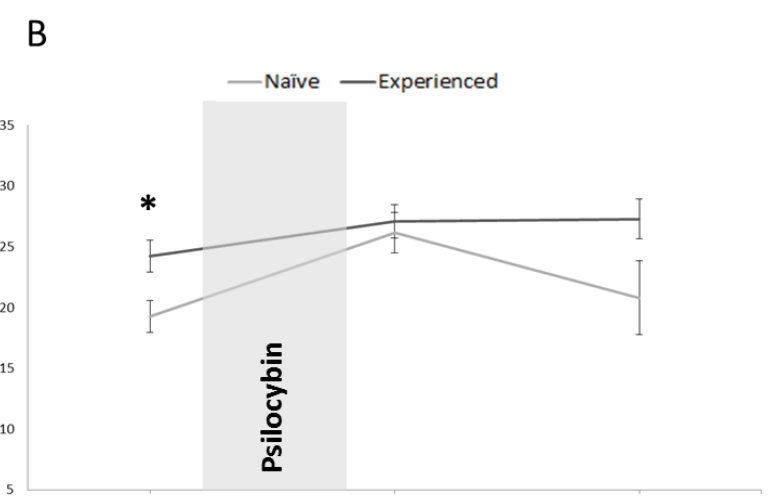

Baseline
Morning After
7 Days After

Figure 3. Panel A depicts the total group mean ( \pm SE) ratings of satisfaction with life measured before, the morning after, and 7 days after psilocybin ingestion. In panel B a second analysis was performed to assess differences in satisfaction with life between those who had previous experience with psilocybin (experienced) and those who had not (naive). Mean ( \pm SE) ratings per group are shown. $\left({ }^{*} p<.05\right)$

\section{Correlations}

Analysis showed medium to strong positive correlations between changes in implicit and explicit EE towards a positive stimulus, and changes in satisfaction with life. Specifically, as changes in arousal to pictures of people in positive mood states increased, changes in satisfaction with life also increased, when comparing baseline to both the morning after psilocybin intake $\left(r_{s}=.43, p=.05\right)$ and 7 days after intake $\left(r_{s}=.61, p=.02\right)$. Furthermore, as changes in ratings of concern for people in various emotional states increased between baseline and the morning after intake, ratings of satisfaction with life increased between baseline and 7 days after intake $\left(r_{s}=.57, p=.03\right)$. Finally, as changes in ratings of concern for people in positive mood states increased, changes in quality of 
life increased between baseline and 7 days after psilocybin intake $\left(r_{s}=.58, p=.03\right)$. No significant correlations were found between outcome variables of the PCT and ratings of quality of life, or between variables of the PCT and the MET.

\section{Psilocybin experience}

Mean (SE) ratings on the different VAS items after psilocybin are shown in Figure 4. The onesample $t$ tests showed psilocybin-induced significant increases in all VAS items ( $t_{49}=14.56-23.73$; $p \leq .000 ; d=2.05-3.35)$.

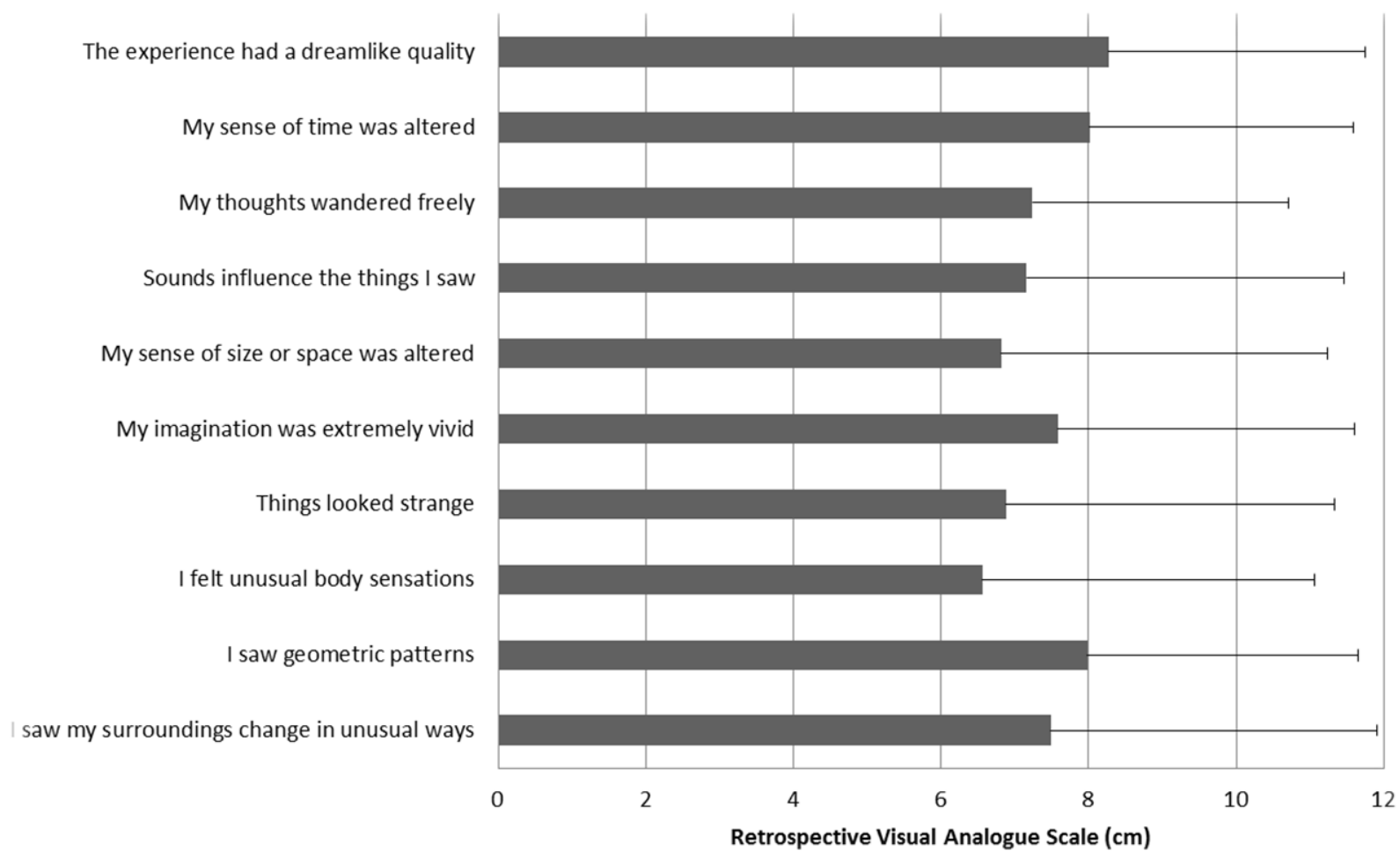

Figure 4. Mean ( $\pm S E$ ) of visual analogue scale item scores, on a $10 \mathrm{~cm}$ scale. Scores are retrospective of the psilocybin experience.

\section{Discussion}

The present study demonstrates the first attempt to assess the sub-acute influence of psilocybin on creative thinking, empathy, and their relationship with well-being. Utilizing a naturalistic approach, significant sub-acute enhancements of outcome measures of divergent thinking (DT), convergent thinking (CT), emotional empathy $(\mathrm{EE})$, and satisfaction with life, after ingestion of psilocybin at a psychedelic retreat, were demonstrated relative to baseline. Correlational analyses 
suggest a positive relationship between sub-acute enhancements in emotional empathy and satisfaction with life.

Findings demonstrate a time- and construct-related differentiation of effects of psilocybin on creativity; whereas DT was shown to increase the morning after ingesting psilocybin relative to baseline, CT was unaffected. Seven days after ingestion, DT performance returned to baseline whereas CT was enhanced relative to baseline. Previous research has shown that ayahuasca, a South American plant tea consisting of the similar acting 5- $\mathrm{HT}_{2 \mathrm{~A}}$ agonist N,N-Dimethyltryptamine (DMT), acutely enhanced DT and impaired CT in individuals participating in an ayahuasca ceremony ${ }^{32}$. Furthermore, a similar study demonstrated persisting sub-acute enhancements of $\mathrm{CT}$, one month after participation in an ayahuasca ceremony ${ }^{37}$. Taken together, our findings suggest that whereas psychedelic-induced enhancements of DT outlast the acute phase, decrements in CT do not. This discrepancy in acute versus sub-acute effects after a psychedelic is interesting and important in light of therapeutic implications, as both DT and CT are important components in the therapeutic process. Specifically, it has previously been suggested that DT can enhance psychological flexibility by allowing individuals to generate new, more effective strategies, which facilitate adaptive interpretations and coping abilities ${ }^{56}$. Consequently, the ability of psilocybin to enhance DT sub-acutely could help patients to relive events, recall various associations, and consider their situation from another perspective ${ }^{57-59}$. Longer-term effects on CT could then be suited in a subsequent integration session in which patients discuss their acute experiences and decide on a strategy to help them cope with intensive emotions ${ }^{32}$.

Findings demonstrate a time-, component-, and valence-differentiation of effects of psilocybin on empathy; whereas explicit and implicit emotional empathy (EE) were shown to increase the morning after psilocybin use, cognitive empathy (CE) was unaffected. Furthermore, enhancement in implicit EE to pictures depicting negative emotions persisted until 7 days after use. The sub-acute enhancements in emotional empathy are in line with previous studies assessing acute effects of psilocybin, as well as other serotonergic psychedelics like LSD and MDMA, on empathy. Specifically, psilocybin, LSD, and MDMA have been found to enhance emotional empathy on the $\mathrm{MET}^{12,34,46-49}$, without impairing cognitive empathy. Taken together, findings suggest that psilocybin (sub)-acutely increases individuals' ability to feel what other people are feeling, without affecting individuals' ability to understand what other people are feeling. Furthermore, psilocybin-induced increments in arousal to the (negative) emotion of others outlast induced increments in the ability to feel for others.

Self-rated satisfaction with life increased after psilocybin ingestion compared to baseline, both the morning after and 7 days after use. Specifically, at baseline participants reported a mean $( \pm$ SE) score of $22.3(.96)$, a morning after score of 26.5 (1.03), and 7 day after score of 25.6 (1.11). 
Interpretation of the scale states that a score between 20-24 reflects an "average" satisfaction with life, defined by general satisfaction, but with a desire for improvement in major domains (work/school, family, friends, etc...) ${ }^{52}$. A score of 25-29 is considered a "high" score, suggestive of an enjoyable life and satisfaction in the major domains. Based on interpretation scores, results suggest that psilocybin ingestion increased individuals' satisfaction with life from "average" to "high", until at least 7 days after use. This finding is consistent with previous studies showing acute $^{49}$, sub-acute 37,60 , and long-term ${ }^{61-68}$ positive psychological changes after psychedelic use. Due to these suggested persisting effects, and the fact that our sample included both psilocybin naïve and psilocybin experienced participants, we assessed whether there was a relationship between previous experience with psilocybin and baseline satisfaction with life scores. In line with the aforementioned research, it was found that those who had previously used psilocybin reported higher satisfaction with life at baseline compared to those who were psilocybin naïve, however, this was not related to differences between groups on performance measures. Altogether, results add to the growing body of literature suggesting beneficial subjective psychological effects of psychedelic use outlasting acute intoxication.

It was hypothesized that changes in empathy and creativity would correlate with changes in satisfaction with life. Correlational analysis suggested a positive relationship between changes in satisfaction with life and changes in implicit emotional empathy ('arousal'). Morning after change score increases in arousal in response to positive emotions correlated with morning after and 7 day after change score increases in subjective ratings of satisfaction with life. This relationship could be due to an increase in positive empathy, the phenomenon of sharing and understanding other's positive emotions ${ }^{69}$. Specifically, it has been demonstrated that the ability to share, celebrate, and enjoy others' positive emotions correlates with increased prosocial behavior and well-being. Furthermore, it has been hypothesized that enhanced positive empathy may even increase subjective well-being ${ }^{69}$, although directionality has not been established. Our results could provide limited evidence for directionality, as they demonstrate that an earlier (morning after) increase in positive arousal strongly correlates with a later (7 days after) increase in well-being. However, future research should more formally assess a causal relationship between (positive) empathy and well-being. Alternatively, another explanation for this relationship stems from a previous study that demonstrated that psilocybin acutely enhances response bias towards positive emotions ${ }^{70}$. Thus, individuals may feel more "aroused" when viewing positive emotions because they are in fact paying more attention to them. The shift of emotional bias to positive emotions is a proposed mechanism of antidepressant effects ${ }^{70}$, increasing individuals psychological well-being. Importantly, previous studies have implicated empathic deficits in symptom severity of depression ${ }^{14,71}$. Therefore, it could be hypothesized that enhancing implicit 


\section{Chapter 5}

emotional empathy could decrease depressive symptom severity by shifting emotional biases towards positive emotions.

Correlational analysis also suggested a relationship between changes in satisfaction with life and changes in explicit emotional empathy. Morning after change score increases in concern for emotional pictures and 7 day after change score increases in concern for negative pictures correlated with 7 day after change score increases in subjective quality of life. This is consistent with previous findings of a relationship between empathic concern and well-being ${ }^{72,73}$. Interestingly, it has also been suggested that mindfulness, namely non-judgmental, present moment awareness, increases empathic concern ${ }^{74}$. A growing body of evidence suggests that mindfulness capabilities promote subjective well-being ${ }^{75-77}$. Furthermore, previous research has found that the psychedelic, ayahuasca, post-acutely enhances non-judgmental processing of experiences ${ }^{78,79}$ and awareness ${ }^{37}$. As short-term increases in concern for emotional states of others correlated with longer-term increases in subjective quality of life, it could be hypothesized that psychedelic-induced enhancements of mindfulness processes mediate alterations in empathic concern, which influence changes in satisfaction with life and well-being.

A relationship was not found between changes in DT or CT and satisfaction with life. However, the previously discussed therapeutic implications of enhanced creativity are still relevant. Thus it could be hypothesized that, while not directly increasing subjective well-being, enhancements in constructs of creativity open up a "window of opportunity" where therapeutic interventions could prove more effective.

The current study is not without its limitations. The small sample size restricts the generalizability of the data, and high drop-out rates result in loss of statistical power at the 7 day follow-up. Additional factors that restrict generalizability of the data to the normal population include the selection bias of the sample. Namely, individuals chose to attend the retreat for various reasons, previously stated in this paper. Due to the non-random sample, as well as the lack of placebo-control, it could be argued that sub-acute enhancements are due to uncontrolled factors such as psychological expectations, or environment that the drug is taken in. Previous research and anecdotal evidence has shown that both factors, termed set and setting, play an important role in the outcome of a psychedelic experience ${ }^{67,80}$. However, previous studies demonstrating significant enhancement of psychedelics on creativity, empathy, and positive psychological outcomes have been found in a wide range of settings, including clinical $^{38,61-63,81}$, experimental 12,34,46-49, and naturalistic environments $32,37,60,64,68$. Taken together, this is a strong indicator that present study effects are directly related to drug intake. That being said, future placebo-controlled experimental studies could ideally control for the potential influence of nonpharmacological factors. Finally, future longitudinal clinical research into the therapeutic 
mechanisms of psilocybin could further assess the role of creativity and empathy in symptom alleviation in the pathological population.

In conclusion, the present study demonstrates that psilocybin, taken in a naturalistic setting, promotes constructs of creativity and empathy, and enhances subjective well-being. These findings highlight the possible underlying role of enhanced creativity and empathy in the therapeutic potential of psychedelics. Importantly, effects outlast the acute state, potentially opening up a "window of opportunity" where therapeutic interventions could prove more effective. Thus, findings add further support to growing evidence suggesting psychedelics may hold therapeutic value for treating stress-related mood disorders.

\section{Acknowledgements}

The authors would like to acknowledge and thank Stefana Bosse and the Psychedelic Society UK for their collaboration, and permission to collect data at their experience weekends. They would also like to thank all participants for their time and effort.

\section{Funding and Disclosure}

The authors declare no conflict of interest 


\section{Chapter 5}

\section{References}

1 Decety, J., Bartal, I. B. A., Uzefovsky, F. \& Knafo-Noam, A. Empathy as a driver of prosocial behaviour: highly conserved neurobehavioural mechanisms across species. Philosophical Transactions of the Royal Society B: Biological Sciences 371, 20150077, doi:10.1098/rstb.2015.0077 (2016). Carlozzi, A. F., Bull, K. S., Eells, G. T. \& Hurlburt, J. D. Empathy as Related to Creativity, Dogmatism, and Expressiveness. The Journal of Psychology 129, 365-373, doi:10.1080/00223980.1995.9914974 (1995). Eslinger, P. J. Neurological and neuropsychological bases of empathy. European neurology 39, 193-199 (1998). Neumann, M. et al. Empathy decline and its reasons: a systematic review of studies with medical students and residents. Academic medicine 86, 996-1009 (2011). Beck, A. T. Depression: Clinical, experimental, and theoretical aspects. (University of Pennsylvania Press, 1967). Guilford, J. P. The nature of human intelligence. (McGraw-Hill, 1967). Colzato, L., Szapora, A. \& Hommel, B. Meditate to Create: The Impact of Focused-Attention and Open-Monitoring Training on Convergent and Divergent Thinking. Frontiers in Psychology 3, doi:10.3389/fpsyg.2012.00116 (2012). Runco, M. A. \& Acar, S. Divergent Thinking as an Indicator of Creative Potential. Creativity Research Journal 24, 66-75, doi:10.1080/10400419.2012.652929 (2012).

9 Deutsch, F. \& Madle, R. A. Empathy: Historic and current conceptualizations, measurement, and a cognitive theoretical perspective. Human development 18, 267-287 (1975). Davis, M. H. A multidimensional approach to individual differences in empathy. (1980). Hurlemann, R. et al. Oxytocin enhances amygdala-dependent, socially reinforced learning and emotional empathy in humans. The Journal of neuroscience : the official journal of the Society for Neuroscience 30, 4999-5007, doi:10.1523/jneurosci.5538-09.2010 (2010). Pokorny, T., Preller, K., Kometer, M., Dziobek, I. \& Vollenweider, F. Effect of psilocybin on empathy and moral decisionmaking. International Journal of Neuropsychopharmacology 20, 747-757 (2017).

Palm, K. M. \& Follette, V. M. The roles of cognitive flexibility and experiential avoidance in explaining psychological distress in survivors of interpersonal victimization. Journal of Psychopathology and Behavioral Assessment 33, 79-86 (2011).

Cusi, A. M., Macqueen, G. M., Spreng, R. R. \& McKinnon, M. C. Altered empathic responding in major depressive disorder: relation to symptom severity, illness burden, and psychosocial outcome. Psychiatry research 188, 231-236, doi:10.1016/j.psychres.2011.04.013 (2011). Donges, U. S. et al. Reduced awareness of others' emotions in unipolar depressed patients. The Journal of nervous and mental disease 193, 331-337 (2005).

( Disorder. Journal of Behavior Therapy and Experimental Psychiatry 45, 208-216, doi:https://doi.org/10.1016/j.jbtep.2013.10.008 (2014).

Chamberlain, S. R., Fineberg, N. A., Blackwell, A. D., Robbins, T. W. \& Sahakian, B. J. Motor inhibition and cognitive flexibility in obsessive-compulsive disorder and trichotillomania. Am J Psychiatry 163, 1282-1284, doi:10.1176/appi.ajp.163.7.1282 (2006). Morrison, A. S. et al. Empathy for Positive and Negative Emotions in Social Anxiety Disorder. Behaviour research and therapy 87, 232-242, doi:10.1016/j.brat.2016.10.005 (2016). Nietlisbach, G., Maercker, A., Rossler, W. \& Haker, H. Are empathic abilities impaired in posttraumatic stress disorder? Psychol Rep 106, 832-844, doi:10.2466/pr0.106.3.832-844 (2010). Parlar, M. et al. Alterations in empathic responding among women with posttraumatic stress disorder associated with childhood trauma. Brain Behav 4, 381-389, doi:10.1002/brb3.215 (2014). Dos Santos, R. G. et al. Antidepressive, anxiolytic, and antiaddictive effects of ayahuasca, psilocybin and lysergic acid diethylamide (LSD): a systematic review of clinical trials published in the last 25 years. Therapeutic Advances in Psychopharmacology 6, 193-213, doi:10.1177/2045125316638008 (2016).

Todd, A. R., Forstmann, M., Burgmer, P., Brooks, A. W. \& Galinsky, A. D. Anxious and egocentric: how specific emotions influence perspective taking. Journal of experimental psychology. General 144, 374-391, doi:10.1037/xge0000048 (2015). Aggression, Maltreatment \& Trauma 18, 382-402, doi:10.1080/10926770902881489 (2009). Aldao, A., Nolen-Hoeksema, S. \& Schweizer, S. Emotion-regulation strategies across psychopathology: A meta-analytic review. Clinical psychology review 30, 217-237 (2010). Baas, M., De Dreu, C. K. \& Nijstad, B. A. A meta-analysis of 25 years of mood-creativity research: hedonic tone, activation, or regulatory focus? Psychol Bull 134, 779-806, doi:10.1037/a0012815 (2008).

Davis, M. A. Understanding the relationship between mood and creativity: A meta-analysis. Organizational Behavior and Human Decision Processes 108, 25-38, doi:https://doi.org/10.1016/i.obhdp.2008.04.001 (2009). 


\section{Sub-acute effects of naturalistic psilocybin use on pro-social behaviors}

Ashby, F. G., Isen, A. M. \& Turken, A. U. A neuropsychological theory of positive affect and its influence on cognition. Psychological review 106, 529-550 (1999).

Forgeard, M. J. C. \& Eichner, K. V. in The Wiley Blackwell handbook of positive psychological interventions. $137-154$ (Wiley-Blackwell, 2014).

Palgi, S., Klein, E. \& Shamay-Tsoory, S. G. Oxytocin improves compassion toward women among patients with PTSD. Psychoneuroendocrinology 64, 143-149, doi:https://doi.org/10.1016/j.psyneuen.2015.11.008 (2016).

Harman, W. W., McKim, R. H., Mogar, R. E., Fadiman, J. \& Stolaroff, M. J. Psychedelic agents in creative problem-solving: A pilot study. Psychological reports 19, 211-227 (1966).

Sessa, B. Is it time to revisit the role of psychedelic drugs in enhancing human creativity? Journal of Psychopharmacology 22, 821-827 (2008).

Kuypers, K. P. C. et al. Ayahuasca enhances creative divergent thinking while decreasing conventional convergent thinking. Psychopharmacology 233, 3395-3403 (2016).

Kuypers, K. P. C. Out of the box: A psychedelic model to study the creative mind. Medical hypotheses 115, 13-16 (2018). Preller, K. H. et al. The effect of 5-HT2A/1a agonist treatment on social cognition, empathy, and social decision-making. European Psychiatry 30, 22 (2015)

Dolder, P. C., Schmid, Y., Müller, F., Borgwardt, S. \& Liechti, M. E. LSD acutely impairs fear recognition and enhances emotional empathy and sociality. Neuropsychopharmacology 41, 2638 (2016).

Krippner, S. The Hypnotic Trance, the Psychedelic Experience, and the Creative Act. American Journal of Clinical Hypnosis 7, 140-147, doi:10.1080/00029157.1964.10402408 (1964).

Uthaug, M. V. et al. Sub-acute and long-term effects of ayahuasca on affect and cognitive thinking style and their association with ego dissolution. Psychopharmacology, 1-11 (2018).

Griffiths, R. R. et al. Psilocybin produces substantial and sustained decreases in depression and anxiety in patients with life-threatening cancer: A randomized double-blind trial. Journal of Psychopharmacology 30, 1181-1197, doi:10.1177/0269881116675513 (2016).

Johnson, M. W., Garcia-Romeu, A. \& Griffiths, R. R. Long-term follow-up of psilocybin-facilitated smoking cessation. The American journal of drug and alcohol abuse 43, 55-60, doi:10.3109/00952990.2016.1170135 (2017).

Carhart-Harris, R. L. et al. Psilocybin with psychological support for treatment-resistant depression: an open-label feasibility study. The Lancet Psychiatry 3, 619-627 (2016).

Hasler, F., Grimberg, U., Benz, M. A., Huber, T. \& Vollenweider, F. X. Acute psychological and physiological effects of psilocybin in healthy humans: a double-blind, placebo-controlled dose-effect study. Psychopharmacology 172, 145-156, doi:10.1007/s00213-003-1640-6 (2004).

Erowid. Mushroom Tea Preparation, <https://erowid.org/plants/mushrooms/mushrooms_prep2.shtml> (2015).

Laussmann, T. \& Meier-Giebing, S. Forensic analysis of hallucinogenic mushrooms and khat (Catha edulisForsk) using cation-exchange liquid chromatography. Forensic Science International 195, 160-164, doi:https://doi.org/10.1016/j.forsciint.2009.12.013 (2010).

Prochazkova, L. et al. Exploring the effect of microdosing psychedelics on creativity in an open-label natural setting. Psychopharmacology 235, 3401-3413 (2018).

Dziobek, I. et al. Dissociation of cognitive and emotional empathy in adults with Asperger syndrome using the Multifaceted Empathy Test (MET). Journal of autism and developmental disorders 38, 464-473, doi:10.1007/s10803-0070486-x (2008).

Hysek, C. M. et al. MDMA enhances emotional empathy and prosocial behavior. Social Cognitive and Affective Neuroscience 9, 1645-1652, doi:10.1093/scan/nst161 (2014).

Kuypers, K. P. C. et al. No evidence that MDMA-induced enhancement of emotional empathy is related to peripheral oxytocin levels or 5-HT1a receptor activation. PloS one 9, e100719 (2014).

Kuypers, K. P. C., Dolder, P. C., Ramaekers, J. G. \& Liechti, M. E. Multifaceted empathy of healthy volunteers after single doses of MDMA: A pooled sample of placebo-controlled studies. Journal of Psychopharmacology (Oxford, England) 31, 589-598, doi:10.1177/0269881117699617 (2017).

Schmid, Y. et al. Differential effects of MDMA and methylphenidate on social cognition. Journal of psychopharmacology (Oxford, England) 28, 847-856, doi:10.1177/0269881114542454 (2014).

Diener, E., Emmons, R. A., Larsen, R. J. \& Griffin, S. The Satisfaction With Life Scale. J Pers Assess 49, 71-75, doi:10.1207/s15327752jpa4901_13 (1985).

Pavot, W., Diener, E., Colvin, C. R. \& Sandvik, E. Further validation of the Satisfaction with Life Scale: evidence for the cross-method convergence of well-being measures. Journal of personality assessment 57, 149-161, doi:10.1207/s15327752jpa5701_17 (1991).

Pavot, W. \& Diener, E. The Satisfaction with Life Scale (SWL). Measurement Instrument Database for the Social Science, $<$ www.midss.ie $>$ (2013).

Pavot, W. \& Diener, E. in Assessing well-being $101-117$ (Springer, 2009). 


\section{Chapter 5}

Carhart-Harris, R. L. et al. Neural correlates of the psychedelic state as determined by fMRI studies with psilocybin. Proceedings of the National Academy of Sciences 109, 2138-2143, doi:10.1073/pnas.1119598109 (2012).

Valle, M. et al. Inhibition of alpha oscillations through serotonin-2A receptor activation underlies the visual effects of ayahuasca in humans. European neuropsychopharmacology : the journal of the European College of Neuropsychopharmacology 26, 1161-1175, doi:10.1016/j.euroneuro.2016.03.012 (2016).

Forgeard, M. J. C. \& Elstein, J. G. Advancing the clinical science of creativity. Frontiers in Psychology 5, 613, doi:10.3389/fpsyg.2014.00613 (2014).

Frecska, E., Móré, C. E., Vargha, A. \& Luna, L. E. Enhancement of creative expression and entoptic phenomena as aftereffects of repeated ayahuasca ceremonies. Journal of psychoactive drugs 44, 191-199 (2012).

Bouso, J. C., Doblin, R., Farre, M., Alcazar, M. A. \& Gomez-Jarabo, G. MDMA-assisted psychotherapy using low doses in a small sample of women with chronic posttraumatic stress disorder. Journal of psychoactive drugs 40, 225-236, doi:10.1080/02791072.2008.10400637 (2008).

Frecska, E., Bokor, P. \& Winkelman, M. The Therapeutic Potentials of Ayahuasca: Possible Effects against Various Diseases of Civilization. Frontiers in Pharmacology 7, 35, doi:10.3389/fphar.2016.00035 (2016).

Barbosa, P. C., Giglio, J. S. \& Dalgalarrondo, P. Altered states of consciousness and short-term psychological after-effects induced by the first time ritual use of ayahuasca in an urban context in Brazil. Journal of psychoactive drugs 37, 193-201, doi:10.1080/02791072.2005.10399801 (2005).

Garcia-Romeu, A., Griffiths, R. R. \& Johnson, M. W. Psilocybin-occasioned Mystical Experiences in the Treatment of Tobacco Addiction. Current drug abuse reviews 7, 157-164 (2015).

Griffiths, R. et al. Psilocybin occasioned mystical-type experiences: immediate and persisting dose-related effects. Psychopharmacology (Berl) 218, 649-665, doi:10.1007/s00213-011-2358-5 (2011).

Osorio, F. L. et al. Antidepressant effects of a single dose of ayahuasca in patients with recurrent depression: a preliminary report. Revista brasileira de psiquiatria (Sao Paulo, Brazil : 1999) 37, 13-20, doi:10.1590/1516-4446-2014-1496 (2015).

Barbosa, P. C., Cazorla, I. M., Giglio, J. S. \& Strassman, R. A six-month prospective evaluation of personality traits, psychiatric symptoms and quality of life in ayahuasca-naive subjects. Journal of psychoactive drugs 41, 205-212, doi:10.1080/02791072.2009.10400530 (2009).

Grob, C. S. et al. Pilot study of psilocybin treatment for anxiety in patients with advanced-stage cancer. Archives of general psychiatry 68, 71-78, doi:10.1001/archgenpsychiatry.2010.116 (2011).

Thomas, G., Lucas, P., Capler, N. R., Tupper, K. W. \& Martin, G. Ayahuasca-assisted therapy for addiction: results from a preliminary observational study in Canada. Current drug abuse reviews 6, 30-42 (2013).

Lawn, W. et al. Well-being, problematic alcohol consumption and acute subjective drug effects in past-year ayahuasca users: a large, international, self-selecting online survey. Scientific Reports 7, 15201, doi:10.1038/s41598-017-14700-6 (2017).

Bouso, J. C. et al. Personality, psychopathology, life attitudes and neuropsychological performance among ritual users of ayahuasca: a longitudinal study. PLoS ONE 7, e42421 (2012).

Morelli, S. A., Lieberman, M. D. \& Zaki, J. The emerging study of positive empathy. Social and Personality Psychology Compass 9, 57-68 (2015).

Kometer, M. et al. Psilocybin Biases Facial Recognition, Goal-Directed Behavior, and Mood State Toward Positive Relative to Negative Emotions Through Different Serotonergic Subreceptors. Biological psychiatry 72, 898-906, doi:https://doi.org/10.1016/j.biopsych.2012.04.005 (2012).

Derntl, B., Seidel, E. M., Schneider, F. \& Habel, U. How specific are emotional deficits? A comparison of empathic abilities in schizophrenia, bipolar and depressed patients. Schizophrenia research 142, 58-64, doi:https://doi.org/10.1016/j.schres.2012.09.020 (2012).

Thomas, M. R. et al. How Do Distress and Well-being Relate to Medical Student Empathy? A Multicenter Study. Journal of General Internal Medicine 22, 177-183, doi:10.1007/s11606-006-0039-6 (2007).

Gleichgerrcht, E. \& Decety, J. Empathy in Clinical Practice: How Individual Dispositions, Gender, and Experience Moderate Empathic Concern, Burnout, and Emotional Distress in Physicians. PLoS ONE 8, e61526, doi:10.1371/journal.pone.0061526 (2013).

Block-Lerner, J., Adair, C., Plumb, J. C., Rhatigan, D. F. \& Orsillo, S. M. The case for mindfulness-based approaches in the cultivation of empathy: does nonjudgmental, present-moment awareness increase capacity for perspective-taking and empathic concern? Journal of marital and family therapy 33, 501-516, doi:10.1111/j.1752-0606.2007.00034.x (2007).

Baer, R. A., Lykins, E. L. B. \& Peters, J. Mindfulness and self-compassion as predictors of psychological wellbeing in longterm meditators and matched nonmeditators. The journal of positive psychology 7, 230-238, doi:10.1080/17439760.2012.674548 (2012).

Gu, J., Strauss, C., Bond, R. \& Cavanagh, K. How do mindfulness-based cognitive therapy and mindfulness-based stress reduction improve mental health and wellbeing? A systematic review and meta-analysis of mediation studies. Clinical psychology review 37, 1-12, doi:https://doi.org/10.1016/j.cpr.2015.01.006 (2015). 
Bowlin, S. L. \& Baer, R. A. Relationships between mindfulness, self-control, and psychological functioning. Personality and Individual Differences 52, 411-415, doi:https://doi.org/10.1016/j.paid.2011.10.050 (2012).

Soler, J. et al. Exploring the therapeutic potential of Ayahuasca: acute intake increases mindfulness-related capacities. Psychopharmacology 233, 823-829, doi:10.1007/s00213-015-4162-0 (2016).

Sampedro, F. et al. Assessing the Psychedelic "After-Glow" in Ayahuasca Users: Post-Acute Neurometabolic and Functional Connectivity Changes Are Associated with Enhanced Mindfulness Capacities. International Journal of Neuropsychopharmacology 20, 698-711, doi:10.1093/ijnp/pyx036 (2017).

80 Shewan, D., Dalgarno, P. \& Reith, G. Perceived risk and risk reduction among ecstasy users: the role of drug, set, and setting. International Journal of Drug Policy 10, 431-453 (2000).

81 Griffiths, R. R., Richards, W. A., McCann, U. \& Jesse, R. Psilocybin can occasion mystical-type experiences having substantial and sustained personal meaning and spiritual significance. Psychopharmacology (Berl) 187, 268-283; discussion 284-292, doi:10.1007/s00213-006-0457-5 (2006). 


\title{
Chapter 6
}

\section{Brainwaves! Spontaneous and deliberate creative cognition during and after psilocybin exposure}

\author{
Mason, N.L., Kuypers, K.P.C., Reckweg, J., Muller, F., Tse, D.H.Y., Da Rios, \\ B., Toennes, S.W., Stiers, P., Feilding, A., Ramaekers, J.G.
}

Creativity is an essential cognitive ability linked to all areas of our everyday functioning. Thus, finding a way to enhance it is of broad interest. A large number of anecdotal reports suggest that the consumption of psychedelic drugs can enhance creative thinking, however scientific evidence is lacking. Following a double-blind, placebo-controlled, parallel-group design, we demonstrated that psilocybin $(0.17 \mathrm{mg} / \mathrm{kg})$ induced a time- and construct-related differentiation of effects on creative thinking. Acutely, psilocybin increased ratings of (spontaneous) creative insights, while decreasing (deliberate) task-based creativity. Seven days after psilocybin, number of novel ideas increased. Furthermore, we utilized an ultra-high field multimodal brain imaging approach and found that acute and persisting effects were predicted by within- and between-network connectivity of the default mode network. Findings add support to historical claims that psychedelics can facilitate aspects of the creative process, making them a potentially novel tool to investigate creativity and subsequent underlying neural mechanisms.

In submission, Translational Psychiatry 


\section{Introduction}

Creativity is an essential cognitive ability linked to all areas of our everyday life, allowing us to adapt to an ever-changing environment and come up with ways to solve problems. Although arguably difficult to define, the creative process has been viewed as a dynamic process ${ }^{1,2}$, requiring shifting between different modes of thought in order to reach an end result ${ }^{3}$. These modes include divergent thinking (DT), which consists of generating novel and original ideas, and convergent thinking (CT), the subsequent evaluation of generated ideas in regards to their usefulness and effectiveness ${ }^{4,5}$. Importantly, as well as being an essential process for every-day functioning, the (in)ability to think "outside of the box" has also been associated with psychological disorders such as depression and anxiety ${ }^{6,7}$. Here it has been regarded both as a transdiagnostic process, i.e. a risk or maintaining factor shared across disorders, and as a potential therapeutic intervention, promoting adaptive interpretations and coping strategies ${ }^{7,8}$. Thus taken together, finding a way to enhance creative thinking is of broad interest amongst multiple disciplines, ranging from industry to psychopathology.

Over the years, a number of anecdotal reports have accumulated suggesting that the consumption of serotonin (5-HT) 2A agonist psychedelic drugs ${ }^{9}$, like lysergic acid diethylamide (LSD), psilocybin, and mescaline, can enhance creativity ${ }^{10,11}$. Famous examples of psychedelicaffiliated creative breakthroughs span the fields of science, technology, and art; including Kary Mullis' discovery of the polymerase chain reaction, the 1960's California based computer industry, and the literary works of authors such as Aldous Huxley and Ken Kesey ${ }^{10,11}$. That said, although there has been much historical interest in the ability of psychedelics to enhance creative capacity, the scientific literature is largely lacking. Initial scientific studies into the effects of psychedelics on creativity date as far back as the 1950's, and ran until the drugs were banned in the United States in the late $1960 \mathrm{~s}^{10,11}$. However, findings of these studies were largely inconclusive, and limited in regards to present-day methodology ${ }^{12,13-14}$. Instead, preliminary contemporary work provides stronger indications that psychedelics can affect creativity-related constructs ${ }^{1,15-19}$, with phenomenological reports suggesting psychedelics induce a hyper-associative state of cognition ${ }^{1}$, and pseudo-experimental studies finding acute ${ }^{20}$ and sub-acute ${ }^{21-23}$ increases in DT or CT after consumption of a psychedelic in a naturalistic setting. Overall, evidence suggests that psychedelics can mediate changes in particular constructs of creativity, however direct, experimental evidence is lacking.

Importantly, if psychedelics can mediate changes in particular dimensions of the creative process, it would make them a potentially novel tool to investigate these processes ${ }^{1}$, as well as the underlying neural mechanisms ${ }^{24}$. In regards to creative cognition, previous research has consistently implicated the coordination of three resting state networks (RSNs); the default mode 
network (DMN), suggested to support idea generation, the frontoparietal control network (FPN), supporting idea evaluation, and areas of the salience network (SN), suggested to facilitate the shift between the internally (DMN) and externally (FPN) oriented cognitive networks ${ }^{25-27}$. Although usually opposing neural processes, the DMN and FPN have been found to interact during creative cognition, suggesting a dynamic shift between these networks facilitates the shift between idea generation and evaluation across time ${ }^{25}$. Recently, this shift between neurocognitive dissociable states has been further conceptualized into a new theory (the dynamic framework of thought ${ }^{1,28}$ ), suggesting that creative thought is an alternation between states with low (DMN driven) to high (FPN driven) cognitive constraints, operating on mnemonic information from the medial temporal region. Interestingly, the authors incorporated the psychedelic state into this model ${ }^{1}$, suggesting that psychedelics represent a state of unconstrained cognition, with neural evidence stemming from previous imaging studies which suggest they acutely induce a flexible brain state ${ }^{29,30} 31$, characterized by an acute disintegration of normally highly organized activity within RSNs $^{32-36}$, a simultaneous widening of dynamic repertoires of connectivity states ${ }^{37}$, and increased coupling of RSNs that are usually anti-correlated ${ }^{34,35,38-40}$. Changes in connectivity have also been found to outlast the acute stage, with reports of increased within-network DMN connectivity 1 day post ${ }^{41-}$ ${ }^{43}$, and increased between network connectivity up to 1 month post drug administration ${ }^{44,45}$.

Overall, previous research suggests an acute and even persisting effect of psychedelics on creativity-related cognition and neural mechanisms. However, no study has yet directly assessed the (sub)acute effect of a psychedelic on the two constructs of creativity (DT and CT) in a controlled trial, or its relationship to drug-induced brain changes. Therefore, the aim of this study was twofold: to assess the acute and persisting (7-day post) effect of the classic psychedelic, psilocybin, on divergent and convergent thinking; and to assess (acute) biological predictors of changes in these constructs. To do this, divergent and convergent thinking were quantified via two widely used cognitive tasks, the Alternate Uses Test (AUT) $)^{46}$ and the Picture Concept Test $(P C T)^{47}$. As evidence suggests a neurocognitive distinction between deliberate (task-based) creative cognition and spontaneous (insight) creative cognition ${ }^{48,49}$, subjective feelings of spontaneous creative thinking were also assessed, via a well-established altered states of consciousness questionnaire ${ }^{50}$. Then, the relationship between cognitive and subjective ratings of creativity, within- and betweennetwork connectivity of the DMN, FPN, and SN, and glutamate concentrations in the medial prefrontal cortex (mPFC) and hippocampus, were evaluated. The latter was of interest as evidence suggests that network connectivity changes during the psychedelic state may result from changes in local network integrity due to downstream stimulation of glutamatergic signaling 51,5243 , this is of particular interest in the MPFC and hippocampus due to their involvement in the FPN and DMN, and their recruitment in creative cognition ${ }^{53-55}$. 
Resting state functional magnetic resonance imaging (rsfMRI) was used to assess RSN functional connectivity (FC), and ultra-high field (7T) proton Magnetic Resonance Spectroscopy (MRS) was used to assess glutamate concentrations in designated brain areas. Overall it was hypothesized that psilocybin would have an acute and persisting increase on outcome measures of DT, which would be predicted by within-network FC of the DMN. It was further hypothesized that psilocybin would decrease CT acutely ${ }^{47}$, which would be predicted by between network FC of the DMN and FPN.

\section{Materials and Methods}

A detailed description of the experimental procedure is provided in the Supplementary Methods and briefly summarized here.

The present study was conducted between July 2017 and June 2018 at Maastricht University, employing a balanced randomized (1:1), placebo-controlled, double-blind, parallel group design. Sixty healthy participants, with previous experience with a psychedelic drug but not within the past 3 months, were allocated to a treatment condition $(0.17 \mathrm{mg} / \mathrm{kg}$ psilocybin or placebo, p.o.). Groups were matched for age, sex, and education level. Full demographic information on the sample is available in Mason, et al. ${ }^{52}$.

Participants visited the lab on 3 separate occasions. The first visit included a familiarization with testing day procedures and completion of the creativity tasks (baseline). The second visit consisted of the formal testing day, with treatment administration (psilocybin or placebo), fMRI, and completion of the creativity tasks (acute). The third testing day took place 7 days after the acute testing day, and included the final completion of the creativity tasks (follow-up).

This study was conducted according to the code of ethics on human experimentation established by the declaration of Helsinki (1964) and amended in Fortaleza (Brazil, October 2013) and in accordance with the Medical Research Involving Human Subjects Act (WMO) and was approved by the Academic Hospital and University's Medical Ethics committee. All participants were fully informed of all procedures, possible adverse reactions, legal rights and responsibilities, expected benefits, and their right for voluntary termination without consequences. The present data were part of a larger clinical trial (Netherlands Trial Register: NTR6505) of which parts have been previously published ${ }^{52}$.

\section{Creativity Tasks}

Parallel (different) versions of each task were used on the baseline, acute, and follow-up testing days. All responses were scored afterwards by two independent raters who were blind to the treatment condition. The interrater reliability was calculated before averaging the ratings. 
(Sub) acute effects of psilocybin on creativity

\section{Picture Concept Task}

The PCT was administered during peak drug effects (about 120 minutes post treatment), as an assessment of both convergent and divergent thinking, and in accordance with previous studies ${ }^{20}$ 23,47. In brief, the PCT consists of 17 stimuli, each containing between 4 and 12 color pictures shown in a matrix. Participants were given 30 seconds per stimulus to find an association between one of the pictures in each row. Specifically, they were asked first to provide the correct solution, as there is only one correct answer. The number of correct answers served as the dependent measure of convergent thinking. In order to assess divergent thinking, participants were then asked to provide as many alternative answers as possible, which are used to calculate several parameters -i.e., fluency, originality, and the ratio of both. Fluency is defined as the number of alternative associations; originality is calculated by evaluating the originality of the alternative association relative to those provided by all other participants in a session; the ratio score is then calculated by originality/fluency.

\section{Alternative Uses Task}

The AUT was administered in accordance with previous publications, at about 130 minutes post treatment, as an assessment of divergent thinking ${ }^{46}$. Participants were asked to list as many possible uses for two common household items and given three minutes per item to do so. Dependent outcome variables were the same as with the PCT, with an addition of the dimension "novelty". Novelty was assessed in order to separate newly generated ideas from recollection of old ideas from memory, and was done so by asking participants after idea generation to indicate if they had given any responses that were completely new to them (i.e. they had never thought of this use before, had never seen it in a movie) $)^{56}$. The number of new responses were then summed up. Additional outcome measures of the AUT that have been assessed in other studies ${ }^{57}$, but were out of the scope of this study, can be found in SI Results.

\section{Subjective state}

The 5 Dimensions of Altered States of Consciousness (5D-ASC) scale ${ }^{50}$ was administered 360 minutes after drug administration, as a retrospective measure of drug effects. Of particular interest for this study was the subscale "insightfulness", consisting of 3 questions conceptually related to spontaneous creative thinking: "I felt very profound", "I had insights into connections that had previously puzzled me", "I had very original thoughts".

\section{Image acquisition}

Participants underwent structural MRI (50 minutes post treatment), single-voxel proton MRS in the mPFC (65 minutes post) and hippocampus (95 minutes post), and rsfMRI (102 minutes post), 
during peak subjective drug effects. Images were acquired on a MAGNETOM 7T MR scanner. At rest, 258 whole-brain EPI volumes were acquired (TR=1400 ms; TE= 21 ms; field of view (FOV)=198 $\mathrm{mm}$; flip angle $=60^{\circ}$; oblique acquisition orientation; interleaved slice acquisition; 72 slices; slice thickness $=1.5 \mathrm{~mm}$; voxel size $=1.5 \times 1.5 \times 1.5 \mathrm{~mm}$ ). During scanning, participants were shown a black cross on a white background, and instructed to look at the cross while attempting to clear their mind, and lay as still as possible.

Spectroscopic voxels were placed by a trained operator at the mPFC (voxel size $=25 \times 20 x$ $17 \mathrm{~mm}^{3}$ ) and the right hippocampus (voxel size $=37 \times 15 \times 15 \mathrm{~mm}^{3}$ ). Spectra were acquired with the stimulated echo acquisition mode $(\mathrm{STEAM})^{58}$ sequence (TE $=6.0 \mathrm{~ms}$, TR $=5.0 \mathrm{~s}, 64$ averages). Outcome measures for MRS were concentration ratios of glutamate to total creatine ( $\mathrm{tCr}$, Creatine + Phospho-Creatine).

Detailed information regarding image acquisition and MRS quantification is previously published $^{52}$.

\section{Processing of imaging data}

Resting state data was processed and analysed using the CONN toolbox $18 . b^{59}$. All volumes were realigned, unwarped, segmented into grey and white matter and cerebrospinal fluid, normalised into a standard stereotactic space (Montreal Neurological Institute) and smoothed with a $6 \mathrm{~mm}$ full width at half maximum Gaussian kernel. Independent component analysis (ICA) was performed using group-ICA procedures implemented in the CONN toolbox following previously described methods ${ }^{60}$. Independent components were restricted to 20 in order to allow comparisons with established RSNs ${ }^{61}$ and previous studies on psilocybin ${ }^{62}$ and LSD $^{34,35}$. Detailed information on the ICA analysis is described in Mason, et al. ${ }^{52}$.

Spectroscopy data, to assess glutamate concentrations in the MPFC and the hippocampus, was analysed with LCModel version 6.3-1H, as described in Mason, et al. ${ }^{52}$.

\section{Pharmacokinetic measures}

Venous blood samples were collected at regular time points after treatment administration (80, 150, and 360 minutes) in order to assess blood serum concentrations of psilocin, the main metabolite of psilocybin.

\section{Statistical analysis}

Statistical analysis of interrater reliability, outcome variables of the creativity tasks, subjective effects, and relative glutamate concentrations were conducted in IBM SPSS Statistics 24 . To assess the interrater reliability, Intra-class Correlation Coefficients (ICC) and their 95\% confidence 
intervals were calculated based on a mean-rating $(k=2)$, consistency, 2-way random-effects model for relevant PCT and AUT dependent variables. Reliability derived from ICC values can range from poor $(<0.5)$, to moderate (0.5-0.75), good (0.75-0.9) and excellent $(>0.90)^{63}$.

Baseline-change scores of the AUT and PCT outcome measures entered a mixed model ANOVA consisting of the between-subject factor Treatment (psilocybin or placebo) and the withinsubject factors Time (acute and follow-up), and Construct (the dependent outcome variables of the PCT and AUT). In case of a Treatment*Time*Construct interaction, independent samples $t$ tests were conducted in order to assess differences between groups. Mann-Whitney $U$ tests were used to assess Treatment group differences in ratings of the 5D-ASC subscale "insightfulness", and differences in relative glutamate concentrations. The alpha criterion level of significance of all aforementioned tests was set at $\mathrm{p}=0.05$ (two-tailed).

For the assessment of within-network FC, the unthresholded ICA component images were compared between placebo and drug conditions (two sample $t$-test). Parametric statistics were used (voxel threshold $p<0.001$ uncorrected, cluster threshold $p<0.05$ cluster-size, false discovery rate (FDR) corrected, two-sided).

For the assessment of between-network FC, ICA components were thresholded at $z>2$ and binarized. The mean of the time series was extracted for every resulting ROI. Time courses between all RSNs were then compared for both conditions using bivariate correlations. The resulting correlation coefficients were compared between placebo and drug conditions (two sample t-test).

Canonical correlations ${ }^{64}$ were conducted to evaluate the association between changes in variables that showed a significant treatment effect, including (i) outcome measures of the PCT and AUT, (ii) ratings of the 5D-ASC dimension, insightfulness, (iii) within- and between-network resting state $\mathrm{FC}$, using extracted connectivity strength (beta) values, and (iv) relative glutamate concentration levels in the mPFC and hippocampus. Variables were separated into two sets; set 1 included the cognitive and subjective measures of creativity [(i) and (ii)] and set 2 included biological variables as predictors [(iii) and (iv)]. An iterative imputation approach was performed to fill in missing data points, when applicable.

\section{Results}

Demographic variables, psilocin blood serum concentrations, subjective state, and relative glutamate concentrations 
Demographic information, mean (S.E.) psilocin blood serum concentrations, ratings on the 5DASC, and relative glutamate concentrations are all previously published elsewhere ${ }^{52}$ and briefly summarized here.

The psilocybin group $(n=30)$ and the placebo group $(n=30)$ did not differ in respect to demographic variables such as gender, age, and drug use history. Psilocin blood serum concentrations reached a peak at 80 minutes post administration $(15.61 \pm 1.66 \mathrm{ng} / \mathrm{mL})$ while participants were in the MRI scanner, and were $12.86 \pm 1.13 \mathrm{ng} / \mathrm{mL}$ during creativity task completion (150 minutes post). Administration of psilocybin was also associated with increased ratings on all (sub)dimensions of the 5D-ASC including the dimension of interest for this study, "insightfulness" ( $n=60, U=44.5 ; p \leq 0.001, d=1.78$ ). Finally, compared to placebo, relative glutamate concentrations were significantly higher in the mPFC (mean \pm S.E.; psilocybin: $1.23 \pm$ $0.02, n=24$; placebo: $1.14 \pm 0.02, n=28 ; U=200.50, p=0.01, d=0.80)$ and significantly lower in the hippocampus (psilocybin: $0.77 \pm 0.03, n=21$; placebo: $0.88 \pm 0.03, n=25 ; U=163.50, p=0.03$, $d=0.69)$.

\section{Creativity Tasks}

The ICC for originality was .95 on the PCT and .91 on the AUT, indicating excellent reliability. The ICC for all outcome variables, including those not within the scope of this study can be found in Table S1.

\section{Picture Concept Test}

Psilocybin influenced performance on the PCT in a time- and construct- dependent manner, as evidenced by a significant Treatment $x$ Time $x$ Construct interaction $\left(F_{3,47}=4.53, p=0.007\right.$; psilocybin $n=25$, placebo $n=26)$. Compared to placebo, psilocybin acutely decreased $C T(d=0.85)$, and measures of DT including fluency $(d=0.84)$, and originality $(d=0.65)$. At the 7 -day follow-up, CT was still significantly decreased when comparing psilocybin to placebo $(d=0.60)$ (Table 1 ; Figure $1)$. 
Table 1. Mean(SE) and independent t-test results (psilocybin vs placebo) of all dependent outcome variables on the AUT and PCT. *statistical significance at the $p=.05$ level.

\begin{tabular}{|c|c|c|c|c|c|c|c|c|c|}
\hline \multicolumn{2}{|c|}{ Variable } & \multicolumn{4}{|l|}{ Acute } & \multicolumn{4}{|l|}{ Longterm } \\
\hline & & Psilocybin & Placebo & $\begin{array}{l}\text { T } \\
\text { value }\end{array}$ & $\begin{array}{l}\mathrm{P} \\
\text { value }\end{array}$ & Psilocybin & Placebo & $\begin{array}{l}\mathrm{T} \\
\text { value }\end{array}$ & $\begin{array}{l}P \\
\text { value }\end{array}$ \\
\hline \multirow[b]{4}{*}{$\frac{5}{2}$} & Fluency & $\begin{array}{l}-6.55 \\
(1.13)\end{array}$ & $\begin{array}{l}-1.89 \\
(1.02)\end{array}$ & -3.06 & $\begin{array}{l}< \\
.01^{*}\end{array}$ & $\begin{array}{l}-2.44 \\
(0.89)\end{array}$ & $\begin{array}{l}-3.62 \\
(0.96)\end{array}$ & 0.89 & .37 \\
\hline & Originality & $\begin{array}{l}-1.62 \\
(0.97)\end{array}$ & $\begin{array}{l}-0.48 \\
(1.22)\end{array}$ & -0.73 & .47 & $\begin{array}{l}-0.14 \\
(0.83)\end{array}$ & $\begin{array}{l}-0.72 \\
(0.77)\end{array}$ & 0.51 & .61 \\
\hline & Ratio & $\begin{array}{l}0.07 \\
(0.03)\end{array}$ & $\begin{array}{l}0.02 \\
(0.05)\end{array}$ & 0.81 & .42 & $\begin{array}{l}0.04 \\
(0.03)\end{array}$ & $\begin{array}{l}0.05 \\
(0.04)\end{array}$ & -0.34 & .73 \\
\hline & Novel & $\begin{array}{l}-0.31 \\
(0.76)\end{array}$ & $\begin{array}{l}-0.21 \\
(0.49)\end{array}$ & -0.11 & .91 & $\begin{array}{l}2.25 \\
(0.76)\end{array}$ & $\begin{array}{l}0.45 \\
(0.53)\end{array}$ & 1.96 & $.05^{*}$ \\
\hline & Fluency & $\begin{array}{l}-2.73 \\
(1.81)\end{array}$ & $\begin{array}{l}4.19 \\
(1.40)\end{array}$ & -3.02 & $\begin{array}{l}< \\
.01^{*}\end{array}$ & $\begin{array}{l}4.07 \\
(1.24)\end{array}$ & $\begin{array}{l}5.25 \\
(1.39)\end{array}$ & -0.63 & .53 \\
\hline & Originality & $\begin{array}{l}-.711 \\
(1.65)\end{array}$ & $\begin{array}{l}4.54 \\
(1.49)\end{array}$ & -2.36 & $.02 *$ & $\begin{array}{l}1.59 \\
(1.32)\end{array}$ & $\begin{array}{l}5.31 \\
(1.52)\end{array}$ & -1.84 & .07 \\
\hline & Ratio & $\begin{array}{l}0.10 \\
(.035)\end{array}$ & $\begin{array}{l}0.09 \\
(.063)\end{array}$ & 0.04 & .96 & $-.04(.03)$ & $\begin{array}{l}.06 \\
(.06)\end{array}$ & -1.53 & .13 \\
\hline Ł & Convergent & $\begin{array}{l}-1.00 \\
(.76)\end{array}$ & $\begin{array}{l}1.61 \\
(.37)\end{array}$ & -3.15 & $\begin{array}{l}< \\
.01^{*}\end{array}$ & $1.67(.57)$ & $\begin{array}{l}3.36 \\
(.49)\end{array}$ & -2.24 & $.03^{*}$ \\
\hline
\end{tabular}

\section{Alternative Uses Test}

Psilocybin influenced performance on the AUT in a time and construct- dependent manner, as evidenced by a significant Treatment $x$ Time $x$ Construct interaction ( $F_{353}=4.50, P=0.007$; psilocybin $\mathrm{n}=28$, placebo $\mathrm{n}=29)$. Compared to placebo, psilocybin acutely decreased fluency $(d=0.80)$. Conversely, compared to placebo psilocybin significantly increased scores of novelty $(d=0.52)$ at the follow-up (Table 1; Figure 1). 


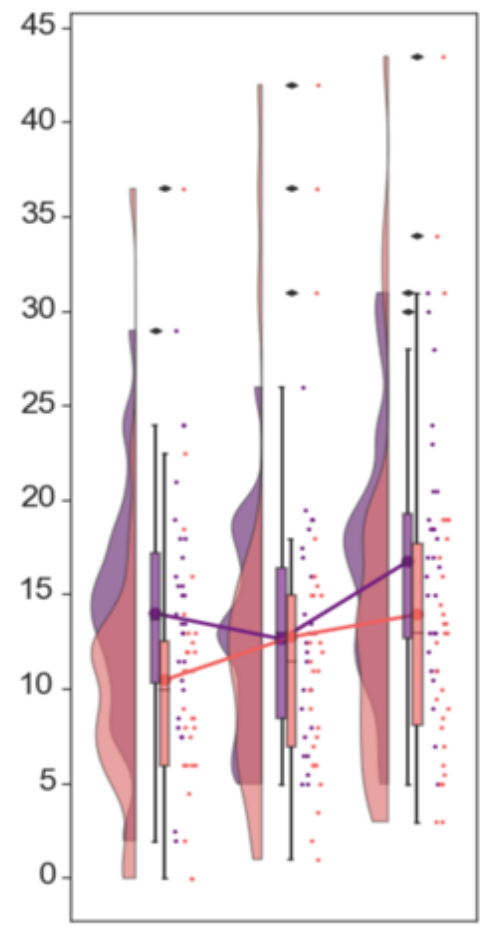

Baseline Acute Follow-up

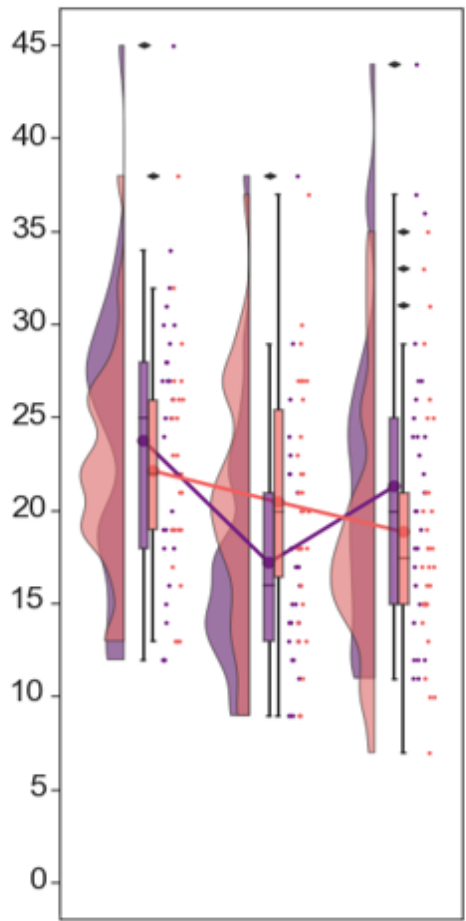

Baseline Acute Follow-up

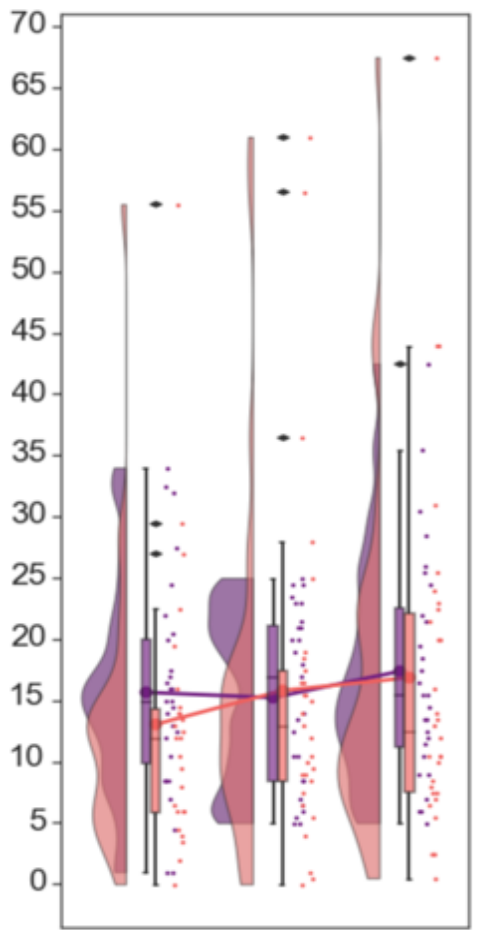

Baseline Acute Follow-up

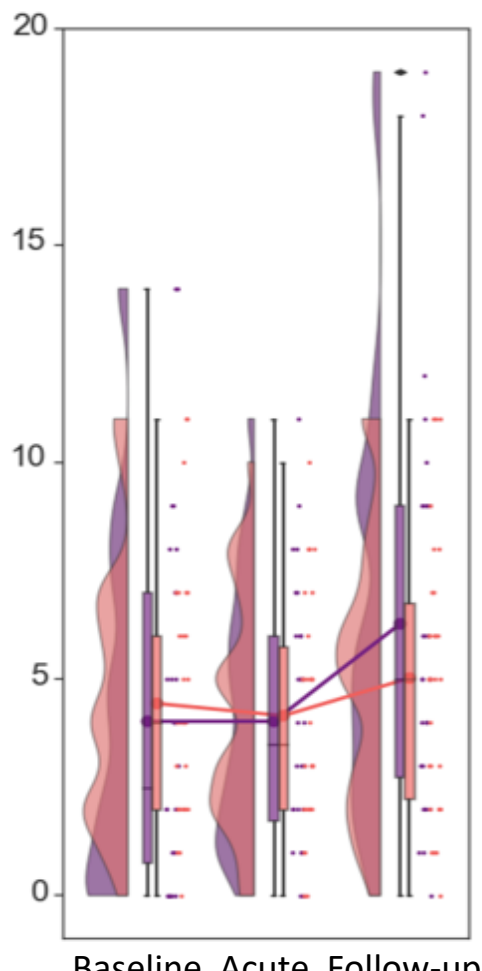

Baseline Acute Follow-up

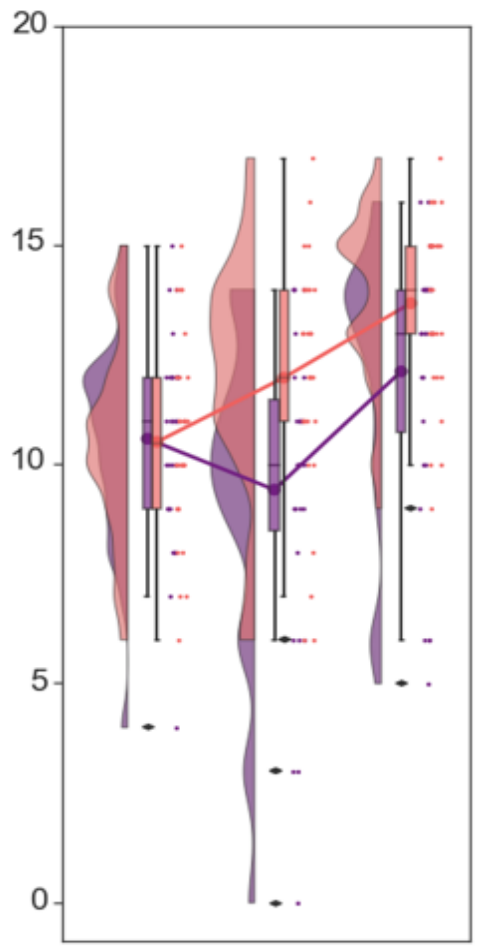

Baseline Acute Follow-up

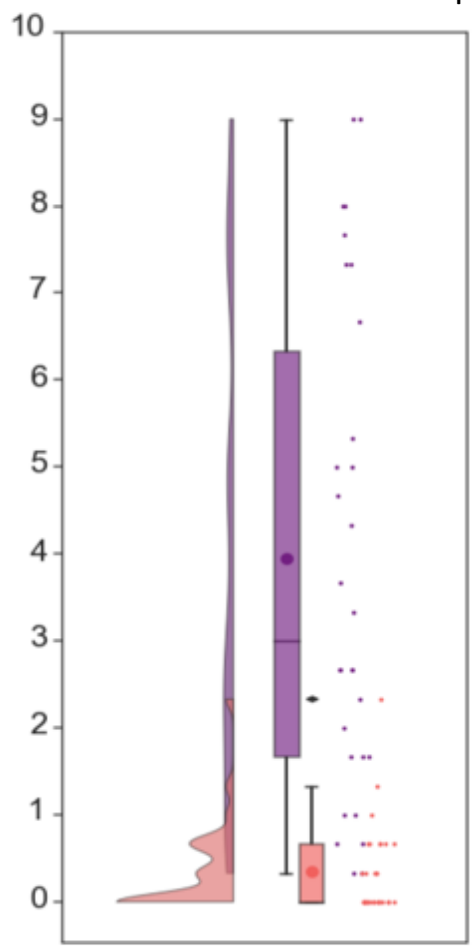

Acute

Psilocybin $\square$ Placebo

Figure 1. Raincloud plots displaying scores on measures of creativity, which demonstrated significant differences between treatment groups. The plot consists of a probability density plot, a boxplot, and raw data points. In the boxplot, the line dividing the box represents the median of the data, the ends represent the upper/lower quartiles, and the extreme lines represent the highest and lowest values excluding outliers. The code for raincloud plot visualization has been adapted from Allen et al. 


\section{Resting state networks}

After quality control of the imaging data, the final sample consisted of 22 participants in the psilocybin group and 26 in the placebo group. There were no significant differences between groups in regards to head motion parameters. See supplementary material for exclusion criteria and assessed differences between groups.

Independent component analysis. There was a good agreement between the components identified in our analysis and the templates provided by Smith, et al. ${ }^{61}$. The results of this full analysis are published elsewhere ${ }^{52}$. For the purpose of this study, we focused on the frontoparietal network 1 ( $r=0.50)$ and $2(r=.47)$ and the DMN, which was split into subcomponents (anterior: $r=0.34$ and posterior: $r=0.52$ ), as has already been observed in multiple studies ${ }^{65,66}$. The anterior salience network ( $r=.39$ ) was identified via the component of Shirer, et al. ${ }^{67}$ as an SN template is not identified in ${ }^{61}$.

Within-network connectivity. Within the respective network, significantly less coactivation under the drug condition relative to placebo was found in both subcomponents of the DMN (anterior and posterior). FC within the FPN and SN did not differ between psilocybin and placebo (Figure 2). 


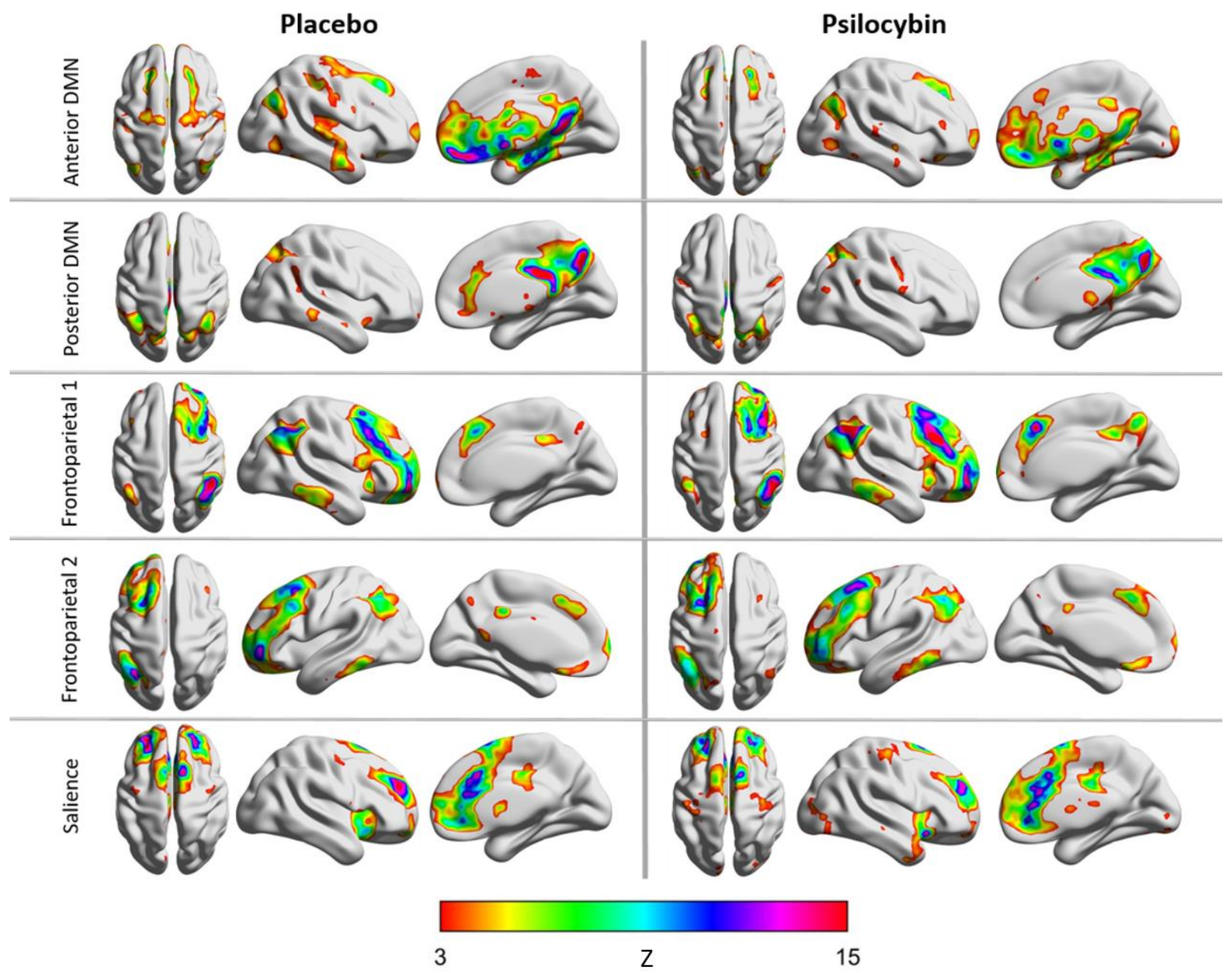

Figure 2. Resting state networks implicated in creative cognition, for each group (placebo and psilocybin). The anterior and posterior DMN demonstrated significant differences in within-network functional connectivity between groups.

Between-network connectivity. Widespread increases in between-network FC were observed under psilocybin compared to placebo, with significant increases between the anterior and posterior DMN and the FPN1, the anterior DMN and the FPN2, and between the anterior DMN and the SN (Figure 3). 


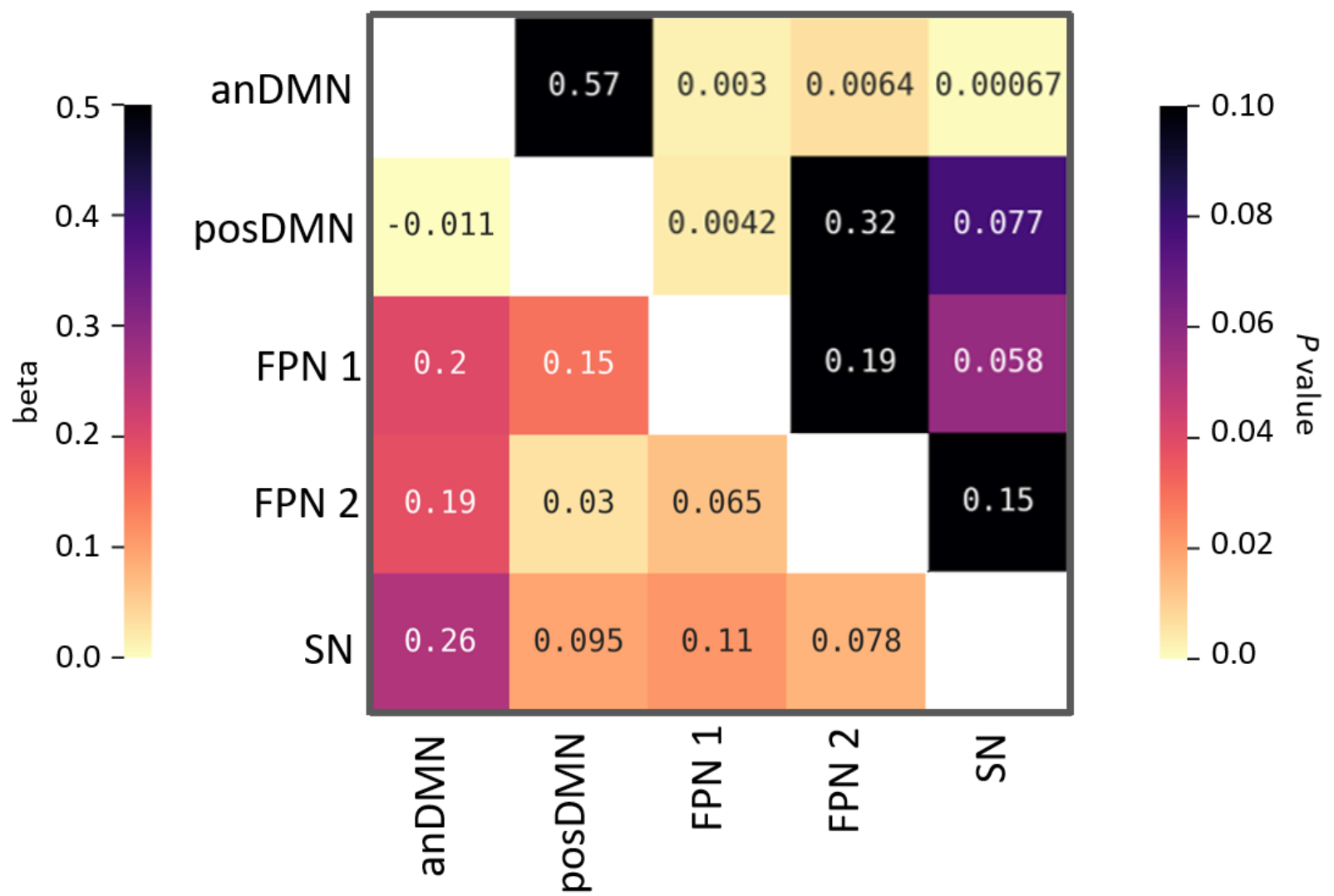

Figure 3. Correlation matrix illustrating increased functional connectivity between resting state networks under psilocybin compared to placebo (psilocybin > placebo). The left half of the matrix refers to the beta values, and the right half of the matrix refers to the $p$ value.

\section{Assessing biological predictors of creativity}

A canonical correlation analysis was conducted using the eight biological variables as predictors of the eight creativity outcome measures to evaluate the multivariate shared relationship between the two variable sets. The analysis yielded seven functions with squared canonical correlations $\left(R_{c}{ }^{2}\right)$ of .553,.483,.165, .135, .075. 042, and .001 for each successive function. The first three functions were considered noteworthy in the context of this study, explaining $55.3 \%, 48.3 \%$, and $16.5 \%$ of the variance, respectively. The full model across all functions was statistically significant $F(56,193.79)=1.48, p=.027)$, explaining $85.3 \%$ of the variance.

Table 2 presents the standardized canonical function coefficients, the structure coefficients $\left(r_{s}\right)$, and the squared structure coefficients $\left(r_{s}{ }^{2}\right)$ for each function as well as the communalities $\left(h^{2}\right)$ across the functions for each variable. Function 1 indicated that the dominant contributor was ratings of insight and the secondary contributor was long-term changes in novel 
responses, with posterior DMN and anterior DMN FC being the dominant predictors, and secondary predictors being hippocampal glutamate levels. Overall, this function suggests that the strongest predictor of increasingly higher feelings of insight and (positive) changes in long-term novelty, were lower levels of within-network DMN FC acutely.

Function 2 indicated that the dominant contributors were the acute changes in originality, with the dominant predictor being posterior DMN FC, suggesting that the strongest predictor of larger (negative) changes in acute originality were lower levels of within-network posterior DMN FC.

Function 3 indicated that the dominant contributors were the acute and long-term changes in convergent thinking, whereas the dominant predictors were FC between both components of the DMN and the FPN. This function suggests that the strongest predictor of larger (negative) changes in acute and long-term CT were higher levels of between network FC between the DMN and the FPN. 
Table 2. Canonical solution for biological variables predicting creativity variables for all functions. $r_{s}$ greater than $\mid .45 /$ and $h^{2}$ greater than $45 \%$ are underlined and deemed valuable contributors.

\begin{tabular}{|c|c|c|c|c|c|c|c|c|c|c|}
\hline & Funct & ion 1 & & Functi & ion 2 & & Functi & ion 3 & & \\
\hline Variable & Coef & $r_{s}$ & $r_{s}^{2}(\%)$ & Coef & $r_{s}$ & $r_{s}^{2}(\%)$ & Coef & $r_{s}$ & $r_{s}^{2}(\%)$ & $h^{2}(\%)$ \\
\hline AUT Fluency & 0.10 & -0.40 & 15.68 & -0.14 & -0.04 & 0.12 & 0.08 & -0.21 & 4.54 & 20.34 \\
\hline РCT Fluency & -0.02 & -0.41 & 16.73 & 1.45 & -0.02 & 0.06 & 0.44 & 0.32 & 10.24 & 27.03 \\
\hline РСТ Originality & -0.17 & -0.45 & 20.70 & -1.66 & $\underline{-0.49}$ & 23.72 & -0.33 & 0.22 & 4.80 & $\underline{49.22}$ \\
\hline РСТ Convergent & 0.11 & -0.38 & 14.67 & 0.11 & 0.08 & 0.61 & 0.75 & $\underline{0.78}$ & 61.00 & 76.27 \\
\hline Insight & 0.83 & $\underline{0.89}$ & 79.21 & -0.39 & -0.21 & 4.37 & 0.62 & 0.29 & 8.12 & $\underline{91.70}$ \\
\hline PCT convergent longterm & -0.32 & -0.44 & 19.71 & -0.02 & 0.03 & 0.10 & 0.22 & $\underline{0.58}$ & 33.52 & $\underline{53.33}$ \\
\hline AUT novelty longterm & 0.24 & $\underline{0.47}$ & 22.37 & 0.33 & 0.38 & 14.75 & -0.21 & -0.21 & 4.33 & 41.44 \\
\hline $\mathrm{R}_{\mathrm{c}}{ }^{2}$ & & & 55.3 & & & 48.3 & & & 16.5 & \\
\hline AnDMN & -0.67 & $\underline{-0.80}$ & 63.36 & -0.94 & -0.17 & 3.03 & -0.18 & 0.04 & 0.17 & $\underline{66.56}$ \\
\hline PosDMN & -0.30 & $\underline{-0.72}$ & 52.13 & 0.92 & $\underline{0.56}$ & 30.80 & -0.38 & -0.15 & 2.22 & $\underline{85.15}$ \\
\hline anDMN - SN & 0.10 & 0.34 & 11.70 & 0.13 & -0.20 & 3.88 & -0.38 & $\underline{-0.53}$ & 28.20 & 43.77 \\
\hline anDMN - FPN 1 & 0.25 & 0.28 & 7.90 & -0.39 & -0.36 & 12.74 & -0.40 & $\underline{-0.67}$ & 44.76 & $\underline{65.40}$ \\
\hline posDMN - FPN 1 & 0.07 & 0.24 & 5.86 & 0.12 & 0.15 & 2.16 & -0.42 & $\underline{-0.70}$ & 49.14 & $\underline{57.16}$ \\
\hline anDMN - FPN 2 & -0.53 & 0.11 & 1.10 & -0.42 & -0.35 & 12.53 & -0.14 & $\underline{-0.46}$ & 21.44 & 35.07 \\
\hline Hippocampal glutamate & -0.22 & $\underline{-0.49}$ & 24.30 & 0.18 & 0.21 & 4.28 & -0.10 & -0.30 & 8.94 & 37.53 \\
\hline mPFC glutamate & 0.21 & 0.37 & 13.62 & 0.24 & 0.03 & 0.07 & -0.28 & -0.30 & 9.18 & 22.87 \\
\hline
\end{tabular}

\section{Discussion}

The present study demonstrates the first modern-day attempt to experimentally assess the (sub)acute effects of a psychedelic on divergent and convergent thinking, the two constructs of creative thinking. Findings demonstrate that psilocybin induces a time- and construct-related differentiation of effects on creative thinking; whereas CT and aspects of DT were shown to 
decrease during the psychedelic state, aspects of DT were shown to increase 7 days later compared to placebo. Interestingly, although scores on deliberate, task-driven creative thinking were lower acutely, individuals under psilocybin reported higher subjective ratings on a measure akin to spontaneous creative thinking. Analyses indicated that alterations in creative thinking were also predicted by acute alterations in RSFC. Whereas disintegration of the DMN predicted both higher scores in spontaneous creative thinking and long-term increases in novelty of generated ideas, increased connectivity between the DMN and the FPN predicted decreases in acute and long-term CT.

\section{Acute and persisting effects of psilocybin on divergent thinking}

Contrary to our hypothesis, results indicate that aspects of DT, or the idea generation phase of the creative process, are impaired during the acute psychedelic state. Namely, participants generated less ideas and associations when under psilocybin compared to placebo, as indicated by significantly lower fluency scores on the AUT and the PCT, respectively. Originality scores were also lower after psilocybin on the PCT, however when controlling quality for quantity (how many responses were given) (ratio), there were no significant differences. Thus when assessing how unique generated responses were, there was no significant difference between treatments. That said, individuals under the influence of psilocybin reported significantly higher ratings of "insightfulness", a construct conceptually related to spontaneous creative thinking (e.g. "I had insights into connections that had previously puzzled me", "I had very original thoughts"). Thus, overall, results suggest psilocybin acutely reduces an individual's volume of new ideas and associations, without modulating quality, in response to goal-directed creativity tasks, while increasing feelings of spontaneous creative insights.

In line with our hypothesis, results indicate an increase in aspects of DT after the acute drug effects have worn off. Namely, 7 days after psilocybin administration, participants generated a higher quantity of novel ideas for uses of an everyday object on the AUT compared to placebo. That said, there were no differences in amount of generated ideas or associations in total, or how original responses were. Accordingly, persisting changes in aspects related to divergent thinking have previously been found at various time-points relative to consumption of a range of psychedelic drugs (see Aday, et al. ${ }^{68}$ for a review).

\section{Acute and persisting effects of psilocybin on convergent thinking}

Convergent thinking can be seen as the second phase in the creative process, focused on evaluating possibilities generated during DT, and narrowing them down to a logical solution ${ }^{4}$. In line with previous studies suggesting that psychedelics decrease conventional, logical thinking $16,17,20$. we found an acute decrement in convergent thinking when comparing psilocybin 
to placebo. When comparing the two treatment groups, we also found that the psilocybin group performed significantly worse compared to placebo at follow-up. However, it should be noted that although there is a significant difference when looking between groups, when looking within groups, there is an increase in performance on the CT in the placebo group when comparing baseline to acute and follow-up, and an increase in performance in the psilocybin group when comparing baseline to the follow-up. Thus, although measures were taken to reduce potential learning effects on the PCT, including administering three different versions of the task, and withholding feedback on performance, our data indicate that this could be taking place. The finding that psilocybin acutely blocked this learning effect adds to the literature that psychedelics disrupt associative learning ${ }^{69}$ and impair executive functioning ${ }^{70}$; effects that have been proposed to be mediated via agonistic activation at the $5-\mathrm{HT}_{2 \mathrm{~A}}$ receptor ${ }^{70}$, and potentially due to impaired attentional performance resulting from an inability to ignore task-irrelevant stimuli ${ }^{71,72}$. This suggestion of learning effects also brings into question findings from recent naturalistic studies which found persisting enhancements in CT on the PCT after intake of a psychedelic ${ }^{21-23,73}$, and emphasizes the importance of a placebo-control.

\section{Do psychedelics enhance creative thinking?}

Results of our study suggests that psychedelics do not enhance creative thinking per se, but rather mediate changes in particular constructs of creative thinking, in a time-dependent manner. Interestingly, these constructs could be interpreted in two different ways, either emphasizing a discrepancy between objective vs subjective creative performance, or highlighting the difference between spontaneous vs deliberate creative cognition.

In regards to the former, our data could suggest that psilocybin acutely impairs the idea generation and evaluation phase of creative thinking, while enhancing the feelings of quality of generated ideas (measured via the subjective questionnaire of insightfulness), despite lack of objective evidence. This discrepancy between a subjective sense of enhanced creative thinking, and objective assessments of creativity is a mismatch that is also found in the early psychedelic creativity literature ${ }^{10}$. Increased feelings of insight, changes in affect, and attribution of meaning to previously neutral stimuli are recognized as common acute effects of psychedelic drugs ${ }^{74-79}$, and thus it is plausible that reports of increased creativity during the acute psychedelic state are due to a subjective sense of creativity enhancement that does not match the objective quality ${ }^{1}$. However, one week later this discrepancy dissipated, in that it was objectively found that the idea generation phase is enhanced, with a higher number of novel ideas reported after psilocybin.

An alternative interpretation of our data could arise from the difference between two processing modes of creative cognition, deliberate vs spontaneous ${ }^{49}$. The deliberate processing mode is initiated when one is focused on meeting explicit task demands, such as in a guided, timed 
situation (as is the case with our tasks), and is characterized as being more attention-demanding, searching along structured and rational belief systems in order to reach a goal ${ }^{49}$. In contrast, spontaneous insights tend to occur in a mental state characterized by unrestrained cognition and defocused attention, and tend to be more random, unfiltered, and bizarre ${ }^{49}$; a mental state almost synonymous with the characterization of the psychedelic state ${ }^{80}$. Thus this discrepancy between acute increases in feelings of insight, but decreased number of ideas, could suggest that psychedelics acutely increase the potential for spontaneous creative thought, while decreasing the potential for deliberate creative cognition. As it has been suggested that productive creative cognition may likely be facilitated by a balance between spontaneous insights and controlled deliberate processing ${ }^{48}$, our data suggest that psychedelics disrupt this balance acutely, whereas sub-acutely this balance is restored, with deliberate creative cognition potentially even enhanced, as evidenced by an increased number of novel ideas one week later. A post-hoc correlation analysis also indicates that there may be a relationship between acute increases in spontaneous creativity, and long-term increases in idea generation, as a significant positive correlation was found between the two (See SI results).

\section{Biological predictors of creative thinking}

As we found that psychedelics can mediate changes in particular constructs of creative thinking, it makes them a novel tool to investigate underlying neural mechanisms of the creative process. Thus, canonical correlations were conducted to predict performance on DT and CT from acute biological parameters hypothesized to underlie creative cognition, including within and between network FC of the DMN, FPN, and SN, and glutamate concentrations in the mPFC and the hippocampus.

Decreased integrity of the DMN was found to be the strongest predictor in regards to increased feelings of acute subjective spontaneous creative thinking (insightfulness). In general, this is in line with previous work that has implicated the DMN in subjective effects representing unconstrained cognition during the psychedelic state ${ }^{1,43,78,81}$, as well as the spontaneous processing mode in creative cognition ${ }^{48}$. Changes in DMN activity and integrity have been repeatedly found during the acute psychedelic state $33,34,36,78,82$, and a disruption of coupling between the medial temporal lobes (MTLs) and the $\mathrm{DMN}^{29}$, has been particularly implicated in explaining some of the key subjective effects of the drugs, including feelings of insightfulness ${ }^{78}$, and an altered sense of self and reality ${ }^{81,83}$. Additionally, DMN and MTL activity have both been implicated in dreaming ${ }^{84}$, a state neurophenomenologically similar to the psychedelic state $\mathrm{e}^{1,80,85,86}$ in that both are characterized by experience of bizarre cognitive phenomena, illogical transitions between thoughts, and vivid sensorimotor imagery ${ }^{87}$. Interestingly, dreaming has been suggested to be the most extreme form of the spontaneous processing mode in creative cognition, giving 
rise to insights that are difficult to ascertain during normal waking consciousness ${ }^{49}$. Thus taken together, our data suggest that the psychedelic state may increase the potential for spontaneous creative thought, and does so via disruption of DMN integrity. Additionally, we found that decreased glutamate in the hippocampus was a secondary predictor in increased ratings of insight. As it has been suggested that MTL activity is a crucial driver of the $\mathrm{DMN}^{29}$, and the relationship between the two has been implicated in the psychedelic and related states, it could be suggested that that glutamatergic activity of hippocampal regions could underlie this desynchronization. The involvement of the hippocampus in creative cognition is also interesting to speculate on, as it has been found that past knowledge about a problem can actually be detrimental to solving it, constraining potential ideas to what is already known, and thus is suggested to be a disadvantage of the deliberate mode of creative cognition, and an advantage of the spontaneous mode(see Dietrich ${ }^{49}$ for a review).

In regards to deliberate, task-based creative thinking, the finding that decreased withinnetwork DMN FC predicted both an acute decrease in scores of originality, and a long-term increase in generation of novel ideas may seem counterintuitive, however actually is in line with previous work. Specifically, given the suggested role of the DMN in underlying the idea generation process of DT, an acute decrement in DMN FC resulting in an acute decrement in DT performance on a creativity task would be expected. Additionally, previous work has found that while psychedelics decrease within-network DMN FC acutely, they increase DMN integrity sub-acutely ${ }^{41-}$ 44 , potentially via a neuroplastic effect on brain network function ${ }^{44,88,89}$. Thus, it could be suggested that the latter prediction seen here is reflecting the subsequent psilocybin-induced increase in DMN FC that could facilitate an increase in novel ideas. Such findings add support to the suggestion that novel idea generation is mediated via DMN network $\mathrm{FC}^{1,25-27}$, and also suggest that current frameworks conceptualizing the impact of the psychedelic state on creativity could be adapted to include both acute and persisting effects of the drug ${ }^{1}$.

Finally, levels of acute connectivity between both subcomponents of the DMN and the FPN were found to inversely predict acute and long-term changes in CT performance. Namely, increasingly positive DMN-FPN connectivity predicted increasingly worse CT performance relative to baseline. Although usually anticorrelated ${ }^{90,91}$, previous studies have found that, during a task, higher dynamic DMN-FPN connectivity predicted better performance in areas such as creative cognition, cognitive flexibility, attention, and learning $25,26,92-95$. In regards to creative cognition in particular, it has been suggested that during the creative process, the degree of FPN involvement increases as the need for more deliberate constraints on thought content (in order to reach e.g. a goal) increases ${ }^{1}$. Further, it has been suggested that productive creative cognition is facilitated by a balance between spontaneous and controlled processing ${ }^{49}$, both of which may be experienced concurrently via coupling between the DMN and task positive networks like the FPN ${ }^{48}$. Thus, the 
association between DMN-FPN connectivity and CT is in line with previous work implicating DMNFPN dynamics in the idea evaluation phase of the creative process. That said, it may then be hypothesized that increased DMN-FPN coupling would result in better performance on a task of CT. However, the aforementioned evidence stems from measurements taken while individuals were performing a task. Instead, it has been found that higher resting state FPN-DMN dynamics relates to poorer performance on tasks of cognitive flexibility, and has also been found in states characterized by poorer attention, including during the psychedelic state ${ }^{62}$, and in patients with ADHD ${ }^{96}$. Thus increased DMN-FPN between network connectivity at rest could be reflecting the potential of either intrusion of, or insufficient suppression of, the DMN during a task. As increased resting state DMN-FPN connectivity has also been found in patients characterized by poorer attentional abilities, and psychedelics have been found to disrupt executive functions potentially via impairment of attention ${ }^{71,72}$, this could provide a biological basis for how psychedelics impair executive functioning, and could explain how psilocybin blocked the CT learning effect seen during our study.

Although the role of the mPFC has been strongly highlighted in creative cognition ${ }^{49}$, glutamate concentrations in this area did not play a significant role in the canonical model. This suggests that the involvement of the MPFC is due to its role in integration and interaction of distributed brain areas operating in large-scale networks ${ }^{97}$, rather than underlying neural activity in the specific brain area. Additionally, although the connectivity between the DMN and the SN has been implicated in the creative process, in that the SN has been suggested to facilitate the shift of ideas from the DMN to the FPN ${ }^{25-27}$, we did not find that this strongly predicted any outcome measures of creative thinking. The latter may be due to the fact that we are correlating network activity at rest, with behavior measures taken outside of the scanner, emphasizing a limitation of this study. Thus, although consistent with the previously discussed literature, it is important to note that discrepancies have been found between state and trait network connectivity and creative performance ${ }^{98,99}$. In light of this, results should be taken as preliminary, and future studies assessing the neurobiological effects of psychedelics on creativity should employ creativity tasks during scanning.

\section{Conclusion.}

In conclusion, this study found that psilocybin induces a time- and construct-related differentiation of effects on creative thinking, suggesting that psychedelics are a novel tool to investigate underlying neural mechanisms of the creative process ${ }^{1,24}$. Additionally, these findings add some support to the historical claims that psychedelics can facilitate aspects of the creative process, reducing conventional, logical thinking and giving rise to novel thoughts, but emphasizes the 
distinction between spontaneous and deliberate creative cognition, as well as acute and persisting effects of the drug.

These distinctions are of particular importance as psychedelics are currently being investigated to treat a number of mental health disorders ${ }^{100}$ characterized by rigid, inflexible thought patterns $29,101,102$. Thus, it could be suggested that the ability of psilocybin to acutely decrease CT and increase spontaneous DT, while subacutely enhancing more goal-directed DT, could aid in the therapeutic process by opening up a window of opportunity where therapeutic interventions could prove more effective. Namely, while under the influence of a psychedelic, rigid thought content (CT) could be decreased, while unguided, spontaneous thoughts may give rise to new insights and perspectives of previous events and current problems ${ }^{19,103,104}$. Subacutely, patients may then be able to integrate these insights with a therapist, and come up with new, more effective strategies that facilitate adaptive interpretation and coping abilities ${ }^{8}$. Nevertheless, future studies should be employed in clinical populations in order to assess this proposal, as well as the underlying neurobiological mechanisms.

\section{Funding and disclosure}

This study is part of the Beckley/Maastricht Research Programme. The Beckley Foundation made a financial contribution to the study. The authors report no other relevant funding, and all authors report no potential conflicts of interest.

\section{Acknowledgements}

The authors would like to thank Cees van Leeuwen for medical supervision, Fulya Onus and Sofia Rocha for their assistance with data collection, Safak Erener for assistance with rating responses, Pablo Malloroni for his comments on the manuscript, and all participants for their time and effort.

\section{Author Contributions.}

NM, KK, JG and AF designed the research. NM, and JR performed the research. DT set up the MR/MRS sequences. NM, FM, and BDR analyzed the data. All authors made a substantial contribution to interpretation of the data and drafting of the manuscript. 


\section{Chapter 6}

\section{References}

1 Girn, M., Mills, C., Roseman, L., Carhart-Harris, R. L. \& Christoff, K. Updating the dynamic framework of thought: Creativity and psychedelics. Neurolmage 213, 116726, doi:https://doi.org/10.1016/j.neuroimage.2020.116726 (2020).

2 Runco, M. A. in Dynamic perspectives on creativity 181-188 (Springer, 2019).

3 Guilford, J. P. The nature of human intelligence. (McGraw-Hill, 1967).

4 Runco, M. A. \& Jaeger, G. J. The Standard Definition of Creativity. Creativity Research Journal 24, 92-96, doi:10.1080/10400419.2012.650092 (2012).

5 Cropley, A. In Praise of Convergent Thinking. Creativity Research Journal 18, 391-404, doi:10.1207/s15326934crj1803_13 (2006).

6 Fresco, D. M., Williams, N. L. \& Nugent, N. R. Flexibility and Negative Affect: Examining the Associations of Explanatory Flexibility and Coping Flexibility to Each Other and to Depression and Anxiety. Cognitive Therapy and Research 30, 201210, doi:10.1007/s10608-006-9019-8 (2006).

Forgeard, M. J. C. \& Elstein, J. G. Advancing the clinical science of creativity. Frontiers in Psychology 5, 613, doi:10.3389/fpsyg.2014.00613 (2014).

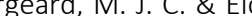
(Wiley-Blackwell, 2014) Nichols, D. E. Psychedelics. Pharmacological reviews 68, 264-355, doi:10.1124/pr.115.011478 (2016). Baggott, M. J. Psychedelics and creativity: a review of the quantitative literature. PeerJ PrePrints 3, e1202v1201, doi:10.7287/peerj.preprints.1202v1 (2015).

Sessa, B. Is it time to revisit the role of psychedelic drugs in enhancing human creativity? Journal of Psychopharmacology 22, 821-827 (2008).

McGlothlin, W., Cohen, S. \& McGlothlin, M. S. Long lasting effects of LSD on normals. Archives of general psychiatry 17 521-532, doi:10.1001/archpsyc.1967.01730290009002 (1967).

Zegans, L. S., Pollard, J. C. \& Brown, D. The effects of LSD-25 on creativity and tolerance to regression. Archives of general psychiatry 16, 740-749, doi:10.1001/archpsyc.1967.01730240096014 (1967).

Harman, W. W., McKim, R. H., Mogar, R. E., Fadiman, J. \& Stolaroff, M. J. Psychedelic agents in creative problem-solving: A pilot study. Psychological reports 19, 211-227 (1966).

Preller, K. H. et al. The fabric of meaning and subjective effects in LSD-induced states depend on serotonin $2 \mathrm{~A}$ receptor activation. Current Biology 27, 451-457 (2017).

Kraehenmann, R. et al. LSD increases primary process thinking via serotonin 2A receptor activation. Frontiers in pharmacology 8, 814 (2017).

Family, N. et al. Semantic activation in LSD: evidence from picture naming. Language, Cognition and Neuroscience 31 , 1320-1327 (2016).

Murphy-Beiner, A. \& Soar, K. Ayahuasca's 'afterglow': improved mindfulness and cognitive flexibility in ayahuasca drinkers. Psychopharmacology (Berl) 237, 1161-1169, doi:10.1007/s00213-019-05445-3 (2020).

Frecska, E., Móré, C. E., Vargha, A. \& Luna, L. E. Enhancement of creative expression and entoptic phenomena as aftereffects of repeated ayahuasca ceremonies. Journal of psychoactive drugs 44, 191-199 (2012). Kuypers, K. P. C. et al. Ayahuasca enhances creative divergent thinking while decreasing conventional convergent thinking. Psychopharmacology (Berl) 233, 3395-3403, doi:10.1007/s00213-016-4377-8 (2016).

Mason, N. L., Mischler, E., Uthaug, M. V. \& Kuypers, K. P. Sub-acute effects of psilocybin on empathy, creative thinking and subjective well-being. Journal of psychoactive drugs 51, 123-134 (2019).

Uthaug, M. V. et al. A single inhalation of vapor from dried toad secretion containing 5-methoxy-N,N-dimethyltryptamine (5-MeO-DMT) in a naturalistic setting is related to sustained enhancement of satisfaction with life, mindfulness-related capacities, and a decrement of psychopathological symptoms. Psychopharmacology (Berl) 236, 2653-2666, doi:10.1007/s00213-019-05236-w (2019).

Uthaug, M. V. et al. Sub-acute and long-term effects of ayahuasca on affect and cognitive thinking style and their association with ego dissolution. Psychopharmacology, 1-11 (2018). Kuypers, K. P. C. Out of the box: A psychedelic model to study the creative mind. Medical hypotheses 115, 13-16 (2018). Beaty, R. E., Benedek, M., Barry Kaufman, S. \& Silvia, P. J. Default and Executive Network Coupling Supports Creative Idea Production. Scientific Reports 5, 10964, doi:10.1038/srep10964 (2015).

Beaty, R. E. et al. Robust prediction of individual creative ability from brain functional connectivity. Proceedings of the National Academy of Sciences 115, 1087-1092, doi:10.1073/pnas.1713532115 (2018).

Beaty, R. E., Benedek, M., Silvia, P. J. \& Schacter, D. L. Creative Cognition and Brain Network Dynamics. Trends in cognitive sciences 20, 87-95, doi:10.1016/j.tics.2015.10.004 (2016).

Christoff, K., Irving, Z. C., Fox, K. C. R., Spreng, R. N. \& Andrews-Hanna, J. R. Mind-wandering as spontaneous thought: a dynamic framework. Nature Reviews Neuroscience 17, 718-731, doi:10.1038/nrn.2016.113 (2016). psychedelic drugs. Front Hum Neurosci 8 (2014). 
Carhart-Harris, R. L. \& Friston, K. J. REBUS and the Anarchic Brain: Toward a Unified Model of the Brain Action of Psychedelics. Pharmacological reviews 71, 316-344, doi:10.1124/pr.118.017160 (2019).

Carhart-Harris, R. L. et al. The paradoxical psychological effects of lysergic acid diethylamide (LSD). Psychological medicine 46, 1379-1390 (2016).

Muthukumaraswamy, S. D. et al. Broadband Cortical Desynchronization Underlies the Human Psychedelic State. The Journal of Neuroscience 33, 15171-15183, doi:10.1523/jneurosci.2063-13.2013 (2013).

Palhano-Fontes, F. et al. The psychedelic state induced by ayahuasca modulates the activity and connectivity of the default mode network. PLOS ONE 10, e0118143 (2015).

Müller, F., Dolder, P. C., Schmidt, A., Liechti, M. E. \& Borgwardt, S. Altered network hub connectivity after acute LSD administration. Neurolmage: Clinical 18, 694-701, doi:https://doi.org/10.1016/j.nicl.2018.03.005 (2018).

Carhart-Harris, R. L. et al. Neural correlates of the LSD experience revealed by multimodal neuroimaging. Proceedings of the National Academy of Sciences of the United States of America 113, 4853-4858, doi:http://dx.doi.org/10.1073/pnas.1518377113 (2016).

Carhart-Harris, R. L. et al. Neural correlates of the psychedelic state as determined by fMRI studies with psilocybin. Proceedings of the National Academy of Sciences 109, 2138-2143, doi:10.1073/pnas.1119598109 (2012).

Tagliazucchi, E., Carhart-Harris, R., Leech, R., Nutt, D. \& Chialvo, D. R. Enhanced repertoire of brain dynamical states during the psychedelic experience. Human brain mapping 35, 5442-5456, doi:10.1002/hbm.22562 (2014).

Carhart-Harris et al. Functional connectivity measures after psilocybin inform a novel hypothesis of early psychosis. Schizophrenia bulletin 39, 1343-1351, doi:10.1093/schbul/sbs117 (2013).

Roseman, L., Leech, R., Feilding, A., Nutt, D. J. \& Carhart-Harris, R. L. The effects of psilocybin and MDMA on betweennetwork resting state functional connectivity in healthy volunteers. Frontiers in Human Neuroscience 8, doi:10.3389/fnhum.2014.00204 (2014).

Tagliazucchi, E. et al. Increased Global Functional Connectivity Correlates with LSD-Induced Ego Dissolution. Current biology : CB 26, 1043-1050, doi:10.1016/j.cub.2016.02.010 (2016).

Sampedro, F. et al. Assessing the Psychedelic "After-Glow" in Ayahuasca Users: Post-Acute Neurometabolic and Functional Connectivity Changes Are Associated with Enhanced Mindfulness Capacities. International Journal of Neuropsychopharmacology 20, 698-711, doi:10.1093/ijnp/pyx036 (2017).

Carhart-Harris, R. L. et al. Psilocybin for treatment-resistant depression: fMRI-measured brain mechanisms. Scientific Reports 7, 13187, doi:10.1038/s41598-017-13282-7 (2017).

Smigielski, L., Scheidegger, M., Kometer, M. \& Vollenweider, F. X. Psilocybin-assisted mindfulness training modulates selfconsciousness and brain default mode network connectivity with lasting effects. Neurolmage 196, 207-215 (2019).

Barrett, F. S., Doss, M. K., Sepeda, N. D., Pekar, J. J. \& Griffiths, R. R. Emotions and brain function are altered up to one month after a single high dose of psilocybin. Scientific Reports 10, 2214, doi:10.1038/s41598-020-59282-y (2020).

Pasquini, L., Palhano-Fontes, F. \& Araujo, D. B. Subacute effects of the psychedelic ayahuasca on the salience and default mode networks. Journal of psychopharmacology (Oxford, England), 269881120909409, doi:10.1177/0269881120909409 (2020).

Kaufman, J. C., Plucker, J. A. \& Baer, J. Essentials of creativity assessment. Vol. 53 (John Wiley \& Sons, 2008).

Kuypers, K. et al. Ayahuasca enhances creative divergent thinking while decreasing conventional convergent thinking. Psychopharmacology 233, 3395-3403 (2016).

Mok, L. W. The interplay between spontaneous and controlled processing in creative cognition. Front Hum Neurosci 8 , 663, doi:10.3389/fnhum.2014.00663 (2014).

Dietrich, A. The cognitive neuroscience of creativity. Psychonomic bulletin \& review 11, 1011-1026 (2004).

Dittrich, A. The standardized psychometric assessment of altered states of consciousness (ASCs) in humans. Pharmacopsychiatry 31, 80-84 (1998).

Aghajanian, G. K. \& Marek, G. J. Serotonin model of schizophrenia: emerging role of glutamate mechanisms. Brain research. Brain research reviews 31, 302-312 (2000).

Mason, N. L. et al. Me, myself, bye: regional alterations in glutamate and the experience of ego dissolution with psilocybin. Neuropsychopharmacology, doi:10.1038/s41386-020-0718-8 (2020).

Fink, A. et al. Enhancing creativity by means of cognitive stimulation: Evidence from an fMRI study. Neurolmage 52, 16871695, doi:https://doi.org/10.1016/j.neuroimage.2010.05.072 (2010).

Beaty, R. E. et al. Default network contributions to episodic and semantic processing during divergent creative thinking: A representational similarity analysis. Neurolmage 209, 116499, doi:https://doi.org/10.1016/j.neuroimage.2019.116499 (2020).

Madore, K. P., Thakral, P. P., Beaty, R. E., Addis, D. R. \& Schacter, D. L. Neural Mechanisms of Episodic Retrieval Support Divergent Creative Thinking. Cerebral Cortex 29, 150-166, doi:10.1093/cercor/bhx312 (2017).

Benedek, M. et al. To create or to recall? Neural mechanisms underlying the generation of creative new ideas. Neurolmage 88, 125-133, doi:https://doi.org/10.1016/j.neuroimage.2013.11.021 (2014). 


\section{Chapter 6}

Silvia, P. J. et al. Assessing creativity with divergent thinking tasks: Exploring the reliability and validity of new subjective scoring methods. Psychology of Aesthetics, Creativity, and the Arts 2, 68 (2008).

Frahm, J. et al. Localized high-resolution proton NMR spectroscopy using stimulated echoes: initial applications to human brain in vivo. Magnetic resonance in medicine 9, 79-93 (1989).

Whitfield-Gabrieli, S. \& Nieto-Castanon, A. Conn: a functional connectivity toolbox for correlated and anticorrelated brain networks. Brain connectivity 2, 125-141, doi:10.1089/brain.2012.0073 (2012).

Calhoun, V. D., Adali, T., Pearlson, G. D. \& Pekar, J. J. A method for making group inferences from functional MRI data using independent component analysis. Human brain mapping 14, 140-151, doi:10.1002/hbm.1048 (2001).

Smith, S. M. et al. Correspondence of the brain's functional architecture during activation and rest. Proceedings of the National Academy of Sciences of the United States of America 106, 13040-13045, doi:10.1073/pnas.0905267106 (2009). Carhart-Harris, R. L. et al. Functional connectivity measures after psilocybin inform a novel hypothesis of early psychosis. Schizophrenia bulletin 39, 1343-1351, doi:http://dx.doi.org/10.1093/schbul/sbs117 (2013).

Koo, T. K. \& Li, M. Y. A Guideline of Selecting and Reporting Intraclass Correlation Coefficients for Reliability Research. Journal of Chiropractic Medicine 15, 155-163, doi:https://doi.org/10.1016/j.jcm.2016.02.012 (2016).

Sherry, A. \& Henson, R. K. Conducting and interpreting canonical correlation analysis in personality research: A userfriendly primer. Journal of personality assessment 84, 37-48 (2005).

Abou-Elseoud, A. et al. The effect of model order selection in group PICA. Human brain mapping 31, 1207-1216, doi:10.1002/hbm.20929 (2010).

Allen, E. A. et al. A baseline for the multivariate comparison of resting-state networks. Frontiers in systems neuroscience 5, 2-2, doi:10.3389/fnsys.2011.00002 (2011).

Shirer, W. R., Ryali, S., Rykhlevskaia, E., Menon, V. \& Greicius, M. D. Decoding subject-driven cognitive states with wholebrain connectivity patterns. Cerebral cortex (New York, N.Y. : 1991) 22, 158-165, doi:10.1093/cercor/bhr099 (2012).

Aday, J. S., Mitzkovitz, C. M., Bloesch, E. K., Davoli, C. C. \& Davis, A. K. Long-term effects of psychedelic drugs: A systematic review. Neuroscience \& Biobehavioral Reviews 113, 179-189, doi:https://doi.org/10.1016/j.neubiorev.2020.03.017 (2020).

Barrett, F. S., Carbonaro, T. M., Hurwitz, E., Johnson, M. W. \& Griffiths, R. R. Double-blind comparison of the two hallucinogens psilocybin and dextromethorphan: effects on cognition. Psychopharmacology 235, 2915-2927, doi:10.1007/s00213-018-4981-x (2018).

Pokorny, T., Duerler, P., Seifritz, E., Vollenweider, F. X. \& Preller, K. H. LSD acutely impairs working memory, executive functions, and cognitive flexibility, but not risk-based decision-making. Psychological medicine, 1-10, doi:10.1017/S0033291719002393 (2019).

Umbricht, D. et al. Effects of the 5-HT 2A agonist psilocybin on mismatch negativity generation and AX-continuous performance task: implications for the neuropharmacology of cognitive deficits in schizophrenia. Neuropsychopharmacology 28, 170-181 (2003).

Carter, O. L. et al. Psilocybin links binocular rivalry switch rate to attention and subjective arousal levels in humans. Psychopharmacology 195, 415-424 (2007).

Prochazkova, L. et al. Exploring the effect of microdosing psychedelics on creativity in an open-label natural setting. Psychopharmacology 235, 3401-3413 (2018).

Griffiths, R. R., Richards, W. A., Johnson, M. W., McCann, U. D. \& Jesse, R. Mystical-type experiences occasioned by psilocybin mediate the attribution of personal meaning and spiritual significance 14 months later. Journal of psychopharmacology 22, 621-632 (2008).

Griffiths, R. R., Richards, W. A., McCann, U. \& Jesse, R. Psilocybin can occasion mystical-type experiences having substantial and sustained personal meaning and spiritual significance. Psychopharmacology (Berl) 187, 268-283; discussion 284-292, doi:10.1007/s00213-006-0457-5 (2006).

Hartogsohn, I. The meaning-enhancing properties of psychedelics and their mediator role in psychedelic therapy, spirituality, and creativity. Frontiers in neuroscience 12, 129 (2018).

Preller, K. H. et al. The role of the serotonin $2 \mathrm{~A}$ receptor in the fabric and modulation of personal meaning in lysergic acid diethylamide (LSD)-induced states. European Neuropsychopharmacology 26, S299-S300 (2016).

Kometer, M., Pokorny, T., Seifritz, E. \& Volleinweider, F. X. Psilocybin-induced spiritual experiences and insightfulness are associated with synchronization of neuronal oscillations. Psychopharmacology 232, 3663-3676, doi:http://dx.doi.org/10.1007/s00213-015-4026-7 (2015).

Studerus, E., Kometer, M., Hasler, F. \& Vollenweider, F. X. Acute, subacute and long-term subjective effects of psilocybin in healthy humans: a pooled analysis of experimental studies. Journal of psychopharmacology (Oxford, England) 25, 14341452, doi:10.1177/0269881110382466 (2011).

Kraehenmann, R. Dreams and psychedelics: neurophenomenological comparison and therapeutic implications. Current neuropharmacology 15, 1032-1042 (2017).

Lebedev, A. V. et al. Finding the self by losing the self: Neural correlates of ego-dissolution under psilocybin. Human brain mapping 36, 3137-3153, doi:10.1002/hbm.22833 (2015). 
Speth, J. et al. Decreased mental time travel to the past correlates with default-mode network disintegration under lysergic acid diethylamide. Journal of psychopharmacology (Oxford, England) 30, 344-353, doi:10.1177/0269881116628430 (2016).

Carhart-Harris, R. L. \& Friston, K. J. The default-mode, ego-functions and free-energy: a neurobiological account of Freudian ideas. Brain : a journal of neurology 133, 1265-1283, doi:10.1093/brain/awq010 (2010). functional neuroimaging and first-person content reports. Frontiers in Human Neuroscience 7, doi:10.3389/fnhum.2013.00412 (2013). Carhart-Harris, R. \& Nutt, D. Was it a vision or a waking dream? Frontiers in Psychology 5, 255 (2014). Sanz, C. \& Tagliazucchi, E. The Experience Elicited by Hallucinogens Presents the Highest Similarity to Dreaming within a Large Database of Psychoactive Substance Reports. Frontiers in neuroscience 12, 7, doi:10.3389/fnins.2018.00007 (2018). Kraehenmann, R. et al. Dreamlike effects of LSD on waking imagery in humans depend on serotonin 2A receptor activation. Psychopharmacology (Berl), doi:10.1007/s00213-017-4610-0 (2017). Vollenweider, F. X. \& Kometer, M. The neurobiology of psychedelic drugs: implications for the treatment of mood disorders. Nature reviews. Neuroscience 11, 642-651, doi:10.1038/nrn2884 (2010)

Ly, C. et al. Psychedelics Promote Structural and Functional Neural Plasticity. Cell reports 23, 3170-3182, doi:10.1016/j.celrep.2018.05.022 (2018).

Cole, M. W., Yarkoni, T., Repovš, G., Anticevic, A. \& Braver, T. S. Global connectivity of prefrontal cortex predicts cognitive control and intelligence. Journal of Neuroscience 32, 8988-8999 (2012). Anticevic, A. et al. The role of default network deactivation in cognition and disease. Trends in cognitive sciences 16, $584-$ 592 (2012).

Douw, L., Wakeman, D. G., Tanaka, N., Liu, H. \& Stufflebeam, S. M. State-dependent variability of dynamic functional connectivity between frontoparietal and default networks relates to cognitive flexibility. Neuroscience 339, 12-21, doi:https://doi.org/10.1016/j.neuroscience.2016.09.034 (2016). Bassett, D. S. et al. Dynamic reconfiguration of human brain networks during learning. Proceedings of the National Academy of Sciences 108, 7641-7646, doi:10.1073/pnas.1018985108 (2011). Cole, M. W. et al. Multi-task connectivity reveals flexible hubs for adaptive task control. Nature neuroscience 16, 13481355, doi:10.1038/nn.3470 (2013). Monti, R. P. et al. Estimating time-varying brain connectivity networks from functional MRI time series. Neurolmage 103, 427-443, doi:https://doi.org/10.1016/j.neuroimage.2014.07.033 (2014).

96 Hoekzema, E. et al. An independent components and functional connectivity analysis of resting state fMRI data points to neural network dysregulation in adult ADHD. Human brain mapping 35, 1261-1272, doi:10.1002/hbm.22250 (2014). Bressler, S. L. \& Menon, V. Large-scale brain networks in cognition: emerging methods and principles. Trends in cognitive sciences 14, 277-290, doi:10.1016/j.tics.2010.04.004 (2010).

98 Berkovich-Ohana, A., Glicksohn, J., Ben-Soussan, T. D. \& Goldstein, A. Creativity Is Enhanced by Long-Term Mindfulness Training and Is Negatively Correlated with Trait Default-Mode-Related Low-Gamma Inter-Hemispheric Connectivity. Mindfulness 8, 717-727, doi:10.1007/s12671-016-0649-y (2017).

99 Jung, R., Mead, B., Carrasco, J. \& Flores, R. The structure of creative cognition in the human brain. Frontiers in Human Neuroscience 7, doi:10.3389/fnhum.2013.00330 (2013).

100 Rucker, J. J. H., Iliff, J. \& Nutt, D. J. Psychiatry \& the psychedelic drugs. Past, present \& future. Neuropharmacology 142, 200-218, doi:https://doi.org/10.1016/j.neuropharm.2017.12.040 (2018).

101 Beck, A. T. Depression: Clinical, experimental, and theoretical aspects. (University of Pennsylvania Press, 1967).

102 Nolen-Hoeksema, S., Wisco, B. E. \& Lyubomirsky, S. Rethinking rumination. Perspectives on psychological science 3, 400424 (2008)

103 Bouso, J. C., Doblin, R., Farre, M., Alcazar, M. A. \& Gomez-Jarabo, G. MDMA-assisted psychotherapy using low doses in a small sample of women with chronic posttraumatic stress disorder. Journal of psychoactive drugs 40, 225-236, doi:10.1080/02791072.2008.10400637 (2008).

104 Frecska, E., Bokor, P. \& Winkelman, M. The Therapeutic Potentials of Ayahuasca: Possible Effects against Various Diseases of Civilization. Frontiers in Pharmacology 7, 35, doi:10.3389/fphar.2016.00035 (2016).

\section{Supplemental Information}




\section{Material and Methods}

Participants. Participants were recruited through advertisements around Maastricht University and internet forums in the Netherlands. Inclusion criteria were: age, 18-40 years; previous experience with a psychedelic drug, but not within the past 3 months; normal weight, body mass index between 18 and $28 \mathrm{~kg} / \mathrm{m} 2$; free from psychotropic medication; good physical health, including absence of major medical, endocrine, and neurological conditions; and written informed consent. Exclusion criteria were: history of drug abuse or addiction; pregnancy or lactation; health issues including hypertension (diastolic >90 and systolic >140), cardiac dysfunction, and liver dysfunction; current or history of psychiatric disorders; previous experience of serious side effects to psychedelics; and MRI contraindications. Before inclusion, subjects answered medical questionnaires about their health and drug use, and were screened and examined by a study physician, who checked for general health, conducted a resting ECG, and took blood and urine samples in which hematology, clinical chemistry, urine, and virology analyses were conducted. Participant demographic data can be found in Table S1.

Psilocybin (powder) was obtained from GH Pharm GmbH, Frankfurt, Germany. A permit for obtaining, storing, and administering psilocybin was obtained from the Dutch Drug Enforcement Administration. Participants were financially compensated for their participation in the study.

Procedure. Participants were familiarized with the test day procedures on a separate training day prior to the treatment conditions, where they also completed the baseline creativity tests. Participants were instructed to refrain from drug use, including psychedelic drugs ( $\geq 3$ months), MDMA/ecstasy ( $\geq 14$ days), alcohol ( $\geq 24$ hours), and all other drugs of abuse ( $\geq 7$ days) prior to their first testing day, and to remain sober until completion of the follow-up testing day (7 days later). Additionally, participants were asked to refrain from caffeine and nicotine use the day of the test day.

On arrival of a test day, absence of drug and alcohol use was assessed via a urine drug screen and a breath alcohol screen. An additional pregnancy test was given if participants were female. If all tests were found to be negative, participants were allowed to proceed, and a venal catheter was placed, in order to take blood samples throughout the testing day. Before administration of treatment, a baseline blood sample was taken and baseline vital signs (blood pressure and heart rate) were measured. After measurements, the treatment was administered orally, in a closed cup containing bitter lemon (placebo) or bitter lemon and psilocybin (powder). After 40 minutes, participants were placed in the MRI scanner, where resting state scans and magnetic resonance spectroscopy were performed throughout a 1 hour time window. When they were taken out of the MRI scanner, they returned to the laboratory room where they 
completed the creativity tests. At the end of the test day (approximately 6 hours after treatment administration), participants were asked to complete measures of retrospective subjective high. Participants stayed under supervision until the testing day was complete, and the researcher deemed they were fit to go home.

Participants then returned 7 days later, and were screened again for drug and alcohol use, and if applicable pregnancy, via the aforementioned methods. If the tests were found to be negative, they were allowed to proceed and complete the creativity tests.

\section{Picture Concept Task}

The PCT consists of 17 stimuli, each containing between 4 and 12 color pictures shown in a matrix of $2 \times 2,2 \times 3,3 \times 3$, or $3 \times 4$. Participants were given 30 seconds per stimulus to find an association between one of the pictures in each row. Specifically, they were asked first to provide the correct solution, as there is only one correct answer. The number of correct answers served as the dependent measure of convergent thinking. In order to assess divergent thinking, participants were asked to provide as many alternative answers as possible. This is the regular instruction included in measures of divergent thinking, and it is used to calculate several parameters-i.e., fluency, originality, and the ratio of both-which reflect quantity and quality of divergent thinking. Fluency is defined as the number of alternative associations. The second parameter (i.e., originality) is calculated by evaluating the originality of the alternative association relative to those provided by all other participants in a session. Alternative answers that were uniquely reported by a single participant received an originality score of 2 , answers that were shared with a single participant were valued as 1 , and answers that were shared by three or more participants were rated zero. Originality was the sum of the originality points. In addition, the ratio of originality and fluency was calculated, to correct originality for the number of responses that were generated. When a participant would give two responses of 'medium' originality, which is worth one point, and another participant would give only one highly original response, which is worth two points, Originality would be awarded with two in total for both. Even though the scores are the same, the quality is not. Ratio reflects this difference in quality with the first participant in the example having Ratio ' 1 ' and the second participant Ratio ' 2 '.

\section{AUT}

Three different versions of the AUT were also given to participants. On version A, participants were asked to come up with as many alternative uses as possible for "pen" and "newspaper", version B included the two words "towel" and "bottle", and version C included "brick" and "shoe". Here originality scores were calculated as 2 points if the answer was given by less than $1 \%$ of the participants, and 1 point if the answer was given by less than $5 \%$ of the participants. The AUT can 
also be scored in regards to additional outcome measures ${ }^{1}$, which we decided not to include in this study in order to be as succinct as possible between creativity tasks. These outcome variables include remoteness of association (remote), cleverness of the response (clever), and perceived creativity of the response (creative), flexibility, and elaboration. The ability to generate a diversity of responses 'Flexibility' was assessed by clustering the responses into categories; the sum of categories was the dependent variable. The amount of detail in the responses, 'Elaboration' was scored by summating the number of details.

\section{Questionnaires}

5-Dimensional Altered States of Consciousness Rating Scale. The 5D-ASC is a 94-item self-report scale that assesses the participants' alterations from normal waking consciousness ${ }^{2}$. The participant is asked to make a vertical mark on the $10-\mathrm{cm}$ line below each statement to rate to what extent the statements applied to their experience in retrospect from "No, not more than usually" to "Yes, more than usually." The 5D-ASC contains the 5 key dimensions, including anxious ego dissolution, visual restructuralization, auditory alterations, reduction of vigilance, and oceanic boundlessness; which can be broken down into 11 subscales consisting of experience of unity, spiritual experience, blissful state, insightfulness, disembodiment, impaired control and cognition, anxiety, complex imagery, elementary imagery, audio-visual synesthesia, and changed meaning of percepts

\section{Blood}

All samples were centrifuged and serum was frozen at $-20^{\circ} \mathrm{C}$, and kept in the dark until analysis. Analysis of psilocin in serum was performed according to Martin, et al. ${ }^{3}$. Serum (200 $\mu$ l) was extracted with $1 \mathrm{ml}$ of ethyl acetate after addition of phosphate buffer $\mathrm{pH}$ 9, $20 \mathrm{ng}$ psilocine- $\mathrm{d}_{10}$ and $10 \mu \mathrm{l}$ of $0.1 \mathrm{M}$ ascorbic acid for stabilization. The organic phase was evaporated and reconstituted with $100 \mu \mathrm{l}$ of $0.1 \%$ formic acid/acetonitrile $(80: 20, v / v)$. The analysis of $2 \mu \mathrm{l}$ was performed on an Agilent (Waldbronn, Germany) LC-MS/MS system consisting of a 1290 Infinity Liquid Chromatograph coupled via JetStream Electrospray Interface (ESI) to a G6460A Triple Quadrupole Mass Spectrometer. Analytes were separated on a Kinetex ${ }^{\circledR} 2.6 \mu \mathrm{m}$ XB-C18 $100 \AA$ LC column $(100 \times 2.1 \mathrm{~mm})$ plus corresponding guard column from Phenomenex (Aschaffenburg, Germany) at $30^{\circ} \mathrm{C}$. Gradient elution at a flow rate of $0.5 \mathrm{ml} / \mathrm{min}$ using $0.01 \%$ formic acid containing $5 \mathrm{mM}$ ammonium formate $(\mathrm{A})$ and acetonitrile containing $0.1 \%$ formic acid (B) started with $5 \% \mathrm{~B}$, increased to $95 \% \mathrm{~B}$ during $4 \mathrm{~min}$ and was held for 2 min. Source parameters were: gas temperature $300{ }^{\circ} \mathrm{C}$, gas flow $11 \mathrm{l} / \mathrm{min}$, nebulizer $45 \mathrm{psi}$, sheath gas temperature $400{ }^{\circ} \mathrm{C}$, sheath gas flow $12 \mathrm{l} / \mathrm{min}$ and capillary voltage $3500 \mathrm{~V}$. Detection was performed in the multiple reaction monitoring mode $\left(\mathrm{m} / \mathrm{z}\right.$, collision energy in parentheses, quantifier underlined): psilocine- $\mathrm{d}_{10}$ : $215^{\circledR} 66$ (12), psilocine $\underline{205}^{\circledR} 58(12) ; 205^{\circledR} 160$ (16). Five calibration standards were prepared from 
human serum with psilocine reference substance (LGC Standards GmbH, Wesel, Germany) and analyzed with the samples. The calibration was linear (regression coefficient $>0.999$ ) in the range $1-100 \mathrm{ng} / \mathrm{ml}$ with limits of detection and quantification below $0.5 \mathrm{ng} / \mathrm{ml}$.

\section{Preprocessing.}

Data was processed and analysed using the CONN toolbox 18. $b^{4}$ (http://www.nitrc.org/projects/conn) based on SPM12 (http://www.fil.ion.ucl.ac.uk/spm/) running in MATLAB 2019a. Before quality assessment, FMRI data was available for 26 subjects in the psilocybin group and 27 subjects in the placebo group.

All functional volumes were realigned, unwarped, segmented into grey and white matter and cerebrospinal fluid, normalised into a standard stereotactic space (Montreal Neurological Institute; $\mathrm{MNI}$ ) and smoothed with a $6 \mathrm{~mm}$ full width at half maximum Gaussian kernel. The first two volumes were excluded in order to to ensure magnetization equilibrium. No slice-time correction was performed as data was acquired using multi-band acquisition.

All individual $\mathrm{T}_{1}$-weighted structural volumes were segmented into grey and white matter and cerebrospinal fluid and normalised to MNI space. Noise correction of the functional images included scrubbing with a global signal threshold of $z>3$ and a composite subject motion threshold of $>0.5 \mathrm{~mm}$ using ART as implemented in CONN, linear detrending, linear regression of the six motion parameters, and the white matter and cerebrospinal fluid signals, using the individual tissue masks obtained from the $\mathrm{T}_{1}$-weighted structural images. Five principal components were extracted from white matter and cerebrospinal fluid signals (using individual tissue masks obtained from the T1-weighted structural images) and removed using CompCor ${ }^{5}$. These components are thought to reflect noise (especially motion and physiological fluctuations) and are therefore removed from the time series. The resulting functional images were band-pass filtered $(0.008<\mathrm{f}$ $<0.09 \mathrm{~Hz}$ ) as it was found that band-pass filtering improves independent component results in addition to high-pass filtering (Pignat et al., 2013).

Quality assessment comprised three stages: Firstly, all scans were assessed with regard to the percentage of the scrubbed volumes. Subjects were only included in further analysis, if $\geq 5 \mathrm{~min}$ of the scan remained after scrubbing (corresponding to $<83.6 \%$ of the initial volumes). This was based on literature indicating that resting state scans $<5$ min are not reliable (Birn et al., 2013). Secondly, head motion after scrubbing was assessed using maximum framewise displacement (FD; sphere radius $50 \mathrm{~mm}$ ) calculated according to Power et al. (Power et al., 2012). Subjects were excluded if maximum FD was $>0.75 \mathrm{~mm}$ (half-voxel size). Four subjects (psilocybin group: 3; placebo group: 1) were excluded based on the first criterium and one subject (psilocybin group) 
was excluded based on the second criterium. The final sample thus consisted of 22 subjects in the psilocybin group and 26 subjects in the placebo group. All further analyses were based on this sample. Furthermore, we tested for significant differences between the groups of this sample. Mean FD before scrubbing was $0.18 \mathrm{~mm}( \pm$ SD 0.05) in the psilocybin group and $0.18 \mathrm{~mm}( \pm$ SD $0.06)$ in the placebo group. Average maximum FD before scrubbing in the psilocybin group was $0.98 \mathrm{~mm}( \pm$ SD 0.78) in the psilocybin group and $0.99 \mathrm{~mm}( \pm$ SD 0.95) in the placebo group. On average 10.90 volumes per scan ( \pm SD 9.49) were scrubbed in the psilocybin group and 10.50 volumes per scan ( \pm SD 9.80) in the placebo group. Mean FD after scrubbing was $0.17 \mathrm{~mm}( \pm$ SD $0.05)$ in the psilocybin group and $0.17 \mathrm{~mm}( \pm$ SD 0.05) in the placebo group. Average maximum FD before scrubbing in the psilocybin group was $0.51 \mathrm{~mm}( \pm$ SD 0.12) and $0.50 \mathrm{~mm}( \pm$ SD 0.13$)$ in the placebo group. For comparison between groups, Mann-Whitney $U$ tests were performed because the assumption for normality distribution was not met in several cases (assessed with ShapiroWilk tests). There were no significant differences between groups for any of these measures (invalid volumes: $p=0.77$; mean FD before scrubbing: $p=0.72$; average maximum FD before scrubbing: $p=0.81$; mean FD after scrubbing: $p=0.81$; average maximum FD after scrubbing: $p=0.70)$.

\section{Independent component analysis.}

Independent component analysis (ICA) was performed using group-ICA procedures implemented in the CONN toolbox following methods described by Calhoun et at. (Calhoun et al., 2001). ICA results are determined by the chosen number of dimensions, i.e. a higher number of dimensions might result in a higher number of distinct resting state networks compared with a lower number of dimensions. Dimensionality reduction on the subject-level was set to 64 . Independent components were restricted to 20 in order to allow comparisons with 10 established resting state network described by Smith et al. (Smith et al., 2009) and previous studies on psilocybin (CarhartHarris et al., 2013) and LSD (Carhart-Harris et al., 2016; Müller et al., 2018), which also applied comparable restrictions. Decisions regarding the labelling of the networks identified in this data set were based on visual inspection (Kelly Jr. et al., 2010) and cross-correlation of the unthresholded ICA components with the unthresholded resting state networks described by Smith et al. (https://www.fmrib.ox.ac.uk/datasets/brainmap+rsns/).

\section{Results}

\section{AUT}

Independent samples t-tests revealed an acute significant decrease in flexibility (psilocybin: -3.74 \pm 0.82; placebo: $-1.05 \pm 0.81 ; \mathrm{t}(56)=-2.23, p=.02$ ) elaboration (psilocybin: $-0.64 \pm 0.42$; placebo: 
$0.34 \pm 1.48 ; \mathrm{t}(56)=-1.96, \mathrm{p}=.05$ ), ratings of remoteness (psilocybin: $-.22 \pm 0.05$; placebo: $.002 \pm$ $0.05 ; \mathrm{t}(56)=-3.09, p=.003$ ), clever (psilocybin: $-0.05 \pm 0.05$; placebo: $0.16 \pm 0.04 ; \mathrm{t}(56)=-3.36$, $p=.001$ ), and creativity (psilocybin: $-0.09 \pm 0.04$; placebo: $0.13 \pm 0.05 ; \mathrm{t}(56)=-3.78, p<.001$ ) after psilocybin compared to placebo. At the follow-up, there was a trending significant increase of flexibility (psilocybin: $-1.19 \pm 0.87$; placebo: $-3.24 \pm 0.61 ; \mathrm{t}(56)=-1.93, p=.06$ ), and no change in the other variables (all $p>$.4).

\section{Post-hoc correlation}

A spearman's correlation was conducted post-hoc to investigate the relationship between acute feelings of insight, and long-term changes in new ideas generated on the AUT (novel). A significant positive correlation was found $r_{s}=.296, p=.026$.

Table S1. ICC interrater reliability for all dependent outcome variables on the creativity task, which were scored by 2 independent raters

\begin{tabular}{|l|l|l|l|l|l|l|l|}
\hline & Variable & ICC & $95 \% \mathrm{Cl}$ & $\mathrm{F}$ & $\mathrm{Df1}$ & $\mathrm{Df2}$ & $\mathrm{p}$ \\
\hline \multirow{3}{*}{ AUT } & Originality & .909 & $.867-.941$ & 10.964 & 57 & 285 & .000 \\
\cline { 2 - 8 } & Remote & .884 & $.830-.925$ & 8.607 & 57 & 285 & .000 \\
\cline { 2 - 8 } & Clever & .879 & $.824-.922$ & 8.292 & 57 & 285 & .000 \\
\cline { 2 - 8 } & Creative & .901 & $.855-.936$ & 10.060 & 57 & 285 & .000 \\
\hline PCT & Originality & .947 & $.922-.966$ & 18.921 & 52 & 260 & .000 \\
\hline
\end{tabular}

\section{References}

1 Silvia, P. J. et al. Assessing creativity with divergent thinking tasks: Exploring the reliability and validity of new subjective scoring methods. Psychology of Aesthetics, Creativity, and the Arts 2, 68 (2008).

2 Studerus, E., Gamma, A. \& Vollenweider, F. X. Psychometric evaluation of the altered states of consciousness rating scale (OAV). PLOS ONE 5, e12412 (2010).

3 Martin, R., Schurenkamp, J., Pfeiffer, H. \& Kohler, H. A validated method for quantitation of psilocin in plasma by LCMS/MS and study of stability. International journal of legal medicine 126, 845-849, doi:10.1007/s00414-011-0652-8 (2012).

4 Whitfield-Gabrieli, S. \& Nieto-Castanon, A. Conn: a functional connectivity toolbox for correlated and anticorrelated brain networks. Brain connectivity 2, 125-141, doi:10.1089/brain.2012.0073 (2012).

5 Behzadi, Y., Restom, K., Liau, J. \& Liu, T. T. A component based noise correction method (CompCor) for BOLD and perfusion based fMRI. Neurolmage 37, 90-101, doi:10.1016/j.neuroimage.2007.04.042 (2007). 


\section{Chapter 7}

\section{Discussion}

The main aim of this dissertation was twofold: to investigate whether cannabis and psilocybin acutely influence brain markers that reflect neuroadaptations in key brain areas implicated in the action of these substances, and to investigate if such brain changes predict established acute and persisting drug-specific effects. To that end, several experimental studies were conducted to gain more insight into markers that reflect acute neuroadaptations induced by single and repeated drug exposure, which may underlie previously established behavioral effects of cannabis and psilocybin. Although it is methodologically impossible to assess neuronal function and formation in vivo in humans, magnetic resonance spectroscopy (MRS) is a non-invasive imaging technique that allows quantification of brain markers that reflect neuroadaptations (i.e., glutamate levels). Additionally, resting-state $\mathrm{fMRI}$ can be used to assess alterations in circuit-level interactions between brain regions, as a more systems-level biomarker of drug-induced neuroadaptations ${ }^{44}$.

\section{Acute biological and behavioral evidence that cannabis can induce neuroadaptations}

Cannabis remains the most widely used illicit drug in the world. Although the acute effects of cannabis on subjective and behavioral states are well known, including increased feelings of euphoria and decreased performance in cognitive domains such as attention and memory ${ }^{45-47}$, the neurochemical mechanisms underlying such effects have yet to be fully elucidated. In light of the changing legalization status and a growing interest in the therapeutic utility, it is essential to understand the biochemical alterations that occur during use, as behavioral evidence suggests that cannabis can induce long-lasting neuroadaptations in the brain. Namely, it has been demonstrated that repeated use of cannabis results in behavioral tolerance, e.g., the acute effects of a single administration of cannabis are less prominent in regular users of cannabis ${ }^{9}$. Furthermore, it has been found that $10 \%$ of cannabis users develop problematic daily use patterns ${ }^{48}$. As daily use of cannabis is the standard treatment regimen for therapeutic application, understanding the underlying neuroadaptations involved in these processes is essential for realizing the potential consequences of long-term use in both recreational and clinical users.

Although a majority of studies have focused on the role of dopamine in mediating the behavioral effects of cannabis and other drugs of abuse, the mesocorticolimbic dopamine reward circuitry consists of rich glutamatergic innervation; providing an anatomical basis for interactions between dopamine and glutamate in regulating the rewarding properties of drugs of abuse, as 
well as synaptic plasticity ${ }^{42}$. However, no human studies so far had assessed or demonstrated a role of glutamate during acute cannabis exposure. By applying a multimodal brain imaging strategy, Chapter 2 aimed to assess for the first time whether, and at what dose, cannabis altered glutamate in the nucleus accumbens (NAC) and the anterior cingulate cortex (ACC), two key brain regions involved in the mesocorticolimbic reward circuit. This is of particular importance in the context of substance abuse due to the circuit's involvement in reward, motivation and salience attribution, inhibitory control, and conditioning and memory ${ }^{49,50}$. As demonstrated in Chapter 2, when peak THC levels in serum surpassed $2 \mathrm{ng} / \mathrm{mL}$, THC increased glutamate in the striatum, but not the ACC. Furthermore, THC decreased synchronicity between BOLD responses in the NAC and other brain areas implicated in the mesocorticolimbic circuit, a suggested marker of elevated dopamine release ${ }^{51,52}$. Finally, alterations in NAc glutamate and functional connectivity (FC) within the mesocorticolimbic circuit correlated strongly, emphasizing the intricate interconnectedness of the mesolimbic dopaminergic and glutamatergic system ${ }^{53}$.

Notably, Chapter 2 demonstrated that THC increased a marker that reflects acute neuroadaptations (i.e., glutamate), which was indirectly related to behaviors suggested to be neuroplastic. Specifically, feelings of subjective high and impairment in attention have been found to be blunted in individuals who regularly use cannabis ${ }^{9}$. Thus, due to the findings in Chapter 2 , Chapter 3 extended the study to compare the acute effects of cannabis on glutamate in the NAC and ACC, FC in the mesocorticolimbic system, subjective high, and sustained attention in occasional versus chronic cannabis users. Here, the original sample from Chapter 2 that received the single dose of $300 \mathrm{mcg} \mathrm{THC} / \mathrm{kg}$ body weight was increased by 2, to allow for a final comparison between 12 occasional and 12 chronic users. As expected, in Chapter 3, the same results were found as in the occasional users in Chapter 2. However, in the chronic users there was an absence of cannabis-induced increase in striatal glutamate and stimulation of the reward system, as well as mitigation of behavioral alterations. The finding that cannabis altered the reward circuitry and distorted behavior in occasional, but not chronic users, suggests the development of neuroadaptations in the reward circuitry after excessive use of cannabis, which may reduce the behavioral response to acute cannabis impairment.

\section{Mechanisms which may underlie the "blunted" high}

Due in part to the findings in Chapter 2 and 3, a pharmacodynamic model of neuroadaptive changes in the brain was proposed, to explain a blunted response to cannabis in regular users ${ }^{54}$. Namely, it was suggested that in occasional users of cannabis, activation of CB1 receptors by an acute challenge with THC reduces the inhibitory tone from GABAergic neurons to the ventral tegmental area (VTA), leading to more dopamine release in the NAc ${ }^{13}$. The subsequent increase in cannabis-induced dopamine release is then associated with a loss of functional connectivity within 
the mesocorticolimbic circuit, as demonstrated in Chapter 2 and a previous study by our group ${ }^{52}$. The increase in dopamine release in the mesocorticolimbic circuit then disinhibits thalamic signaling. Thereafter increasing glutamatergic signaling to the prefrontal cortex, VTA and the $\mathrm{NAc}^{52}$, which subsequently stimulates the circuit even more, as evident from the strong correlation between NAC glutamate and mesolimbic FC found in Chapter 2. In chronic cannabis users, however, acute exposure to cannabis or THC does not decrease the inhibitory tone from GABAergic neurons to the VTA, potentially due to the downregulation and desensitization of CB1 receptors in cortical and subcortical regions after repeated exposure to $\mathrm{THC}^{55-62}$. Thus, the absence of change in FC of the NAc with other parts of the reward circuit, as demonstrated in Chapter 3, could suggest that downregulation of CB1 receptors mitigates the impact of acute cannabis intoxication on neural activity within fronto-subcortical circuits and associated behavioral outcomes, demonstrating a potential prime mechanism underlying the development of tolerance in this circuit.

Of note, Chapter 2 and $\mathbf{3}$ did not find a direct relationship between glutamate changes and changes in behavior in occasional users. Instead, there was a strong correlation between changes in mesocorticolimbic FC and changes in behavior. If changes in mesocorticolimbic FC are indicative of enhanced dopaminergic neurotransmission as hypothesized, then this relationship is in line with previous studies that have found that drug-induced increases in activity of mesolimbic dopaminergic neurons mediate the rewarding effects of cannabis and other drugs of abuse ${ }^{43,63-68}$. That said, the strong correlation between increases in dopaminergic neurotransmission and increases in NAc glutamate levels, suggests that alterations in glutamate perhaps play a more indirect role in regards to manifestations of behaviors that display tolerance, like the blunting of the rewarding effects. Evidence for this indirect role comes from preclinical studies assessing the other manifestation of drug-induced neuroplasticity, e.g., compulsive drug-seeking behavior ${ }^{69}$. Here a range of preclinical evidence suggests that glutamate's stimulatory interaction with the mesolimbic dopaminergic system plays a significant role in processes underlying the development of addiction, such as reinforcement learning and processes maintaining addiction, such as craving and relapse ${ }^{53}$.

Additionally, it has been found that blocking glutamatergic (and subsequently dopaminergic) transmission in the VTA and NAc, attenuates the rewarding effects of drugs of abuse $^{68}$. Finally, it has been found that regulating plasticity in the NAc through the control of glutamatergic signaling is an effective way to inhibit drug-seeking to the majority of drugs of abuse $^{70,71}$. Overall, this suggests that glutamate's significant role regarding behavioral effects of drugs of abuse such as cannabis, is its direct or indirect modification of the activity of the dopaminergic system ${ }^{53}$. 


\section{Acute biological and behavioral evidence that psilocybin can induce neuroadaptations}

Although psychedelic substances have been used for hundreds, and perhaps thousands, of years ${ }^{72}$, recently there is an emerging interest in their ability to elicit persisting (positive) changes in clinical and non-clinical populations ${ }^{10}$. Namely, a growing number of clinical trials suggest that psychedelics can provide fast-acting and long-term relief after only a few doses, for a range of psychiatric disorders that are increasingly common and difficult to treat ${ }^{73}$. Thus, an important question is how these substances work in the brain to elicit such effects. Most of the focus of psychedelics' action has been on the role of 5- $\mathrm{HT}_{2 \mathrm{~A}}$ stimulation in the acute experience, which is the main mechanism of action of the hallucinogenic effects ${ }^{14,20}$. That said, preclinical studies suggest that activation of $5-\mathrm{HT}_{2 \mathrm{~A}}$ receptors leads to a glutamate-dependent increase in the activity of pyramidal neurons in the prefrontal cortex, which are also implicated in the acute effects of the $\operatorname{drug}^{74-79}$.

Additionally, this increase in extracellular glutamate has been suggested to activate AMPA receptors located on the same neurons, increasing the expression of BDNF, which is hypothesized to drive neuroplastic adaptations ${ }^{19,20,38,39}$. In line with this, recent preclinical studies have demonstrated psychedelics' capacity to induce functional and structural neuroplasticity ${ }^{80-82}$. Taken together, the findings suggest that $5-\mathrm{HT}_{2 \mathrm{~A}}$ receptor-mediated glutamate release is a common pathway for the acute actions of psychedelics, and glutamate-induced neuroadaptations may underlie long-term therapeutic effects ${ }^{19}$. That said, whether the neuroplastic effects of psychedelics observed in animal studies are replicable in humans and are responsible for longterm effects seen in recent clinical studies requires further investigation ${ }^{20}$.

By using a similar study design as the previous chapters, Chapter 4 aimed to address this knowledge gap by utilizing MRS to assess the influence of a moderate dose of psilocybin on glutamate levels in the medial prefrontal cortex (mPFC) and the hippocampus, two brain areas implicated as potential key regions in modulating the psychedelic experience ${ }^{74-77,83-89}$. In accordance with preclinical studies ${ }^{74,90}$, it was found that those who were administered psilocybin demonstrated higher levels of glutamate in the MPFC, and lower levels of glutamate in the hippocampus, compared to the placebo condition; providing indirect evidence that psychedelics might have the potential to increase neuroplasticity in the human cortex via increased glutamatergic activity, but not in the hippocampus. It was also found that psilocybin reduced within-network FC of several large-scale networks, including the default mode network, visual networks, and the auditory network, while increasing between-network FC. To assess the functional relevance of such brain changes, relevant biological variables such as glutamate in the 
MPFC and hippocampus, and within-network FC of the DMN were used to investigate if they could predict a key feature of the psychedelic state, e.g., the experience of ego dissolution. It was found that higher levels of mPFC glutamate were the strongest predictor of negatively experienced ego dissolution, whereas lower levels in hippocampal glutamate were found to be the strongest predictor of positively experienced ego dissolution. Such findings could be relevant in explaining biochemical mechanisms of long-term change, as it has been found that the "quality" of the acute psychedelic experience predicts long-term changes. Namely, the more positively experienced ego dissolution acutely -- which is frequently compared to a peak or mystical experience -- the better individuals feel in the long-term ${ }^{32,91-93}$. Thus findings suggest the potential to predict long-term behavior changes from acute biological changes. That said, further insight can be gained by focusing on behaviors in which psychedelic drugs have been more directly demonstrated to induce persisting effects on.

\section{A case for creativity}

One of the arguably longest-standing behavioral associations with psychedelic drugs is their supposed ability to enhance creativity. Famous examples of psychedelic-affiliated creative breakthroughs span the field of science, technology, and art. They include Kary Mullis' discovery of the polymerase chain reaction, the 1960's California based computer industry, and the literary works of authors such as Aldous Huxley and Ken Kesey ${ }^{6,94}$. Although there has been much historical interest in the ability of psychedelics to (sub)acutely enhance creative capacity, the scientific literature is mostly lacking. Chapter 5 aimed to investigate these notions in a naturalistic setting. Attendants of a psilocybin ceremony were tested on facets of creativity, i.e., divergent ("flexible") and convergent ("logical") thinking, both the morning after and seven days after intake. It was found that psilocybin increased divergent thinking (DT) the morning after use, and increased convergent thinking (CT) 7 days post use, compared to baseline. Due to the inherent limitations of a naturalistic design, such as a lack of placebo control, and uncontrolled non-pharmacological factors such as psychological expectations (set) and environment (setting), known to play an essential role in the outcome of a psychedelic experience ${ }^{95-98}$, Chapter 6 aimed to replicate and extend the findings from the naturalistic setting in a controlled environment, and to assess biological markers which may underlie behavioral changes. Using the same creativity task as in Chapter 5 (Picture Concept Test, PCT), and including the "gold standard" task in assessing DT (Alternate Uses Test, AUT) as well as a rating of a domain akin to spontaneous creative "insight", findings from Chapter 6 demonstrated that psilocybin acutely decreased performance on various measure of task-based creativity, including the outcome measure of CT on the PCT, and outcome measures of DT on both tasks. Conversely, subjective ratings of spontaneous creativity (i.e., "insight") were increased acutely. Seven days later, the number of novel ideas individuals in the 
psilocybin group could come up with on the AUT was increased, compared to placebo. Combining the findings from Chapter 5 and 6 , it is suggested that psychedelics do not just "enhance creativity" as anecdotal reports have claimed, but rather induce a time- and construct-related differentiation of effects on creative thinking. Findings suggest that frameworks conceptualizing the impact of the psychedelic state on creativity should be adapted to include both acute and persisting effects of the drug ${ }^{99}$.

It was found that both acute and persisting performance on the creativity measurements in Chapter 6 were predicted by within- and between-network connectivity of the DMN. Namely, levels of acute connectivity between both subcomponents of the DMN and the fronto-parietal network ("task-based", FPN) were found to inversely predict both acute and long-term changes in CT performance, i.e., increasingly positive DMN-FPN connectivity predicted increasingly worse CT performance relative to placebo. Additionally, decreased within-network DMN FC predicted both an acute decrease in originality scores and a long-term increase in the generation of novel ideas. Although this finding may initially seem counterintuitive, it adds evidence to suggest that psychedelics induce lasting neuroadaptations. Namely, given the suggested role of the DMN in underlying the idea generation process of DT ${ }^{100-102}$, an acute decrement in DMN FC resulting in an acute decrement in DT performance on a creativity task would be expected. That said, previous work has found that while psychedelics decrease within-network DMN FC acutely, they increase DMN integrity sub- acutely ${ }^{103-106}$, potentially via the aforementioned neuroplastic effect on brain network function. Thus, it could be suggested that the latter prediction seen here is reflecting the subsequent psilocybin-induced increase in DMN FC that could facilitate an increase in novel ideas.

Regarding the ability of glutamate to directly predict performance in creative cognition measures, it was found that decreased glutamate in the hippocampus was a secondary predictor of acute ratings of insight, the latter which interestingly (positively) correlated with the number of novel ideas 7 days later. Conversely, although the role of the mPFC has been strongly highlighted in creative cognition ${ }^{107}$, glutamate levels in this area did not play a statistically significant role. This suggests that the involvement of the mPFC is via its role in the integration and interaction of distributed brain areas operating in large-scale networks ${ }^{108}$, highlighting the complementary information that can be gained when applying multimodal imaging paradigms to assess druginduced neuroadaptations.

\section{Mind-bending, heart-mending? Implications and caveats on defining} "(ab)use"

The chapters mentioned above provide evidence that cannabis and psilocybin acutely induce neuroadaptations, which may have lasting consequences on brain function and behavior. Notably, 
such behavioral consequences can be either beneficial (adaptive) or detrimental, causing unwanted symptoms or behavioral patterns (maladaptive). That said, the threshold between what is "good" and "bad" is subjective, and arguably highly dependent on the motivation to use the substance in the first place.

Recreational users of cannabis report using the substance for the rewarding effects, including feelings of euphoria and relaxation ${ }^{3}$. In this regard, the development of tolerance and subsequent blunting of such effects can be seen as maladaptive, as they will have to use more of the drug to reach their desired state. Importantly, as prolonged exposure to cannabis progressively blunts reward system function, this can lead to escalated frequency and amount of cannabis being used, resulting in a more physiologically dependent state; thus, a risk of the development of tolerance is that it could also promote drug addiction ${ }^{109}$. However, cannabis tolerance is not a permanent state that is reached after chronic cannabis use, but rather a temporary state of decreased sensitivity, dependent on frequency of cannabis consumption ${ }^{54}$. Thus, a technique used by regular users of cannabis is to take "tolerance breaks", reducing the quantity and frequency of cannabis use, to lower cannabis tolerance. However, the time required to reverse tolerance, especially given previous frequency and dose, are currently unknown. The demographics of users in Chapters 2 and 3 who did not display tolerance reported a lower frequency of use (mean (SD) use of 1.21 (0.80) times a week), compared to the cannabis-using group who displayed tolerance, whom used the drug almost every day (6.63 (1.20) times a week). In comparing brain and behavioral responses of these two groups to an acute dose of cannabis, it provides a relatively broad spectrum in that using cannabis about 1 time a week may not blunt the rewarding effects of the drug, whereas using almost every day does. If excessive use does happen, previous studies assessing for example the CB1 receptor availability have found normalization in cannabisdependent users after as little as 2 days of monitored abstinence ${ }^{60,62}$ and neuropsychological data suggesting reversible cognitive deficits, modulated more by recent exposure than by cumulative lifetime use ${ }^{110}$. Thus it could be suggested that by controlling the frequency of use (for example, using 1 time a week versus every day) or by taking tolerance breaks (refraining from use for at least 2 days), one can control the impact of cannabis-induced neuroadaptations. However, the literature on long-lasting effects of cannabis use is mixed, with studies also reporting long-term changes on brain structure ${ }^{111,112}$, neurometabolite levels ${ }^{113}$ and neurocognitive functioning ${ }^{114}$, which may differ depending on the THC-content (i.e. potency) of the cannabis used $^{115}$. Importantly, results of the aforementioned studies have been found to be brain regionand domain-specific and influenced by other factors such as frequency and age of onset of use, potentially explaining the variance in reported outcomes. Future studies should take such factors into account to further assess brain and behavioral consequences of repeated cannabis exposure on the reward system. 
Furthermore, with the release of pharmaceutical cannabis-based medicines and the liberalization of cannabis access, the issue of cannabis impairment and tolerance will gain importance in cannabis therapeutics as well ${ }^{54}$. However, here, the rewarding effects of cannabis could be seen as unwanted, and adverse neurocognitive impairment can be dangerous for individuals who must use the product daily and still function normally (e.g., drive to work). Thus, in these situations, the development of tolerance that leads to immunity of such behavioral effects could be seen as adaptive. Indeed, dosing strategies in patient populations have been recommended to promote tolerance, including slow upward dose titration and low doses of cannabis to attain symptom control while avoiding the impairing effects ${ }^{116}$. That said, effective dose regimens that promote or avoid cannabis tolerance are presently unknown. In our sample in Chapter 2, cannabis only induced brain changes, which may underlie tolerance of THC's rewarding and impairing effects, after peak blood levels of THC surpassed $2 \mathrm{ng} / \mathrm{mL}$. Such a study design provides a potential threshold for what cannabis dose induces such (mal)adaptive neuroadaptations in the brain. Future studies can employ a similar paradigm, assessing markers of drug-induced neuroadaptations in different patient and user populations, with varying histories of cannabis use, to determine threshold doses that produce an optimal balance between clinical benefits and adverse behavioral side effects.

Regarding psilocybin, the finding that psilocybin acutely induced region-dependent alterations in glutamate, which correlated with "positive" and "negative" aspects of the psychedelic experience, makes it tempting to suggest that by selectively (pharmacologically) modulating glutamate release, one could tailor the experience of the drug. However, ubiquitous distribution of glutamate makes targeting glutamate transmission to decrease or increase the specific effects of any drug very challenging ${ }^{68}$. Further, it should be emphasized that glutamate transmission is involved in many other physiological functions such as learning and memory ${ }^{68}$, and as previously mentioned, it is this enhancement of glutamate in the MPFC that is suggested to facilitate neuroplasticity and thus maintain long-term effects of the drug. Instead, perhaps it could be suggested that understanding the neurobiological mechanisms of such substances can provide insight into how to tailor the behavioral experience.

For example, the finding that psilocybin induces region-dependent alterations in glutamate, and thus potentially localization of enhanced neuroplasticity, as well as the disintegration of established resting-state networks, may provide insight into the cognitive and learning enhancements that could take place after ingestion of a psychedelic. Specifically, as addressed by Carhart-Harris and Nutt ${ }^{117}$, several studies have shown enhancements of associative learning with $5-\mathrm{HT}_{2 \mathrm{~A}}$ receptor agonism, and impairments with its blockade. Additionally, it has been well established that the MPFC suppresses limbic activity (see Carhart and Friston ${ }^{118}$ for an overview), potentially via glutamatergic projections from the mPFC terminating on inhibitory 
interneurons in the medial temporal lobes ${ }^{119,120}$, a pattern which has been implicated in the extinction of associations ${ }^{120}$. Thus it could be suggested that via region-dependent modulation of the glutamatergic system, associative learning is enhanced via psychedelics, and new connections could be made, subsequently replacing previously formed associations. Future studies could assess the ability of psilocybin to locally induce neuroplasticity and ultimately change behavior by utilizing a similar paradigm as Chapter 4 and 6; using MRS to assess acute markers of neuroadaptations (glutamate) in select brain regions, and then following up to investigate the potential evolution of brain plastic changes over time. To do this, a combination of more persisting markers of neuroadaptation could be investigated, such as neurochemical markers, e.g., NAcetylaspartate, a marker of neuronal viability and function that can be quantified with MRS $^{121}$ in combination with techniques to assess functional plasticity (e.g., resting-state fMRI) and structural plasticity, such as quantifying grey matter volume or cortical thickness ${ }^{122}$. Such changes could then be related to repeated, long-term behavioral assessments.

The previous paragraph highlights the inherent value-neutral quality of the cognitive and learning enhancements which could take place after ingestion of a psychedelic, in that the type of associations that are being learned and/or replaced could be either adaptive or maladaptive, and may depend on the motivation to take the drug in the first place. As previously stated, a common motivation for recreational users, and an aim for clinical populations, is to use a psychedelic to increase creative, flexible thinking and gain new insights and perspectives of previous events and current problems ${ }^{94,123}$. Namely, the mental health disorders psychedelics are currently being investigated to treat are characterized by rigid, inflexible thought patterns, which may drive habitual behaviors $88,124,125$. Thus taking into consideration the findings from Chapter 5 and 6 , it could be suggested that the ability of psilocybin to acutely decrease CT and increase spontaneous DT, while subacutely enhancing more goal-directed DT, could aid in the therapeutic process by opening up a window of opportunity where therapeutic interventions could prove more effective. Specifically, while under the influence of a psychedelic, rigid thought content (CT) is decreased, while unguided, spontaneous thoughts (and associative learning) is enhanced. Subacutely (24 hours -1 week later), patients may then be able to integrate these insights with a therapist and come up with new, more effective strategies that facilitate adaptive interpretation and coping abilities $^{126}$. Thus, understanding acute and persisting psilocybin-induced neuroadaptations and subsequent malleability in cognitive processes such as creative thinking could prove to facilitate adaptive use regarding designing treatment plans to maximize the effectiveness of psychedelic use. 


\section{Concluding the "good", the "bad", and the intoxicated}

In conclusion, the data mentioned above demonstrate that cannabis and psilocybin acutely induce region-dependent alterations in glutamate that correlate with established biological and behavioral changes during the intoxicated state. Such findings provide further insights into the underlying neurobiological mechanisms of the intoxicated state, and importantly, provide a neurochemical basis for how these substances may be giving rise to both adaptive and maladaptive behavioral effects. As the definition of (ab)use is dependent on motivations to use the substance, understanding mechanisms underlying such effects can perhaps allow for insights on how to tailor the experience for each population, depending on the desired outcome of the drug experience. 


\section{References}

1 Merlin, M. D. Archaeological evidence for the tradition of psychoactive plant use In the old world. Economic Botany 57, 295-323, doi:10.1663/0013-0001(2003)057[0295:AEFTTO]2.0.CO;2 (2003).

2 Osborne, G. B. \& Fogel, C. Understanding the motivations for recreational marijuana use among adult Canadians. Substance use \& misuse 43, 539-572; discussion 573-539, 585-537, doi:10.1080/10826080701884911 (2008).

3 Kettner, H., Mason, N. L. \& Kuypers, K. P. C. Motives for classical and novel psychoactive substances use of psychedelic polydrug-users Contemporary Drug Problems (2019).

4 Mason, N. L. \& Kuypers, K. P. Mental health of a self-selected sample of psychedelic users and self-medication practices with psychedelics. Journal of Psychedelic Studies 2, 45-52 (2018).

5 Hutten, N. R. P. W., Mason, N. L., Dolder, P. C. \& Kuypers, K. P. C. Motives and Side-Effects of Microdosing With Psychedelics Among Users. The international journal of neuropsychopharmacology 22, 426-434, doi:10.1093/ijnp/pyz029 (2019).

6 Baggott, M. J. Psychedelics and creativity: a review of the quantitative literature. PeerJ PrePrints 3, e1202v1201, doi:10.7287/peerj.preprints.1202v1 (2015).

$7 \quad$ Nutt, D., Erritzoe, D. \& Carhart-Harris, R. Psychedelic Psychiatry's Brave New World. Cell 181, 24-28 (2020).

8 Montero-Oleas, N., Arevalo-Rodriguez, I., Nuñez-González, S., Viteri-García, A. \& Simancas-Racines, D. Therapeutic use of cannabis and cannabinoids: an evidence mapping and appraisal of systematic reviews. BMC complementary medicine and therapies 20, 12 (2020).

9 Colizzi, M. \& Bhattacharyya, S. Cannabis use and the development of tolerance: a systematic review of human evidence. Neurosci Biobehav Rev 93, 1-25, doi:10.1016/j.neubiorev.2018.07.014 (2018).

Aday, J. S., Mitzkovitz, C. M., Bloesch, E. K., Davoli, C. C. \& Davis, A. K. Long-term effects of psychedelic drugs: A systematic review. Neuroscience \& Biobehavioral Reviews 113, 179-189, doi:https://doi.org/10.1016/j.neubiorev.2020.03.017 (2020).

11 Nestler, E. J. Is there a common molecular pathway for addiction? Nature neuroscience 8, 1445-1449 (2005).

12 Kalivas, P. W. \& O'Brien, C. Drug Addiction as a Pathology of Staged Neuroplasticity. Neuropsychopharmacology 33, 166180, doi:10.1038/sj.npp.1301564 (2008).

13 Bloomfield, M. A., Ashok, A. H., Volkow, N. D. \& Howes, O. D. The effects of Delta9-tetrahydrocannabinol on the dopamine system. Nature 539, 369-377, doi:10.1038/nature20153 (2016). Nichols, D. E. Psychedelics. Pharmacological reviews 68, 264-355, doi:10.1124/pr.115.011478 (2016). Gardoni, F. \& Bellone, C. Modulation of the glutamatergic transmission by Dopamine: a focus on Parkinson, Huntington and Addiction diseases. Frontiers in cellular neuroscience 9, 25, doi:10.3389/fncel.2015.00025 (2015). Ciranna, L. Serotonin as a modulator of glutamate- and GABA-mediated neurotransmission: implications in physiological functions and in pathology. Curr Neuropharmacol 4, 101-114, doi:10.2174/157015906776359540 (2006).

Pehrson, A. L. \& Sanchez, C. Serotonergic modulation of glutamate neurotransmission as a strategy for treating depression and cognitive dysfunction. CNS spectrums 19, 121-133, doi:10.1017/s1092852913000540 (2014). Boto, T. \& Tomchik, S. M. The Excitatory,
the Brain. Neuron 101, 763-765 (2019) Vollenweider, F. X. \& Kometer, M. The neurobiology of psychedelic drugs: implications for the treatment of mood disorders. Nature reviews. Neuroscience 11, 642-651, doi:10.1038/nrn2884 (2010). Vollenweider, F. X. \& Preller, K. H. Psychedelic drugs: neurobiology and potential for treatment of psychiatric disorders. Nature Reviews Neuroscience, doi:10.1038/s41583-020-0367-2 (2020). systematic review of human and animal evidence. Neuroscience and Biobehavioral Reviews 64, 359-381, doi:10.1016/j.neubiorev.2016.03.010 (2016).

doi:10.1016/0301-0082(90)90013-7 (1990).

Cramer, S. C. et al. Harnessing neuroplasticity for clinical applications. Brain : a journal of neurology 134, 1591-1609, doi:10.1093/brain/awr039 (2011). 31-39, doi:10.1038/361031a0 (1993). Seo, D. \& Sinha, R. Neuroplasticity and Predictors of Alcohol Recovery. Alcohol research : current reviews 37, 143-152 (2015). Sharma, N., Classen, J. \& Cohen, L. G. Neural plasticity and its contribution to functional recovery. Handb Clin Neurol 110, 3-12, doi:10.1016/B978-0-444-52901-5.00001-0 (2013).
Carhart-Harris, R. L. et al. Psilocybin with psychologic feasibility study. The Lancet Psychiatry 3, 619-627 (2016). 


\section{Chapter 7}

Griffiths, R. R., Richards, W. A., McCann, U. \& Jesse, R. Psilocybin can occasion mystical-type experiences having substantial and sustained personal meaning and spiritual significance. Psychopharmacology (Berl) 187, 268-283; discussion 284-292, doi:10.1007/s00213-006-0457-5 (2006).

Griffiths, R. R., Richards, W. A., Johnson, M. W., McCann, U. D. \& Jesse, R. Mystical-type experiences occasioned by psilocybin mediate the attribution of personal meaning and spiritual significance 14 months later. Journal of psychopharmacology 22, 621-632 (2008).

Johnson, M. W., Garcia-Romeu, A. \& Griffiths, R. R. Long-term follow-up of psilocybin-facilitated smoking cessation. The American journal of drug and alcohol abuse 43, 55-60, doi:10.3109/00952990.2016.1170135 (2017).

Uthaug, M. V. et al. Sub-acute and long-term effects of ayahuasca on affect and cognitive thinking style and their association with ego dissolution. Psychopharmacology, 1-11 (2018).

Griffiths, R. R. et al. Psilocybin produces substantial and sustained decreases in depression and anxiety in patients with life-threatening cancer: A randomized double-blind trial. Journal of Psychopharmacology 30, 1181-1197, doi:10.1177/0269881116675513 (2016).

Gasser, P. et al. Safety and efficacy of lysergic acid diethylamide-assisted psychotherapy for anxiety associated with lifethreatening diseases. The Journal of nervous and mental disease 202, 513-520, doi:10.1097/NMD.0000000000000113 (2014).

Palhano-Fontes, F. et al. Rapid antidepressant effects of the psychedelic ayahuasca in treatment-resistant depression: a randomized placebo-controlled trial. Psychological medicine 49, 655-663, doi:10.1017/s0033291718001356 (2019).

Grob, C. S. et al. Pilot study of psilocybin treatment for anxiety in patients with advanced-stage cancer. Archives of general psychiatry 68, 71-78, doi:10.1001/archgenpsychiatry.2010.116 (2011).

Sanches, R. F. et al. Antidepressant Effects of a Single Dose of Ayahuasca in Patients With Recurrent Depression: A SPECT Study. Journal of clinical psychopharmacology 36, 77-81, doi:10.1097/jcp.0000000000000436 (2016).

Vollenweider, F. X. \& Kometer, M. The neurobiology of psychedelic drugs: Implications for the treatment of mood disorders. Nature Reviews Neuroscience 11, 642-651, doi:http://dx.doi.org/10.1038/nrn2884 (2010).

Vaidya, V. A., Marek, G. J., Aghajanian, G. K. \& Duman, R. S. 5-HT2A receptor-mediated regulation of brain-derived neurotrophic factor mRNA in the hippocampus and the neocortex. The Journal of neuroscience : the official journal of the Society for Neuroscience 17, 2785-2795 (1997).

Cavus, I. \& Duman, R. S. Influence of estradiol, stress, and 5-HT2A agonist treatment on brain-derived neurotrophic factor expression in female rats. Biological psychiatry 54, 59-69 (2003).

Ramaekers, J. G., Kauert, G., Theunissen, E., Toennes, S. W. \& Moeller, M. Neurocognitive performance during acute THC intoxication in heavy and occasional cannabis users. Journal of psychopharmacology 23, 266-277 (2009).

Ramaekers, J. G. et al. Tolerance and cross-tolerance to neurocognitive effects of THC and alcohol in heavy cannabis users. Psychopharmacology (Berl) 214, 391-401 (2011).

Gass, J. T. \& Olive, M. F. Glutamatergic substrates of drug addiction and alcoholism. Biochem Pharmacol 75, 218-265, doi:10.1016/j.bcp.2007.06.039 (2008).

Koob, G. F. \& Volkow, N. D. Neurocircuitry of addiction. Neuropsychopharmacology 35, 217-238, doi:10.1038/npp.2009.110 (2010).

Sutherland, M. T., McHugh, M. J., Pariyadath, V. \& Stein, E. A. Resting state functional connectivity in addiction: Lessons learned and a road ahead. Neurolmage 62, 2281-2295, doi:10.1016/j.neuroimage.2012.01.117 (2012).

Green, B., Kavanagh, D. \& Young, R. Being stoned: a review of self-reported cannabis effects. Drug and alcohol review 22, 453-460, doi:10.1080/09595230310001613976 (2003).

Ramaekers, J. G., Berghaus, G., van Laar, M. \& Drummer, O. H. Dose related risk of motor vehicle crashes after cannabis use. Drug and alcohol dependence 73, 109-119 (2004).

Hall, W. \& Solowij, N. Adverse effects of cannabis. Lancet (London, England) 352, 1611-1616, doi:10.1016/s01406736(98)05021-1 (1998).

WHO. The Health and Social Effects of Nonmedical Cannabis Use. (2016).

Volkow, N. D., Wang, G. J., Fowler, J. S., Tomasi, D. \& Telang, F. Addiction: beyond dopamine reward circuitry. Proc Natl Acad Sci U S A 108, 15037-15042, doi:10.1073/pnas.1010654108 (2011).

Pierce, R. C. \& Kumaresan, V. The mesolimbic dopamine system: the final common pathway for the reinforcing effect of drugs of abuse? Neurosci Biobehav Rev 30, 215-238, doi:10.1016/j.neubiorev.2005.04.016 (2006).

Ramaekers, J. et al. Methylphenidate reduces functional connectivity of nucleus accumbens in brain reward circuit. Psychopharmacology (Berl) 229, 219-226, doi:10.1007/s00213-013-3105-x (2013).

Ramaekers, J. G. et al. Cannabis and cocaine decrease cognitive impulse control and functional corticostriatal connectivity in drug users with low activity DBH genotypes. Brain Imaging and Behavior 10, 1254-1263 (2016).

Tzschentke, T. M. \& Schmidt, W. J. Glutamatergic mechanisms in addiction. Molecular psychiatry 8, 373-382, doi:10.1038/sj.mp.4001269 (2003). 
Ramaekers, J. G., Mason, N. L. \& Theunissen, E. L. Blunted highs: Pharmacodynamic and behavioral models of cannabis tolerance. European Neuropsychopharmacology 36, 191-205, doi:https://doi.org/10.1016/j.euroneuro.2020.01.006 (2020).

Breivogel, C. S. et al. Chronic delta9-tetrahydrocannabinol treatment produces a time-dependent loss of cannabinoid receptors and cannabinoid receptor-activated G proteins in rat brain. Journal of neurochemistry 73, 2447-2459 (1999). Sim-Selley, L. J. Regulation of cannabinoid CB1 receptors in the central nervous system by chronic cannabinoids. Critical reviews in neurobiology 15, 91-119 (2003).

McKinney, D. L. et al. Dose-related differences in the regional pattern of cannabinoid receptor adaptation and in vivo tolerance development to delta9-tetrahydrocannabinol. The Journal of pharmacology and experimental therapeutics 324, 664-673, doi:10.1124/jpet.107.130328 (2008). on cannabinoid-stimulated [35S]GTPgammaS autoradiography in rat brain. The Journal of neuroscience : the official journal of the Society for Neuroscience 16, 8057-8066 (1996).

Romero, J. et al. Atypical location of cannabinoid receptors in white matter areas during rat brain development. Synapse 26, 317-323 (1997). Hirvonen, J. et al. Reversible and regionally selective downregulation of brain cannabinoid CB1 receptors in chronic daily cannabis smokers. Molecular psychiatry 17, 642-649, doi:10.1038/mp.2011.82 (2012). Addict Biol 20, 357-367, doi:10.1111/adb.12116 (2015).

D'Souza, D. C. et al. Rapid Changes in CB1 Receptor Availability in Cannabis Dependent Males after Abstinence from Cannabis. Biological psychiatry. Cognitive neuroscience and neuroimaging 1, 60-67, doi:10.1016/j.bpsc.2015.09.008 (2016).

Gessa, G. L., Melis, M., Muntoni, A. L. \& Diana, M. Cannabinoids activate mesolimbic dopamine neurons by an action on cannabinoid CB1 receptors. European journal of pharmacology 341, 39-44 (1998).

$\mathrm{Ng}$ Cheong Ton, J. M. et al. The effects of delta 9-tetrahydrocannabinol on potassium-evoked release of dopamine in the rat caudate nucleus: an in vivo electrochemical and in vivo microdialysis study. Brain Res 451, 59-68 (1988). Wise, R. A. The role of reward pathways in the development of drug dependence. Pharmacology \& therapeutics 35, $227-$ 263, doi:10.1016/0163-7258(87)90108-2 (1987). Koob, G. F. Neural mechanisms of drug reinforcement. Annals of the New York Academy of Sciences 654, 171-191, doi:10.1111/j.1749-6632.1992.tb25966.x (1992). doi:10.1016/j.neuron.2012.10.021 (2012). 404, doi:10.3389/fnins.2015.00404 (2015). O'Brien, C. P. Neuroplasticity in addictive disorders. Dialogues in clinical neuroscience 11, 350-353 (2009).

Scofield, M. et al. The nucleus accumbens: mechanisms of addiction across drug classes reflect the importance of glutamate homeostasis. Pharmacological reviews 68, 816-871 (2016).

Kalivas, P. W. The glutamate homeostasis hypothesis of addiction. Nature Reviews Neuroscience 10, 561-572 (2009). Hofmann, A. LSD: my problem child. Psychedelic Reflections, 24 (1980).

Nutt, D. \& Carhart-Harris, R. The current status of psychedelics in psychiatry. JAMA psychiatry (2020).

Aghajanian, G. K. \& Marek, G. J. Serotonin, via 5-HT2A receptors, increases EPSCs in layer V pyramidal cells of prefrontal cortex by an asynchronous mode of glutamate release. Brain Research 825, 161-171, doi:https://doi.org/10.1016/S00068993(99)01224-X (1999).

Aghajanian, G. \& Marek, G. Serotonin induces excitatory postsynaptic potentials in apical dendrites of neocortical pyramidal cells. Neuropharmacology 36, 589-599 (1997).

Puig, M. V., Celada, P., Díaz-Mataix, L. \& Artigas, F. In vivo modulation of the activity of pyramidal neurons in the rat medial prefrontal cortex by 5-HT2A receptors: relationship to thalamocortical afferents. Cerebral Cortex 13, 870-882 (2003).

Béïque, J.-C., Imad, M., Mladenovic, L., Gingrich, J. A. \& Andrade, R. Mechanism of the 5-hydroxytryptamine 2A receptormediated facilitation of synaptic activity in prefrontal cortex. Proceedings of the National Academy of Sciences 104, 98709875 (2007).

Scruggs, J. L., Patel, S., Bubser, M. \& Deutch, A. Y. DOI-Induced activation of the cortex: dependence on 5-HT2A heteroceptors on thalamocortical glutamatergic neurons. Journal of Neuroscience 20, 8846-8852 (2000).

Scruggs, J. L., Schmidt, D. \& Deutch, A. Y. The hallucinogen 1-[2,5-dimethoxy-4-iodophenyl]-2-aminopropane (DOI) increases cortical extracellular glutamate levels in rats. Neuroscience letters 346, 137-140, doi:https://doi.org/10.1016/S0304-3940(03)00547-0 (2003).

Ly, C. et al. Psychedelics Promote Structural and Functional Neural Plasticity. Cell reports 23, 3170-3182, doi:10.1016/j.celrep.2018.05.022 (2018). 


\section{Chapter 7}

81

Catlow, B. J., Song, S., Paredes, D. A., Kirstein, C. L. \& Sanchez-Ramos, J. Effects of psilocybin on hippocampal neurogenesis and extinction of trace fear conditioning. Experimental brain research 228, 481-491, doi:10.1007/s00221-013-3579-0 (2013).

Zhang, G. et al. Stimulation of serotonin $2 \mathrm{~A}$ receptors facilitates consolidation and extinction of fear memory in C57BL/6J mice. Neuropharmacology 64, 403-413 (2013).

Andrade, R. Serotonergic regulation of neuronal excitability in the prefrontal cortex. Neuropharmacology 61, 382-386, doi:10.1016/j.neuropharm.2011.01.015 (2011).

Puig, M. V. \& Gulledge, A. T. Serotonin and prefrontal cortex function: neurons, networks, and circuits. Mol Neurobio/ 44, 449-464, doi:10.1007/s12035-011-8214-0 (2011).

Bombardi, C. \& Di Giovanni, G. Functional anatomy of 5-HT2A receptors in the amygdala and hippocampal complex: relevance to memory functions. Experimental brain research 230, 427-439, doi:10.1007/s00221-013-3512-6 (2013).

Vollenweider, F. X. et al. Positron emission tomography and fluorodeoxyglucose studies of metabolic hyperfrontality and psychopathology in the psilocybin model of psychosis. Neuropsychopharmacology 16, 357-372, doi:10.1016/s0893133x(96)00246-1 (1997).

Carhart-Harris, R. L. \& Friston, K. J. The default-mode, ego-functions and free-energy: a neurobiological account of Freudian ideas. Brain : a journal of neurology 133, 1265-1283, doi:10.1093/brain/awq010 (2010).

Carhart-Harris, R. L. et al. The entropic brain: a theory of conscious states informed by neuroimaging research with psychedelic drugs. Front Hum Neurosci 8 (2014).

Lebedev, A. V. et al. Finding the self by losing the self: Neural correlates of ego-dissolution under psilocybin. Human brain mapping 36, 3137-3153, doi:10.1002/hbm.22833 (2015).

Marek, G. J. Interactions of Hallucinogens with the Glutamatergic System: Permissive Network Effects Mediated Through Cortical Layer V Pyramidal Neurons. Current topics in behavioral neurosciences 36, 107-135, doi:10.1007/7854_2017 480 (2018).

Roseman, L., Nutt, D. J. \& Carhart-Harris, R. L. Quality of acute psychedelic experience predicts therapeutic efficacy of psilocybin for treatment-resistant depression. Frontiers in Pharmacology 8, 974 (2018).

Uthaug, M. V. et al. A single inhalation of vapor from dried toad secretion containing 5-methoxy-N,N-dimethyltryptamine (5-MeO-DMT) in a naturalistic setting is related to sustained enhancement of satisfaction with life, mindfulness-related capacities, and a decrement of psychopathological symptoms. Psychopharmacology (Berl) 236, 2653-2666, doi:10.1007/s00213-019-05236-w (2019).

MacLean, K. A., Johnson, M. W. \& Griffiths, R. R. Mystical experiences occasioned by the hallucinogen psilocybin lead to increases in the personality domain of openness. Journal of Psychopharmacology 25, 1453-1461 (2011).

Sessa, B. Is it time to revisit the role of psychedelic drugs in enhancing human creativity? Journal of Psychopharmacology 22, 821-827 (2008).

Eisner, B. Set, setting, and matrix. Journal of psychoactive drugs 29, 213-216, doi:10.1080/02791072.1997.10400190 (1997).

Hartogsohn, I. Constructing drug effects: A history of set and setting. Drug Science, Policy and Law 3, 2050324516683325, doi:10.1177/2050324516683325 (2017).

Leary, T., Litwin, G. H. \& Metzner, R. Reactions to psilocybin administered in a supportive environment. The Journal of nervous and mental disease 137, 561-573 (1963).

Metzner, R. \& Leary, T. On programming psychedelic experiences. Psychedelic Review 9, 5-19 (1967).

Girn, M., Mills, C., Roseman, L., Carhart-Harris, R. L. \& Christoff, K. Updating the dynamic framework of thought: Creativity and psychedelics. Neurolmage 213, 116726, doi:https://doi.org/10.1016/j.neuroimage.2020.116726 (2020).

Beaty, R. E., Benedek, M., Barry Kaufman, S. \& Silvia, P. J. Default and Executive Network Coupling Supports Creative Idea Production. Scientific Reports 5, 10964, doi:10.1038/srep10964 (2015).

Beaty, R. E., Benedek, M., Silvia, P. J. \& Schacter, D. L. Creative Cognition and Brain Network Dynamics. Trends in cognitive sciences 20, 87-95, doi:10.1016/j.tics.2015.10.004 (2016).

Beaty, R. E. et al. Robust prediction of individual creative ability from brain functional connectivity. Proceedings of the National Academy of Sciences 115, 1087-1092, doi:10.1073/pnas.1713532115 (2018).

Sampedro, F. et al. Assessing the Psychedelic "After-Glow" in Ayahuasca Users: Post-Acute Neurometabolic and Functional Connectivity Changes Are Associated with Enhanced Mindfulness Capacities. International Journal of Neuropsychopharmacology 20, 698-711, doi:10.1093/ijnp/pyx036 (2017).

Carhart-Harris, R. L. et al. Psilocybin for treatment-resistant depression: fMRI-measured brain mechanisms. Scientific Reports 7, 13187, doi:10.1038/s41598-017-13282-7 (2017).

Smigielski, L., Scheidegger, M., Kometer, M. \& Vollenweider, F. X. Psilocybin-assisted mindfulness training modulates selfconsciousness and brain default mode network connectivity with lasting effects. Neurolmage 196, 207-215 (2019).

Barrett, F. S., Doss, M. K., Sepeda, N. D., Pekar, J. J. \& Griffiths, R. R. Emotions and brain function are altered up to one month after a single high dose of psilocybin. Scientific Reports 10, 2214, doi:10.1038/s41598-020-59282-y (2020).

Dietrich, A. The cognitive neuroscience of creativity. Psychonomic bulletin \& review 11, 1011-1026 (2004). 
108 Bressler, S. L. \& Menon, V. Large-scale brain networks in cognition: emerging methods and principles. Trends in cognitive sciences 14, 277-290, doi:10.1016/j.tics.2010.04.004 (2010).

109 Parsons, L. H. \& Hurd, Y. L. Endocannabinoid signalling in reward and addiction. Nature reviews. Neuroscience 16, 579594, doi:10.1038/nrn4004 (2015).

110 Pope, H. G., Jr, Gruber, A. J., Hudson, J. I., Huestis, M. A. \& Yurgelun-Todd, D. Neuropsychological Performance in Longterm Cannabis Users. JAMA Psychiatry 58, 909-915, doi:10.1001/archpsyc.58.10.909 (2001).

111 Filbey, F. M. et al. Long-term effects of marijuana use on the brain. Proceedings of the National Academy of Sciences 111 , 16913-16918, doi:10.1073/pnas.1415297111 (2014).

112 Battistella, G. et al. Long-term effects of cannabis on brain structure. Neuropsychopharmacology 39, 2041-2048, doi:10.1038/npp.2014.67 (2014).

113 Sneider, J. T., Mashhoon, Y. \& Silveri, M. M. A Review of Magnetic Resonance Spectroscopy Studies in Marijuana using Adolescents and Adults. Journal of addiction research \& therapy Suppl 4.

114 Crean, R. D., Crane, N. A. \& Mason, B. J. An evidence based review of acute and long-term effects of cannabis use on executive cognitive functions. Journal of addiction medicine 5, 1-8, doi:10.1097/ADM.0b013e31820c23fa (2011).

115 Rigucci, S. et al. Effect of high-potency cannabis on corpus callosum microstructure. Psychological medicine 46, 841-854, doi:10.1017/s0033291715002342 (2016).

116 MacCallum, C. A. \& Russo, E. B. Practical considerations in medical cannabis administration and dosing. European journal of internal medicine 49, 12-19, doi:10.1016/j.ejim.2018.01.004 (2018).

117 Carhart-Harris, R. \& Nutt, D. Serotonin and brain function: a tale of two receptors. Journal of Psychopharmacology 31, 1091-1120 (2017).

118 Carhart-Harris, R. L. \& Friston, K. J. The default-mode, ego-functions and free-energy: a neurobiological account of Freudian ideas. Brain 133, 1265-1283, doi:10.1093/brain/awq010 (2010).

119 Rosenkranz, J. A. \& Grace, A. A. Cellular mechanisms of infralimbic and prelimbic prefrontal cortical inhibition and dopaminergic modulation of basolateral amygdala neurons in vivo. The Journal of neuroscience : the official journal of the Society for Neuroscience 22, 324-337 (2002).

120 Rosenkranz, J. A., Moore, H. \& Grace, A. A. The prefrontal cortex regulates lateral amygdala neuronal plasticity and responses to previously conditioned stimuli. The Journal of neuroscience: the official journal of the Society for Neuroscience 23, 11054-11064 (2003).

121 Manji, H. K., Moore, G. J., Rajkowska, G. \& Chen, G. Neuroplasticity and cellular resilience in mood disorders. Molecular psychiatry 5, 578-593, doi:10.1038/sj.mp.4000811 (2000).

122 Zatorre, R. J., Fields, R. D. \& Johansen-Berg, H. Plasticity in gray and white: neuroimaging changes in brain structure during learning. Nature neuroscience 15, 528-536, doi:10.1038/nn.3045 (2012).

123 Kuypers, K. P. C. Out of the box: A psychedelic model to study the creative mind. Medical hypotheses 115, 13-16 (2018). Beck, A. T. Depression: Clinical, experimental, and theoretical aspects. (University of Pennsylvania Press, 1967).

125 Nolen-Hoeksema, S., Wisco, B. E. \& Lyubomirsky, S. Rethinking rumination. Perspectives on psychological science 3, 400424 (2008).

126 Kuypers, K. P. C. et al. Ayahuasca enhances creative divergent thinking while decreasing conventional convergent thinking. Psychopharmacology (Berl) 233, 3395-3403, doi:10.1007/s00213-016-4377-8 (2016). 


\section{Summary}

Humans have been looking for ways to alter their consciousness by taking drugs that are found in nature since time immemorial. Today, substances like cannabis and psychedelics are used by many people, for a myriad of recreational and therapeutic reasons. Importantly, as both recreational and therapeutic interest into these substances grows, so will use. Thus a pertinent question is how these substances work to elicit such effects, and what the long-term consequences of use are. The latter is of particular importance, as single doses of these substances have been suggested to produce neuroadaptations that underlie acute and long-term (mal)adaptive behavioral changes. As the underlying neuroadaptations giving rise to such effects have not been systematically assessed in humans, several experimental studies were conducted in order to gain more insight into markers that reflect acute neuroadaptations induced by single and repeated drug exposure, which may underlie previously established behavioral effects of cannabis and psilocybin.

The study in Chapter 2 aimed to assess whether, and at what dose, cannabis altered glutamate levels in key brain regions implicated in action of drugs of abuse (the NAc and ACC), and whether this related with established brain and behavior outcomes. By employing a double-blind, placebo-controlled twenty occasional cannabis users received acute doses of cannabis ( $300 \mu \mathrm{g} / \mathrm{kg}$ THC) and placebo, in one of two dose regimes (full dose and divided dose), during two separate testing days. Using MRS and MRI, levels of glutamate and GABA, and (indirect) dopaminergic neurotransmission were quantified and their relationship with behavioral outcome measures was assessed, namely increases in subjective high, and decreases in sustained attention. It was found that administration of THC increased striatal glutamate levels, and dopamine as indicated by a reduction in functional connectivity (FC) between the NAc and cortical areas. Alterations in glutamate and FC were dose dependent and evident in the full dose group where THC serum concentrations exceeded $2 \mathrm{ng} / \mathrm{mL}$ at T-max. Average glutamate changes correlated strongly with FC alterations. Additionally, THC induced changes in FC correlated with feelings of subjective high and decreased performance on an attention task. Taken together, the findings suggest that THC elicits subjective and cognitive alterations via increased striatal dopaminergic activity and loss of corticostriatal connectivity, which is associated with an increase in striatal glutamate.

Due to the previous results demonstrating the acute ability of cannabis to influence markers that reflect neuroadaptations, Chapter 3 extended the study to compare the acute effects of cannabis on glutamate in the NAC and ACC, FC in the mesocorticolimbic system, and subjective high and sustained attention in two distinct cannabis user groups. In this double-blind, randomized, placebo-controlled, cross-over study, 12 occasional and 12 chronic cannabis users received acute doses of cannabis $(300-\mu \mathrm{g} / \mathrm{kg}$ delta-9-tetrahydrocannabinol) and placebo and 
underwent ultrahigh field functional magnetic resonance imaging and magnetic resonance spectroscopy. In occasional users, cannabis induced significant neurometabolic alterations in reward circuitry, namely, decrements in functional connectivity and increments in striatal glutamate levels, which were associated with increases in subjective high and decreases in performance on a sustained attention task. Such changes were absent in chronic users. The finding that cannabis altered the reward circuitry and distorted behavior in occasional, but not chronic users, suggests reduced responsiveness of the reward circuitry to cannabis intoxication in chronic users, suggesting a pharmacodynamic mechanism for the development of tolerance to cannabis impairment.

By using a similar study design as in the previous chapters, Chapter 4 aimed to assess whether psilocybin had the ability to acutely influence markers that reflect neuroadapations (e.g. glutamate) in key brain regions implicated in the psychedelic experience. Following a double-blind, placebo-controlled, parallel group design, it was demonstrated that psilocybin $(0.17 \mathrm{mg} / \mathrm{kg})$ induced region-dependent alterations in glutamate, which predicted distortions in the subjective experience of one's self (ego dissolution). Whereas higher levels of medial prefrontal cortical glutamate were associated with negatively experienced ego dissolution, lower levels in hippocampal glutamate were associated with positively experienced ego dissolution. Such findings provide further insights into the underlying neurobiological mechanisms of the psychedelic state. Importantly, as positively experienced ego dissolution has been found to predict long-term increases in well-being, they may also provide a neurochemical basis for therapeutic effects as witnessed in ongoing clinical trials.

A long-standing behavioral effect that has been suggested to be induced by psychedelic use are (sub)acute enhancements in creative thinking. Chapter 5 aimed to confirm these notions in a naturalistic setting, where attendants of a psilocybin ceremony were tested on constructs of creativity, i.e. divergent ("flexible") and convergent ("logical") thinking, as well as empathy and subjective well-being, both the morning after $(n=50)$, and 7 days after $(n=22)$ intake. Results indicated that compared to baseline psilocybin enhanced divergent thinking and emotional empathy the morning after use. Enhancements in convergent thinking, valence-specific emotional empathy, and well-being persisted seven days after use. Interestingly, sub-acute changes in empathy correlated with changes in well-being.

As the previous chapter demonstrated that a single administration of psilocybin may be associated with persisting enhancements of creative thinking, Chapter 6 aimed to replicate and extend the findings from the naturalistic setting in a controlled environment, and to assess biological markers which may underlie behavioral changes. As part of the same double-blind, placebo-controlled, parallel-group design in Chapter 4, results demonstrated that psilocybin (0.17 
$\mathrm{mg} / \mathrm{kg}$ ) induced a time- and construct-related differentiation of effects on creative thinking. Acutely, psilocybin increased ratings of (spontaneous) creative insights, while decreasing (deliberate) task-based creativity. Seven days after psilocybin, the number of novel ideas increased. Furthermore, it was found that acute and persisting effects were predicted by withinand between-network connectivity of the default mode network. Overall, findings add support to historical claims that psychedelics can facilitate aspects of the creative process, and provide evidence of acute neuroadaptations that may underlie the (sub)acute effects.

Finally, in Chapter 7 the key findings are discussed in a broader context. Namely, it was demonstrated that cannabis and psilocybin acutely induce region dependent alterations in glutamate, that correlate with established biological and behavioral changes during the intoxicated state. Such findings provide further insights into the underlying neurobiological mechanisms of the intoxicated state, and importantly, provide a neurochemical basis for how these substances may be giving rise to both adaptive and maladaptive behavioral effects. Importantly, as the definition of (ab)use is dependent on motivations to use the substance, implications for the utility of these findings for both recreational users, and clinical practitioners, are considered. 


\section{Samenvatting}

Mensen zijn al sinds mensenheugenis op zoek naar manieren om hun bewustzijn te veranderen door drugs te gebruiken die in de natuur voorkomen. Vandaag de dag worden stoffen als cannabis en psychedelica door veel mensen gebruikt voor een groot aantal recreatieve en therapeutische redenen. Belangrijk is, dat naarmate de recreatieve en therapeutische belangstelling voor deze stoffen toeneemt, het gebruik ervan ook toeneemt. Een relevante vraag is dus hoe deze stoffen werken om dergelijke effecten uit te lokken en wat de langetermijngevolgen van het gebruik zijn. Dit laatste is met name belangrijk, aangezien gesuggereerd wordt dat een enkele dosis van deze stoffen neuroadaptaties veroorzaakt, die ten grondslag liggen aan acute en lange termijn (on)aangepaste gedragsveranderingen. Omdat de onderliggende neuroadaptaties die aanleiding geven tot dergelijke effecten niet systematisch onderzocht zijn bij mensen, zijn er verschillende experimentele studies uitgevoerd om meer inzicht te krijgen in de kenmerken die de acute neuroadaptaties, veroorzaakt door eenmalige en herhaalde blootstelling aan drugs, weerspiegelen en zo aan de basis kunnen liggen van eerder vastgestelde gedragseffecten van cannabis en psilocybine.

De studie in hoofdstuk 2 was bedoeld om te beoordelen of, en bij welke dosis, cannabis het glutamaatniveau in de belangrijkste hersengebieden betrokken bij de werking van drugs (de $\mathrm{NAc}$ en ACC) veranderde en of dit verband hield met vastgestelde hersen- en gedragsuitkomsten. Door middel van een dubbelblind, placebogecontroleerde studie, kregen twintig incidentele cannabisgebruikers gedurende twee afzonderlijke testdagen een acute dosis cannabis ( $300 \mu \mathrm{g} / \mathrm{kg}$ $\mathrm{THC}$ ) en placebo in een van de twee dosesregimes (volledige dosis of verdeelde dosis). Met behulp van MRS en MRI werden de niveaus van glutamaat en GABA en (indirect) dopaminerge neurotransmissie gekwantificeerd en werd hun relatie met gedragsmatige uitkomstmaten beoordeeld, namelijk toename van de subjectieve high en afname van volgehouden aandacht. Het bleek dat toediening van THC leidde tot een toename in striatale glutamaatniveaus en dopamine zoals aangetoond door een vermindering van de functionele connectiviteit (FC) tussen de NAc en corticale gebieden. Veranderingen in glutamaat en FC waren dosisafhankelijk en duidelijk in de volledige dosisgroep waar de THC-serumconcentraties hoger waren dan $2 \mathrm{ng} / \mathrm{mL}$ bij T-max. De gemiddelde veranderingen in glutamaat waren sterk gecorreleerd met de veranderingen in FC. Bovendien veroorzaakte THC veranderingen in FC gecorreleerd met gevoelens van subjectieve high en verminderde prestatie op een aandachtstaak. Alles bij elkaar genomen suggereren de bevindingen dat THC subjectieve en cognitieve veranderingen teweegbrengt via verhoogde striatale dopaminerge activiteit en verlies van corticostriatale connectiviteit, welke geassocieerd worden met een toename van striataal glutamaat. 
Aangezien de voorgaande resultaten het acute vermogen van cannabis om kenmerken die neuroadaptaties weerspiegelen te beïnvloeden aantoonden, werd de studie in hoofdstuk 3 uitgebreid met een vergelijking van de acute effecten van cannabis op glutamaat in de NAc en ACC, FC in het mesocorticolimbische systeem en subjectieve high en volgehouden aandacht in twee verschillende cannabisgebruikersgroepen. In deze dubbelblinde, gerandomiseerde, placebogecontroleerde cross-over studie, kregen 12 incidentele en 12 chronische cannabisgebruikers acute doses cannabis (300- $\mu \mathrm{g} / \mathrm{kg}$ delta-9-tetrahydrocannabinol) en placebo en ondergingen ze 'ultrahigh field functional magnetic resonance imaging' en magnetische resonantiespectroscopie. Bij incidentele gebruikers veroorzaakte cannabis significante neurometabolische veranderingen in het beloningscircuit, namelijk een afname van de functionele connectiviteit en een toename van de striatale glutamaatniveaus, die in verband werden gebracht met een toename van de subjectieve high en een afname van de prestaties op een volgehouden aandachtstaak. Dergelijke veranderingen waren afwezig bij chronische gebruikers. De bevinding dat cannabis het beloningscircuit veranderde en het gedrag vervormde bij incidentele, maar niet bij chronische gebruikers, suggereert een verminderd reactievermogen van het beloningscircuit op cannabisintoxicatie bij chronische gebruikers, wat suggereert dat er een farmacodynamisch mechanisme voor de ontwikkeling van tolerantie voor cannabisintoxicatie bestaat.

Het doel van hoofdstuk 4 was, door gebruik te maken van een vergelijkbare studieopzet als in de vorige hoofdstukken, te beoordelen of psilocybine het vermogen had om kenmerken van neuroadapaties (bijv. glutamaat) in belangrijke hersengebieden die betrokken zijn bij de psychedelische ervaring acuut te beïnvloeden. Na een dubbelblind, placebo-gecontroleerd, parallelle groepsopzet werd aangetoond dat psilocybine $(0.17 \mathrm{mg} / \mathrm{kg}$ ) regio-afhankelijke veranderingen in glutamaat veroorzaakte, hetgeen vervormingen in de subjectieve ervaring van iemands zelf (ego-oplossing) voorspelde. Terwijl hogere niveaus van glutamaat in de mediale prefrontale cortex werden geassocieerd met negatief ervaren "ego dissolution" (ontbinding van het ego), werden lagere niveaus glutamaat in de hippocampus geassocieerd met positief ervaren ontbinding van het ego. Dergelijke bevindingen geven meer inzicht in de onderliggende neurobiologische mechanismen van de psychedelische toestand. Het is belangrijk dat, aangezien positief ervaren ontbinding van het ego lange termijn verbetering van het welzijn voorspelde, het ook een neurochemische basis voor therapeutische effecten kan zijn, zoals blijkt uit lopende klinische studies.

Een reeds lang bestaand gedragseffect dat wordt gesuggereerd door psychedelisch gebruik is (sub)acute verbetering in het creatieve denken. Hoofdstuk 5 had tot doel deze ideeën te bevestigen in een naturalistische setting, waarbij de deelnemers aan een psilocybine ceremonie werden getest op concepten van creativiteit, d.w.z. divergent ("flexibel") en convergent ("logisch") denken, evenals empathie en subjectief welzijn, en dit zowel de ochtend na $(n=50)$, als 7 dagen na 
$(n=22)$ inname. De resultaten geven aan dat, in vergelijking met het basisniveau, psilocybine het divergent denken en de emotionele empathie de ochtend na gebruik versterkt. De verbeteringen in convergent denken, valentie-specifieke emotionele empathie en welzijn hielden zeven dagen na gebruik aan. Interessant is dat subacute veranderingen in empathie gecorreleerd zijn met veranderingen in welzijn.

Aangezien het vorige hoofdstuk aantoonde dat een enkele toediening van psilocybine geassocieerd kan worden met aanhoudende verbeteringen van het creatieve denken, was hoofdstuk 6 gericht op het repliceren en uitbreiden van de bevindingen uit de naturalistische omgeving in een gecontroleerde omgeving en op het beoordelen van biologische kenmerken die aan de basis kunnen liggen van gedragsveranderingen. Als onderdeel van dezelfde dubbelblinde, placebo-gecontroleerde, parallelle groepsopzet in hoofdstuk 4, toonden de resultaten aan dat psilocybine $(0,17 \mathrm{mg} / \mathrm{kg})$ een tijd- en concept gerelateerde differentiatie van effecten op het creatief denken induceerde. Acute psilocybine verhoogde de waardering van (spontane) creatieve inzichten, terwijl het de (bewuste) taakgerichte creativiteit verminderde. Zeven dagen na toediening van psilocybine nam het aantal nieuwe ideeën toe. Verder bleek dat acute en aanhoudende effecten voorspeld werden door de connectiviteit binnen en tussen het defaultnetwerk. In het algemeen ondersteunen de bevindingen de historische beweringen dat psychedelica aspecten van het creatieve proces kunnen faciliteren en leveren ze bewijs voor acute neuroadaptaties die aan de basis kunnen liggen van (sub)acute effecten.

Tot slot worden in hoofdstuk 7 de belangrijkste bevindingen in een bredere context besproken. Er werd aangetoond dat cannabis en psilocybine acuut regio-afhankelijke veranderingen in glutamaat veroorzaken die correleren met vastgestelde biologische- en gedragsveranderingen tijdens de geïntoxiceerde toestand. Dergelijke bevindingen geven meer inzicht in de onderliggende neurobiologische mechanismen van de geïntoxiceerde toestand en, wat belangrijk is, bieden een neurochemische basis voor de manier waarop deze stoffen aanleiding kunnen geven tot zowel aangepaste als onaangepaste gedragseffecten. Belangrijk is dat, aangezien de definitie van drug gebruik of misbruik afhankelijk is van motivaties om de stof te gebruiken, de implicaties voor de bruikbaarheid van deze bevindingen voor zowel recreatieve gebruikers, als voor de klinische praktijk, worden overwogen. 


\section{Impact paragraph}

The findings in this thesis suggest that a single dose of cannabis or psilocybin can produce brain changes that can result in acute and persisting (mal)adaptive behavioral consequences. As these substances are used by many, for a myriad of recreational and therapeutic purposes, such findings have a wide societal relevance for a range of target groups, including clinicians, policy-makers, scientists, and users alike.

Potential clinical impact. A growing number of clinical trials are looking at the therapeutic utility of psychedelic substances like psilocybin. Importantly, it has been proposed that the "quality" of the psychedelic experience, and in particular the amount of positively experienced ego dissolution, is an indicator of therapeutic success of the substances ${ }^{1,2}$. Information from Chapter 4 provides insight into mechanisms that may underlie the quality of the psychedelic experience, in that it was found that aspects of a "good" and "bad" trip are differentially associated to regional changes in glutamate. It could be proposed that baseline glutamate levels, in combination with baseline functional connectivity ${ }^{3}$ and personality factors ${ }^{4}$, could be used as individual predictive markers of the quality of the psychedelic experience. Subsequently, a better understanding of individual responses could eventually help doctors evaluate which patients would benefit from psychedelic treatment, and could contribute to a personalized medicine approach within the framework of psychedelic-assisted therapy ${ }^{3}$.

The increasing trend in the recreational and therapeutic use of cannabis raises concerns about its impairing effects on performance of day-to-day activities. Understanding the neurobiological basis of such effects is essential in the context of therapeutic use of cannabis, as it is often prescribed daily. An example of the potential utility of this information could stem from Chapter 2, in that it was found that cannabis did not change the brain or induce impairing behavioral effects until cannabis concentrations surpassed a certain threshold in blood. This combination of brain, behavior, and peripheral (i.e. blood concentration) information could be used to inform clinicians on how to prescribe the substance to maximize efficacy and decrease risk, by, for example, prescribing a dosing-scheme of the drug which does not surpass the peripheral threshold known to be associated with the brain changes underlying unwanted effects.

Potential impact for policy-makers. That said, if such a dosing threshold is surpassed, evidence suggests regular cannabis users develop tolerance to the rewarding and impairing effects of the drug. Findings from Chapter 2 and 3 provide a possible measurable biomarker of tolerance. By having such an objective tool to quantify a subjective state, future research can systematically study at what dose and use frequency is necessary to produce tolerance. These results can then 
provide information on how to assess the safety of cannabis use when performing day-to-day operations, and evaluating impairment in legal settings, by for example taking cannabis use history into account to predict impairing behavioral changes (or lack thereof).

Potential impact for recreational users. Additionally, by extending the investigations above to assess how long it takes for the suggested biomarker of tolerance to disappear (i.e. during abstinence), this information could inform recreational users who view tolerance as unwanted, as to how long and often they should take tolerance breaks for. The latter part of is particular importance, as a risk of the development of tolerance is that it could promote drug addiction ${ }^{103}$.

Potential impact for streamlining future research. Furthermore, a perhaps often overlooked (and underutilized) facet of recreational drug use, is that there are many individuals using these substances, and they are using them for a reason. For example, plant-based psychedelics have been used for potentially thousands of years for healing purposes ${ }^{5}$ and there remains an active culture of self-medication with psychedelics for mental health purposes, and in the facilitation of self-exploration ${ }^{6-9}$. Thus there is a wealth of information to be gained from recreational users on potential positive and negative effects of these substance. By collecting data from individuals already using these substances (utilizing naturalistic study designs as in Chapter 5, or questionnaires, examples of which were not included in this thesis $6,7,10-12$ ), one can establish indications of utility of such substances, and further confirm this in clinical or experimental trials. Chapter 6 is an example of this potential process. It extended the findings from the naturalistic setting suggesting that psilocybin can affect different facets of creativity (Chapter 5), and brought it into the lab to replicate, and assess potential underlying biological markers. Findings from Chapter 6 now suggest that psilocybin can be used as a tool in future studies, to perturb everyday creative thinking, and systematically investigate underlying neural mechanisms of the creative process; a process which has proven to be an elusive aspect of cognition to study for neuroscientists.

Current impact. A great deal of effort was put into disseminating the findings from these chapters amongst both science communicators and the general public. Findings from all chapters have, and continue to be, presented at various international scientific conferences. Additionally, all findings have been shared widely across popular social platforms such as Reddit and Twitter, have been covered by newspapers from around the world, and have been presented at meetings of the general public, ranging from invited lectures with university student groups, to informal presentations in local bars. The findings in Chapter 4 and 5 have also been ranked in the top 5\% of all research outputs scored by Altmetric (368 and 276, accordingly). Chapter 5 was submitted for the special $50^{\text {th }}$-anniversary edition of the Journal of Psychoactive Drugs, and quickly became the most-read article in the history of the journal. Additionally, findings from Chapter 4 were added to 
the Wikipedia page of "psilocybin". Due to the potential implications of such findings, and the investment of time, effort, and cost of everyone involved (researchers and participants alike), the dissemination of the findings presented in these chapters with both the scientific community and the general public, will continue to be heavily pursued.

\section{References}

$1 \quad$ Griffiths, R. R. et al. Psilocybin produces substantial and sustained decreases in depression and anxiety in patients with life-threatening cancer: A randomized double-blind trial. Journal of Psychopharmacology 30, 1181-1197, doi:10.1177/0269881116675513 (2016).

2 Roseman, L., Nutt, D. J. \& Carhart-Harris, R. L. Quality of acute psychedelic experience predicts therapeutic efficacy of psilocybin for treatment-resistant depression. Frontiers in Pharmacology 8, 974 (2018).

3 Preller, K. H. et al. Psilocybin induces time-dependent changes in global functional connectivity. Biological psychiatry (2020).

4 Studerus, E., Gamma, A., Kometer, M. \& Vollenweider, F. X. Prediction of psilocybin response in healthy volunteers. PLoS ONE 7, e30800, doi:10.1371/journal.pone.0030800 (2012).

$5 \quad H o f m a n n$, A. LSD: my problem child. Psychedelic Reflections, 24 (1980).

6 Kettner, H., Mason, N. L. \& Kuypers, K. P. C. Motives for classical and novel psychoactive substances use of psychedelic polydrug-users Contemporary Drug Problems (2019).

7 Mason, N. L. \& Kuypers, K. P. Mental health of a self-selected sample of psychedelic users and self-medication practices with psychedelics. Journal of Psychedelic Studies 2, 45-52 (2018).

8 Carhart-Harris, R. L. \& Goodwin, G. M. The Therapeutic Potential of Psychedelic Drugs: Past, Present, and Future. Neuropsychopharmacology 42, 2105-2113, doi:10.1038/npp.2017.84 (2017).

9 Nutt, D., Erritzoe, D. \& Carhart-Harris, R. Psychedelic Psychiatry's Brave New World. Cell 181, $24-28$ (2020).

10 Hutten, N. R., Mason, N. L., Dolder, P. C. \& Kuypers, K. P. Self-rated effectiveness of microdosing with psychedelics for mental and physical health problems amongst microdosers. Frontiers in psychiatry 10, 672 (2019).

11 Hutten, N. R. P. W., Mason, N. L., Dolder, P. C. \& Kuypers, K. P. C. Motives and Side-Effects of Microdosing With Psychedelics Among Users. The international journal of neuropsychopharmacology 22, 426-434, doi:10.1093/ijnp/pyz029 (2019).

12 Mason, N. L., Dolder, P. C. \& Kuypers, K. P. Reported effects of psychedelic use on those with low well-being given various emotional states and social contexts. Drug Science, Policy and Law 6, 2050324519900068 (2020). 


\section{Acknowledgements}

The completion of this dissertation would not have been possible without a team. First and foremost, I would like to acknowledge my supervisor dream-team, prof. dr. Jan Ramaekers and dr. Kim Kuypers. Now I have to mention that, although your acknowledgement comes first, it was written last. This was not out of lack of things to say, but out of an inability to properly verbalize the sheer amount of gratitude I have for you both. Now it goes without saying that I am extremely grateful to you for the support you've given me through this time; you were both always readily available for questions and discussions, and always open to listening to my ideas, no matter how prematurely formulated they may have been. That said, it is all of the other things, the things outside of the "supervisor role" which you didn't have to do, for which I feel especially indebted to you both. Namely, you've given me so many opportunities to grow, and you've put so much trust in me, that it has also helped me to trust and believe in myself. Jan, I've been told from a range of different people how lucky I am to have you as a supervisor, and I couldn't agree more. You always have an open and nonjudgmental ear, willing to listen to all, regardless of years of expertise. You're also always calm, cool, and collected- a much-needed reminder that the academic life doesn't always have to be stressful (and lo and behold can even be tons of fun). I think these qualities are extremely supportive for insecure, young researchers, like myself. I know that through our interactions and conversations, you've helped me build a lot of confidence for myself and my capabilities, of which I will take with me in academia \& beyond. Kim, you are simply a powerhouse of a woman; your ambition is contagious, motivating, and inspiring, and you've become a role model whom I look up to immensely, as well as a friend I can confide in. I could really go on, but the fact of the matter is, I've known for a long time that I want to be a researcher, but under the guidance of the two of you, I'm proud to say that I've become one. For that, I am beyond thankful.

Second, I would like to extend my thanks to my co-supervisor team, dr. Eef Theunissen and dr. Peter Stiers. Eef, your supervision during the master's internship set the stage for all of my PhD projects, and allowed me to hit the ground running. Throughout these last 4 years, it was truly a comfort knowing that your door was always open, and that I could come to you for any and all practical matters. Peter, although our time was more limited, the conversations we had opened my eyes up to just how much there is to know. I came away from our meetings immensely impressed by your depth and breadth of knowledge; I hope to be able to acquire even half as much during my academic career.

I would like to express my appreciation to the members of the assessment committee, prof. dr. Arjan Blokland, prof. dr. Draulio de Araujo, dr. Katrin Preller, prof. dr. Wim Riedel, and prof. dr. Therese van Amelsvoort, for accepting to read and evaluate my dissertation. Thank you 
dr. Marco Colizzi and dr. Kim van Oorsouw for also being a part of the promotion committee for the public defense. I am honored to be able to discuss my work with scientists whom I admire.

Needless to say, none of these projects would have been possible alone. All of the imaging studies were enabled by fMRI/MRS genius, Dr. Desmond Tse. Thank you, Des, for setting up (literally all of) the imaging sequences, and for being a mentor, reviewer-comment-battle-partner, and most importantly, a friend. Thank you to the Scannexus team, and particularly Esther Steijvers and dr. Chris Wiggins, for always picking up my manic scanner-troubleshooting calls, regardless of the time and day. Thank you dr. Cees van Leeuwen for your medical expertise, medical supervision, and for your chats. Annemie Jeukens, thank you for all your assistance throughout these last few years, and Anita van Oers, for your project help; because you both do everything so seamlessly, we often forget that there are many of us, but only one each of you. Last but certainly not least, thank you to Amanda Feilding and the Beckley Foundation, for helping to make possible some of the studies included in this dissertation. You've done truly great things for the field of psychedelic science, and I'm honored to play even such a small role in your mission.

The years spent during this PhD would not have been nearly as enjoyable (and so full of vlaai) without a department made up of such lovely colleagues. Our department days and Ardennes trips allowed a welcome break to relax and have a drink (or two $(\dot{)})$, while getting to know such an engaging group of people. Nadia, my time here at the department started with you, and thus I am very thankful for you for teaching me the ropes (and equally important, introducing me to the great Dieter Koblenz). Patrick, I am very grateful that you came and spent some time with us in Maastricht. I truly enjoyed our beers and chats, and had a fun time scaling the obstacle course into your Swiss heart. Eliza, when I first heard about you, I was told that you were someone whom "I had to meet" as we would get along very well and had such similar interests. Turns out this person was extremely right (Des, go figure), and you have evolved into a near and dear friend; one with whom I can always talk psychedelics with, and rely on for a good dance party. Malin, you are an inspiration in both modesty and perseverance. I'm continuously impressed on how you make things happen, while firmly standing your ground. I hope to continue our connection, both in the academic and personal sphere, and look forward to a time when we can reconnect in real life. Tom (and Gaby!), you two are the epitome of cool, and if any amount of that cool-ness rubbed off on me during our time spent together, I'm extremely thankful. If that is yet to be the case, I look forward to sharing future possibilities, in whichever city you guys end up in next. Pablo aka the new kid on the block, here's a forecasted thank you for our time together throughout the next few years. I would be a "yes" on a scale of 0 to 10 on excitedness-to-science with you. Last, but certainly not least, the biggest thank you, Johannes, for the many weekends you shared with me during the psilocybin study, and for the dark humor, hypnotic dance moves, and sincere friendship that you continue to share with me outside of it. Chookity. 
Frederick, Jessica, and Lilian, our cozy office and our time together has made it a pleasure to come to work. Just as one would experience at a trampoline park, we've shared both lows and highs throughout these last 4 years. From contemplating our decision to pick such a challenging life course (guys, we could've been gardeners), to lively interpretations of how loud the Dutch really are, and even the cultural experience that is a Belgian wedding, this has been cherished time with all of you. I hope that as we start to jump around to different stages of our lives and careers, we can continue to share both professional and personal experiences. And maybe, at the end of all of this, we can finally share the massive amount of alcohol we have accumulated in our office (and perhaps even a visit to an actual trampoline park, if you didn't get my hint).

As for my lovely paranymphs, if anyone is worthy of two mentions, it's the two of you. That said, I also found this particular section difficult to write. Again, not out of lack of things to say, but because of how special you two have become to me throughout these past 4 years; years in which we have shared so much! Calm writing retreats in Belgium, to late-night clubbing in Paris, with all the laughs and tears (and burritos!) in between. Jessica, your reliable-unpredictableness makes time spent around you a lot of fun. From hilarious outbursts, to impressive gin-drinking, and extremely appreciated listening skills, I'm beyond grateful to have had you as a beer-pong partner, NYE companion, and friend. Lili, over the years our friendship has blossomed into something so special that people even thought we were dating; and to be fair, I see their confusion. I've shared more experiences with you in the past 4 years than I have with any other friend. We've done all the fun stuff, exploring different countries (5, I believe), visiting festivals (2), sharing birthday parties (many), and even pizza in bed (1); and you've also always been there for me for the notso-fun stuff, checking in with me and lending an open, encouraging ear, as well as a voice of reason. Truth be told, I really could say loads of things about you, but they would all boil down to the fact of the matter: that you are a true friend, for whom I am extremely grateful for. Finally, and very importantly, throughout this time you both have continuously encouraged and helped me to find a balance between work and life; a previously unheard of concept, the understanding of which has helped me not only throughout the PhD, but also in day-to-day life. I hope to keep walking this balance with you both, and in return I will try my best to be around to "catch you outside", and to stop you from eating 2am buffet-hummus from the basement of smoke-filled clubs. Now, let's RELAX (!)

Neal, you are my oldest friend, and one of my biggest supporters. You've known many versions of myself over these past 11 (!) years, and you've loved and believed in all of them. Thank you for your stubborn loyalty, your never-ending encouragement, and above all, your friendship.

Finally, thank you to my family for their endless support. Grandma, Grandad, Nathan, and Darren, being on this side of the Atlantic has allowed me to catch up on precious time with you all. 
Chesterfield has become a much-loved second home during this time, and it has been truly lovely to share this home with you. Nathan, it has also been really special for me to be able to discuss my thesis work (and anything really) with a family member. Thank you for being that family member. Mom, Dad, Alex, Benjamin, and Grandma, when I said I was going to move 4000 miles away to try and study the therapeutic effects of strange, illegal drugs, you were behind me $100 \%$. And regardless of how many holidays we miss, or time we have to spend apart, you have only ever encouraged me to keep going and to follow my dreams, and have always supported me with your unwavering confidence. I miss you, I love you, and I thank you. 


\section{About the author}

Natasha Mason was born on March 1 ${ }^{\text {st }}, 1992$, in Valdosta, Georgia. She completed her high school education in Sparta, Wisconsin. In 2010, she enrolled in a Bachelor of Science program at the University of Wisconsin-Madison, with the intention of pursuing a degree in Pharmacy. During her studies, she became disillusioned with the current treatment options for psychiatric disorders, and became interested in investigating novel treatment approaches, like MDMA and psilocybin. She subsequently changed her major to a Bachelor of Science in Psychology, and gained experience working in various research labs with expertise in neuropsychopharmacology and psychiatry. Upon graduation in 2014, she moved to Maastricht, the Netherlands, with the aim to work with Dr. Kim Kuypers on her research assessing prosocial effects and mechanisms of psychedelic drugs.

In 2016 she graduated from Maastricht University's research master's program in Cognitive and Clinical Neuroscience (specialization, Neuropsychology), and began her PhD at the department of Neuropsychology and Psychopharmacology, under the supervision of Prof. Johannes Ramaekers and Dr. Kim Kuypers. During the PhD, she utilized multimodal study designs to assess drug-induced neuroadaptations and their influence on affect, behavior, and cognition. Within this focus, her strongest interest was, and continues to be, in the underlying mechanisms of psychedelic drug action; particularly those which may hold therapeutic value for increasingly common and difficult to treat disorders, like anxiety and depression. 


\section{Output}

\section{List of Publications}

\section{As part of this dissertation:}

Mason, N.L., Kuypers, K.P.C., Reckweg, J., Muller, F., Tse, D.H.Y., Da Rios, B., Toennes, S.W., Stiers, P., Feilding, A., Ramaekers, J.G. (2020) Brainwaves! Spontaneous and deliberate creative cognition during and after psilocybin exposure. In submission, Translational Psychiatry

Mason, N.L., Kuypers, K.P.C., Muller, F., Reckweg, J., Tse, D.H.Y., Toennes, S.W., Hutten, N.R.P.W., Jansen, J.F.A., Stiers, P., Feilding, A. \& Ramaekers, J.G. (2020) Me, myself, bye: regional alterations in glutamate and the experience of ego dissolution with psilocybin. Neuropsychopharmacology, DOI: 10.1038/s41386-020-0718-8.

Mason, NL., Mischler, E., Uthaug, M.V., Kuypers, K.P.C. (2019) Sub-acute effects of psilocybin on empathy, creative thinking, and subjective well-being. Journal of Psychoactive Drugs, DOI: 10.1080/02791072.2019.1580804.

Mason, NL, Theunissen EL, Hutten NRPW, Tse, D.H.Y., Toennes, S.W., Jansen, J.F.A., Stiers P., Ramaekers, J.G.. (2019) Reduced responsiveness of the reward system is associated with tolerance to cannabis impairment in chronic users. Addiction Biology, DOI: 10.1111/adb.12870.

Mason, NL., Theunissen EL., Hutten NRPW., Tse DHY., Toennes SW., Stiers P., Ramaekers, JG. (2018) Cannabis induced increase in striatal glutamate associated with loss of functional corticostriatal connectivity. European Neuropsychopharmacology. DOI: 10.1016/j.euroneuro.2018.12.003.

\section{Not as part of this dissertation:}

Mason, N.L. \& Kuypers, K.P.C. (2021) Acute and long-term effects of ayahuasca on higher-order cognitive processes in B.C. Labate \& C. Cavnar (Eds.), Ayahuasca healing and science.

Hutten, N.R.P.W. Mason, N.L., Dolder, P.C., Theunissen, E.L., Holze, F., Liechti, M.E., Feilding, A., Ramaekers, J.G., Kuypers, K.P.C. (2020). Mood and cognition after administration of low LSD doses in healthy volunteers: A placebo controlled dose-effect finding study. European Neuropsychopharmacology, DOI: 10.1016/j.euroneuro.2020.10.002. 
Holze, F., Liechti, M.E., Hutten, N.R.P.W., Mason, N.L., Dolder, P.C., Theunissen, E.L., Duthaler, U., Feilding, A., Ramaekers, J.G., Kuypers, K.P.C. (2020) Pharmacokinetics and pharmacodynamics of lysergic acid diethylamide microdoses in healthy participants. Clinical Pharmacology \& Therapeutics, DOI: 10.1002/cpt.2057.

Hutten, N.R.P.W., Mason, N.L., Dolder, P.C., Theunissen, E.L., Holze, F., Liechti, M.E., Varghese, N., Eckert, A., Feilding, A., Kuypers, K.P.C. (2020) Low doses of LSD acutely increase BDNF blood plasma levels in healthy volunteers. ACS Pharmacology \&Translational Science, DOI: 10.1021/acsptsci.0c00099.

Ramaekers, J.G., Hutten, N.R.P.W., Mason, N.L., Dolder, P.C., Theunissen, E.L., Holze, F., Liechti, M.E., Feilding, A., Kuypers, K.P.C. (2020) A low dose of lysergic acid diethylamide decreases pain perception in healthy volunteers. Psychopharmacology, DOI: 10.1177/0269881120940937.

Ramaekers, J.G., Mason, N.L., Theunnisen, E.L. (2020) Blunted highs: Pharmacodynmic and behavioral models of cannabis tolerance. European Neuropsychopharmacology, DOI: 10.1016/j.euroneuro.2020.01.006.

Mason, NL., Dolder, P.C., Kuypers, K.P.C. (2020) Reported effects of psychedelic use on those with low well-being given various emotional states and social contexts. Drug Science, Policy and Law, DOI:10.1007/s41465-019-00151-6.

Kettner, H., Mason, N. L., \& Kuypers, K. P. C. (2019). Motives for Classical and Novel Psychoactive Substances Use in Psychedelic Polydrug Users. Contemporary Drug Problems, 46, 304-320, DOI: 10.1177/0091450919863899.

Hutten, N.R.P.W., Mason, N.L., Dolder, P.C., Kuypers, K.P.C. Self-rated effectiveness of microdosing with psychedelics for mental and physical health problems among microdosers. Frontiers in Psychiatry. DOI: 10.3389/fpsyt.2019.00672.

Hutten NR, Mason NL, Dolder PC, Kuypers KP (2019) Motives and side-effects of microdosing with psychedelics among users. International Journal of Neuropsychopharmacology. Doi:10.1093/ijnp/pyz029.

Uthaug, M.V., Lancelotta, R., van Oorsouw, K., Kuypers, K.P.C., Mason, N.L., Rak, J., Sulakova, A., Jurok, R., Maryska, M., Kuchar, M., Palenicek, T., Riba, J., Ramaekers, J.G. (2019) A single inhalation of vapor from dried toad secretion containing 5-methoxy- $\mathrm{N}, \mathrm{N}$-dimethyltryptamine (5-MeO-DMT) in a naturalistic setting is related to sustained enhancement of satisfaction with life, mindfulnessrelated capacities, and a decrement of psychophathological symptoms. Psychopharmacology. DOI i: 10.1007/s00213-019-05236-w. 
Kuypers, K.P.C., De Sousa Fernandes Perna, E.B., Theunissen, E.L., Toennes, S.W., Mason, N.L., Hutten, N.R.P.W., Ramaekers, J.G. (2019) The novel psychoactive phenethylamine 4fluoroamphetamine produces a mild psychedelic state without affecting creative thinking. European Neuropsychopharmacology. DOI: 10.1016/j.euroneuro.2018.11.302.

Theunissen, E.L., Hutten, N.R.P.W., Mason, N.L., Toennes, S.W., Kuypers, K.P.C., Ramaekers, J.G. (2019) Neurocognition and subjective experience following acute doses of the synthetic cannabinoid JWH-018: Responders versus nonresponders. Cannabis and Cannabinoid Research. DOI: 10.1089/can.2018.0047.

Kuypers, K.P.C., De Sousa Fernandes Perna, E.B., Theunissen, E.L., Toennes, S.W., Mason, N.L., Hutten, N.R.P.W., Ramaekers, J.G. (2019) A first-in-man study with 4-fluoroamphetamine demonstrates it produces a mild psychedelic state. Journal of Psychoactive Drugs. DOI: 10.1080/02791072.2019.1569286.

Dolder, P.C., de Sousa Fernandes Perna, E.B., Mason, N.L., Hutten, N.R.P.W., Toennes, S.W., Theunissen, E.L., Ramaekers, J.G., Kuypers, K.P.C. (2018) Independent elevation of peripheral oxytocin concentrations and reduction in cognitive empathy during 4-fluoroamphetamine intoxication. Human Psychopharmacology, DOI: 10.1002/hup.2680.

Uthaug, M.V., van Oorsouw, K., Kuypers, K.P.C., van Boxtel, M., Broers, N.J., Mason, N.L., Toennes, S.W., Riba, J., Ramaekers, J.G. (2018) Sub-acute and long-term effects of Ayahuasca on affect and cognitive thinking style and their association with ego dissolution. Psychopharmacology, DOI:10.1007/s00213-018-4988-3.

De Sousa Fernandes Perna, E.B., Theunissen, E.L., Dolder, P.C., Mason, N.L., Hutten, N.R.P.W., Toennes, S.W., Kuypers, K.P.C, Ramaekers, J.G. (2018) Safety profile and neurocognitive function following 4-fluoroamphetamine (4-FA) administration in humans. Frontiers in Pharmacology, 9(713), DOI: 10.3389/fphar.2018.00713.

Kuypers, K.P.C., de Sousa Fernandes Perna, E.B., Dolder, P.C., Toennes, S.W., Theunissen, E.L., Mason, N.L., Hutten, N.R.P.W., Ramaekers, J.G. (2018) Drug liking and wanting, not impulsive action or reflection is increased by 4-fluoroamphetamine. Psychopharmacology, 18-28. doi: 10.1007/s00213-018-4931-7

Mason, N.L. \& Kuypers, K.P.C. (2018) Mental health of a self-selected sample of psychedelic users and self-medication practices with psychedelics. Journal of Psychedelic Studies, 2(1), 45-52. DOI:10.1556/2054.2018.006.

Theunissen, E. L., Hutten, N. R., Mason, N. L., Toennes, S. W., Kuypers, K.P.C., De Sousa Fernandes Perna, E.B., \& Ramaekers, J. G. (2017). Neurocognition and subjective experience following acute 
doses of the synthetic cannabinoid JWH-018: A phase 1, placebo-controlled, pilot study. British Journal of Pharmacology,175(1), 18-28. DOI:10.1111/bph.14066.

\section{Presentations}

\section{Conference Presentations}

Acute effects of psilocybin on glutamate concentration levels, functional connectivity, and subjective state. (2020) Talk presented at: Interdisciplinary Conference on Psychedelic Research virtual conference

Acute effects of psilocybin on glutamate concentration levels, functional connectivity, and subjective state. (2020) Talk presented at: $33^{\text {rd }}$ European College of Neuropsychoopharmacology virtual conference

Acute effects of psilocybin on glutamate concentration levels, functional connectivity, and subjective state. (2020) Poster presented at: $33^{\text {rd }}$ European College of Neuropsychoopharmacology virtual conference

Acute effects of psilocybin on glutamate concentration levels, functional connectivity, and subjective state. (2019) Talk presented at: International Society of Psychedelic Research conference, New Orleans, USA.

(Sub-)Acute effects of psilocybin on creativity, empathy, and satisfaction with life. (2019) Talk presented at: Breaking Convention, London, UK.

Acute effects of psilocybin on glutamate and subjective state: a placebo controlled, experimental study. (2019) Talk presented at: Dutch Neuroscience Conference

Subacute effects of psilocybin on creativity, empathy, and subjective well-being. (2019) Poster presented at: Dutch Neuroscience Conference,

The Good, the Bad, and the Intoxicated. (2019) Talk presented at: Pint of Science Festival, Maastricht, the Netherlands

Subacute effects of psilocybin on creativity, empathy, and subjective well-being. (2019) Poster presented at: VI International conference on novel psychoactive substances, Maastricht, the Netherlands

Brain Kinetics of Neurometabolites during THC Intoxication. (2018) Talk presented at: International Cannabinoid Research Society Sympsoium, Leiden, the Netherlands. 
Psilocybin and Cognitive Flexibility: A placebo controlled experimental study. (2018) Talk presented at: Beyond Psychedelics Conference, Prague, Czech Republic

Brain Kinetics of Neurometabolites during THC Intoxication. (2017) Talk presented at EURON PhD Days, Maastricht, Netherlands.

Brain Kinetics of Neurometabolites during THC Intoxication. (2017) Talk presented at EURON Workshop: Psychopharmacology from Laboratory to Clinic, Crete, Greece.

Brain Kinetics of Neurometabolites during THC Intoxication. (2017) Poster presented at EURON Workshop: Psychopharmacology from Laboratory to Clinic, Crete, Greece.

Brain Kinetics of Neurometabolites during THC Intoxication. (2017) Poster presented at: Drug Science 2017 conference, Berlin, Germany.

Brain Kinetics of Neurometabolites during THC Intoxication. (2017) Talk presented at Faculty of Psychology and Neuroscience Research Day 2017, Maastricht, NL

\section{Invited Presentations}

Me, Myself, Bye: Regional alterations in glutamate and the experience of ego dissolution with psilocybin. (2020). Talk presented to the Center for Psychedelic and Consciousness Research, John Hopkins, USA.

Psychedelics and Cognition. (2019) Talk presented at: Psychedelic Science Collective, Rotterdam, the Netherlands

Brain Kinetics of Neurometabolites during THC Intoxication. (2019) Talk presented at: Neuropsychology \& Psychopharmacology Research Day 2019; Maastricht, NL.

Psilocybin as a tool to enhance creativity. (2018) Talk presented at: The Innbetween, Maastricht, the Netherlands

Mind-bending, heart-mending. (2018) Talk presented at: TEDx PhD night, Maastricht, the Netherlands

LSD Microdosing- A Dose Finding Study. (2018) Talk presented at: Open the Doors Symposium, Amsterdam, the Netherlands 
Psilocybin and Cognitive Flexibility: Therapeutic Implications. (2018) Talk presented at: Amsterdam Psychedelic Research Association Symposium, Amsterdam, the Netherlands

Psilocybin and Cognitive Flexibility: Therapeutic Implications. (2018) Talk presented at: Psykedeelinakymia 2018, Helsinki, Finland

Psilocybin and Cognitive Flexibility: A translational study. (2018) Talk presented at: Faculty of Psychology and Neuroscience Research Day, Maastricht, Netherlands.

\section{Workshops Organized}

The good, the bad, and the intoxicated. (2020) Pint of Science, Maastricht, the Netherlands.

The acute and long-term effects of psychedelics on cognitive flexibility and well-being. (2018) Beyond Psychedelics, Prague, Czech Republic.

\section{Grants and Honors}

Selected early career speaker for New Findings Symposium at $33^{\text {rd }}$ ECNP congress 2020

Top 10 most-downloaded articles by Taylor \& Frances in 2019, and most read article from the Journal of Psychoactive Drugs as of 2020, Sub-acute effects of psilocybin on empathy, creative thinking, and subjective well-being

Top 10\% most downloaded papers 2018-2019, Reduced responsiveness of the reward system is associated with tolerance to cannabis impairment in chronic users.

Best Poster Award, Drug Science 2017, Brain Kinetics of Neurometabolites during THC Intoxication Awarded funding from The Beckley Foundation, for project: Psilocybin as a tool for enhanced divergent thinking and positive learning mechanisms, 2017 
